\title{
Scanning X-Ray Nano-Diffraction on Eukaryotic Cells:
}

\section{From Freeze-Dried to Living Cells}

\author{
Dissertation \\ for the award of the degree \\ "Doctor rerum naturalium" \\ of the Georg-August-Universität Göttingen \\ within the doctoral program \\ Göttingen Graduate School for Neurosciences, Biophysics, and \\ Molecular Biosciences (GGNB) \\ of the Georg-August University School of Science (GAUSS) \\ submitted by

\section{Britta Weinhausen} \\ from Seesen
}

Göttingen 2013 


\section{Thesis Committee:}

Prof. Dr. Sarah Köster

Institute for X-Ray Physics

Georg-August-Universität Göttingen

Prof. Dr. Tim Salditt

Institute for X-Ray Physics

Georg-August-Universität Göttingen

Prof. Dr. Helmut Grubmüller

Department for Theoretical and Computational Biophysics

Max-Planck-Institute for Biophysical Chemistry, Göttingen 


\section{Members of the Examination Board:}

Reviewer: Prof. Dr. Sarah Köster

Institute for X-Ray Physics

Georg-August-Universität Göttingen

Second Reviewer: Prof. Dr. Tim Salditt

Institute for X-Ray Physics

Georg-August-Universität Göttingen

\section{Further members of the Examination Board:}

Prof. Dr. Helmut Grubmüller

Department for Theoretical and Computational Biophysics

Max-Planck-Institute for Biophysical Chemistry, Göttingen

Dr. Florian Rehfeldt

Third Institute of Physics - Biophysics

Georg-August-Universität Göttingen

Prof. Dr. Simone Techert

Research Group Structural Dynamics of (Bio)chemical Systems

Max-Planck-Institute for Biophysical Chemistry, Göttingen

Deutsche Elektronen-Synchrotron, Hamburg

Institute for X-Ray Physics

Georg-August-Universität Göttingen

Prof. Dr. Andreas Janshoff

Institute for Physical Chemistry

Georg-August-Universität Göttingen

\section{Date of oral examination:}

05.12 .2013 



\section{Contents}

$\begin{array}{ll}\text { 1. Introduction } & 1\end{array}$

2. Higher-Order Organization of Cytoskeletal Biopolymers 5

2.1. The Cytoskeleton . . . . . . . . . . . . . . . . 5

2.2. Keratin Intermediate Filaments ． . . . . . . . . . . . . . . . . . 6

2.2.1. Assembly into Filaments, Bundles and Networks . . . . . . . . 7

2.2.2. Specific Higher-Order Organizations . . . . . . . . . . . . . . . 10

2.2.3. Hard Keratins . . . . . . . . . . . . . . . . . . . . . . 12

2.3. Non-Keratin Higher-Order Cytoskeletal Structures in Biological Cells 12

3. Scanning X-Ray Nano-Diffraction 15

3.1. Scanning Diffraction with Nano-Beams . . . . . . . . . . . . . . . . . 15

3.1.1. Contrast Mechanisms for Scanning Imaging . . . . . . . . . . 16

3.1.2. Diffraction at the Nano-Scale . . . . . . . . . . . . . . . . . . 18

3.1.3. Radiation Damage and Dose . . . . . . . . . . . . . . . . . . . 19

3.2. Synchrotron Set-ups . . . . . . . . . . . . . . . . . . 21

3.2.1. ID13 Beamline, ESRF . . . . . . . . . . . . . . . . . . 22

3.2.2. P10 Beamline, PETRA III . . . . . . . . . . . . . . . . 23

3.2.3. cSAXS Beamline, SLS . . . . . . . . . . . . . 24

3.2.4. Comparison of the Different Set-Ups . . . . . . . . . . . . 26

4. Biological System and Sample Preparation 29

4.1. Cell Line and Culture . . . . . . . . . . . . . . . . . . . . . . . . . . . 29

4.2. Preparation of Cellular Samples for Synchrotron Experiments . . . . 30

4.2.1. Growing Cells on Silicon Nitride Membrane Windows . . . . . 30

$4.2 .2 . \quad$ Living Cells . . . . . . . . . . . . . . . . . . . . 32

4.2.3. Fixed-Hydrated Cells . . . . . . . . . . . . . . . . . . 32

4.2.4. $\quad$ Freeze-Dried Cells . . . . . . . . . . . . . . . . . . . . . . . . . 32

4.2.5. Cellular IF Preparation . . . . . . . . . . . . . . . . . . . 36

4.3. Sample Environments for Hydrated Cells . . . . . . . . . . . . . . . . 41

4.3.1. Sandwich Wet Chambers . . . . . . . . . . . . . . . . . . . 42 
4.3.2. Fabrication of Microfluidic Devices . . . . . . . . . . . . . . . 43

4.3.2.1. Photolithography . . . . . . . . . . . . . . 43

4.3.2.2. Kapton-NOA 81 Device with one $\mathrm{Si}_{3} \mathrm{~N}_{4}$ Membrane Window ................... . 44

4.3.2.3. Final Device Assembly . . . . . . . . . . . . . . . 46

5. Scanning Nano-Diffraction on Freeze-Dried Cells 49

5.1. Experiment . . . . . . . . . . . . . . . . . . 49

5.2. Results from Measurements at ID13 Beamline . . . . . . . . . . . . . 52

5.2.1. Cells with a Keratin Network . . . . . . . . . . . . . . . . 52

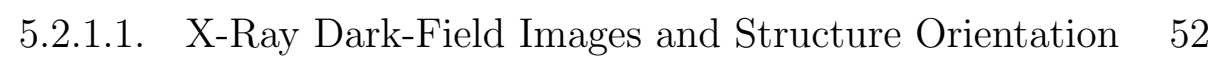

5.2.1.2. Radial Intensities of Averaged and Single Scattering Patterns . . . . . . . . . . . . . 58

5.2.2. Cells without a Keratin Network . . . . . . . . . . . . 63

5.2.2.1. X-Ray Dark-Field Images and Structure Orientation 63

5.2.2.2. Radial Intensities of Averaged Scattering Patterns . 65

5.2.3. Cells treated with the IF Lysis Buffer . . . . . . . . . . . . . 66

5.2.3.1. X-Ray Dark-Field Images and Structure Orientation 66

5.2.3.2. Radial Intensities of Averaged Scattering Patterns . 68

5.3. Results from Measurements at the P10 Beamline . . . . . . . . . . . 70

5.3.1. X-Ray Dark-Field Images and Structure Orientation . . . . . 70

5.3.2. Radial Intensities of Averaged Scattering Patterns . . . . . . . 72

5.3.2.1. Comparison of Power Law Exponents . . . . . . . . . 76

5.3.3. Radial Intensities of Single Scattering Patterns . . . . . . . . . 77

5.4. Results from Measurements at the cSAXS Beamline . . . . . . . . . . 82

5.4.1. X-Ray Dark-Field Images and Structure Orientation . . . . . 82

5.4.2. Radial Intensities of Averaged and Single Scattering Patterns 84

5.5. Summary and Closing Discussion . . . . . . . . . . . . . . . . 88

6. Scanning Nano-Diffraction on Fixed-Hydrated Cells 95

6.1. Experiment . . . . . . . . . . . . . . . . 95

6.2. Results from Measurements at the cSAXS Beamline . . . . . . . . . . 97

6.2.1. Images Employing Different X-ray Contrasts . . . . . . . . . . 97

6.2.2. Radial Intensity of Averaged Scattering Patterns . . . . . . . 101

6.3. Results from Measurements at the ID13 Beamline . . . . . . . . . . . 103

6.3.1. X-Ray Dark-Field Images . . . . . . . . . . . . . . . . . . . . 103

6.3.2. Radial Intensity of Averaged Scattering Patterns . . . . . . . 105

6.4. Results from Measurements at the P10 Beamline . . . . . . . . . . . 108

6.4.1. X-Ray Dark-Field Images . . . . . . . . . . . . . . . . . . 108 
6.4.2. Radial Intensity of Averaged Scattering Patterns . . . . . . . 110 6.4.2.1. Comparison of Power Law Exponents. . . . . . . . . 112 6.4 .2 .2 . Influence of Fitting Range . . . . . . . . . . . . . . . 114

6.4.2.3. Influence of Beam Attenuation . . . . . . . . . . . . 116

6.4.3. Radial Intensity of Single Scattering Patterns . . . . . . . . . 117

6.5. Specific Challenges . . . . . . . . . . . . . . . . . 120

6.5.1. Radiation Damage . . . . . . . . . . . . . . . . 120

6.5.2. Heat Input . . . . . . . . . . . . . . . . . . . 123

6.5.3. Buffer Degassing . . . . . . . . . . . . . . . . . 127

6.6. Summary and Closing Discussion . . . . . . . . . . . . . . . 128

7. Scanning Nano-Diffraction on Living Cells 133

7.1. Experiment . . . . . . . . . . . . . . . . . . . . . 133

7.2. Results from Measurements at the ID13 Beamline . . . . . . . . . . . 135

7.3. Results from Measurements at the P10 Beamline . . . . . . . . . . . 137

7.3.1. X-Ray Dark-Field Images . . . . . . . . . . . . . . . . . 137

7.3.2. Radial Intensities of Averaged Scattering Patterns . . . . . . . 138

7.4. Specific Challenges . . . . . . . . . . . . . . . . . . . . . . . 142

7.4.1. Storage and Culture of Living Cells . . . . . . . . . . . . . . . 142

7.4.2. Imaging of Living Cells . . . . . . . . . . . . . . . . . . . . 142

7.4.3. Radiation Damage . . . . . . . . . . . . . . . . . 143

7.5. Summary and Closing Discussion . . . . . . . . . . . . . . . . 144

\begin{tabular}{ll}
\hline 8. Comparison of the Different Preparation Methods & 147
\end{tabular}

8.1. Power Law Exponents . . . . . . . . . . . . . . . . . . . . . . 147

8.2. Average Radial Intensity Profiles . . . . . . . . . . . . . . . . . . . . 148

8.3. Summary and Closing Discussion . . . . . . . . . . . . . . . . . . 150

\begin{tabular}{ll}
\hline 9. Summary and Conclusion & 153
\end{tabular}

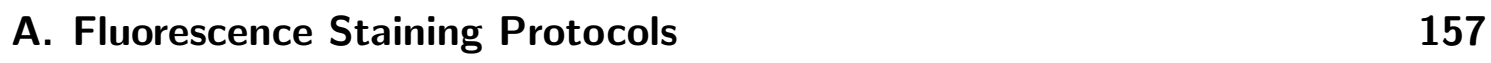

A.1. Protocol for Staining of Filamentous Actin and DNA . . . . . . . . . 157

A.2. Protocol for Staining of Microtubules and DNA . . . . . . . . . . 158

\begin{tabular}{ll}
\hline B. Supplementary Data on Freeze-Dried Cells & 159
\end{tabular}

B.1. Results from Measurements at the P10 Beamline . . . . . . . . . . . 159

C. Supplementary Data on Fixed-Hydrated Cells 165

C.1. Results from Measurements at the ID13 Beamline . . . . . . . . . . . 165

C.2. Results from Measurements at the P10 Beamline . . . . . . . . . . 167 
\begin{tabular}{|l}
\hline D. Supplementary Data on Living Cells \\
181
\end{tabular}

D.1. Results from Measurements at the P10 Beamline . . . . . . . . . . . 181

\begin{tabular}{lr}
\hline E. Ptychography Measurements & 185
\end{tabular}

E.1. Beamtime in May 2012 . . . . . . . . . . . . . . . . . . . . . . . . 185

E.1.1. Siemens Star Reconstruction . . . . . . . . . . . . . . . . 185

E.1.2. Cell Reconstruction . . . . . . . . . . . . . . . . . 186

E.2. Beamtime in February 2013 . . . . . . . . . . . . . . . . . . . 188

\begin{tabular}{ll}
\hline List of Abbreviations and Symbols & 192
\end{tabular}

\begin{tabular}{|ll}
\hline List of Figures & 201
\end{tabular}

\begin{tabular}{ll}
\hline List of Tables & 204
\end{tabular}

\begin{tabular}{|rr}
\hline Bibliography & 217
\end{tabular}

\begin{tabular}{lr}
\hline Publications & 219
\end{tabular}

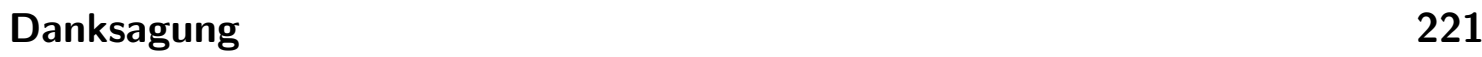

\begin{tabular}{ll}
\hline Lebenslauf & 225
\end{tabular} 


\section{Introduction}

Biological cells are the basic unit of live. Despite their importance and ubiquity, a complete understanding of their hierarchical organization and their function at the molecular level is still lacking due to their exceptional complexity. An important component of eukaryotic cells is the cytoskeleton, which is a complex and dynamic network of different biopolymers and plays an important role in, e.g., the determination of cellular shape, mechanical properties, and consequently specific cellular functions $[1-3]$. Cytoskeletal protein networks are mainly composed of three different classes of proteins: actin filaments, microtubules and intermediate filaments. These proteins can further form higher-order structures like bundles or paracrystalline arrays of filaments [2, 4]. One example for the higher-order organization of cytoskeletal proteins are bundles and networks of keratin intermediate filaments (KIFs). KIFs occur mainly in epithelial cells and provide these cells with the necessary mechanical properties to withstand external stress [1]. Mutations in genes encoding for keratin proteins can result in skin diseases, which show an intolerance of the skin against external forces and the formation of blisters as a consequence of light touches [5 7]. Detailed knowledge about the filament arrangement in keratin bundles in cells is still lacking. The small structure sizes in the order of tens of nanometers require high resolution imaging techniques, which are compatible with soft matter samples and non-invasive sample preparations. The determination of a general bundle structure is further impeded by structural variations of keratin bundles between different tissues and, at least in terms of the diameter, also within one cell.

X-rays provide an ideal probe for studying structures at the nano-scale and are routinely employed for investigating the structure and the composition of biological systems, making use of the variety of different techniques like X-ray fluorescence analysis [8], coherent diffractive imaging (CDI) as well as ptychography $9 \sqrt{12}$ or scanning small-angle X-ray scattering (SAXS) 13 17]. The small wavelength in principle allows for structure determination with atomic precision and comparatively thick samples can be investigated non-invasively due to the high penetration depth of hard X-rays. By raster scanning the sample with a small beam, structural information obtained from individual scattering patterns in reciprocal space can be combined with positional information in real space 13 17]. For micrometer sized 
beams, this method has been applied to a variety of different samples including biological materials like bone, tooth, muscles or wood [13 20]. A further reduction of the probed sample volume is achieved by employing focused beams with spot sized well below $100 \mathrm{~nm}[21-24]$, which allows for studying the local structure of highly heterogeneous or small specimens.

A particularly interesting and challenging object of research are biological cells, which exhibit a complex and highly heterogeneous architecture on length scales ranging from nanometers to micrometers. Most structural studies on single biological cells employ various visible light microscopy or electron microscopy techniques, but the possibility of X-ray imaging or X-ray fluorescence analysis of single cells has been demonstrated as well [8 12, 25, 27]. In most X-ray studies of cellular samples, the specimens were investigated in a dried form, which has the advantage of increasing the electron density contrast between sample and surrounding medium, thereby leading to a stronger scattering signal, as well as a comparatively easy sample handling. However, the natural environment for most biological systems is aqueous and consequently measurements should ideally be performed on fully hydrated samples [28], since the structure is very likely to change upon dehydration. For the structure determination of biological molecules, this approach is followed by combining results obtained from crystallography, which exhibit a high resolution down to the atomic level, with SAXS measurements in solution 29 31].

Measurements on hydrated cellular samples require specific sample environments. Since the electron density contrast is lower for hydrated samples, high photon flux synchrotron beamlines are needed to obtain a good signal-to-noise ratio. Therefore the sample environment and window material need to be chosen deliberately to be resistant to the radiation as well as to avoid a strong background signal, and suited for cell culture. An excellent growth substrate as well as window material is provided by silicon nitride membrane windows [32]. Imaging of fixed-hydrated and (initially) living eukaryotic cells using scanning transmission X-ray microscopy and spectromicroscopy have been demonstrated in the soft X-ray regime [33]. Cellular organelles could be identified and near edge X-ray absorption fine structure (NEXAFS) spectra were recorded, yielding different calcium concentrations for different cellular regions. Recently, CDI measurements on hydrated yeast cells were presented [28]. In both studies, sealed wet chambers built of two silicon nitride membrane windows were used. Sealed wet chambers, however, do not allow for manipulating the cells during the measurements. Microfluidic devices, by contrast, provide an adaptable and welldefined sample environment allowing for sample manipulation by, e.g., changing the $\mathrm{pH}$ or the concentration of chemicals. A variety of microfluidic devices based on different materials and fabrication techniques has been proposed in the past to meet 
the requirements of X-ray experiments [34 38]. The specific demands of the studied objects impose further limitations on the device design and the material choice. Living cells are probably among the most challenging sample types: Well-defined growth conditions like temperature or $\mathrm{pH}$ value must be combined with a continuous nutrient supply and, in the case of adherent cells, a specific substrate must be provided.

The goal of this thesis was to apply scanning X-ray diffraction using a nano-focused beam to samples of biological cells in order to probe the structure of cytoskeletal bundles and networks of keratin intermediate filaments. As a model system the cell line SK8/18-2 was employed, which expresses fluorescently labeled keratins that assemble in these cells into complex networks. Cellular samples were prepared using different methods, starting from well-established freeze-dried samples and going on to fixed-hydrated and finally living cells. Using this approach, potentially invasive and structure altering steps during the sample preparation could be reduced or completely avoided, which allows for probing the native sample structure. However, the requirements of the different sample types on the sample environment and the sample handling during the experiments are more complex for the hydrated and particularly living cells as compared to the freeze-dried cells. In this context, the development of X-ray compatible microfluidic devices allowing for measurements on living cellular samples was an important aspect.

This thesis is organized as follows: Chapter 2 presents an introduction to the biological objects under study and points out several examples for the occurrence of higher-order organization of cytoskeletal proteins in biological cells. The used method and the employed set-ups for the measurements are described in chapter 3. In chapter 4, the preparation of cellular samples using different methods and the fabrication of X-ray compatible sample environments for hydrated samples are described. The results from measurements on freeze-dried, fixed-hydrated and living cells are presented in chapter 5, chapter 6, and chapter 7, respectively. A comparison of the results obtained for samples prepared by the different methods is given in chapter 8 . Finally, chapter 9 closes with a summary of the most important results and a conclusion.

Parts of this thesis have been published in New Journal of Physics [39] and Lab on a Chip [40] or have been prepared for publication [41]. 



\section{Higher-Order Organization of Cytoskeletal Biopolymers}

This chapter gives a short introduction to the biological system under study, i.e. keratin intermediate filaments, which are one component of the cytoskeleton in eukaryotic cells. First, a general conception of the cytoskeleton with emphasis on intermediate filaments is presented and afterwards the description is focused on keratin intermediate filaments. Finally, examples for the higher-order organization of cytoskeletal proteins are briefly presented. In all these examples, the specific mechanical and structural properties of the resulting filament arrangement are important for the biological system to function correctly.

\subsection{The Cytoskeleton}

The cytoskeleton of eukaryotic cells is a complex and dynamic network of different biopolymers, which serves as a cellular scaffolding and thereby determines the cellular shape, the mechanical properties, and the spatial organization inside the cell. It furthermore enables the generation of forces inside the cell or between the cell and its environment. [1-3]. In combination, all of these aspects determine the cell type specific functions. The main constituents of the cytoskeleton are three different classes of proteins, which are microfilaments (MFs, also called actin filaments), microtubules (MTs) and intermediate filaments (IFs), in combination with various cross-linking proteins. Networks of these filaments penetrate the entire cytoplasm (compare Fig. 2.1).

MFs and MTs, on the one hand, are built of the globular proteins actin and tubulin, respectively, which polymerize into polar and highly dynamic filaments with a diameter of about $8 \mathrm{~nm}(\mathrm{MFs})$ and $25 \mathrm{~nm}$ (MTs) [2]. Due to the filament polarity, these filaments can serve as tracks for motor proteins, allowing for a directed movement. On the other hand, IFs assemble from rod-like proteins, which form non-polar filaments with a diameter of about $10 \mathrm{~nm}[2,42]$. Furthermore, while actin and tubulin are highly conserved and occur in humans only in three major sub-types, IFs comprise a large family of different proteins. In humans, for example, IFs are encoded 


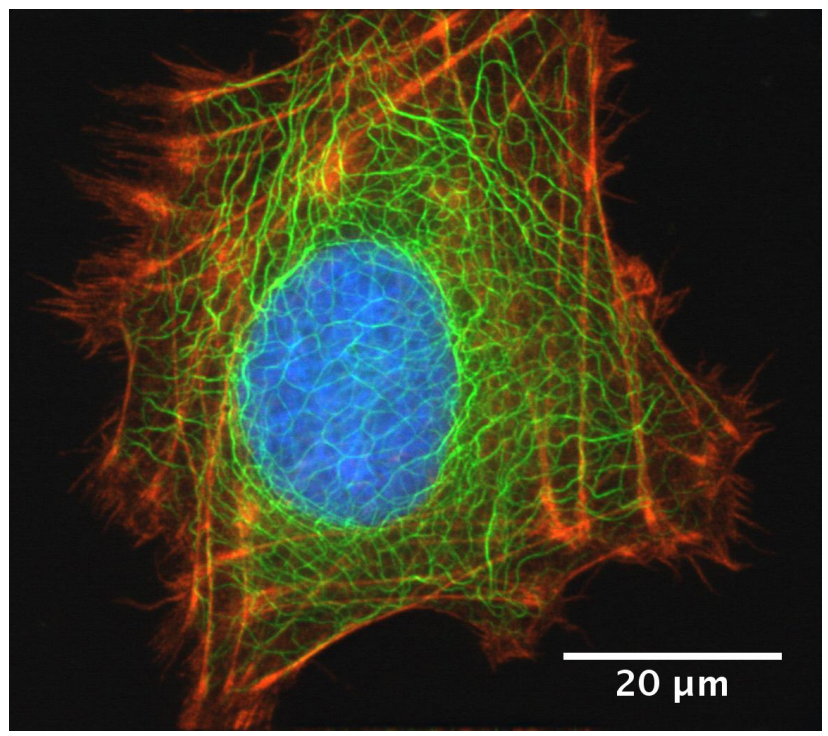

Fig. 2.1.: Fluorescence microscopy image of a cell, where the keratin intermediate filament network is represented in green, the actin filaments in red and the DNA in the nucleus in blue. [Courtesy of Jannick Langfahl-Klabes.]

by (at least) 70 genes [42]. On basis of the amino-acid-sequence identity, IF proteins are commonly grouped into six different types 42,44 . Tab. 2.1 gives an overview of the different IF types along with their tissue distribution. Despite their differences in the primary amino acid sequence, all IF proteins share a common structural organization, which is based on an about $45 \mathrm{~nm}$-long $\alpha$-helical rod domain and the non- $\alpha$-helical amino-terminal head and carboxy-terminal tail domains [42, 45].

\subsection{Keratin Intermediate Filaments}

Keratin intermediate filaments (KIFs) are the most diverse type among IFs and constitute the type I and type II IF proteins. Keratins can further be subdivided into cytokeratins, which are expressed in various epithelial cells, and so-called "hard" keratins (see section 2.2.3), which are used to build, e.g. hair, nail or feather [46]. A major function of cytokeratins is to provide epithelial cells with the necessary mechanical properties to withstand external impacts like pressure or shear forces [1, 47]. Mutations in genes encoding for keratin proteins can result in skin diseases such as Epidermolysis bullosa simplex showing an intolerance of the skin against external forces and the formation of blisters as a consequence of light touches [5 7]. Epithelial cells can be divided into simple (single-layered) and complex (multilayered) types [48]. The expression of different keratins as well as the total keratin content highly varies with the specific cell type. For instance, simple epithelia usually express K8 and K18, which makes up less $5 \%$ of the total protein content in these 


\begin{tabular}{lll}
\hline type & IF protein & tissue/cell distribution \\
\hline I & acidic keratins & epithelial cells (also in hair, nail, etc.) \\
II & neutral-basic keratins & epithelial cells (also in hair, nail, etc.) \\
III & desmin & muscle \\
& vimentin & mesenchymal cells \\
& glial fibrillary acidic protein & astrocytes \\
& peripherin & peripheral neurons \\
IV & neurofilaments & central nervous system neurons \\
& nestin & stem cells, neuroepithelia \\
& $\alpha$-internexin & central nervous system neurons \\
& synemin & muscle \\
V & lamins & all nuclei \\
VI & filensin & lens fiber cells \\
& phakinin & lens fiber cells \\
\hline
\end{tabular}

Tab. 2.1.: Overview of the six different IF types and their tissue distribution 42,44 .

cells 49,50. By contrast, in cultured keratinocytes, which are the predominant cell type in the epidermis, about $30 \%$ of the total protein consists of keratin [49,50]. Keratinocytes further change their appearance in the different layers of the epidermis [2]. Furthermore, in the stratum corneum, i.e. the outermost layer of the epidermis, even $85 \%$ of the total protein are represented by keratin [49,50]. A detailed summary of the expression pattern and the function of KIFs in different types of epithelial cells is presented in reference [51].

\subsubsection{Assembly into Filaments, Bundles and Networks}

IFs can be categorized into three distinct assembly groups based on their assembly mechanisms: keratins (assembly group I), vimentin-like proteins (assembly group II) and lamins (assembly group III) [52]. In this context, the characteristic feature of keratins is the formation of heterodimers that are always built of an acidic and neutral-basic keratin (a type I and a type II IF protein). In the IF dimers, the $\alpha$ helical rod domains form a coiled-coil structure. Recently, the crystal structure of the central coiled-coil domain of a K5/K14 dimer has been solved using X-ray crystallography [53].

During in vitro filament assembly, heterodimeric tetramers assemble laterally into so-called unit-length filaments (ULFs) with a length of 50-60 nm and four tetramers per cross-section, which then anneal longitudinally into filaments with a diameter 


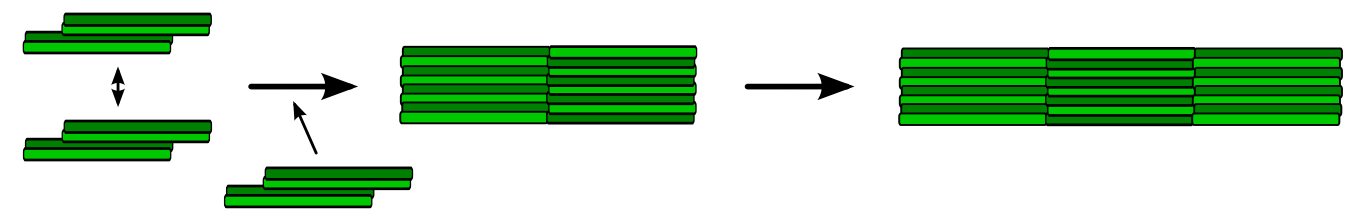

Fig. 2.2.: Schematic model of the KIF assembly. Heterodimeric tetramers assemble laterally into so-called unit-length filaments and almost simultaneously ULFs anneal longitudinally into long filaments. [Figure based on [52.]

of about $10 \mathrm{~nm}$ 46, 52,54. A schematic of the assembly process is depicted in Fig. 2.2 . In contrast to the vimentin-like assembly mechanism, the longitudinal annealing of keratin ULFs is much faster and occurs almost simultaneously with the ULF formation [46]. Only for low protein concentrations, ULFs are present [54]. Furthermore, a radial compaction step, as suggested for vimentin, is not observed for keratins [54]. On a higher structural level, KIFs assemble into bundles of filaments and into highly cross-linked networks. In vitro, bundling and network formation of KIFs can be triggered by raising the ionic strength of the buffer solution, for instance by adding of $10 \mathrm{mM}$ sodium chloride to the assembly buffer [55 57] or by the addition of the IF associated protein filaggrin [58,59].

Mainly the simple epithelial keratins K8/K18 and the epidermal keratins K5/K14 have been employed in the in vitro studies summarized above. However, the assembly of KIFs in vitro has been also applied for studying the effect of mutations in the amino acid sequence or unconventional pairing (like K5/K18 dimers) on the different steps of filament assembly or bundle formation as well as on the mechanical properties of the resulting networks $[48,55,56,60]$. Interestingly, filament and bundle formation have been also observed for unconventional pairs like K8/K14 and $\mathrm{K} 5 / \mathrm{K} 18$, but these systems displayed different mechanical properties from the natural pair K8/K18 and K5/K14 as probed by rheology [48]. Furthermore, three specific region of the K14 molecule, i.e. one region at the tail and two at the rod, have been identified, which determine the bundling properties of K5/K14 dimer [56]. The prevalent model for KIF assembly in vivo is based on fluorescence microscopy studies on cultured epithelial cells that were transfected with genes encoding for fluorescently labeled keratins [61 67]. A detailed review of the proposed assembly and disassembly cycle of keratins has been recently given by Windoffer et al. 67]. A schematic of the keratin cycle describing the process filament and bundle formation, is presented in Fig. 2.3. KIF precursors form in the cell periphery in close vicinity to the focal adhesion sites from soluble keratin oligomers (nucleation). The KIF precursors continuously elongate by end-to-end annealing (elongation), which is inline with the proposed assembly mechanism from in vitro studies [46,52,54, and are 


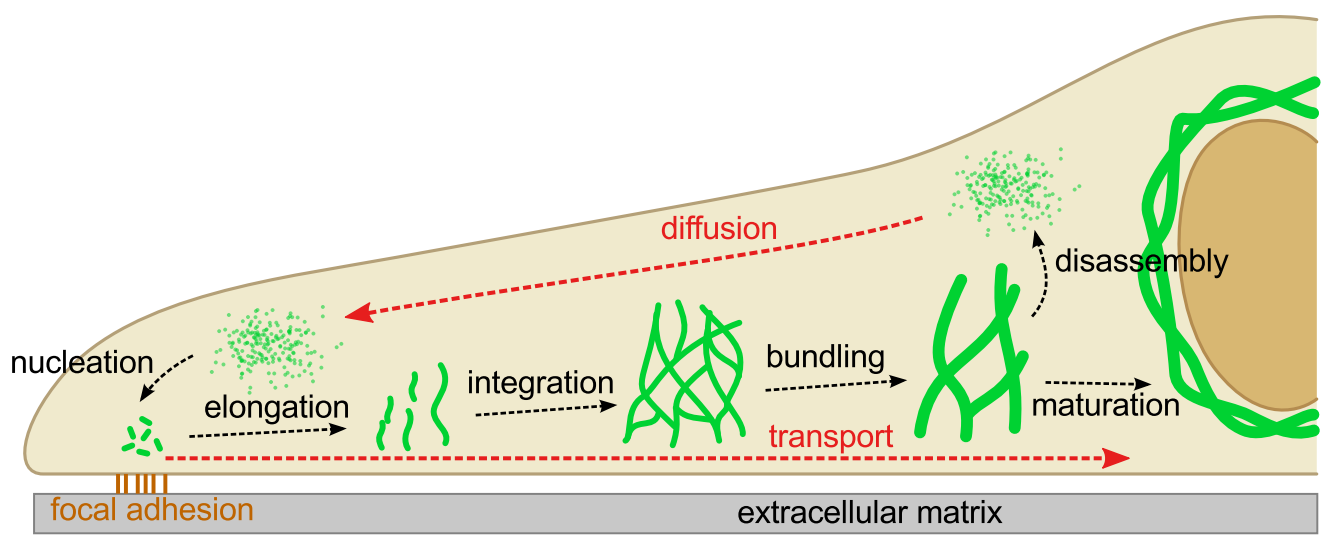

Fig. 2.3.: Schematic of the keratin cycle. KIF precursors form in the cell periphery from soluble keratin oligomers (nucleation). The KIF precursors continuously elongate by endto-end annealing (elongation) and move towards the nucleus in a process that depends on actin (transport). The elongated KIF particles integrate end-on into the existing KIF network (integration) and translocate further towards the nucleus (transport). Bundling of filaments increases towards the nucleus by lateral association of filaments and bundles (bundling). The inward-moving KIF bundles can either dissociate into soluble oligomers (disassembly) that diffuse through the cytoplasm (diffusion) and are reused again in the cell periphery for filament assembly, or the KIF bundles are stabilized (maturation) and form, e.g., a stable cage-like structure around the nucleus. [Figure based on 67].]

transported towards the nucleus along actin stress fibers (transport). Whether the KIF precursors are identical to the ULFs found in vitro could not be assigned so far, because the resolution of standard light microscopes is not high enough to allow for the identification of single ULFs [67]. The elongated KIF particles integrate end-on into the existing KIF network without disruption of its continuity (integration) and translocate further towards the nucleus (transport). Bundling of filaments increases towards the nucleus by lateral association of filaments and bundles (bundling), as can be also observed from the apparent bundle thicknesses in the fluorescence microscopy image in Fig. 2.1. Upon bundling the mechanical stability increases and the turnover reduces. The inward-moving KIF bundles can either dissociate into soluble oligomers (disassembly) that diffuse through the cytoplasm (diffusion) and are reused again in the cell periphery for filament assembly, or the KIF bundles are stabilized (maturation) and form, e.g., a stable cage-like structure around the nucleus. Bundling is controlled by different factors, which are IF associated proteins, intrinsic and isotype-specific properties of the KIFs and phosphorylation (compare [58, 67, and references therein). 


\subsubsection{Specific Higher-Order Organizations}

The keratin content, the expression pattern of KIFs, and consequently also the specific structural organization the KIFs in the cytoplasm, highly depend on the certain cell type. Furthermore, the detailed structural organization of the individual KIFs considerably determines the mechanical properties of the cells. Therefore, a few specific examples for the keratin arrangement in the cytoplasm of different epithelial cell types are presented in this section for illustration.

A confocal fluorescence microscopy image of the keratin network in a SK8/18-2 cell (compare section 4.1) is displayed in Fig. 2.4. This cell line was generated by transfection of IF-free SW-13 cells with fluorescently labeled keratins K8/K18, which organize in a network of filaments and bundles as expected for (simple) epithelial cells 62, 63, 68. Furthermore, scanning electron microscopy (SEM) images of the purified KIF network in a SK8/18-2 cell are presented in Fig. 2.4b-d (compare reference 69,70 ] for details concerning the cell preparation). Towards the nucleus, the bundling and the thickness of the keratin bundles increases up to diameters of about $150 \mathrm{~nm}$. This kind of network morphology was also described above in the

(a)
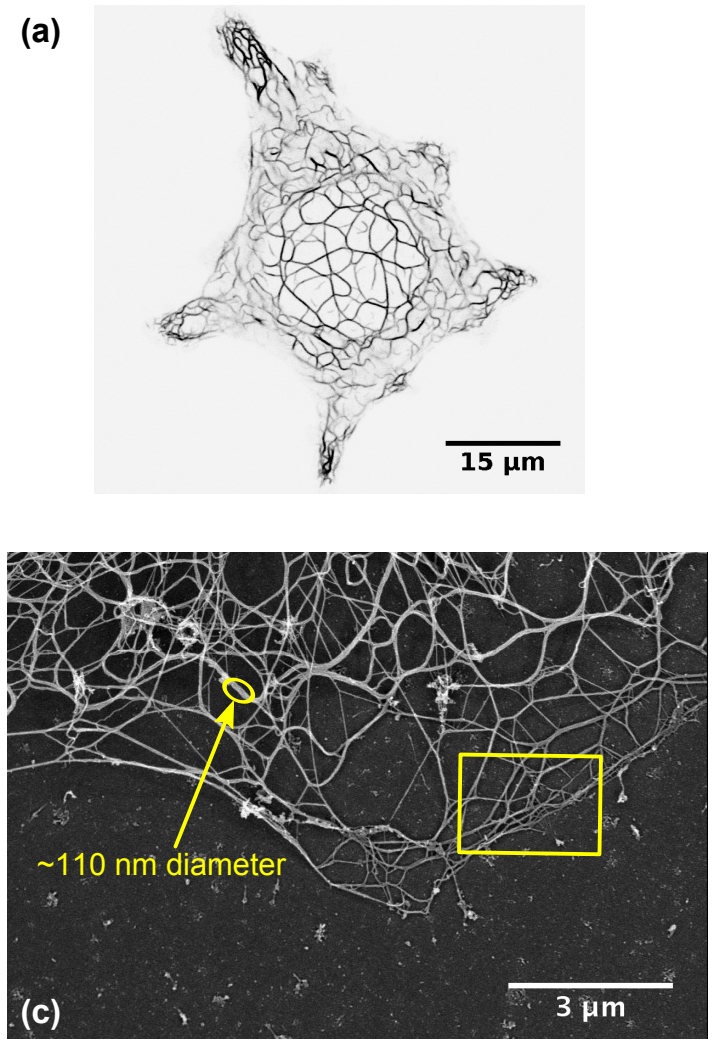
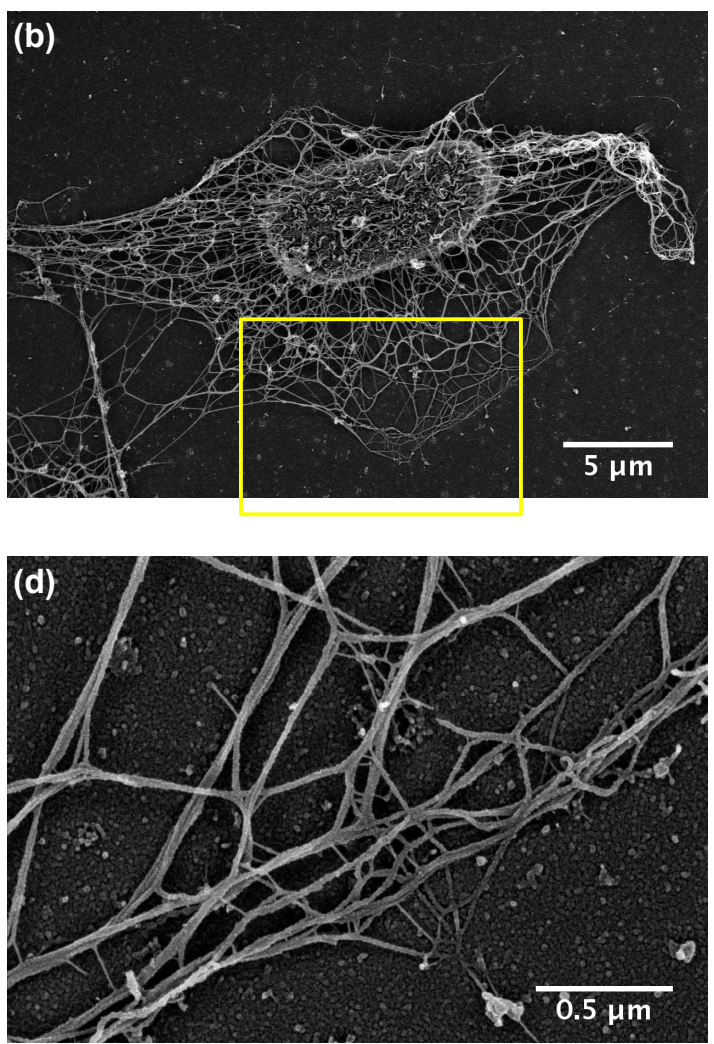

Fig. 2.4.: (a) Inverted confocal fluorescence microscopy image and (b-d) SEM image of the keratin network in a SK8/18-2 cell at different magnifications. [(a) Courtesy of Jens Nolting. (b-d) Courtesy of Prof. Dr. P. Walter and Prof. Dr. M. Beil, University of Ulm.] 


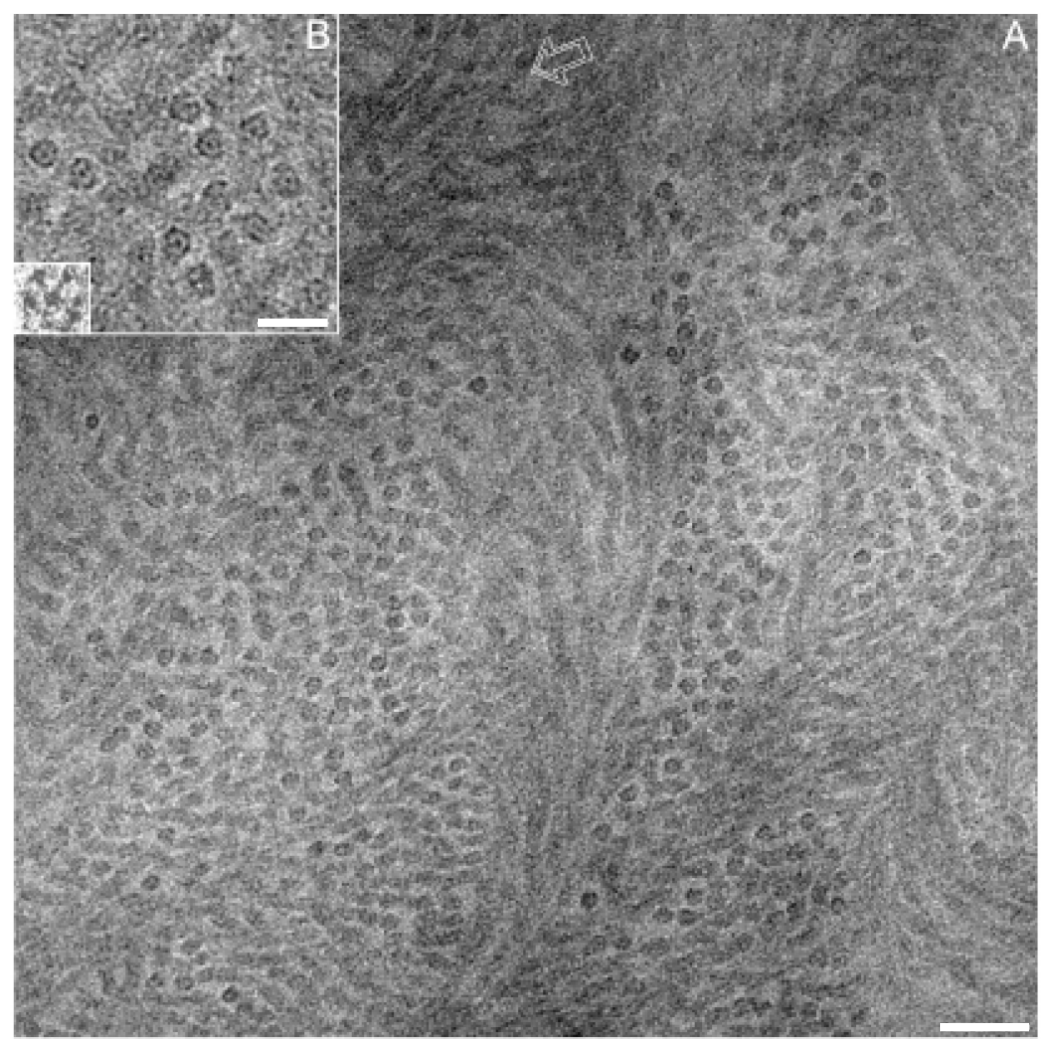

Fig. 2.5.: Cryo transmission electron micrograph of a vitreous section of the stratum corneum. Individual KIFs appear as groups of dark (electron dense) dots. The inset in (b) shows an individual filament. The scale bars are $50 \mathrm{~nm}$ in (a) and $10 \mathrm{~nm}$ in (b). [Figure adapted from 75.$]$

context of the keratin cycle. Similar network morphologies have been observed for various epithelial cell lines $[56,62,71,74]$. However, the detailed arrangement of the individual filaments in these KIF bundles is not known and might depend on the cell type and on the location in the cell.

In the epidermis, the keratin content in the cells increases towards the outer layers and in the stratum corneum up to $85 \%$ of the total protein mass are keratins [49 51]. Therefore, particularly in the stratum corneum, the individual KIFs are densely packed within the cells. A cryo transmission electron micrograph of a vitreous section of the stratum corneum is displayed in Fig. 2.5 [75. The individual KIFs appear as groups of dark, i.e. electron dense, dots in the electron micrograph with a diameter of about $7.8 \mathrm{~nm}$ and an average filament center-to-center distance of about $16 \mathrm{~nm} \mathrm{[75]}$. To describe the higher-order organization of the KIFs in these cells, Norlén and co-workers suggested a cubic rod packing of the filaments (compare [75, 76] for further details on the model). In the viable cell layers of the epidermis the higher-order organization of the KIFs shows distinct differences 75, 76. Here, the average filament center-to-center distance was about $11 \mathrm{~nm}$ and a hexagonal 
packing of the individual KIFs has been suggested 75,76. However, several aspects of these models, for instance how the transition between the two distinct structural organizations proceeds, are not fully understood.

\subsubsection{Hard Keratins}

A special group of keratin intermediate filaments are the so-called "hard" keratins, which are the building blocks for, e.g. hair, nail, scales or feather [46, 77]. Hard keratins contain typically more sulfur than the cytokeratins encountered in (soft) tissue, which allows for stronger cross-linking of individual filaments via disulfide bridges [77. Filaments of hard keratins form densely packed arrays with an hexagonal arrangement of the individual filaments. These structures of hard keratins have been extensively studied using X-ray diffraction, dating back to first studies in the 1930s. Four different types of diffraction patterns were found, representing different molecular structures: the $\alpha$-pattern from hard mammalian keratinized tissues, the feather-pattern from hard avian or reptilian tissue, the $\beta$-pattern from stretched mammalian keratin and the amorphous pattern from, for example, the cuticle of animal hair [77]. In this context, the coiled-coil structure of the keratin filamentsubunits (heterodimers) was first predicted for mammalian keratins [78].

\subsection{Non-Keratin Higher-Order Cytoskeletal Structures in Biological Cells}

The formation of bundles of filamentous proteins is not restricted to keratin intermediate filaments, but occurs also for other cytoskeletal proteins. In this section, examples for the higher-order arrangement of cytoskeletal proteins other than KIFs are presented.

A bundle of cross-linked actin filaments forms the core of stereocilia of hair cells [4], which are located in the inner ear and enable hearing and the sense of balance. An SEM image of the stereocilia bundle of a hair cell is displayed in Fig. 2.6a. Transmission electron microscopy images of thin sections through stereocilia either in bundle direction or perpendicular, revealed highly ordered array of actin filaments as shown schematically in Fig. 2.6b [4]. Due to the mechanical properties of the actin filament bundles, the stereocilia behave like rigid rods that, upon mechanical stimuli, deflect without bending elastically along their shafts. This property of stereocilia is important for mechanoelectrical transduction, i.e. the conversion of a mechanical stimulus into an electric signal that can be carried by neurons to the central nervous system. During this process, ion channels, which are triggered by a tip linker that 

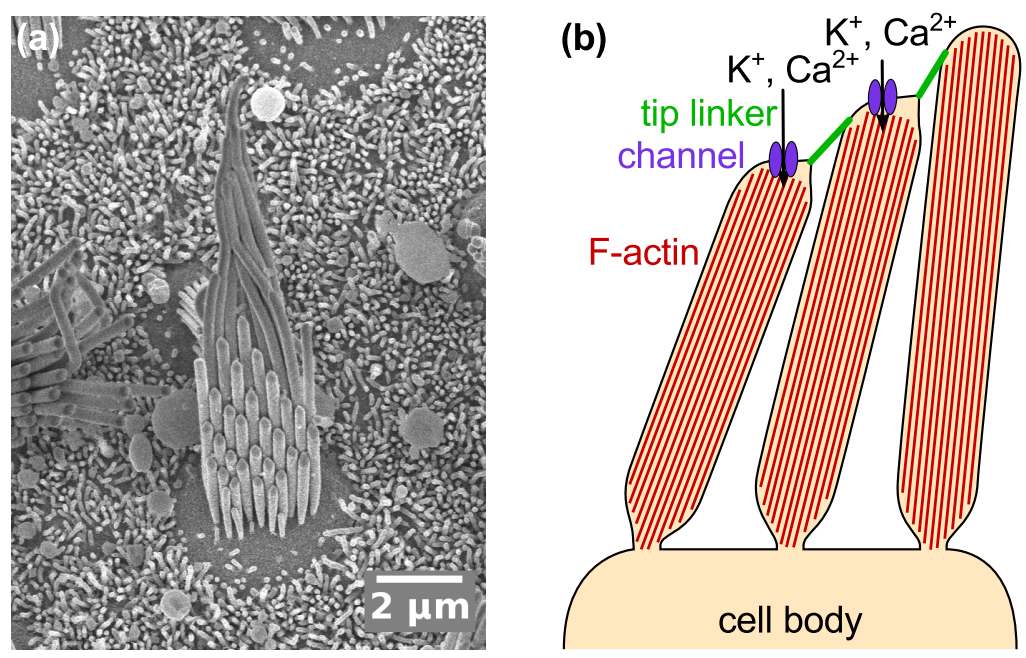

Fig. 2.6.: Actin filament bundle in hair cells. (a) SEM image of the stereocilia bundle of a hair cell. (b) Schematic of the actin filament organization in stereocilia and the effect of stereocilia deflection. Upon deflection of stereocilia calcium ions can permeate through the mechanotransduction channel. [(a) Courtesy of Dr. Valeria Piazza. (b) Simplified figure based on [79].]

connects different stereocilia, open upon deflection, thereby allowing for an influx of calcium and potassium ions into the stereocilia [80]. The ion influx then leads to an electrical signal, which can be further transferred to and processed by neurons. Similar bundles of actin filaments are present in microvilli [81]. Microvilli are small extensions of the plasma membrane that occur for instance on the surface of absorptive epithelial cells of the intestine and highly enlarge the absorptive area of these cells [2]. A different type of actin filament bundles are so-called stress fibers [2,82]. Stress fibers are mainly constituted of actin filaments and myosin II motor proteins, which allows them to contract actively. In cells, stress fibers are involved, e.g., in cell migration or in the response to mechanical stresses [82].

Another example are neurofilaments, which belong to the type IV IF proteins and are found in high concentrations in the axons of vertebrate neurons [2, 83]. Here, neurofilaments create, together with microtubules and actin filaments, a cross-linked network along the length of the axons [84]. Neurofilaments are important for the growth of the axon and for the precise maintenance of the axon diameter, which is related to the conducting velocity. Transmission electron micrographs of thinsection perpendicular to the axon revealed an ordered pattern of neurofilaments, which is altered upon chemical modification to the neurofilament proteins [84, 85]. Since the mechanical properties are inevitably linked to the detailed structural arrangement in vivo, it is important to precisely understand these structural organizations on a local scale. 



\section{Scanning X-Ray Nano-Diffraction}

This chapter gives a short introduction to the employed method: scanning X-ray diffraction using a nano-focused beam. The different possibilities as well as challenges of imaging and diffraction with nanometer-sized beams are presented and discussed. Basic knowledge of the properties of X-rays as well as of the interaction of X-rays with matter and the diffraction of X-rays by ordered structures are presupposed and can be found for instance in references [86 88]. Furthermore, lengthy derivations of formulas that are described in detail in the literature are not repeated here, but it is referred to the original literature at the respective positions.

\subsection{Scanning Diffraction with Nano-Beams}

X-rays provide an ideal probe for studying structures at the nano-scale. The small wavelength in principle allows for structure determination with atomic precision, which is well achieved for X-ray diffraction from crystalline structures, and the high penetration depth of hard X-rays allows for the investigation of comparatively thick samples without sectioning. An important property of X-ray diffraction is the fact that the recorded diffraction pattern represents an ensemble average of the probed structure in the sample. For crystalline material, averaging over a large ensemble has the advantage of enhancing the recorded diffracted intensity and leading to a sharper line profile of the individual reflections (Laue function). However, for inhomogeneous systems, information about the local structure is lost upon averaging over a large ensemble and therefore spatially resolved X-ray diffraction is highly desired.

The advent of micrometer-sized X-ray beams opened the possibility of probing the local sample structure or composition in small volumes in the order of the beam size and recording maps of structural parameters by scanning the sample. Scanning with a micrometer-sized beam in combination with small-angle X-ray scattering (SAXS) has been applied to a variety of different samples including biological materials such as bone, tooth, muscle or wood [13 19]. Furthermore, real space images of the sample with a spatial resolution in the order of the beam size can be generated by employing different contrast mechanisms as know from scanning transmission X-ray microscopy $89 \sqrt{93}$. A short summary of the principles behind the mechanisms of 
absorption contrast, differential phase contrast and dark-field contrast are presented in section 3.1.1.

The continuous improvement of X-ray optics further made it possible to achieve focal spot sizes below $100 \mathrm{~nm}[21-24]$. The step toward the use of a sub-micron beam further reduced the probed sample volumes to dimensions, which allow to investigate structural properties of nano-scale objects without averaging over a large ensemble [20]. In section 3.1.2, important aspects of X-ray diffraction with nanometer sized beams are presented.

\subsubsection{Contrast Mechanisms for Scanning Imaging}

In the limit of geometrical optics, the wave field after passing through an object with a refractive index $n(\mathbf{r})=1-\delta(\mathbf{r})+i \beta(\mathbf{r})$ can be described by the projection approximation 88]. Here, $\delta$ and $\beta$ are real numbers, which are for X-rays much less than unity, e.g. $\delta=7.2 \times 10^{-6}$ and $\beta=1.2 \times 10^{-8}$ for carbon at $8 \mathrm{keV}$. A schematic of the assumed geometry is presented in Fig. 3.1. Let $\psi(x, y, z=0)$ be the wave field at $z=0$ before the object. Then the wave field after the object can be approximated by

$$
\psi\left(x, y, z=z_{0}\right) \approx \psi(x, y, z=0) \exp \left(i k z_{0}\right) \exp \left[-i k \int_{0}^{z_{0}}[\delta(\mathbf{r})-i \beta(\mathbf{r})] \mathrm{d} z\right],
$$

with the absolute value of the wave vector $k=2 \pi / \lambda$. In this so-called projection approximation, all changes to the wave field due to interaction with the object are accumulated along streamlines of the incoming beam. Therefore, the integrated real part $\delta$ of the index of reflection leads to a phase shift of the outgoing wave, and the integrated imaginary part $i \beta$ is responsible for the absorption in the object. For a detailed derivation of Eq. 3.1, the reader is referred to reference [88], particularly section 2.2 .

The absorption (contrast) of an object can then be determined by taking the squared modulus of Eq. 3.1 and identifying $|\psi(\mathbf{r})|^{2}$ with the intensity $I(\mathbf{r})$ :

$$
I\left(x, y, z=z_{0}\right)=\exp \left[-2 k \int_{0}^{z_{0}} \beta(\mathbf{r}) \mathrm{d} z\right] I(x, y, z=0) .
$$

For a homogeneous material with a projected thickness $T(x, y)$, Eq. 3.2 resembles the Beer-Lambert law of absorption, i.e.

$$
I\left(x, y, z=z_{0}\right)=\exp [-\mu T(x, y)] I(x, y, z=0),
$$

with the linear attenuation coefficient $\mu=2 k \beta$.

The accumulated phase shift of the wave as it passes through the object is

$$
\phi(x, y)=-k \int_{0}^{z_{0}} \delta(\mathbf{r}) \mathrm{d} z .
$$


A lateral gradient in the phase shift leads to a (local) lateral deflection of the beam, which is given by

$$
\alpha_{x}=\frac{\lambda}{2 \pi} \frac{\partial \phi(x, y)}{\partial x}, \alpha_{y}=\frac{\lambda}{2 \pi} \frac{\partial \phi(x, y)}{\partial y}
$$

with the deflection angles $\alpha_{x}$ and $\alpha_{y}$ in $x$ - and $y$-direction, respectively [89. This effect can be exploited in scanning techniques to generate differential phase contrast images of the sample, by determination of the deflection of the beam on the detector for each position in a scanning measurement.

Eqs. 3.5 can be derived using the sketch in Fig. 3.2. Here, an X-ray beam passes in vertical direction through an inhomogeneous object with a refractive index $n(\mathbf{r})=1-\delta(\mathbf{r})$, i.e. without absorption. The accumulated phase shift then depends on the lateral position and therefore an incoming wave is refracted, as indicated by the red lines denoting two planes with constant phase. $\mathbf{n}$ denotes the unit vector in propagation direction at the second plane of constant phase. The phase shifts $\phi\left(x=x_{0}, y\right)$ and $\phi\left(x=x_{0}+\Delta x, y\right)$ at the positions $x_{0}$ and $x_{0}+\Delta x$ are given by Eq. 3.4. The spatial difference $L_{1}$ and $L_{2}$ between the two planes of equal phase can be related to the phase shift as

$$
\begin{aligned}
L_{1} & =\frac{\lambda}{2 \pi}\left(\phi\left(x_{0}, y\right)+m\right) \\
L_{2} & =\frac{\lambda}{2 \pi}\left(\phi\left(x_{0}+\Delta x, y\right)+m\right),
\end{aligned}
$$

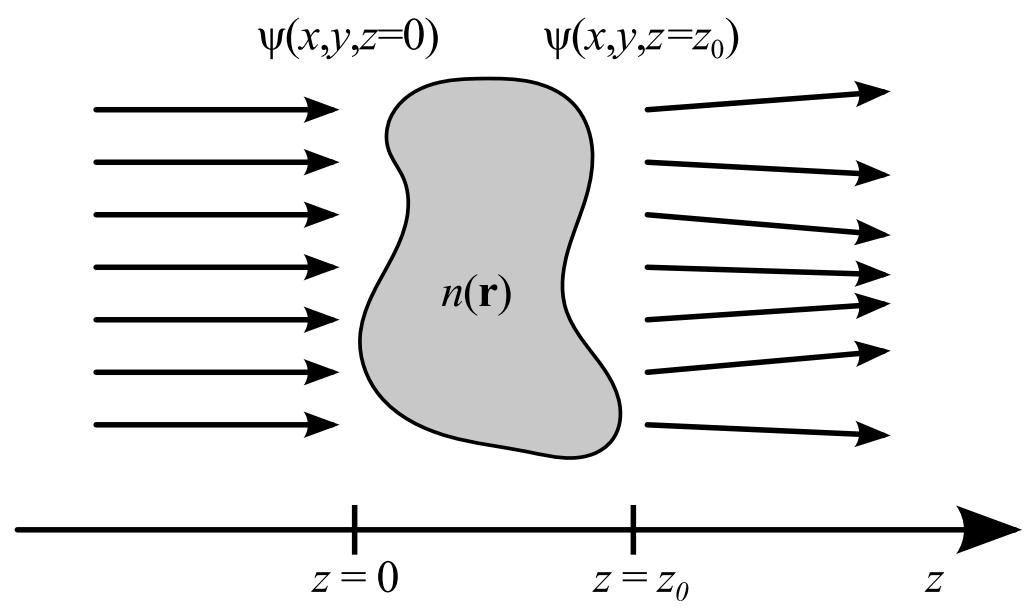

Fig. 3.1.: Schematic of the geometry assumed for the derivation of the projection approximation. The incident wave field $\psi$ passes through an object with a refractive index $n(\mathbf{r})$, which leads to the absorption of the beam as well as the accumulation of a phase shift, which causes local variations of the direction of propagation. 


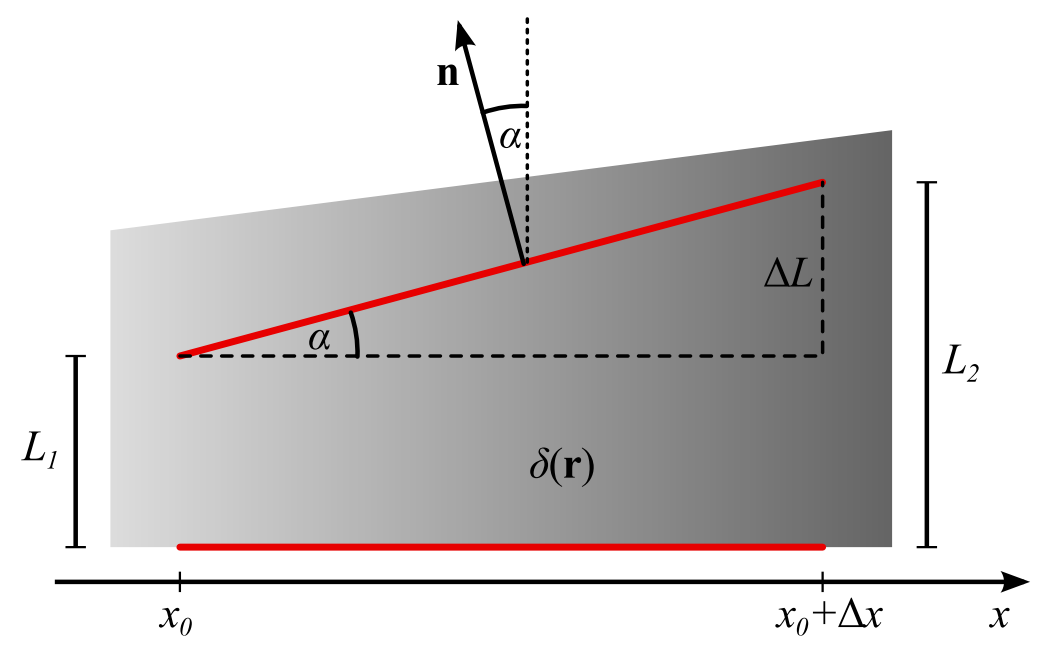

Fig. 3.2.: Relationship between the deflection angle $\alpha$ and the real part of the refractive index $\delta$. An X-ray beam passes in vertical direction through an inhomogeneous object. The red lines indicated two planes with constant phase and $\mathbf{n}$ denotes the unit vector in propagation direction at the second plane of constant phase. [Figure based on [86].]

with $\lambda$ being the wavelength in vacuum and $m$ a real number. The deflection angle $\alpha$ can then be calculated:

$$
\alpha \approx \frac{L_{2}-L_{1}}{\Delta x}=\frac{\lambda}{2 \pi} \frac{\phi\left(x_{0}+\Delta x, y\right)-\phi\left(x_{0}, y\right)}{\Delta x} \stackrel{\Delta x \rightarrow 0}{\longrightarrow} \frac{\lambda}{2 \pi}\left(\frac{\partial \phi(x, y)}{\partial x}\right)_{x_{0}} .
$$

Therefore the deflection angle is proportional to the gradient of the phase shift, which the object imposes on the wave field.

X-ray dark-field imaging is another mode of image formation. Here, similar to the case of visible light dark-field microscopy, the undiffracted light is blocked and only the scattered intensity is recorded on the detector [92,94]. When using a twodimensional pixel detector, it is further possible to generate X-ray dark-field images by masking the primary beam after recording the diffraction patterns or to select only specific regions of the reciprocal space, which correspond to scattering from distinct structures in the sample.

\subsubsection{Diffraction at the Nano-Scale}

The electron density distribution $\rho(\mathbf{r})$ of an object in real space is related to the measured scattered intensity in reciprocal space by a Fourier transform [86, 87]:

$$
I(\mathbf{q}) \propto\left|\int \rho(\mathbf{r}) e^{-i \mathbf{q} \cdot \mathbf{r}} \mathrm{d} \mathbf{r}\right|^{2}=|\mathcal{F}[\rho(\mathbf{r})]|^{2} .
$$

Here, $\mathbf{q}=\mathbf{k}_{f}-\mathbf{k}_{i}$ is the scattering vector, which is defined by the wave vectors $\mathbf{k}_{i}$ and $\mathbf{k}_{f}$ of the incoming and the scattered wave, respectively, and $\mathcal{F}$ denotes the 
Fourier transform. Further, a plane wave is assumed for the illumination. In case of illuminating the sample with a nanometer sized beam, not all scatterers are excited with the same field strength and therefore, the probe wave field $P$ needs to be taken into account. The recorded intensity on the detector is then given by 95,96

$$
I(\mathbf{q}) \propto|\mathcal{F}[P(\mathbf{r}) \cdot \rho(\mathbf{r})]|^{2} .
$$

Using the convolution theorem, the product of the electron density $\rho$ of the sample and the complex wave field $P$ in the sample plane is equivalent to a convolution of the Fourier transforms $\mathcal{F}[\rho]$ and $\mathcal{F}[P]$ in the detector plane:

$$
I(\mathbf{q}) \propto|\mathcal{F}[P(\mathbf{r}) \cdot \rho(\mathbf{r})]|^{2}=|\mathcal{F}[P(\mathbf{r})] * \mathcal{F}[\rho(\mathbf{r})]|^{2} .
$$

Therefore, employing a small beam in a diffraction experiment leads to a blurring of the recorded scattering patterns. The extent of blurring is stronger for smaller beam sizes and depends on the specific properties of the incoming wave field like the dimension and the phase.

Several practical aspects need to be considered in a diffraction experiment using a nanometer sized X-ray beam. On the one hand, diffraction using a small beam with a diameter in the order of the size of the object under study has the advantage of reducing the background signal due to scattering from the surrounding material compared to the signal from the specimen, which has been exploited for micrometer sized crystals and X-ray micro-beams in the past. On the other hand, the scattered intensity increases with the number of illuminated unit cells of a crystal and therefore employing smaller beam leads to a decrease of the total scattering signal. Furthermore, it is only possible to probe periodicities or correlations in the sample on length scales that are smaller than the probed volume. In particular for crystalline structures, the dimensions of the unit cells must be well below the beam size to probe the lattice arrangement.

\subsubsection{Radiation Damage and Dose}

Radiation damage to a (biological) sample due to ionizing radiation, like X-rays, is a severe issue in diffraction experiments. In macromolecular crystallography the radiation damage process is commonly subdivided in primary and secondary damage $97-99]$. Primary damage refers to the ionization of an atom due to photoelectric absorption or Compton scattering. Secondary damage is attributed to the energy dissipation process of the photo electron, during which highly reactive species such as radicals, hydrated protons or hydrated electrons are created. Whereas at cryogenic temperatures below $110 \mathrm{~K}$ nearly all radicals are immobilized, all products 
can diffuse through the specimen and contribute to the secondary damage at room temperatures [99]. For the events of primary damage it can be further differentiated between direct damage, i.e. ionization of the protein, and indirect damage, i.e. ionization of the surrounding solvent [99]. Furthermore it is important to distinguish between global and specific damage [99]. In macromolecular crystallography, global damage can be monitored by, e.g., a loss of the measured reflection intensity at high resolution corresponding to increasing disorder in the sample. Specific structural damage refers to the breakage of covalent bonds in a reproducible order, i.e. first disulfide bonds break, then glutamates and aspartates are decarboxylated, followed by specific structural modifications to other amino acids [99, 100]. These specific processes occur well before the global damage can be observed and might therefore lead to artifacts in the reconstructed electron density distribution 101.

From the discussion above it is obvious that the extent of primary radiation damage depends on the number of absorbed photons. Since further the deposited energy in the sample is the relevant parameter, it is useful to describe radiation damage in terms of the dose $D$ with the unit $\mathrm{Gy}=\mathrm{J} / \mathrm{kg}$ [101. The maximum tolerable dose $D_{\text {tol }}$ to obtain a given resolution $\Delta r$ between $0.1 \mathrm{~nm}$ and $10 \mathrm{~nm}$ at cryogenic conditions is from experiments on macromolecular crystals empirically found to be 102

$$
D_{\mathrm{tol}}[\mathrm{Gy}] \approx 10^{8} \times \Delta r[\mathrm{~nm}] .
$$

Hence, the tolerable dose increases approximately linearly with the requested resolution $\Delta r$.

The applied dose in an experiment can be estimated using the photon flux at the specific set-up. For an incident photon fluence $F_{0}$ per unit area integrated over the measurement time, the transmission through the sample is determined by BeerLambert's law, i.e. $F(d)=F_{0} \exp (-\mu d)$ with the absorption coefficient $\mu$ and the penetration depth $d$. The number of absorbed photons at the sample surface is then given by $[\partial F / \partial d]_{d=0}=F_{0} \mu$, which results in a deposited energy at the surface of $\mu F_{0} h \nu$ with the photon energy $h \nu[102]$. Therefore, the surface dose is given by

$$
D=\frac{\mu F_{0} h \nu}{\rho}
$$

with the mass density $\rho[102]$. In the following chapters, the average surface dose per step of a raster scan is approximated by

$$
D=\frac{\mu N_{0} h \nu}{\rho \Delta_{x} \Delta_{y}}
$$

with the number of photons $N_{0}$ incident on the sample in one exposure and the lateral step sizes $\Delta_{x}$ and $\Delta_{y}$. Note that the dose is averaged over the step sizes and 
not over the beam size for this estimation. Furthermore, for the dose calculation it is assumed that the cellular material can be described by the empirical average formula $\mathrm{H}_{50} \mathrm{C}_{30} \mathrm{~N}_{9} \mathrm{O}_{10} \mathrm{~S}$ and a mass density $\rho=1.35 \mathrm{~g} / \mathrm{cm}^{-3} 102$.

The extent of secondary damage depends on different factors such as the solvent, the temperature and the presence or absence of radical scavengers 99, 103. In hydrated cellular samples, radicals and in particular the hydroxyl radicals are considered to be the major damage producers 104 106. It is assumed that more than $80 \%$ of the deposited energy of ionizing radiation in a cell result in the ejection of electrons from water $\left(\mathrm{H}_{2} \mathrm{O} \rightarrow \mathrm{H}_{2} \mathrm{O}^{+}+\mathrm{e}^{-}\right)$and that subsequent reactions lead to the formation of several reactive species like $\mathrm{e}_{\mathrm{aq}}^{-}, \mathrm{HO}^{\bullet}, \mathrm{H}^{\bullet}, \mathrm{H}_{2}$, or $\mathrm{H}_{2} \mathrm{O}_{2}$ [104. Most of the radicals will react well within $1 \mu$ s due to their instability, but superoxide $\left(\mathrm{O}_{2}^{-}\right)$and hydrogen peroxide $\left(\mathrm{H}_{2} \mathrm{O}_{2}\right)$ are comparatively stable and can diffuse within the cell leading to sample damage at sites different from the exposed area 104 .

In living cells, ionizing radiation induces mutation, cell transformation or causes cell death. In this context the radiation damage to the DNA in form of single and double strand breaks is particularly severe [107]. However, the radiation can also affect other biological molecules in the cell like for instance membrane lipids, which might lead to a disturbance of the membrane function and a loss of compartmentalization [104. The lethality of mammalian cells upon irradiation has been addressed in various studies 108 113. In most mammalian cell populations, an exposure to a dose of 10 Gy X-rays causes more than $90 \%$ cell killing [107]. However, by the addition of radical scavengers it is possible to increase the surviving fraction of cells [109, 110]. Furthermore, bystander cell killing has been observed in experiments, in which only one cell in a $5 \times 5 \mathrm{~mm}^{2}$-sized culture dish had been irradiated [113].

To relate the values for the radiation doses discussed above to doses encountered in medical applications, it should be noted that for instance the average effective dose applied for a radiography of the knee is $0.005 \mathrm{mSv}$ and for a computer tomography of the head is $2 \mathrm{mSv}$ with the unit $\mathrm{Sv}=\mathrm{J} / \mathrm{kg}$ [114. Note that a conversion factor of "one" between the absorbed dose in Gray and the effective dose in Sievert is employed for X-rays.

\subsection{Synchrotron Set-ups}

Synchrotron measurements were performed at three different beamlines at different synchrotron facilities: the cSAXS beamline at the Swiss Light Source (SLS) at the Paul Scherrer Institute (PSI, Villigen, Switzerland), the ID13 beamline at the European Synchrotron Radiation Facility (ESRF, Grenoble, France), and the P10 beamline at the storage ring PETRA III (HASYLAB, DESY, Hamburg, Germany). 
In this section, the different set-ups, from which data are presented in this thesis, are described. A summary of the experimental parameters of the set-ups during the different beamtimes is presented in Tab. 3.1.

\begin{tabular}{lcllll}
\hline set-up & $E[\mathrm{keV}]$ & $I_{0}[\mathrm{cps}]$ & $f_{h} \times f_{v}\left[\mathrm{~nm}^{2}\right]$ & $d[\mathrm{~m}]$ & $q_{\min }, q_{\max }\left[\mathrm{nm}^{-1}\right]$ \\
\hline cSAXS, May 2012 & 8.7 & $7.3 \times 10^{8 *}$ & $215 \times 165^{\dagger}$ & 7.543 & $0.041,1.204$ \\
cSAXS, Feb. 2013 & 8.7 & $2.8 \times 10^{8 *}$ & $145 \times 225^{\dagger}$ & 2.163 & $0.044,4.167$ \\
ID13, June 2011 & 15.25 & $3.0 \times 10^{9}$ & $140 \times 110$ & 0.8841 & $0.183,1.755$ \\
ID13, Nov. 2011 & 15.26 & $3.0 \times 10^{9}$ & $200 \times 125$ & 0.9303 & $0.142,1.673$ \\
ID13, Nov. 2012 & 14.92 & $8.7 \times 10^{9}$ & $160 \times 100$ & 0.9511 & $0.114,2.347$ \\
P10, Oct. 2012 & 7.9 & $1.5 \times 10^{11}$ & $390 \times 175$ & 5.22 & $0.081,0.521$ \\
P10, March 2013 & 7.9 & $1.1 \times 10^{11}$ & $700 \times 260$ & 5.22 & $0.090,0.532$ \\
\hline
\end{tabular}

Tab. 3.1.: List of set-up parameters during the different beamtimes. $E$ is the X-ray photon energy, $I_{0}$ the primary beam intensity, $f_{h}$ and $f_{v}$ are the focus sizes (FWHM) in horizontal and vertical direction, $d$ the sample-to-detector distance. Further, $q_{\min }$ is the minimum measurable $q$-value, which is limited by the extension of the primary beam or the beamstop, and $q_{\max }$ is the maximum detectable $q$-value, which is limited by the detector size and distance. ${ }^{*}$ Determined for a slit size sl0wh $=0.04$ and ${ }^{\dagger}$ for a slit size sl0wh $=0.02$, see description of the set-up at cSAXS beamline in section 3.2 .3 .

\subsubsection{ID13 Beamline, ESRF}

At the ID13 beamline at the ESRF, the measurements were performed in the experimental hutch III providing a nano-focused beam. The undulator beam was monochromatized by a channel-cut $\mathrm{Si}(111)$ monochromator to a photon energy of around $15 \mathrm{keV}$ (compare Tab. 3.1 for the exact parameters at each beamtime) and pre-focused by refractive beryllium lenses. Fig. 3.3 shows a sketch of the set-up in the experimental hutch III. The beam was focused on the sample by nanofocusing parabolic refractive X-ray lenses [22, 115- 117] and cleaned by an electron microscopy aperture with a diameter of $20 \mu \mathrm{m}$ and a pin hole, yielding a spot size of about $150 \times 150 \mathrm{~nm}^{2}$ (horizontal $\times$ vertical) and a primary beam intensity of about $3 \times 10^{9}$ cps (compare Tab. 3.1).

The samples were mounted on a scanning stage comprising a hexapod for coarse sample positioning and a piezoelectric stage for fine translations during scans. Behind the sample, the primary beam was blocked by a beamstop with a diameter of about $80 \mu \mathrm{m}$ and the scattered intensity was recorded using a Maxipix detector (ESRF, Grenoble, France) with a resolution of $516 \times 516$ pixels and a pixel size of 


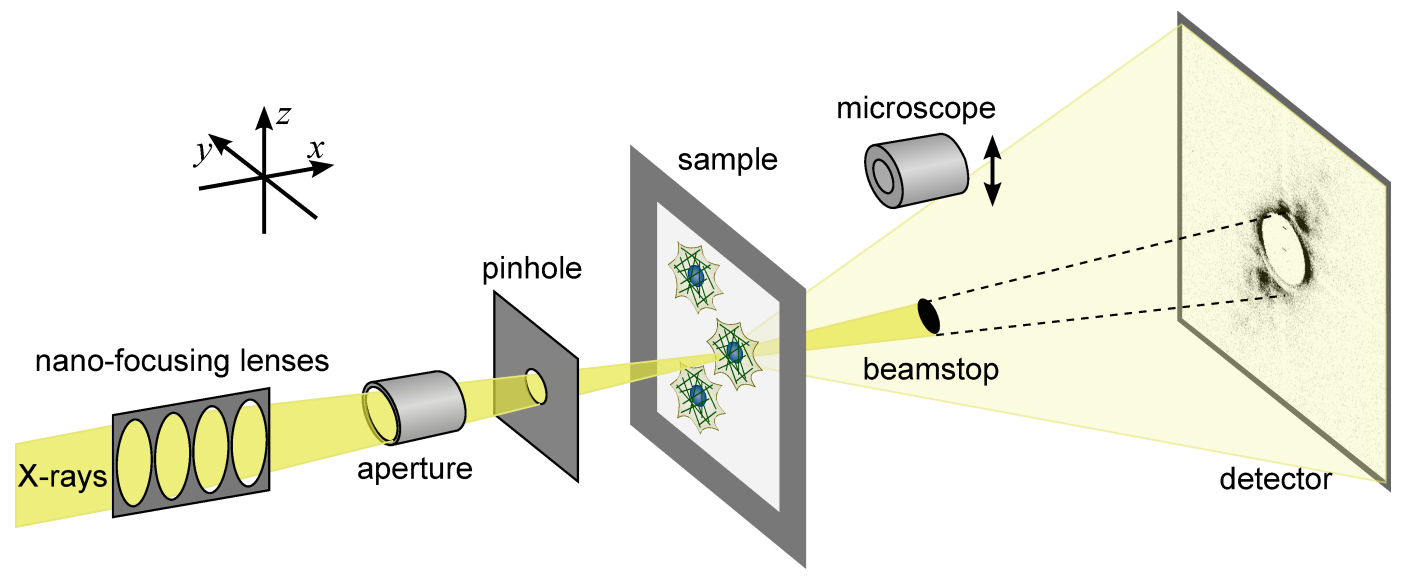

Fig. 3.3.: Sketch of the set-up at experimental hutch III at the ID13 beamline. The monochromatic beam was focused by nano-focusing parabolic refractive X-ray lenses on the sample. The beam was cleaned by an electron microscopy aperture and a pin hole. Behind the sample, the primary beam was blocked by a beamstop and the scattered intensity was recorded with a Maxipix detector. A visible light microscope could be moved into the beam path to align the sample prior to X-ray measurements. [Figure adapted from [39].]

$55 \times 55 \mu^{2}$ at a sample-to-detector distance of about $0.9 \mathrm{~m}$ (compare Tab. 3.1). For sample alignment prior to the X-ray measurements, a visible light microscope was moved into the beam path. The focus of the microscope was calibrated to coincide with the X-ray focus, which allowed for finding of the focal plane of the X-ray beam for all samples.

\subsubsection{P10 Beamline, PETRA III}

The Göttingen Instrument for Nano Imaging with X-rays (GINIX) [118 121] was employed for measurements at the coherence beamline P10 at the storage ring PETRA III. A detailed description of the set-up at the P10 beamline is given in the doctoral thesis by S. Kalbfleisch [121] and a simplified schematic of the set-up in the experimental hutch is presented in Fig. 3.4. The undulator beam was monochromatized to a photon energy of $7.9 \mathrm{keV}$ using a $\mathrm{Si}(111)$ double crystal. The beam was focused in horizontal and vertical directions by two Kirkpatrick-Baez (KB) mirrors to a size of about $500 \times 200 \mathrm{~nm}^{2}(\mathrm{FWHM})$ (compare Tab. 3.1 for the exact parameters at each beamtime). The beam sizes were measured by scanning a wave guide laterally through the beam. The parts of the incoming beam that were not reflected at both of the two mirrors were blocked by a pin hole. Further, the beam profile was cleaned by two apertures with inclined edges positioned upstream of the focus (first aperture: $20 \mu \mathrm{m}$ tantalum, size $70 \times 70 \mu \mathrm{m}^{2}$; second aperture: $10 \mu \mathrm{m}$ tantalum, size 


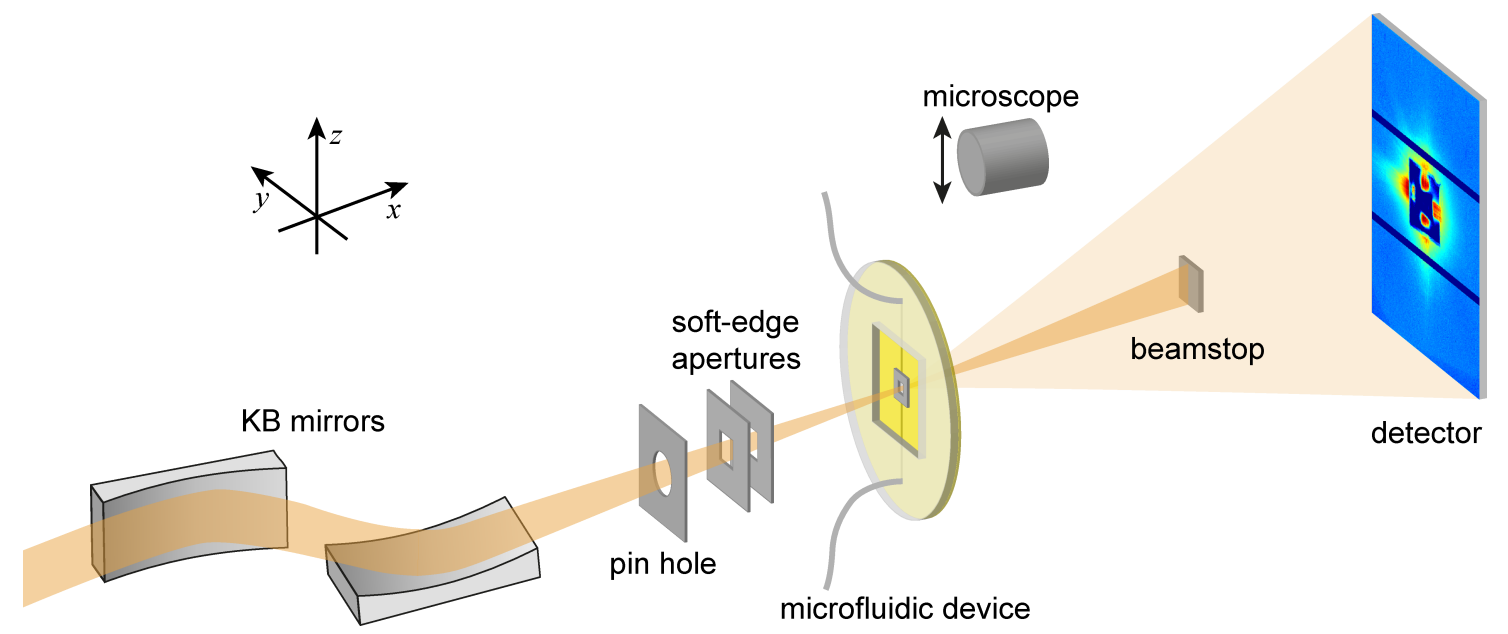

Fig. 3.4.: Schematic of the measurement set-up at the $\mathrm{P} 10$ beamline. The monochromatic X-ray beam was focused on the sample (here cells in a microfluidic device) using two Kirkpatrick-Baez (KB) mirrors. For blocking beams that were not reflected at both mirrors and cleaning of the beam profile, a pin hole and two soft-edge apertures were inserted into the beam path. Behind the sample, the primary beam was blocked by a beamstop and the scattered intensity was recorded using a Pilatus 300k detector. A visible light microscope could be moved into the beam path for sample alignment.

$\left.100 \times 100 \mu \mathrm{m}^{2}\right)$. This set-up yielded a primary beam intensity of about $1 \times 10^{11} \mathrm{cps}$ (compare Tab. 3.1).

The sample was mounted on a sample stage, including a piezoelectric stage allowing for precise translations, and aligned in the X-ray focus using a calibrated visible light microscope. Behind the sample, the primary beam was blocked by a beamstop $\left(100 \mu \mathrm{m}\right.$ tungsten, size $\left.800 \times 800 \mu \mathrm{m}^{2}\right)$ and the scattered radiation passed through an evacuated flight tube. The scattering signal was recorded on a Pilatus $300 \mathrm{~K}$ detector $\left(487 \times 619\right.$ pixels, pixel size: $172 \times 172 \mu \mathrm{m}^{2}$; Dectris Ltd., Baden, Switzerland $)$ at a sample-to-detector distance of $5.22 \mathrm{~m}$. Optionally, the primary beam intensity could be attenuated by the insertion of aluminum filters with a thickness increasing in steps of $100 \mu \mathrm{m}$.

\subsection{3. cSAXS Beamline, SLS}

The undulator beam was monochromatized by a $\mathrm{Si}(111)$ double crystal monochromator to a photon energy of $8.7 \mathrm{keV}$. A sketch of the set-up in the experimental hutch is depicted in Fig. 3.5. The beam was focused on the sample by a zone plate (diameter: $150 \mu \mathrm{m}$, outermost zone width: $100 \mathrm{~nm}$, thickness: $1 \mu \mathrm{m}$ gold). The first diffraction order was selected using a central stop (diameter: $40 \mu \mathrm{m}$, thickness: $15 \mu \mathrm{m}$ gold) and an order sorting aperture (OSA; diameter: $20 \mu \mathrm{m}$ ). At the 


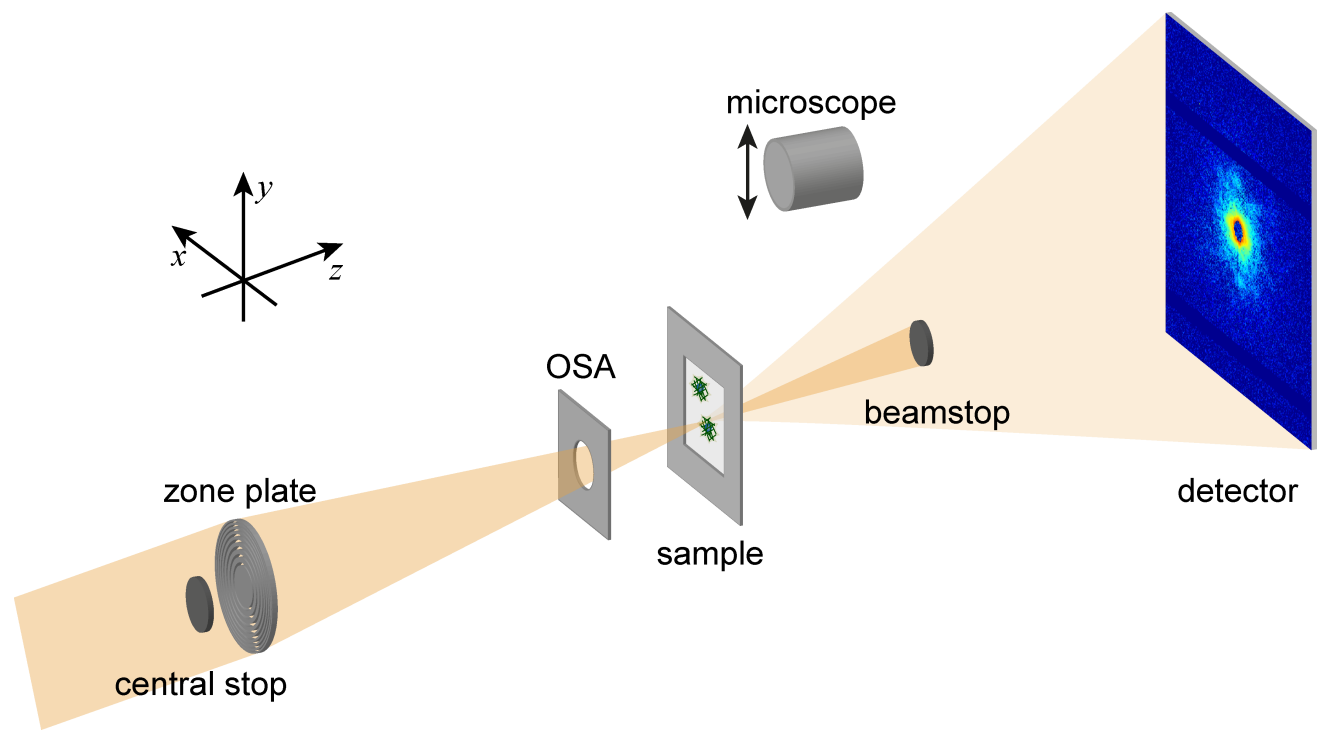

Fig. 3.5.: Schematic of the measurement set-up at the cSAXS beamline. The monochromatic X-ray beam was focused on the sample using a zone plate. The first diffraction order was selected using a central stop and an order sorting aperture (OSA). Behind the sample, the primary beam was blocked by a beamstop and the scattered intensity was recorded on a Pilatus $2 \mathrm{M}$ detector. A visible light microscope could be moved into the beam path for sample alignment.

beamtime in February 2013, a second central stop was inserted to assure complete absorption of the zeroth diffraction order. The opening of the horizontal front end slit (sl0wh) could be adjusted, which allowed for a regulation of the coherence of the beam and the primary beam intensity. For a slit opening of sl0wh $=0.02$, the focal spot size was determined using ptychography on a Siemens star test pattern yielding values of about $200 \times 200 \mathrm{~nm}^{2}$ (compare Tab. 3.1 for the exact parameters at each beamtime and appendix $\mathrm{E}$ for the results from ptychographic reconstructions). The slit opening was increased to sl0wh $=0.04$ for the diffraction measurements, which yielded a primary beam intensity of about $5 \times 10^{8} \mathrm{cps}$ in a slightly larger focal spot size (not measured), which was still small enough to assure probing the local sample structure.

The sample was mounted on a piezoelectric stage, which allowed for precise translations during the scans, and aligned in the X-ray focus using a calibrated visible light microscope. Behind the sample, the radiation passed through a flight tube (length of about $7 \mathrm{~m}$ and filled with helium in May 2012; length of about $2 \mathrm{~m}$ and evacuated in February 2013) and the primary beam was optionally blocked by a beamstop in the flight tube. The scattered intensity was recorded on a Pilatus $2 \mathrm{M}$ detector $\left(1475 \times 1679\right.$ pixels, pixel size: $172 \times 172 \mu \mathrm{m}^{2}$; Dectris Ltd., Baden, Switzerland $)$. Optionally, the primary beam intensity could be attenuated by the insertion of sili- 
con and germanium filters with different thicknesses.

Note that the coordinate systems for the measurements at the cSAXS beamline was defined differently to the one at the ID13 beamline and the P10 beamline, i.e. the beam direction defines the $z$-coordinate and the sample is scanned in the $x$-y-plane. This convention is also used for the displayed scattering patterns and real space images in the following chapters.

\subsubsection{Comparison of the Different Set-Ups}

Several specific aspects of the different set-ups affected the measurement strategies as well as the data quality. The result from the measurements at the different setups are presented in the chapters 577. In this section, a short comparison of the set-ups with regard to the effects on the data recording and analysis will be given. An important difference between the set-ups was the focusing optic. At the cSAXS beamline, the beam was focused using a zone plate. At the P10 beamline a pair of KB mirrors was employed and at the ID13 beamline nano-focusing lenses were used for focusing. A general description and discussion of different focusing optics for hard X-rays can be found in [122,123]. The focusing optics (in combination with other influencing factors) affect the photon flux, the focus size and the cleanliness of the beam profile. The difference in the photon flux on the sample is one of the most important aspects. However, the photon flux is not only related to the properties of the focusing optics, but also to the general properties of the facility. A higher photon flux allows for a reduction of the exposure time and yields a better signalto-noise ratio in the data. In particular for the measurements on hydrated samples, a high photon flux is required to obtain a high signal-to-noise level in the scattering patterns due to the low electron density contrast in the sample and the absorption of the water layer surrounding the sample. The highest photon flux was available at the P10 beamline (compare Tab. 3.1). Depending on the specific experiment, a small focus size might be required to assure a small probe volume. Here, the beam size available at the P10 beamline might be too large for specific applications aiming at a probe area below $200 \times 200 \mathrm{~nm}^{2}$. For the analysis of the scattering patterns, the cleanliness of the beam profile is an important issue. The cleanest beam profile was available at the ID13 beamline. Here, comparatively weak scattering streaks due to the aperture downstream of the nano-focusing lenses were observed. At the cSAXS beamline, strong scattering from the OSA was observed and could not be reduced by choosing a different OSA or insertion of a second central stop. At the P10 beamline, strong streaks from the edges of the KB mirrors were present, which could be reduced by the insertion of two soft-edge apertures in a similar fashion as presented by Takahashi et al. [124], but not completely eliminated. 
The overhead scan time due to the motor movements during the scan and the total scan time are as well important for the measurements (compare chapters 577). At the cSAXS beamline and at the P10 beamline, a continuous scanning mode was available, in which the motor of the fast scanning axis moved continuously in one direction. By employing this scanning mode, a reduction of the total scan time by a factor of eight could be achieved.

Concluding from this comparison, all of the three set-ups have different strengths and weaknesses. Therefore, the most import demands of an experiment on a set-up must be defined and the set-up must be chosen appropriately. 



\section{Biological System and Sample Preparation}

In this chapter the preparation of cellular samples for synchrotron experiments is described. First, experiments were performed on samples of plunge-frozen and freezedried cells on silicon nitride membrane windows. In a second step, experiments were performed on fixed-hydrated as well as living cells. For these experiments sample environments based on microfluidic devices that are both compatible with X-ray scattering experiments as well as cell culture were developed and the fabrication pathway of these devices is shown. Parts of this chapter have been published in Lab on a Chip [40].

\subsection{Cell Line and Culture}

As a model system the cell line SK8/18-2, generously provided by Rudolf Leube (RWTH Aachen, Germany), was used. These cells were derived from human adrenal cortex carcinoma SW-13 cells (ATCC CCL-105) [125], which were stably transfected with DNA encoding for fluorescent human keratin hybrids (HK8-CFP, HK18YPF) 62, 63, 68 allowing for imaging of the keratin network using fluorescence microscopy.

The cells were cultured in high glucose (4.5 g/l) Dulbecco's Modified Eagle Medium (DMEM; PAA Laboratories GmbH, Pasching, Austria) with 10\% fetal calf serum (FCS, Invitrogen, Darmstadt, Germany), $100 \mathrm{U} / \mathrm{ml}$ penicillin and $0.1 \mathrm{mg} / \mathrm{ml}$ streptomycin (Pen-Strep; Sigma-Aldrich, Taufkirchen, Germany) at $37^{\circ} \mathrm{C}$ in a water saturated atmosphere with $5 \% \mathrm{CO}_{2}$. The cells were passaged every $3-4$ days. For passaging, the cells were first washed with phosphate buffered salin $\mathrm{I}^{1}$ (PBS) supplemented with $0.02 \%$ (w/v) ethylenediaminetetraacetic acid (EDTA; Roth, Karlsruhe, Germany) and then incubated with trypsin/EDTA solution $(0.25 \%(\mathrm{v} / \mathrm{v})$ trypsin (Sigma-Aldrich), $0.02 \%$ (w/v) EDTA in PBS) for about $2 \mathrm{~min}$. The trypsin/EDTA

${ }^{1} \mathrm{PBS}$ was prepared by dissolving $0.137 \mathrm{M} \mathrm{NaCl}, 2.7 \mathrm{mM} \mathrm{KCl}, 4.3 \mathrm{mM} \mathrm{Na}_{2} \mathrm{HPO}_{4}, 1.4 \mathrm{mM} \mathrm{KH}_{2} \mathrm{PO}_{4}$ in ultra-pure water yielding a $\mathrm{pH}$ of 7.2 
solution was removed and the cells were suspended in culture medium and distributed on petri dishes in the desired dilution.

\subsection{Preparation of Cellular Samples for Synchrotron Experiments}

The first experiments were performed on samples of plunge-frozen and freeze-dried cells on silicon nitride membrane windows. This sample type had the advantage that they are easy to transport and to handle at a beamtime, meaning that they did not need to be kept under cryo-conditions or at physiological conditions. Since the samples were dry, the electron density contrast between cellular material (mainly proteins, lipids, DNA) and air as a surrounding medium was about $\Delta \rho_{\text {dry }}=0.4 \mathrm{e}^{-} / \AA^{3} \quad 126$, which led to a comparatively strong scattering signal. However, we observed structural changes like cracks on the scale of micrometers in some cells after plunge-freezing and freeze-drying, and additional alterations on small length scales, which were unobservable with visible light microscopy, could not be excluded.

To study the native cellular structure in hydrated cells, in a second step, experiments were performed on fixed-hydrated as well as living cells. For these samples the preparation included fewer different steps, but the choice of an appropriate sample environment was a challenging task (see section 4.3) and the use of microfluidic devices imposed further restrictions on the set-up and the measurements. The electron density contrast between cellular material and water or buffer as a surrounding medium was about $\Delta \rho_{\text {hyd }}=0.1 \mathrm{e}^{-} / \AA^{3}[126$, which is a factor of four lower than the electron density contrast for dry samples and therefore leading to a weaker scattering signal. Furthermore, for living samples of the genetically modified SK8/18-2 cells, biosafety issues needed to be considered and therefore the samples could not be measured during some of the beamtimes.

In the following section, the different steps during the preparation of different sample types are described and a schematic is presented in Fig. 4.1.

\subsubsection{Growing Cells on Silicon Nitride Membrane Windows}

For all experiments silicon nitride $\left(\mathrm{Si}_{3} \mathrm{~N}_{4}\right)$ membrane windows (Silson Ltd, Blisworth, England) with a frame size of $5 \times 5 \mathrm{~mm}^{2}$, frame thickness of $200 \mu \mathrm{m}$, membrane size of $1.5 \times 1.5 \mathrm{~mm}^{2}$ and membrane thickness of $200-1000 \mathrm{~nm}$ were used as growth substrate for the cells. The $\mathrm{Si}_{3} \mathrm{~N}_{4}$ membrane windows were taken out of the transport tube with fine tweezers and one corner on the non-flat side of the window was marked 


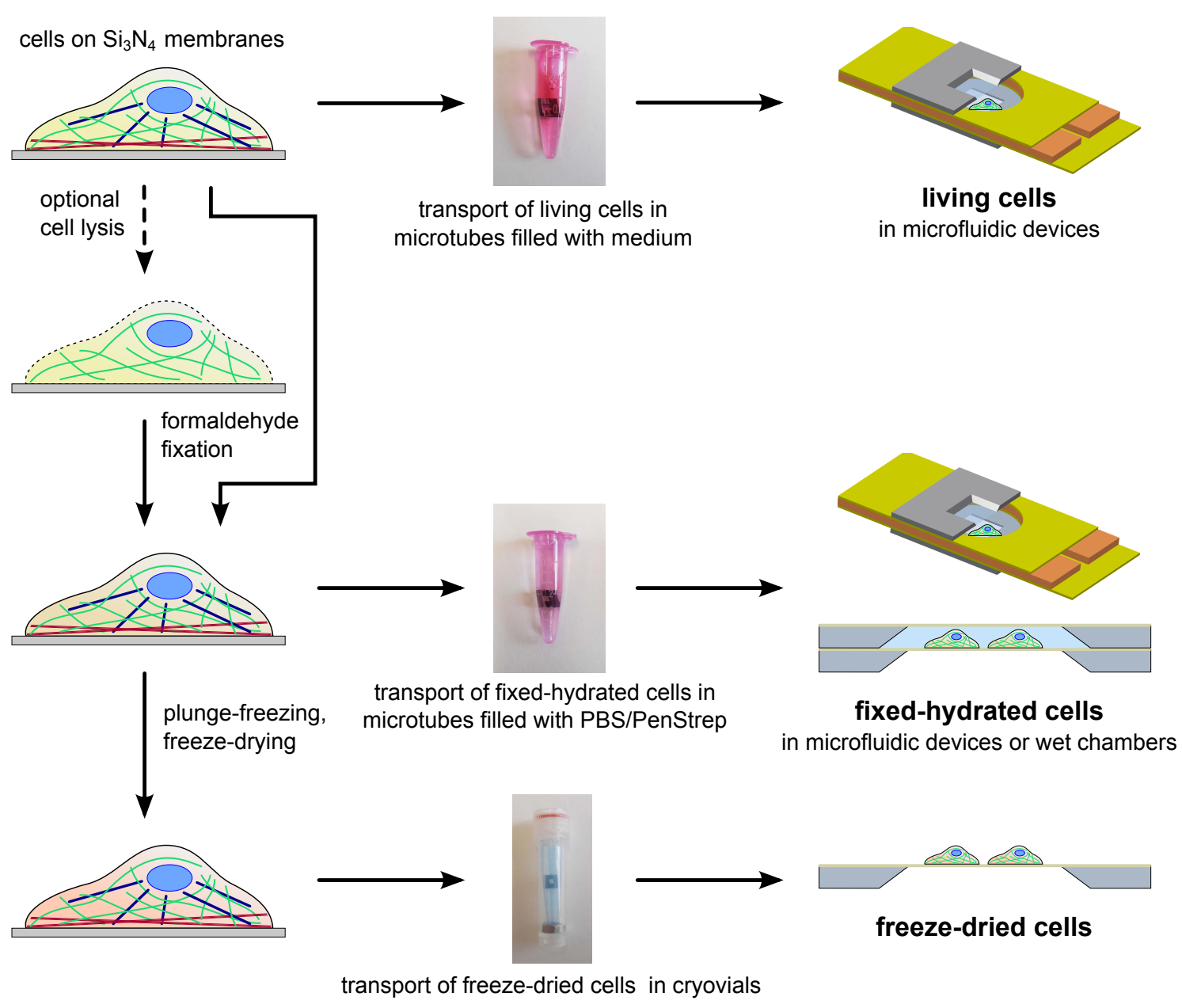

Fig. 4.1.: Schematic of the preparation pathway of different sample types for synchrotron experiments. For all samples the cells were grown on silicon nitride membrane windows. Living cells were kept in medium during transport to the synchrotron. For fixed samples the cells were fixed with formaldehyde solution and stored in buffer (fixed-hydrated samples) or plunge-frozen and freeze-dried.

with a pen. The windows were placed with the flat side pointing upwards in a petri dish and they were treated with oxygen plasma (PDC-32G-2, Harrick Plasma, Ithaca, US) for $30 \mathrm{~s}$ at the level "high" to render the surface hydrophilic. At the clean bench in the cell culture laboratory, PBS was added to the dish with the window, which allows for an easier removal of the windows from the dish. The windows were transferred to petri dishes $(5 \mathrm{~cm}$ diameter) with cell culture medium with the flat side facing upwards and incubated for 5 min to allow for the adsorption of FCS proteins to the surface, thereby providing anchoring points for the cells. Air bubbles below the window should be avoided and, if possible, removed.

The SK8/18-2 cell were suspended in medium at a concentration of about $10^{6}$ cells $/ \mathrm{ml}$ and $0.5 \mathrm{ml}$ cell suspension was added to the dish with the windows. The cells were 
incubated for $1-2$ days at $37^{\circ} \mathrm{C}$ in a water saturated atmosphere with $5 \% \mathrm{CO}_{2}$.

\subsubsection{Living Cells}

For the preparation of living cellular samples, the cells were grown on $\mathrm{Si}_{3} \mathrm{~N}_{4}$ membrane windows as described in section 4.2.1. The cells were packaged in microtubes (total volume $650 \mu \mathrm{l}$; VWR, Darmstadt, Germany) with $600 \mu \mathrm{l}$ warm and freshly opened culture medium and the tubes were sealed with parafilm to maintain the physiological pH. The cells were kept in these tubes until the measurement (ID13, November 2012) or taken out of the tubes and kept in dishes with culture medium (DMEM with $10 \%$ FCS and Pen-Strep) in a cell incubator at $37^{\circ} \mathrm{C}$ in a water saturated atmosphere with $5 \% \mathrm{CO}_{2}$ (P10, March 2013). The cells could be kept in the sealed tube at room temperature for about two days without major morphological changes and even after four days cells with a normal morphology could be identified. For the measurements, the $\mathrm{Si}_{3} \mathrm{~N}_{4}$ membrane windows with the cells were incorporated in microfluidic devices as described in section 4.3.2.3.

\subsubsection{Fixed-Hydrated Cells}

The cells were grown on $\mathrm{Si}_{3} \mathrm{~N}_{4}$ membrane windows as described in section 4.2.1 for 1-2 days. Afterwards the cells were briefly washed with PBS and fixed by adding $3.7 \%$ formaldehyde solution (diluted from $37 \%$ formaldehyde solution supplemented with 10\% methanol as stabilizer; Sigma-Aldrich) for 15-20 min at room temperature. The samples were washed three times with PBS and stored in PBS supplemented with $100 \mathrm{U} / \mathrm{ml}$ penicillin and $0.1 \mathrm{mg} / \mathrm{ml}$ streptomycin (PBS/Pen-Strep). Phase contrast and epifluorescence microscopy images of the whole window were taken at an inverted microscope (IX71, Olympus, Hamburg, Germany), which was equipped with $10 \times$ and $20 \times$ objectives. The sample windows were packaged in microtubes filled with PBS/Pen-Strep and the tubes were sealed with parafilm.

\subsubsection{Freeze-Dried Cells}

The cells were grown on $\mathrm{Si}_{3} \mathrm{~N}_{4}$ membrane windows and fixed with formaldehyde solution as described in section 4.2.1 and 4.2.3. Phase contrast and epifluorescence microscopy images of the whole window were taken at an inverted microscope (IX71; Olympus, Hamburg, Germany), which was equipped with $10 \times$ and $20 \times$ objectives. If necessary the samples could be stored in PBS/Pen-Strep for several weeks before further preparation.

For plunge-freezing of the samples, a commercial set-up (Leica EM GP grid plunger, 


\begin{tabular}{lll}
\hline & melting point & boiling point \\
\hline ethane & $-183.27^{\circ} \mathrm{C}$ & $-88.62^{\circ} \mathrm{C}$ \\
propane & $-187.69^{\circ} \mathrm{C}$ & $-42.07^{\circ} \mathrm{C}$ \\
nitrogen & $-209.86^{\circ} \mathrm{C}$ & $-195.8^{\circ} \mathrm{C}$ \\
ethane/propane & $-196^{\circ} \mathrm{C}$ & \\
\hline
\end{tabular}

Tab. 4.1.: Boiling and melting points of ethane, propane, nitrogen and a mixture of $37 \%$ ethane and $63 \%$ propane. The data were taken from [127, 128].

Leica, Vienna, Austria) was used (see Fig. 4.2). As cryogen either liquid ethane or a liquid ethane/propane mixture (37.5\% ethane) cooled by liquid nitrogen was employed. The cryogen needs to facilitate high cooling rates to maintain the native sample structure by immobilization of water molecules in the sample as amorphous and non-crystalline structures (vitrification of the sample) [129]. These cooling rates are provided by liquid ethane or a liquid ethane/propane mixture at temperatures of $-186^{\circ} \mathrm{C}$ or $-194^{\circ} \mathrm{C}$, respectively 127,129 . Using the ethane/propane mixture has the advantage that it stays liquid even at the temperature of liquid nitrogen, whereas pure ethane freezes and needs to be melted before plunging (compare Tab. 4.1). Liquid nitrogen itself is not an appropriate cryogen, since upon contact with a sample having an ambient temperature a thin vapor layer forms between the sample and the nitrogen, which insulates and therefore prevents further rapid cooling of the sample [129.

The grid plunger was prepared for plunge-freezing by filling the ultrasonic vaporizer for regulation of the relative humidity in the sample chamber with ultra-pure water, filling liquid nitrogen in the nitrogen reservoir and filling the cryogen in the cryogen container. The set-points as shown in table 4.2 were selected in the controlling software. Cryovials (see Fig. 4.3 ) and storage revolvers were cooled in liquid nitrogen (see Fig. 4.3d).

Before freezing, the fixed samples were washed three times with ultra-pure water to avoid salt precipitation on the membranes. The $\mathrm{Si}_{3} \mathrm{~N}_{4}$ membrane window was

\begin{tabular}{ll}
\hline$T_{\text {chamber }}$ & $20^{\circ} \mathrm{C}$ \\
$R H_{\text {chamber }}$ & $99 \%$ \\
$T_{\text {cryogen }}$ & $-186^{\circ} \mathrm{C}$ or $-194^{\circ} \mathrm{C}$ \\
\hline
\end{tabular}

Tab. 4.2.: Set-points for the temperature $T_{\text {chamber }}$ and the relative humidity $R H_{\text {chamber }}$ in the sample chamber and for the temperature $T_{\text {cryogen }}$ of liquid ethane or ethane/propane, respectively, during plunge-freezing with the Leica grid plunger. 


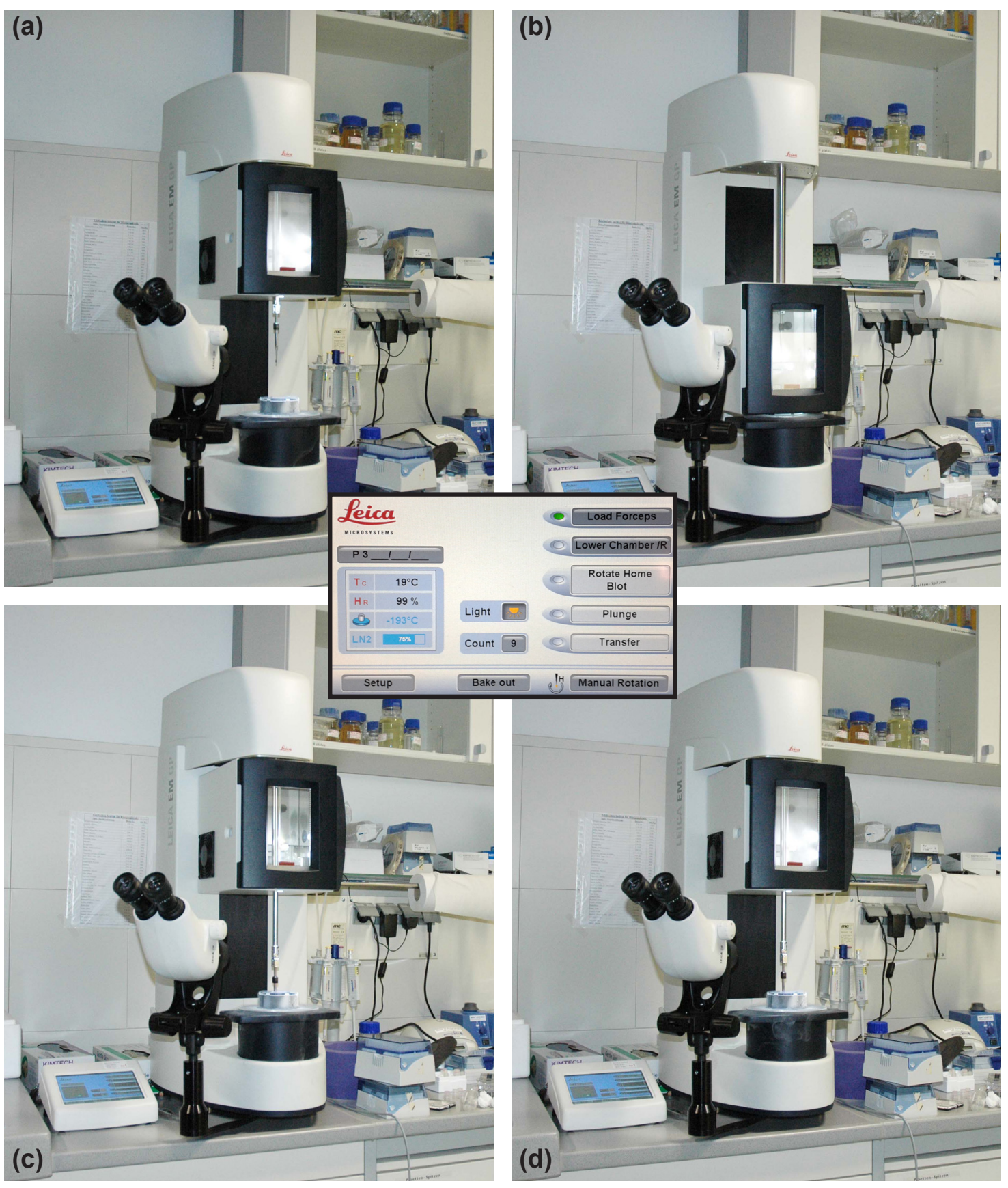

Fig. 4.2.: Photographs of the grid plunger in the different positions during plunge-freezing. (a) Load Forceps position, in which the sample can be attached to the plunging rod and the chamber is moved upwards. The inset shows the display of the controlling software. (b) Lower Chamber position, in which chamber is moved downwards and the sample is blotted. (c) Plunge position, in which the sample is plunged into the ethane bath and afterwards the chamber is mover upwards. (d) Transfer position, in which the sample is slightly lifted in the ethane bath allowing for the transfer to a cryovial. 
taken out of the water, grabbed with a pair of tweezers and mounted on the plunging rod of the grid plunger in the position Load Forceps. The different positions of the sample chamber and the plunging rod could be either selected manually in the controlling software or it could be switched to the next position by tripping the foot pedal. The sample chamber was lowered (position Lower Chamber) and the excess liquid on the sample was blotted manually with a paper wick (Mitegen, USA). Immediately after blotting, the sample was injected in the cryogen using the foot pedal (position Plunge). The sample was moved to the Transfer position, in which the window was located slightly above the cryogen surface, and the window was transferred to a cryovial filled with liquid nitrogen. The cryovial was transferred to

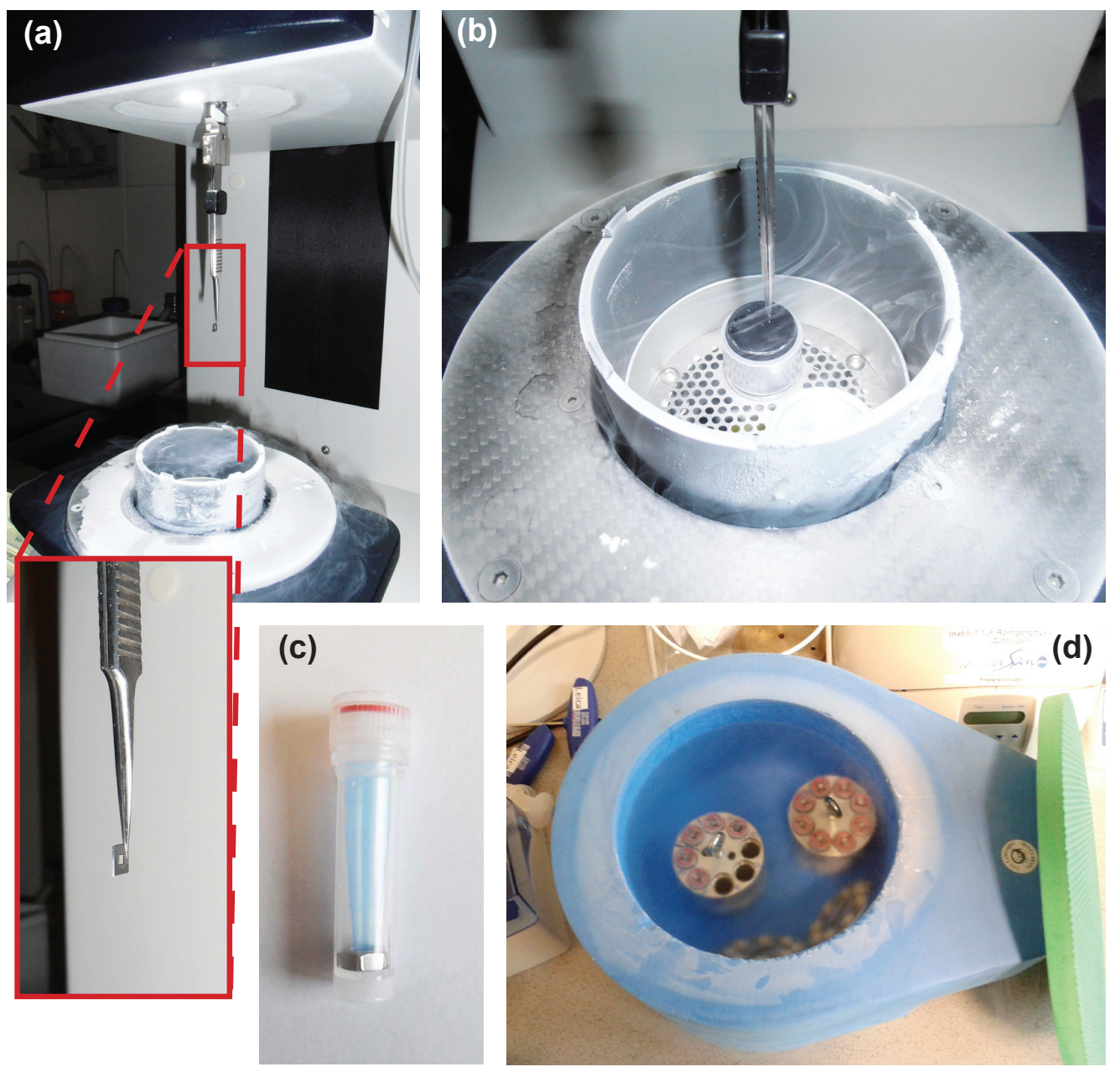

Fig. 4.3.: Photographs of the sample at different steps during plunge-freezing. (a) The sample was attached to the plunging rod in Load Forceps position. (b) After blotting the sample was plunged into liquid ethane (Plunge position) and transferred into a cryovial (c) in the Transfer position. The cryovial was kept in a storage revolver placed in liquid nitrogen $(d)$. 

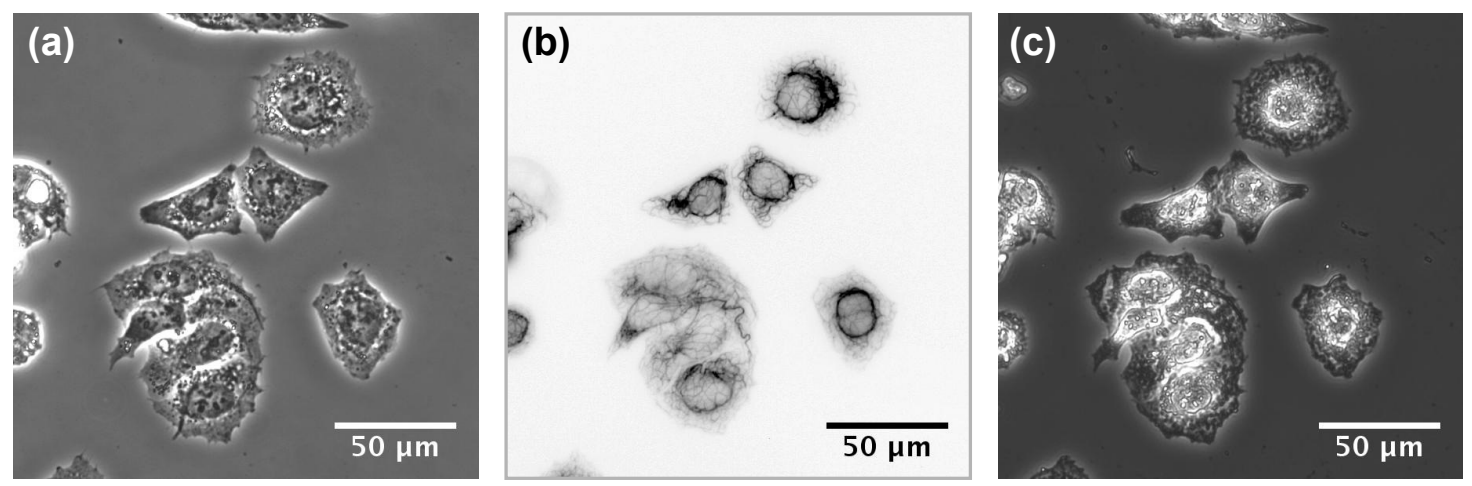

Fig. 4.4.: Microscopy images of a hydrated sample and the same sample after freezedrying. (a) Phase contrast image of a group of cells on a $\mathrm{Si}_{3} \mathrm{~N}_{4}$ membrane window and (b) fluorescence microscopy image of the keratin network. (c) Phase contrast image of the same group of cells after plunge-freezing and freeze-drying.

a storage revolver cooled in liquid nitrogen. All samples were stored for at least $24 \mathrm{~h}$ in liquid nitrogen to allow for ethane ice to evaporate and subsequently lyophilized in a home-built freeze-drier. During this step the frozen water in the sample was sublimated at pressures/temperatures below the triple point of water.

Afterwards, the samples were warmed up to room temperature and imaged again by phase contrast microscopy as shown in Fig. 4.4. The cell shape was very well conserved during the plunge-freezing and freeze-drying, and the $\mathrm{Si}_{3} \mathrm{~N}_{4}$ membrane was free from salt precipitates for all samples that were washed in ultra-pure water prior to plunge-freezing. The keratin network could not be imaged with fluorescence microscopy in the dry state, because the fluorophores were destroyed during sample preparation. Samples were stored over silica gel in a desiccator.

\subsubsection{Cellular IF Preparation}

The cytoskeleton of the SK8/18-2 cells consists not only of keratin, but also of microtubules and actin filaments, which might form bundles and thereby lead to similar scattering signals as keratin bundles. To simplify the data interpretation we exploited the fact that IFs are very stable and therefore it is possible remove all soluble proteins from the cytoplasm by detergent treatment of the cells without destroying the IF network. The detergent treatment also leads to a disassembly of filamentous actin (F-actin) and microtubules, which allows for washing out the monomeric subunits. However, during detergent treatment the IF network needs to be protected against protease activity by different kinds of protease inhibitor. The used protocol is a modification of the protocol described by Prahlad et al. 130] and was kindly provided by Melissa Mendez (Northwestern University, IL, USA). 


\begin{tabular}{ll}
\hline chemical & concentration \\
\hline EDTA & $5 \mathrm{mM}$ \\
EGTA & $5 \mathrm{mM}$ \\
$\mathrm{KCl}$ & $0.6 \mathrm{M}$ \\
Triton X-100 & $0.1 \%$ \\
Protease Inhibitor Cocktail & 1 tablet per $10 \mathrm{ml}$ \\
pepstatin & $10 \mu \mathrm{g} / \mathrm{ml}$ \\
PMSF & $1 \mathrm{mM}$ \\
TAME & $1 \mathrm{mM}$ \\
PBS $(10 \times)$ & $1 \mathrm{ml}$ per $10 \mathrm{ml}$ \\
\hline
\end{tabular}

Tab. 4.3.: Recipe of IF lysis buffer that was used for IF preparation. Due to instability in water the Protease Inhibitor Cocktail, pepstatin and PMSF were added immediately before use.

The lysis buffer was prepared according to the protocol presented in Tab. 4.3 and the components that are unstable in water (Protease Inhibitor Cocktail, pepstatin and PMSF) were added immediately before use. The cells were grown on $\mathrm{Si}_{3} \mathrm{~N}_{4}$ membrane window (or on cover slides for microscopy experiments) and incubated with IF lysis buffer for $15 \mathrm{~s}$. Subsequently the cells were washed three times with PBS and fixed for about $20 \mathrm{~min}$ with formaldehyde solution at room temperature or with cold methanol on ice. To test whether this protocol really leads to a disruption the actin filaments and microtubules in SK8/18-2 cells, fluorescence staining experiments for F-actin or microtubules in combination with staining of the DNA were performed on the cells that were treated with IF lysis buffer as well as on the untreated cells as control measurements. The employed fluorescence staining protocols are listed in appendix A. Fig. 4.5 shows examples for the fluorescence staining of F-actin in the native SK8/18-2 cells and in the SK8/18-2 cells treated with IF lysis buffer. Whereas the actin cortex and stress fibers are visible in the native cells, there are no distinct structures visible in the cells that were treated with IF lysis buffer.

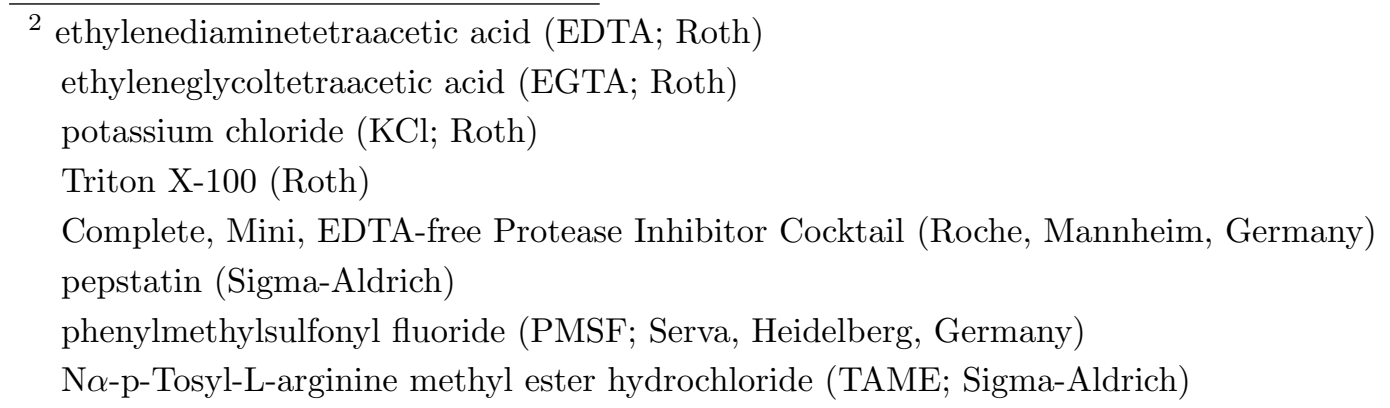


native cells

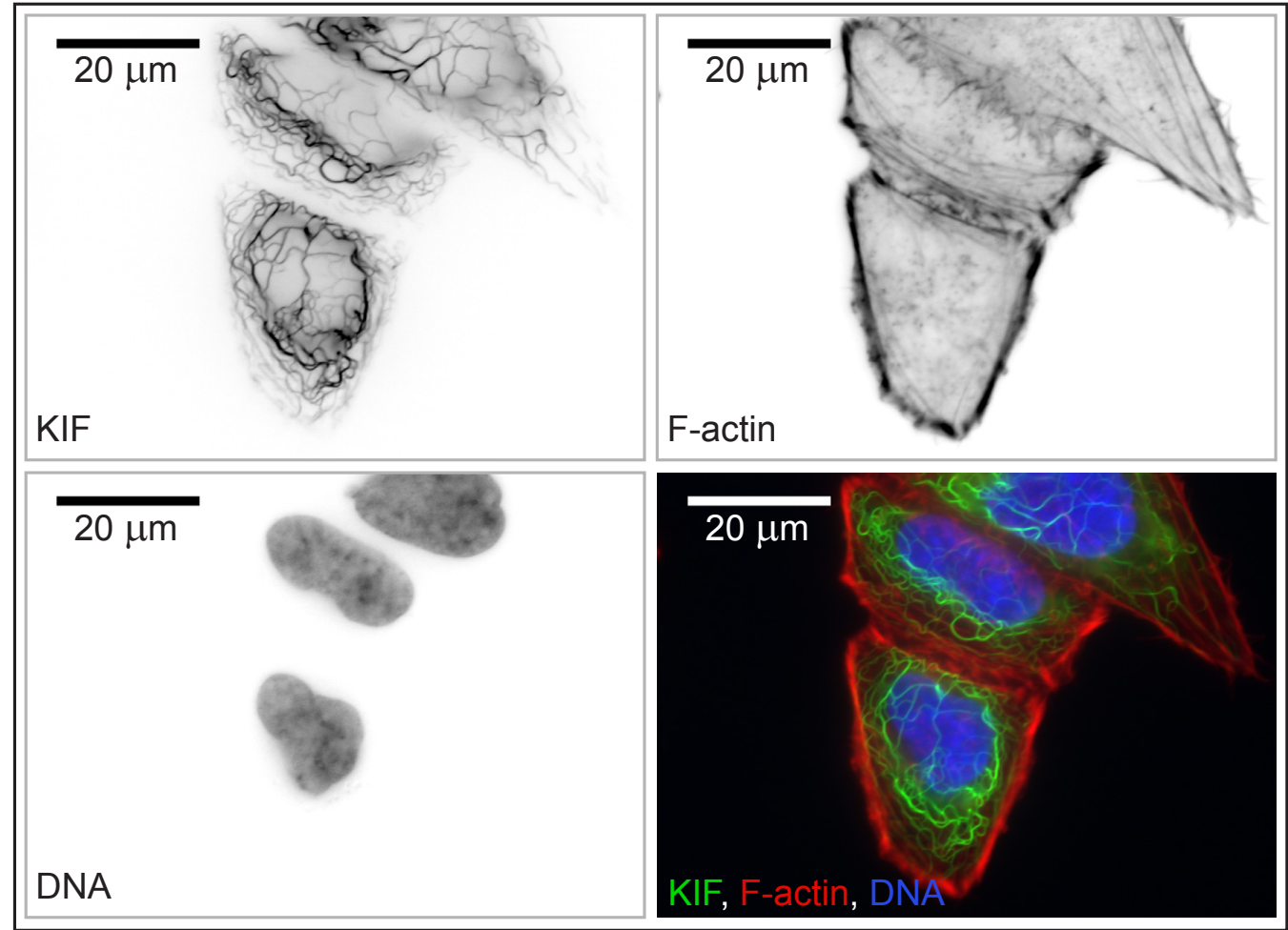

cells treated with IF lysis buffer

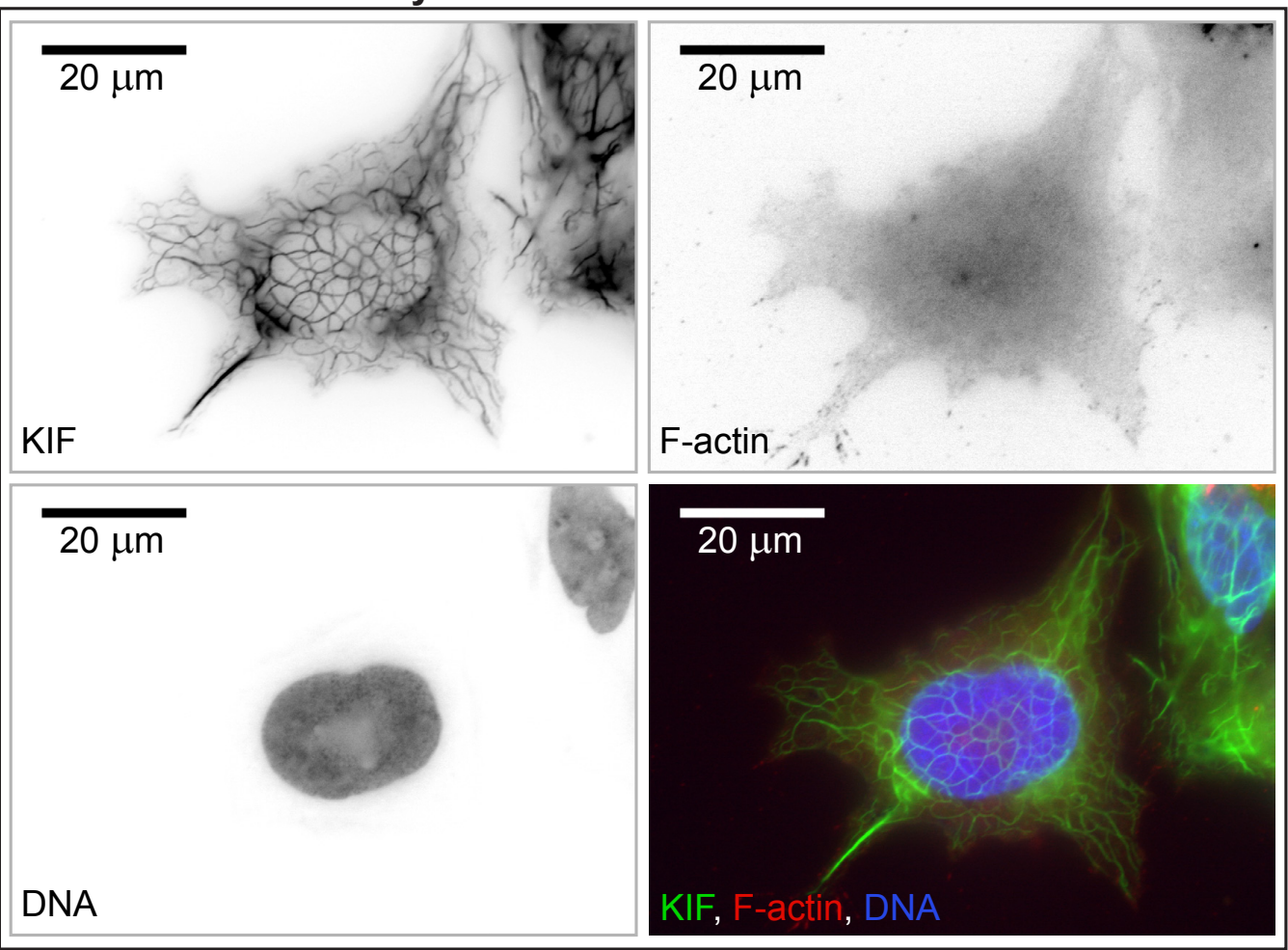

Fig. 4.5.: Fluorescence staining of F-actin and DNA in native SK8/18-2 cells (top) and in SK8/18-2 cells treated with IF lysis buffer (bottom). 
native cells

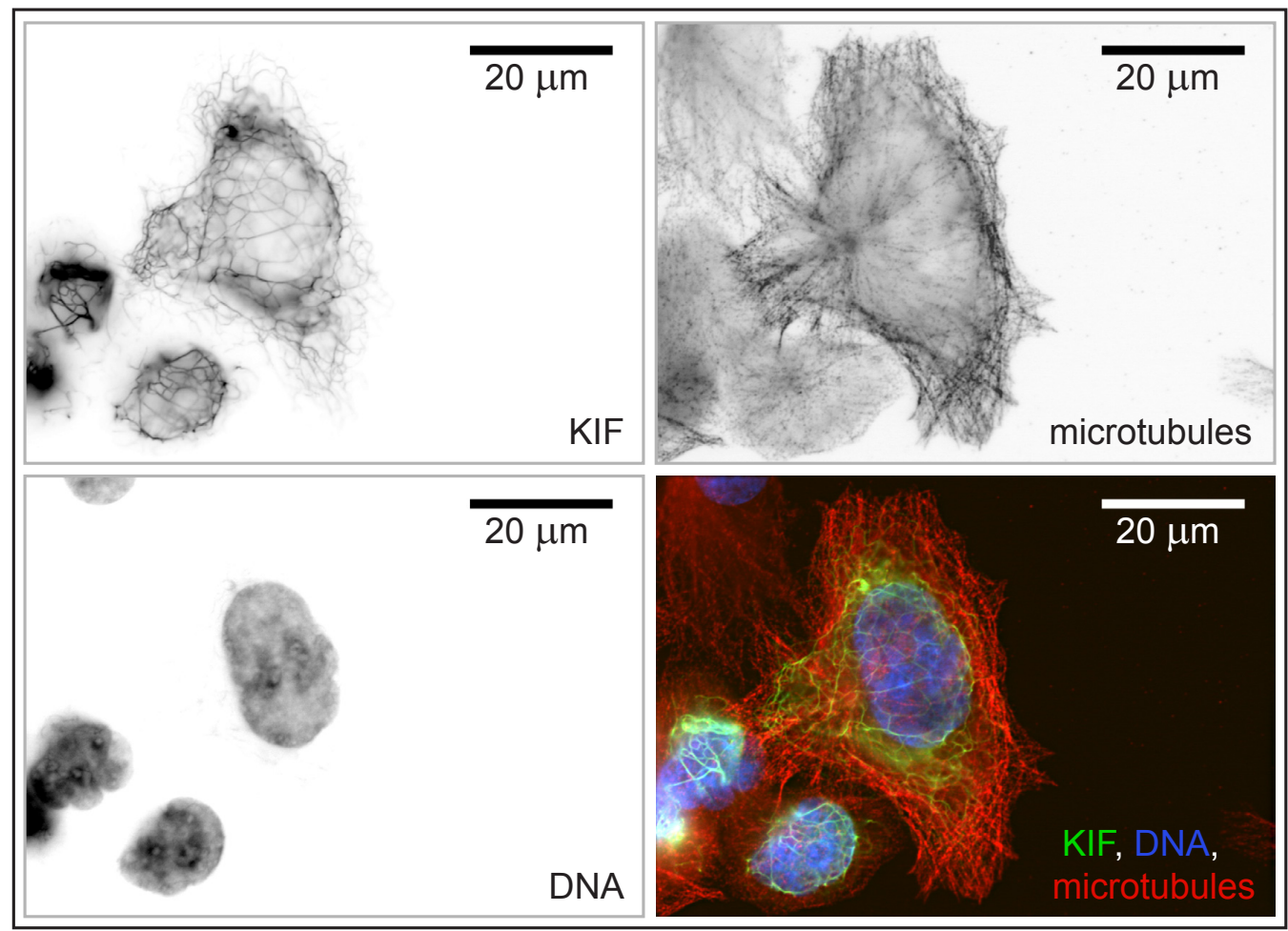

cells treated with IF lysis buffer

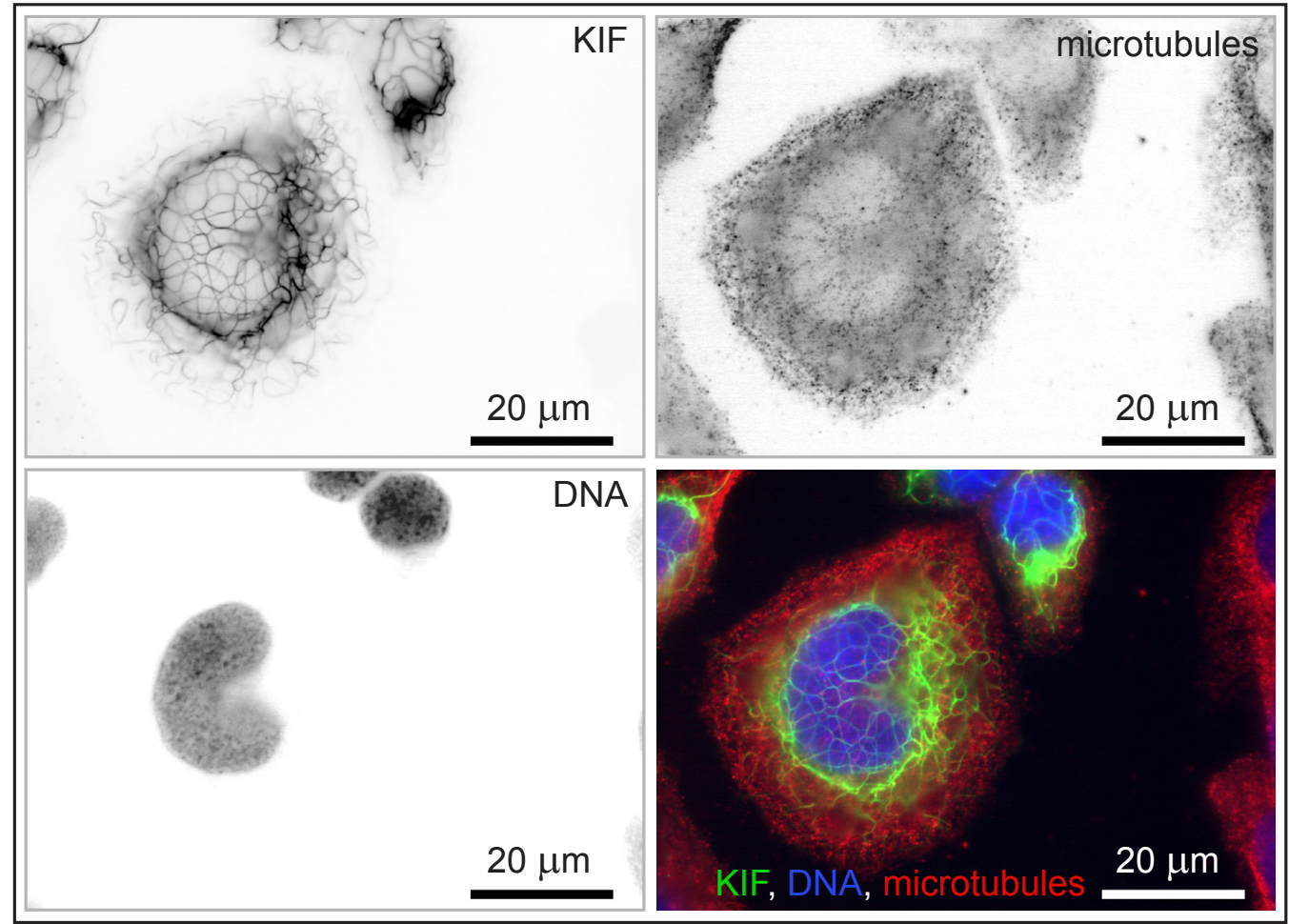

Fig. 4.6.: Fluorescence staining of microtubules and DNA in native SK8/18-2 cells (top) and in SK8/18-2 cells treated with IF lysis buffer (bottom). 
Examples for immunofluorescence staining of microtubules in the native SK8/18-2 cells and in the SK8/18-2 cells treated with IF lysis buffer are shown in Fig. 4.6. In the native cells microtubules are visible as filamentous structures, whereas only a diffuse or point-like fluorescence signal is visible in the cells that were treated with IF lysis buffer.

Based on the presented results, treatment of cells with IF lysis buffer leads to a disruption of F-actin and microtubules while the KIF network is preserved. However, the disruption and removal of $\mathrm{F}$-actin and microtubules was not always as perfect as shown in Fig. 4.5 and 4.6 . For some cells there were remaining actin filaments and microtubules visible after treatment with IF lysis buffer (not shown) and therefore it could not be completely guaranteed that in all cells only the KIF network was remaining. Furthermore, after treatment with IF lysis buffer, substructures could be identified in the cytoplasm using bright-field microscopy, as shown in Fig. 4.7 for a SK8/18-2 cell, which was treated with IF lysis buffer and was also imaged using fluorescence microscopy (see Fig. 4.5. bottom). Therefore it is not clear, to which extent cellular organelles are destroyed or removed from the cell. Another drawback from treatment with the lysis buffer was that the fluorescence signal from the keratin network was weaker after incubation with IF lysis buffer, which might be attributed to a cleavage of the fluorescent proteins (CFP, YFP) off from the KIFs by proteases or to a denaturation of the fluorescent proteins in the lysis buffer.

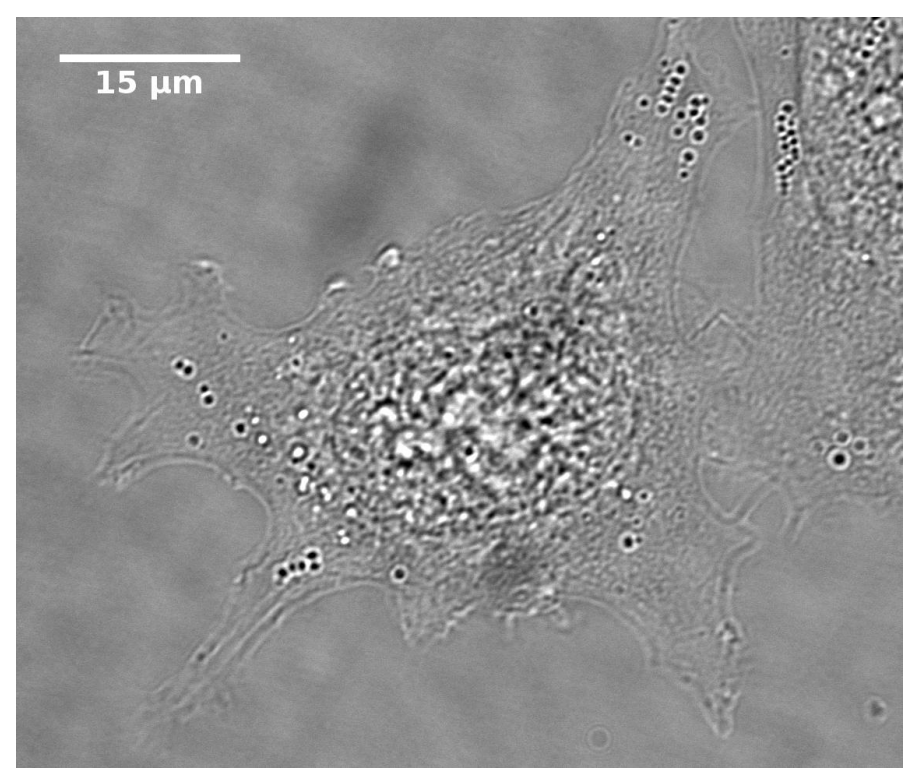

Fig. 4.7.: Bright-field microscopy image of SK8/18-2 cell treated with IF lysis buffer. The fluorescence microscopy images of this cell are presented in Fig. 4.5 (bottom). 


\subsection{Sample Environments for Hydrated Cells}

The design and the fabrication of a sample environment that is both compatible with culturing of living adherent cells as well as performing X-ray scattering experiments on these cells, is a challenging step. As a first approach we used simple wet chambers built of two $\mathrm{Si}_{3} \mathrm{~N}_{4}$ membrane windows enclosing fixed-hydrated cells and a buffer solution [33], which is described in section 4.3.1. These chambers, however, are closed containers, which do not allow for exchanging the liquid in the chamber after it is once assembled and therefore supplying the cells with nutrients or, in a further experimental step, manipulating the cells is not possible.

Microfluidic techniques provide an ideal tool for in situ manipulation of biological cells. However, the well-established microfluidic devices based on soft lithography 131, 132, which are often used in combination with optical microscopy and are fabricated of polymers like polydimethylsiloxane (PDMS), bring about the problem of showing a high X-ray absorption and a strong background signal in the small-angle region 133. A variety of X-ray compatible microfluidic devices based on different materials and fabrication techniques has been proposed in the past as reviewed by Köster and Pfohl [38]. In one example for a device design the channel geometry is defined by a thin film of a moldable polymer like PDMS and adhesive Kapton film is used as a window material [34, 35]. For these devices the adhesive layer can lead to problems in terms of background scattering or bioincompatibility depending on the specific experimental parameters. An interesting approach using Kapton as window material is also presented by Barrett et al. [36]. Here, the microfluidic devices are built of two Kapton films and the channels are written directly into the Kapton film using laser ablation.

Another example are microfluidic devices that are completely composed from the UV-curable Norland Optical Adhesive 81 (NOA 81) [37], which allows for molding sophisticated channel structures directly in NOA 81. Furthermore, briefly cured layers of NOA 81 can be attached to each other, thereby facilitating the fabrication of multilayered devices. However, severe radiation damage to this material restricts the use of this device type at high photon flux synchrotron beamlines. For our purpose of use, additional limitations in device design and material choice arise from specific requirements of living cells, since they need well-defined growth conditions like temperature or $\mathrm{pH}$ value, a continuous nutrient supply and also, in the case of adherent cells, a specific substrate. Since $\mathrm{Si}_{3} \mathrm{~N}_{4}$ membrane windows combine the properties of being a good substrate for cell growth with excellent X-ray scattering characteristics, meaning that they have a X-ray low absorption and produce no background scattering, we chose this material as a central part of the microfluidic device. To supply the cells with culture medium, we incorporated the $\mathrm{Si}_{3} \mathrm{~N}_{4}$ mem- 
(a)

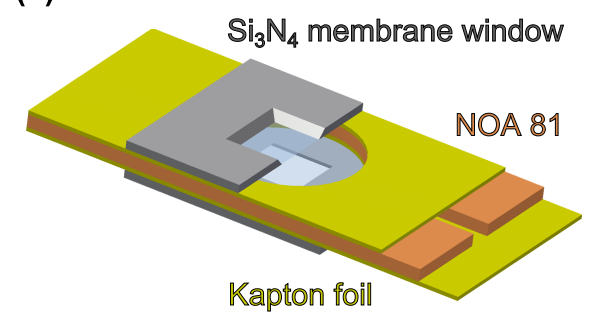

(b)

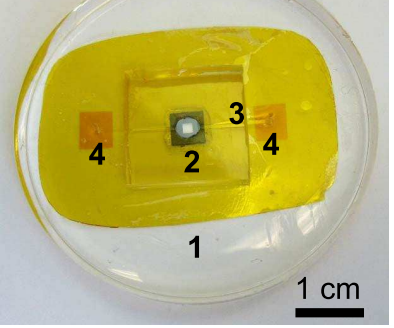

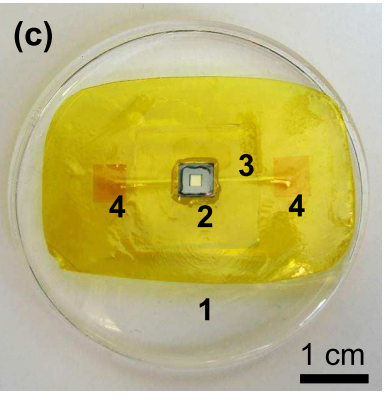

Fig. 4.8.: Three-dimensional schematic of a microfluidic device (a) and photographs of a microfluidic device with one $\mathrm{Si}_{3} \mathrm{~N}_{4}$ membrane window attached to the Kapton foil (b,c). (b) View on the inside of the chamber, in which the cells are grown and measurements are performed. (c) View on the outside of the device. (1) PDMS support with a hole in the center, (2) $\mathrm{Si}_{3} \mathrm{~N}_{4}$ membrane window, (3) channel, (4) inlet/outlet, which are sealed from one side with Kapton tape, for the connection of tubings and syringe pumps. [Figure adapted from [40.]

brane windows in a novel type of NOA 81-Kapton-device, as shown in Fig. 4.8. In these devices a microfluidic channel structure is laterally defined in a layer of NOA 81. The top and bottom of the channel are open in the NOA 81 layer and the channel is sealed by two thin Kapton foils without any additional adhesive. The fabrication of this device type is described in section 4.3 .2 .

\subsubsection{Sandwich Wet Chambers}

For first experiments on hydrated cell, sandwich wet chambers built of two $\mathrm{Si}_{3} \mathrm{~N}_{4}$ membrane windows [33] were used. As shown in Fig. 4.9 a the fixed-hydrated cells and a buffer solution were enclosed by two $\mathrm{Si}_{3} \mathrm{~N}_{4}$ membrane windows. To build these wet chambers, the cells were grown on $\mathrm{Si}_{3} \mathrm{~N}_{4}$ membrane windows and fixed as
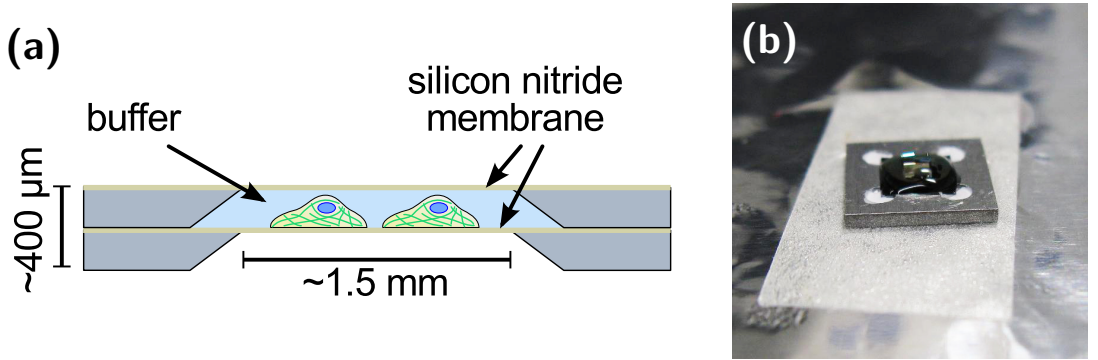

Fig. 4.9.: $\mathrm{Si}_{3} \mathrm{~N}_{4}$ sandwich wet chambers. (a) Sketch of a sandwich wet chamber built of two $\mathrm{Si}_{3} \mathrm{~N}_{4}$ membrane windows with cells and buffer solution between the membranes. (b) Photograph of one $\mathrm{Si}_{3} \mathrm{~N}_{4}$ membrane window being attached to an aluminum frame during incubation with BSA solution. 
described in section 4.2.1 and 4.2.3. A clean $\mathrm{Si}_{3} \mathrm{~N}_{4}$ membrane window was attached to an aluminum frame with hole in the center by applying nail polish to the corners of the silicon frame as shown in Fig. 4.9. The $\mathrm{Si}_{3} \mathrm{~N}_{4}$ membrane window was placed on the aluminum frame with the flat side facing downwards. About $20 \mu \mathrm{l}$ of $1 \%(\mathrm{w} / \mathrm{v})$ bovine serum albumin (BSA; Roth) solution were added on the upwards facing side of the $\mathrm{Si}_{3} \mathrm{~N}_{4}$ membrane window and incubated for $30 \mathrm{~min}$, which led to a coating of the surface with BSA and decreased dewetting of the surface during blotting. Afterwards the window was washed three times with the same buffer as used in the wet chamber.

The $\mathrm{Si}_{3} \mathrm{~N}_{4}$ membrane window with cells grown on the surface was taken out of the transport tube and the non-cell-side was blotted with filter paper. The window was place with the cell-side pointing downwards on the first window. The excess liquid was blotted from the edges of the frames until the silicon frames touched each other. The edges were sealed with a two component glue (UHU plus sofortfest, UHU, Bühl, Germany) and the glue was cured for about 15 min. Possibly remaining salt precipitates on the membrane were washed off with ultra-pure water.

\subsubsection{Fabrication of Microfluidic Devices}

The microfluidic devices were fabricated in a multi-step process, which is a further development of the process described by Brennich et al. [37]. In the following part, the fabrication of the devices and the final assembly of the devices at a beamtime are described.

\subsubsection{Photolithography}

A master for the device fabrication was produced by photolithography using SU-8 negative resist (SU-8 3050, MicroChem, Newton, MA, USA) as shown in Fig. 4.10a, b. The photolithography step was performed in a class 100 cleanroom to prevent impurities on the surfaces. The resist was spin-coated on a polished silicon wafer (SilChem, Freiberg, Germany) at a velocity of 800-1000 rpm yielding a layer thickness of about 130-215 $\mu \mathrm{m}$. After spin coating, a soft bake step was performed for $45 \mathrm{~min}$ at $95^{\circ} \mathrm{C}$. Subsequently the resist was exposed for $15 \mathrm{~s}$ to UV-light $(365 \mathrm{~nm}$, $22 \mathrm{~mW} / \mathrm{cm}^{2}$ ) through a photomask $\mathrm{I}^{3}$ using a mask aligner (MJB4, Süss MicroTec AG, Garching, Germany). As channel geometry, a straight channel with a width of $500 \mu \mathrm{m}$ or $700 \mu \mathrm{m}$ was chosen, but channel height, width and geometry can be easily adapted to specific requirements by choosing a different spinning speed, resist

\footnotetext{
${ }^{3}$ The photomask was designed with AutoCAD 2008, München, Germany, and it was produced by JD Photo-Tools, Oldham, UK.
} 
(a)

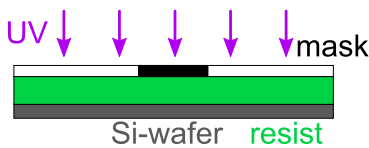

(b)

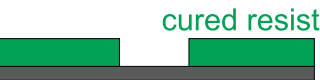

(c)

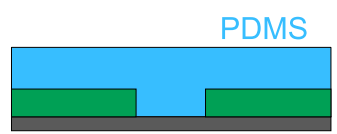

(d)

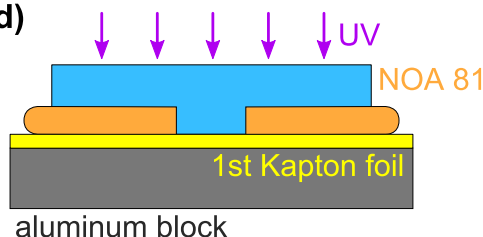

(e)

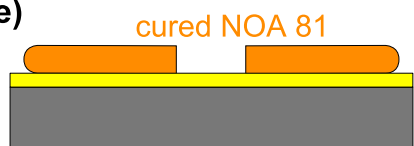

(f)

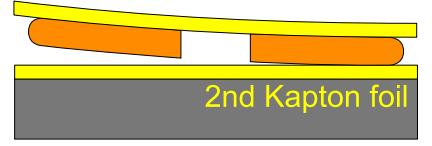

(g)

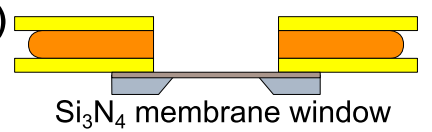

(h)

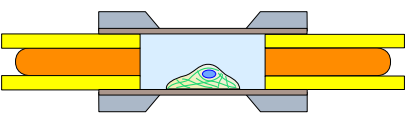

Fig. 4.10.: Schematic overview of the fabrication process of X-ray compatible microfluidic devices. (a) A thin layer of photoresist was spin-coated on a silicon wafer and exposed to UV-light through a photomask. (b) After development of the resist, previously nonexposed regions become channels in the resist. (c) A PDMS stamp was cast from the wafer yielding a negative of the resist structure. (d), (e) Kapton foil with a thickness of $8 \mu \mathrm{m}$ was attached to a flat and polished aluminum block and about $100 \mu \mathrm{l}$ NOA 81 were pipetted on the Kapton foil. The PDMS stamp was placed on the NOA 81 and pushed down until the channel touched the Kapton foil. NOA 81 was cured by exposure to UV-light and the PDMS stamp was removed. (f) A second Kapton foil was attached to another aluminum block. The Kapton foil with the NOA 81 film was removed from the aluminum block and carefully placed on the second Kapton foil. (g) A hole was punched through the device in the center of the channel and one side was sealed with a $\mathrm{Si}_{3} \mathrm{~N}_{4}$ membrane window. (h) Cells were grown on the window and, after cell fixation or after they are attached, the device was closed with a second $\mathrm{Si}_{3} \mathrm{~N}_{4}$ membrane window. [Figure adapted from [40].]

or photomask. After exposure, the wafer was baked for $1 \mathrm{~min}$ at $65^{\circ} \mathrm{C}$ and $15 \mathrm{~min}$ at $95^{\circ} \mathrm{C}$. Finally, the structure was developed for about $20 \mathrm{~min}$ in SU-8 developer (MicroChem). The wafer with the cured resist, as shown in Fig. 4.10p, was vapor-coated with (heptafluoropropyl)-trimethylsilane (Aldrich, Steinheim, Germany), which enables a smooth removal of the PDMS stamp produced in the next step.

\subsubsection{Kapton-NOA 81 Device with one $\mathrm{Si}_{3} \mathrm{~N}_{4}$ Membrane Window}

PDMS and cross-linker (Sylgard 184, Dow Corning, Midland, MI, USA) were mixed at a ratio of $10: 1$, degassed and poured on the silicon wafer (see Fig. 4.10c). The PDMS was degassed again until all gas bubbles were removed and baked for $2 \mathrm{~h}$ at $65^{\circ} \mathrm{C}$. After baking, the PDMS stamp was removed from the wafer. For the device fabrication, stamps with a channel cross section (width $\times$ height) of $500 \times 132 \mu \mathrm{m}^{2}$, $700 \times 167 \mu \mathrm{m}^{2}$ and $700 \times 214 \mu \mathrm{m}^{2}$ were used.

For molding of the channel in NOA 81, a circular piece of $8 \mu \mathrm{m}$ thick Kapton foil (SPEX SamplePrep, Metuchen, NJ, USA) was attached via capillary forces to a flat and polished aluminum block by applying a small drop of isopropanol between 
the Kapton foil and the aluminum block. Wrinkles in the foil were removed with a tissue and excess isopropanol was squeezed out, until the Kapton foil was tightly attached to the aluminum block. $100 \mu \mathrm{l}$ NOA 81 (Norland, Cranberry, NJ, USA) were pipetted onto the Kapton foil (see Fig. 4.11 ) and onto the structure on the PDMS stamp and air bubbles were removed with a small pipette tip.

The stamp was placed on the Kapton foil and pushed down until the elevated channel on the PDMS stamp touched the Kapton foil as shown in Fig. 4.10d and Fig. 4.11 b. This assures that the channel was only laterally defined by NOA 81, while the top and bottom were open in the NOA 81 layer. NOA 81 was cured through the PDMS stamp by exposure to UV-light for $2 \mathrm{~min}$ ( $8 \mathrm{~W}$ at $365 \mathrm{~nm}$ ). The curing through the PDMS stamp leaves a thin layer of NOA 81 at the NOA 81/PDMS
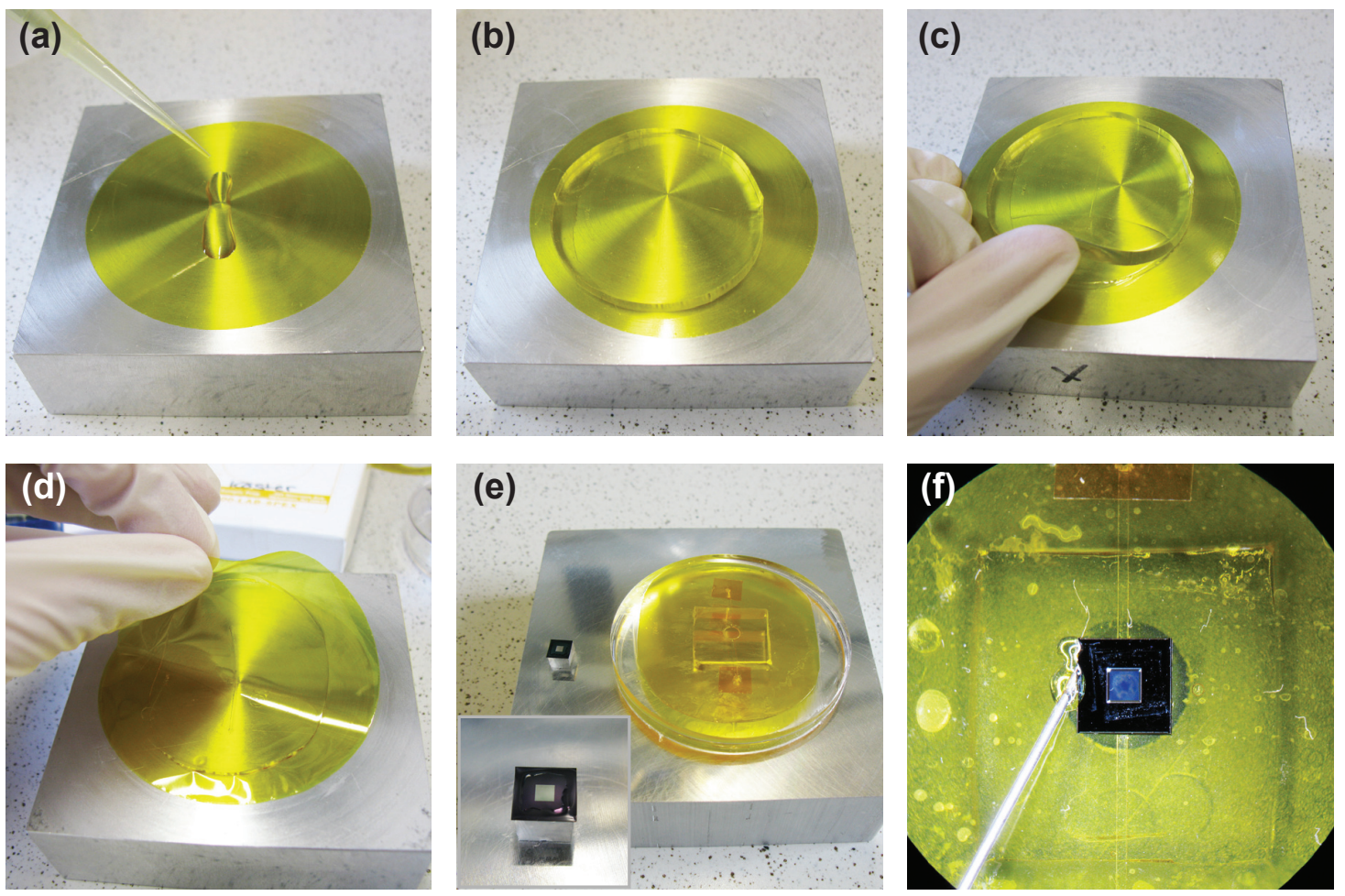

Fig. 4.11.: Photographs of the device fabrication process. (a) The Kapton foil was attached to a flat aluminum block and NOA 81 was pipetted onto the foil. (b) The PDMS stamp was put onto the Kapton foil and pushed down until the elevated channel touched the Kapton foil. (c) After curing of the NOA 81, the PDMS stamp was removed and (d) the NOA 81 film with the Kapton foil was attached to a second Kapton foil. (e) A hole was punched through the center of the device and a PDMS support was attached to it. The $\mathrm{Si}_{3} \mathrm{~N}_{4}$ membrane window was placed on a small block of PDMS and Loctite was applied to the outermost edges of the frame. (f) After gluing the $\mathrm{Si}_{3} \mathrm{~N}_{4}$ membrane window with Loctite to the device, the edges of the frame are sealed with a two component glue with a needle. 
interface uncured 37, 134, 135 and therefore allows for sealing the device with a second layer of Kapton foil with no need for an additional glue or adhesive.

After curing of the NOA 81, the PDMS stamp was carefully removed leaving the cured NOA 81 film on the Kapton foil (see Fig. 4.10 and Fig. 4.11k) and a second Kapton foil was attached to another aluminum block as described above. The Kapton foil with the NOA 81 film was removed from the aluminum block and attached with the NOA 81 side to the second Kapton foil as shown in Fig. 4.10f and Fig. 4.11 d. At this stage of device fabrication, the channel was defined in lateral direction by NOA81 and the top and bottom regions are sealed with Kapton. To incorporate $\mathrm{Si}_{3} \mathrm{~N}_{4}$ membrane windows in the device, a hole is punched through the device in the center of the channel using a Harris Uni-CoreTM puncher (Plano, Wetzlar, Germany) with a diameter of $3.5 \mathrm{~mm}$.

For connection to a syringe system the device is first glued with Loctite polyolefin (Loctite 770 and 406, Henkel, Düsseldorf, Germany) to a flat, circular PDMS piece with a hole in the center. Afterwards holes for the inlets and outlets are punched through the Kapton-NOA81 devices and the PDMS and the holes on the Kaptonside of the devices are closed with adhesive Kapton tape (Dr. D. Müller GmbH, Ahlhorn, Germany). Fig. 4.11 shows a photograph of the device at this fabrication stage.

The $3.5 \mathrm{~mm}$ diameter hole in the center device is closed from one side with a $\mathrm{Si}_{3} \mathrm{~N}_{4}$ membrane window. For this purpose, the $\mathrm{Si}_{3} \mathrm{~N}_{4}$ membrane window is first placed on a small PDMS block with the flat side facing upwards as shown in Fig. 4.11k. The frame was held on the PDMS block via capillary forces. Loctite polyolefin primer 770 was applied locally around the hole in the device at the non-PDMS side and Loctite 406 (Henkel) was applied with a small pipette tip to the outermost part of the frame of the $\mathrm{Si}_{3} \mathrm{~N}_{4}$ membrane window. The hole in the device was positioned on the $\mathrm{Si}_{3} \mathrm{~N}_{4}$ membrane window using a stereoscopic microscope and the PDMS block was subsequently removed (see Fig. $4.10 \mathrm{f}$ ). Additionally, the edges of the $\mathrm{Si}_{3} \mathrm{~N}_{4}$ membrane window were sealed with a two-component glue (UHU plus schnellfest) using an injection needle as shown in Fig. 4.11f. Photographs of the device from both sides at this stage of the fabrication process are shown in Fig. $4.8 \mathrm{~b}, \mathrm{c}$.

\subsubsection{Final Device Assembly}

To obtain a sample chamber, as shown in Fig. 4.10f and in the three-dimensional schematic in Fig. $4.8 \mathrm{a}$, a second $\mathrm{Si}_{3} \mathrm{~N}_{4}$ membrane window was attached to the device directly before the measurement during a beamtime. For the experiments on hydrated cells in microfluidic devices, the cells can be either grown on the first $\mathrm{Si}_{3} \mathrm{~N}_{4}$ membrane window while the sample chamber is still open or on the second 

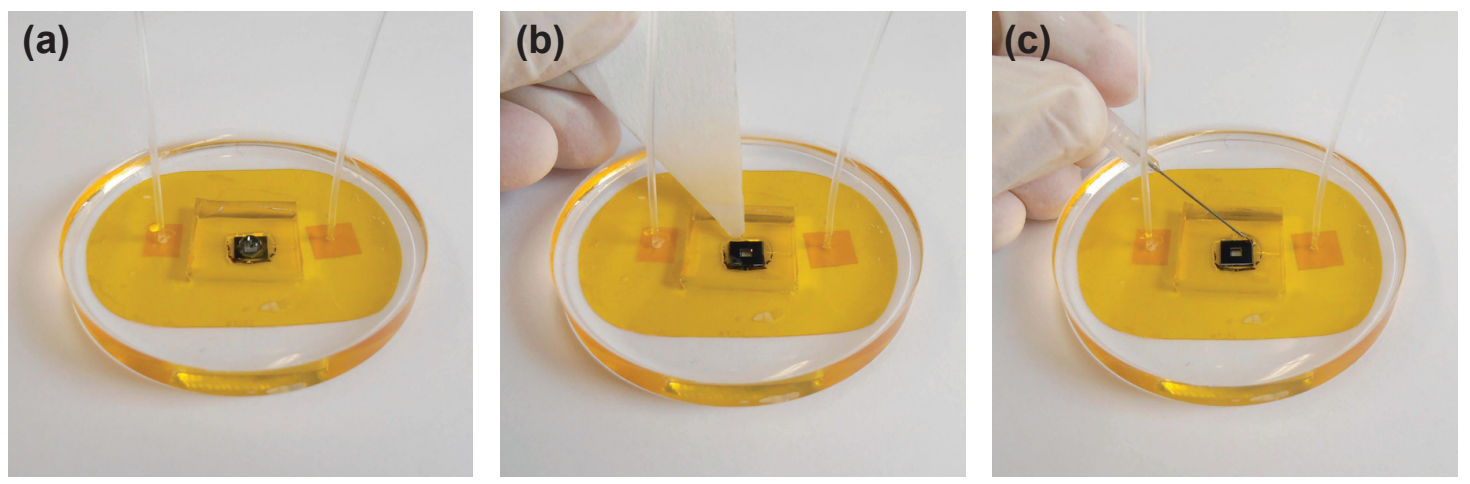

Fig. 4.12.: Photographs of the devices assembly process. (a) Syringes were attached to the inlet and outlet and the channels were flushed with liquid until the $\mathrm{Si}_{3} \mathrm{~N}_{4}$ membrane window was covered with a small drop. (b) The second $\mathrm{Si}_{3} \mathrm{~N}_{4}$ membrane window was put onto the first one and excess liquid was blotted with a filter paper. (c) The edges of the window were sealed with a two-component glue.

window that is used for closing the sample chamber. Both methods circumvent the problems of bringing adherent cells into closed channels and supplying the not-yet adhered cells with nutrients without washing them out of the channels. For first experiments, the cell were grown on the $\mathrm{Si}_{3} \mathrm{~N}_{4}$ membrane window that was attached to the device [40]. Later, the cells were grown on the second $\mathrm{Si}_{3} \mathrm{~N}_{4}$ membrane window, because this allowed to combine the best microfluidic devices with the most promising cells.

For the device assembly, a $2.5 \mathrm{ml}$ Hamilton Gastight glass syringe (Bonaduz, Switzerland) and a disposable syringe both filled with degassed buffer or $\mathrm{CO}_{2}$ independent medium (PAA Laboratories GmbH) supplemented with 10\% FCS, $100 \mathrm{U} / \mathrm{ml}$ penicillin and $0.1 \mathrm{mg} / \mathrm{ml}$ streptomycin were attached to the inlet and outlet, respectively, via polyethylene tubings (inner diameter $0.38 \mathrm{~mm}$, outer diameter $1.09 \mathrm{~mm}$, Intramedic Clay Adams Brand, Becton Dickinson and Company, Sparks, Maryland, USA). For attachment of the second window, the channels were flushed with liquid until the $\mathrm{Si}_{3} \mathrm{~N}_{4}$ membrane window was covered with a small drop (see Fig. 4.12 a). The $\mathrm{Si}_{3} \mathrm{~N}_{4}$ membrane window with the cells was taken out of a transport tube and the liquid on the hole-side of the window and in between the two arms of the tweezers was blotted. Note that the cells were always grown on the flat side of the membrane. The window was placed with the flat side pointing downwards on the liquid drop on the first window. The excess liquid was blotted with a filter paper until the second window touched the Kapton foil as shown in Fig. $4.12 \mathrm{~b}$. The window was fixed and the edges were sealed with a two-component glue (UHU plus sofortfest), which was applied with a needle as displayed in Fig. 4.12c. After about $15 \mathrm{~min}$ the glue was cured and the disposable syringe at the outlet was removed. Possibly remaining salt 
precipitates on the membrane were washed off with ultra-pure water.

Degassing of the buffer or medium is a crucial step for X-ray experiments, because otherwise the exposure to X-rays leads to the formation of gas bubbles in the device. Best degassing was obtained by using a filtration unit (Sartorius, Göttingen, Germany) that was connected to a membrane pump and placed in an ultrasonic bath. The buffer was degassed with the filtration unit and sonicated for about $20 \mathrm{~min}$. 


\section{Scanning Nano-Diffraction on Freeze-Dried Cells}

In this chapter, scanning X-ray nano-diffraction experiments on freeze-dried SK8/182 cells are described. Different steps of the data analysis are presented using the example of data sets that were recorded at the ID13 beamline. The results obtained from measurements at three different synchrotron radiation facilities are presented and discussed, and the capabilities of the different set-ups are compared. Parts of this chapter have been published in New Journal of Physics [39].

\subsection{Experiment}

The experiments were carried out at three different end-stations at different synchrotron radiation facilities: the cSAXS beamline (SLS), the ID13 beamline (ESRF), and the P10 beamline (PETRA III). The different setups and the specific experimental parameters during each beamtime are described separately in section 3.2 . In the following, a reference to the set-up or beamtime at which a certain data set was recorded will be given as the name of the beamline and the date (month and year) of the beamtime, e.g., "P10, Oct. 2012".

The measured freeze-dried samples can be grouped in three different categories: cells without a keratin network, cells with a keratin network and cells (with a keratin network) that were treated with the IF lysis buffer. All freeze-dried cells were grown on $\mathrm{Si}_{3} \mathrm{~N}_{4}$ membrane windows, plunge-frozen and freeze-dried as described in section 4.2.4. The membrane windows were mounted on sample holders ("crocodile holders") as shown in Fig. 5.1 and the sample holders were mounted on the sample stage at the beamlines. The samples

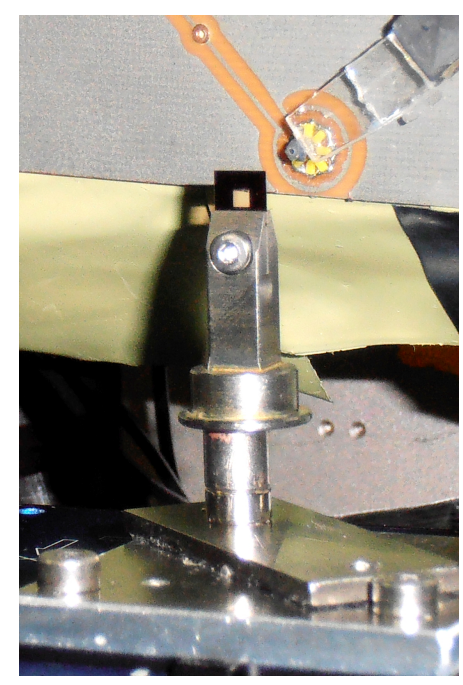

Fig. 5.1.: $\quad \mathrm{Si}_{3} \mathrm{~N}_{4}$ membrane window mounted on a crocodile sample holder (ID13, Nov. 2011). were aligned in the focus of the X-ray beam using a 
(a)

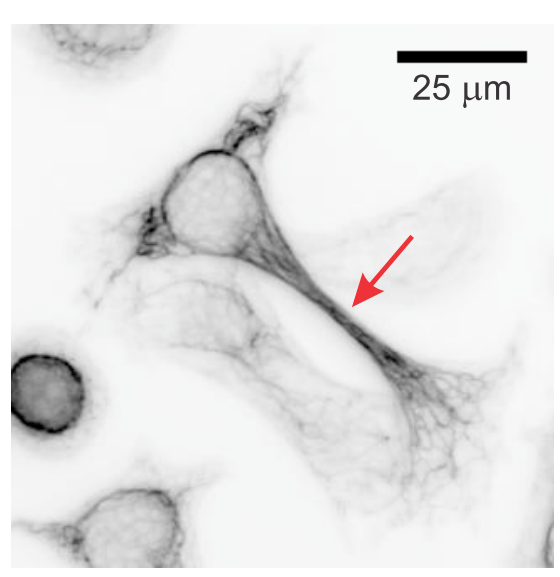

(b)

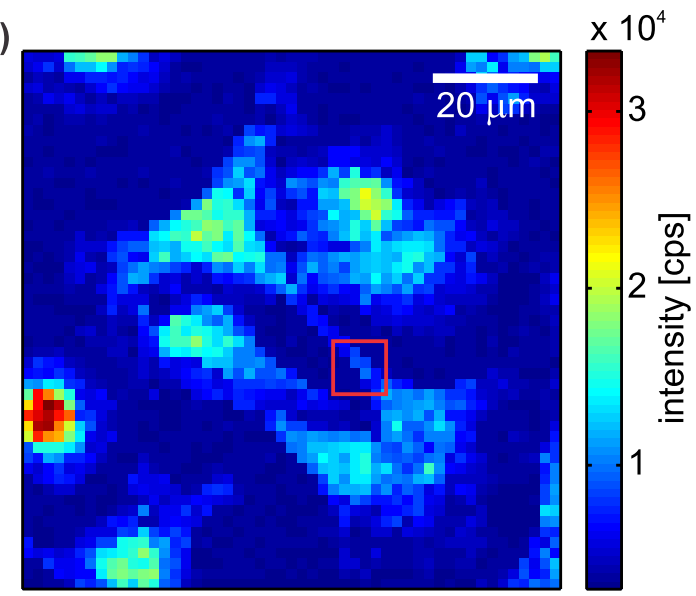

(c)

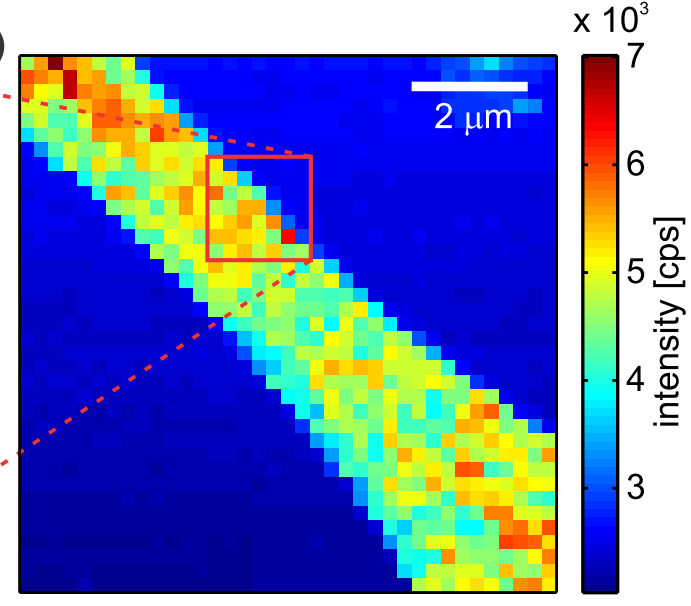

7

(1)

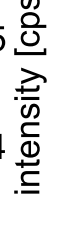

(d)

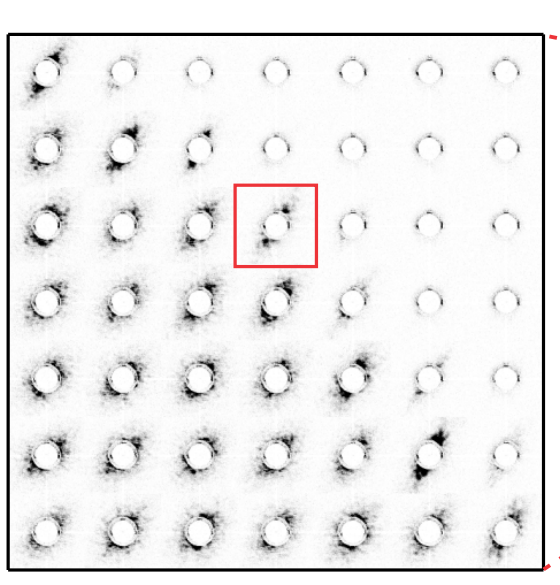

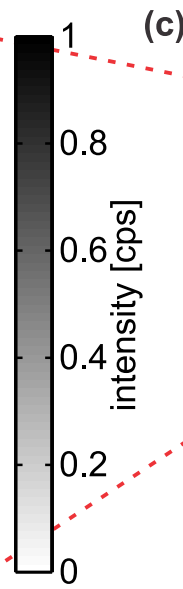

Fig. 5.2.: X-ray diffraction from highly aligned keratin bundles in freeze-dried cells. (a) Inverted fluorescence microscopy image of the keratin network in SK8/18-2 cells. The image was taken on formaldehyde-fixed cells, before plunge-freezing and freeze-drying. (b) X-ray dark-field image from a coarse scan on a large sample area with a step size of $2 \mu \mathrm{m}$ and an exposure time of $0.1 \mathrm{~s}$. (c) X-ray dark-field image from a region of interest scan recorded on the keratin-rich extension of one cell with a step size of $250 \mathrm{~nm}$ and an exposure time of $10 \mathrm{~s}$. (d) Composite image of the scattering patterns corresponding to the marked region in (c) showing a strong degree of orientation in the scattering signal. The scattering pattern marked by a red square is used as an example for the analysis of a single scattering pattern later on (Sample BW17, position 1; ID13, June 2011) [Figure adapted from [39.]

calibrated visible light microscope. Two-dimensional mesh scans were performed using piezoelectric motors and scattering patterns were recorded on a two-dimensional detector at each scan position. Depending on the total flux at the beamline, the exposure time and the dynamic range of the detector, the primary beam was blocked with a beamstop to prevent saturation of the detector.

To check the sample alignment in the X-ray beam, coarse mesh scans as shown in 
Fig. $5.2 \mathrm{~b}$ with a step size of $1-2 \mu \mathrm{m}$ and a short exposure time were performed upon changing the sample or moving to a different position. Real space images of the samples were calculated using X-ray dark-field or differential phase contrast and compared to the visible light microscopy images that had been taken beforehand. After verification of sample positioning, finer mesh scans with step sizes in the order of the beam size, i.e. 100-400 nm, and longer exposure times as shown in Fig. $5.2 \mathrm{c}$ and $\mathrm{d}$ were performed on small sample regions. Here, we particularly selected cells with a distinct keratin network morphology as identified from the fluorescence microscopy images as shown in 5.2 a. This allowed for a potential correlation of structural information obtained from X-ray diffraction experiments with the fluorescence microscopy images of the keratin network. Due to the high photon flux at the P10 beamline, the samples were already visibly destroyed after a first scan with a short exposure time in the order of $0.05 \mathrm{~s}$. Therefore fine region of interest scans were not performed at this set-up. Instead, also smaller step sizes of $500 \mathrm{~nm}$ were employed for large area scans. 


\subsection{Results from Measurements at ID13 Beamline}

At the ID13 beamline, experiments were performed on freeze-dried cells with a keratin network, keratin-free cells and cells (with a keratin network) that were treated with the IF lysis buffer. An overview of the measurements presented here on freezedried cells taken at the ID13 beamline along with the employed scan parameters and the estimated average radiation doses per step is shown in Tab. 5.1.

In the following section, different steps of the data analysis are described using the example of measurements on a cell with a keratin network. Afterwards the same analysis scheme is applied to a keratin-free cell and a cell that was treated with the IF lysis buffer, then the results of the different sample types are compared.

\begin{tabular}{llllllll}
\hline sample & set-up & \multicolumn{2}{c}{$\Delta_{y, z}\left[\mu \mathrm{m}^{2}\right]$} & $N_{y} \times$ & $N_{z}$ & $T[\mathrm{~s}]$ & $D[\mathrm{~Gy}]$ \\
\hline BW17, pos. 1A & June 2011 & 2 & $\times 2$ & $51 \times 51$ & 0.1 & $2.7 \times 10^{4}$ \\
BW17, pos. 1B & June 2011 & $0.25 \times 0.25$ & $37 \times 37$ & 10 & $1.7 \times 10^{8}$ \\
BW21, pos. 4A & Nov. 2011 & 1 & $\times 1$ & $81 \times 71$ & 0.1 & $1.1 \times 10^{7}$ \\
BW21, pos. 4B & Nov. 2011 & $0.1 \times 0.1$ & $101 \times 101$ & 1 & $1.1 \times 10^{8}$ \\
BW21, pos. 6A & Nov. 2011 & 2 & $\times 2$ & $41 \times 31$ & 0.1 & $2.7 \times 10^{4}$ \\
BW21, pos. 6B & Nov. 2011 & $0.1 \times 0.1$ & $61 \times 61$ & 1 & $1.1 \times 10^{8}$ \\
BW21, pos. 6C & Nov. 2011 & $0.25 \times 0.25$ & $41 \times 37$ & 10 & $1.7 \times 10^{8}$ \\
BW54, pos. 1A & June 2011 & 2 & $\times 2$ & $56 \times 56$ & 0.1 & $2.7 \times 10^{4}$ \\
BW54, pos. 1B & June 2011 & $0.1 \times 0.1$ & $41 \times 41$ & 1 & $1.1 \times 10^{8}$ \\
\hline
\end{tabular}

Tab. 5.1.: Summary of the scan parameters and the estimated average radiation doses per step for the measurements on freeze-dried SK8/18-2 cells at the ID13 beamline. Here, $\Delta_{y, z}$ are the lateral step sizes, $N_{y}$ and $N_{z}$ are the number of scan points in $y$ - and $z$ direction, $T$ is the exposure time and $D$ the estimated average radiation dose per step. All measurements were performed without attenuators in the beam path.

\subsubsection{Cells with a Keratin Network}

\subsubsection{X-Ray Dark-Field Images and Structure Orientation}

One interesting example of a cell exhibiting a pronounced KIF network morphology is the cell in the center of Fig. 5.2 a exhibiting a long extension (red arrow), which contains several thick keratin bundles. In the corresponding X-ray dark-field image in Fig. $5.2 \mathrm{~b}$, which was reconstructed from a coarse mesh scan with a step size of $2 \mu \mathrm{m}$ and an exposure time of $0.1 \mathrm{~s}$, a high contrast between cellular material and 
substrate is visible, even though biological samples are generally weak scatterers. In particular, the nuclei of the imaged cells show a high scattered intensity, but also the periphery and the cellular extension pointed out in Fig. $5.2 \mathrm{a}$ can be clearly identified.

On the keratin-rich extension, a region of interest scan with a smaller step size of $250 \mathrm{~nm}$ and a longer exposure time of $10 \mathrm{~s}$ was performed. Again, the dark-field image in Fig. 5.2c shows a high contrast between the cellular extension and the substrate. Further, the integrated intensity is not homogeneous over the whole extension, but varies from scan point to scan point indicating a substructure in the sample. When looking at individual scattering patterns, shown as a composite image for a small region of this scan in Fig. $5.2 \mathrm{~d}$, highly anisotropic scattering signals are visible, which indicate an oriented substructure in the sample. Therefore it is not only possible to distinguish between cellular material and empty regions, as shown by the dark-field images, but the X-ray scattering patterns in reciprocal space can also be used to obtain information about the local sample structure.

Individual scattering patterns from the sample exhibit highly anisotropic signals, as can be seen from the raw data scattering pattern in Fig. $5.3 \mathrm{a}$. The anisotropy of the scattering signal, and accordingly the anisotropy in the sample structure, provides additional information to the X-ray dark-field contrast images. To capture the signal anisotropy the overall degree and direction of signal orientation in each scattering pattern were determined by approximating the thresholded intensity distribution by an ellipse. For this analysis, the raw data images were processed by first masking dead or hot pixels and pseudo pixels without sensitivity as shown in Fig. $5.3 \mathrm{~b}$. Subsequently the images were filtered with a $3 \times 3$ median filter to reduce the noise and thresholded with a threshold value of $0.1 \mathrm{cps}$ resulting in images as shown in Fig. 5.3c and d, respectively. Therefore, all pixel with photon counts above the threshold value are weighted equally in the binary image, meaning that the weight of the weak scattering at high $q$-values is increase compared to the stronger scattering at small $q$-values. To be able to treat scattering patterns from empty regions, which show nearly no intensity in the thresholded scattering patterns, with the same method, a circular intensity distribution was added in the central region of the scattering pattern where the intensity was blocked by the beamstop (see Fig. 5.3. $)$. This assured that scattering patterns from empty regions yielded an approximation by a circle, which corresponds to isotropy in the scattering signal. The thresholded intensity distribution was then approximated by an ellipse, which had the same second moments as the intensity distribution. Fig. 5.3: shows a thresholded scattering pattern along with the approximated ellipse and the principle axes. From the fitted ellipse, 
(a)

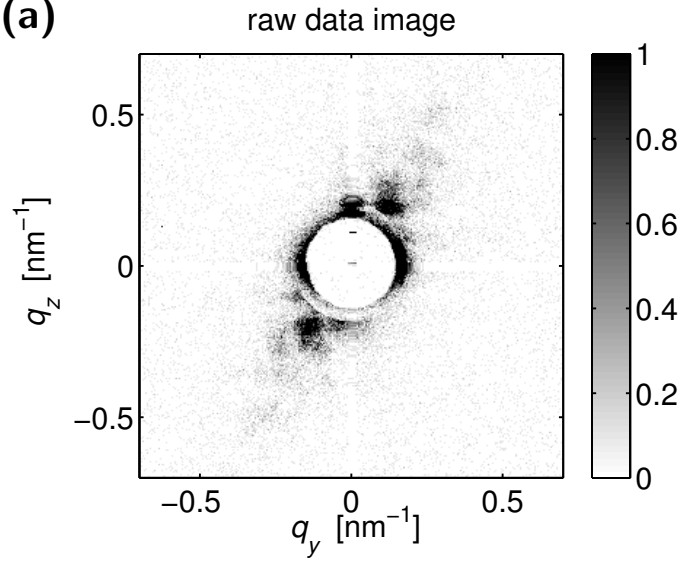

(c)

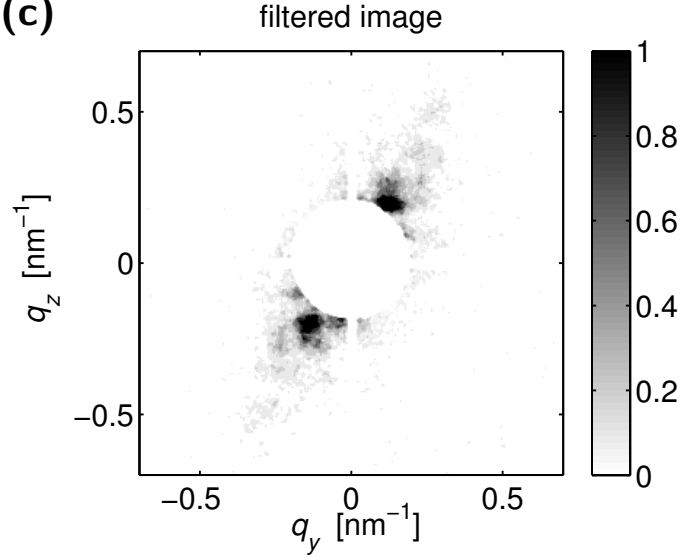

(e) binary image with circle in the center

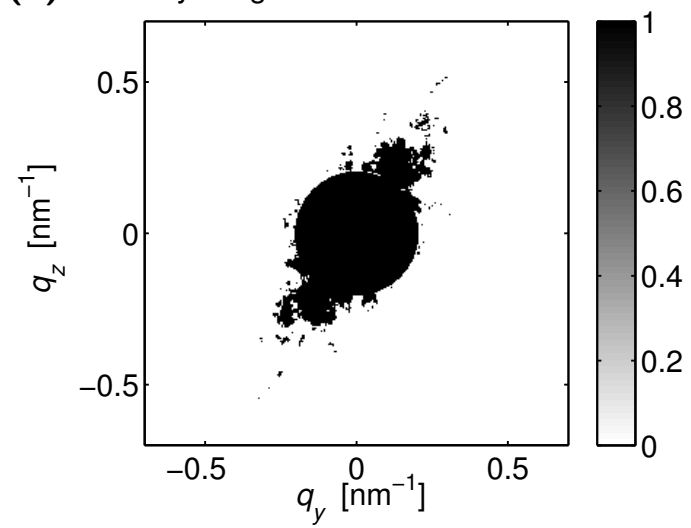

(b) raw data image multiplied with pixel mask

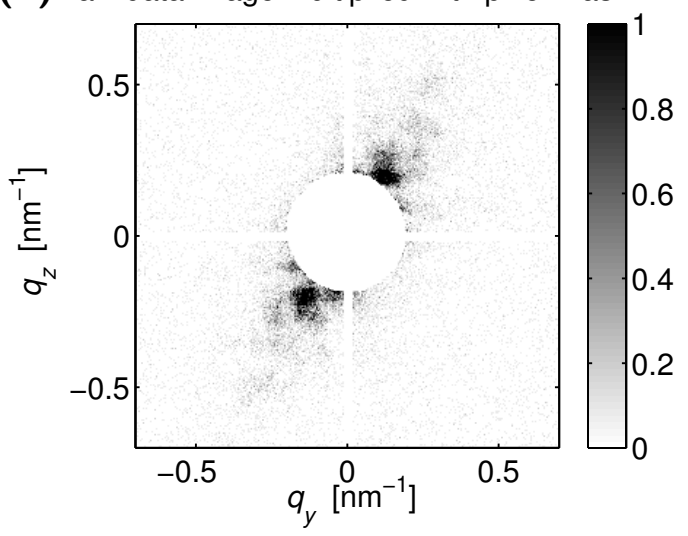

(d)

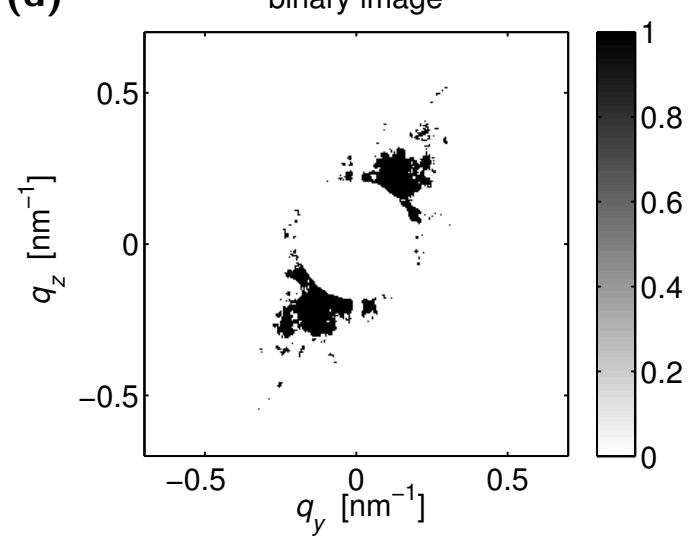

(f) binary image with fitted ellipse

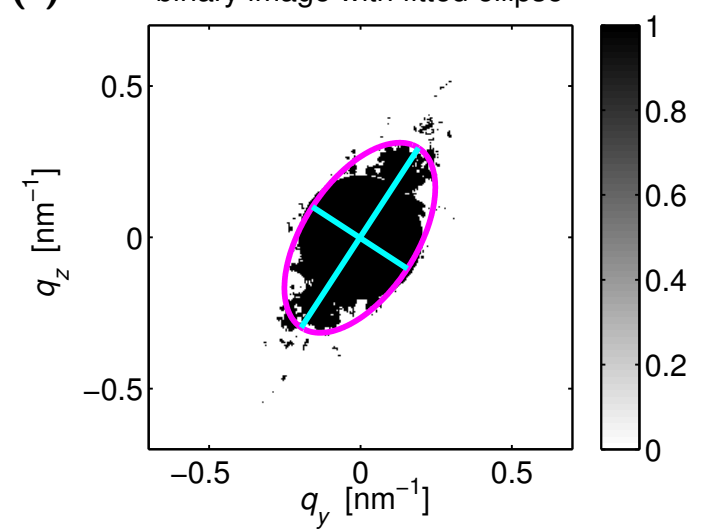

Fig. 5.3.: Image processing for the determination of the signal anisotropy. (a) Raw data image as recorded with a Maxipix detector. (b) Scattering pattern after multiplication with a pixel mask for selection of the valid pixels. (c) Scattering pattern after noise reduction by filtering with a $3 \times 3$ median filter. (d) Thresholded scattering pattern. (e) Thresholded scattering pattern with circle in the center. (f) Thresholded scattering pattern together with a fitted ellipse (magenta), which has the same second moments as the intensity distribution. The major and minor axes of the ellipse are shown in cyan. (Sample BW17, position 1B; ID13, June 2011) 
the direction of the minor axis in reciprocal space and the eccentricity ${ }^{1}$ were determined. The direction of the minor axis indicates the average direction of oriented structures in the sample in real space at the measured position and the eccentricity presents a measure for the average degree of orientation.

Applying this analysis to each scattering pattern of a scan yielded two-dimensional maps of the degree and direction of the structure orientation in the sample as shown in Fig. 5.4 a and b. On the cellular extension the eccentricity is high and the scattering signal points in roughly the same direction. On the empty region the eccentricity is very small and the orientation of the scattering signal is random. Together, the degree and direction of orientation in the sample can be visualized as a vector field, where the arrow length and direction are determined by the eccentricity and the direction of the minor axis of the ellipse, respectively. An overlay of the vector field, where the arrowheads of the vectors are omitted due to no physical relevance in this analysis, along with the corresponding dark-field image for the scan on the cellular extension is shown in Fig. 5.4k. The vector field indicates structures in the sample that are oriented parallel to the direction of the cellular extension, which is in-line with the fluorescence microscopy images of the keratin network in Fig. 5.2 a showing densely packed keratin bundles in the cellular extension.

The same analysis was applied to a mesh scan with a step size of $100 \mathrm{~nm}$ covering an area of $10 \times 10 \mu \mathrm{m}^{2}$ on a different cell of the same type. The large overview scan on this cell is shown in Fig. 5.5; along with the visible light fluorescence microscopy image of the keratin network in the hydrated cell before plunge-freezing and freeze-drying and the phase contrast microscopy image of the freeze-dried cell in Fig. 5.5a, b. The red box in Fig. 5.5c marks the position of the finer region of interest scan. An overlay of the corresponding dark-field image and the orientation map from the region of interest scan is presented in Fig. 5.5d. The dark-field image shows a network-like intensity distribution with an apparent feature thickness of about 200-400 nm, which is close to the resolution limit due to the used beam size. The local orientation, determined from the scattering signal anisotropy, agrees very well with the direction of the elongated structures in the dark-field image. The feature thickness in the X-ray dark-field image along with the structure orientation indicate that the observed structures might correspond to the keratin network in the sample. Differences between the X-ray dark-field image in combination with the orientation map, and the fluorescence microscopy image might be attributed to different factors. The fluorescence microscopy images have comparatively low resolution and therefore thin keratin bundles are invisible. Additionally, only the keratin network is visualized in the fluorescence microscopy images, but other cy-

\footnotetext{
${ }^{1}$ The eccentricity is defined as $\epsilon=\sqrt{1}-a^{2} / b^{2}$ with the minor and major axis $a$ and $b$.
} 
toskeletal structure that are not shown in the fluorescence microscopy image, like microtubules or actin filaments, might cause a similar scattering signal. The fluorescence microscopy images are taken on hydrated samples in buffer, whereas the

(a)

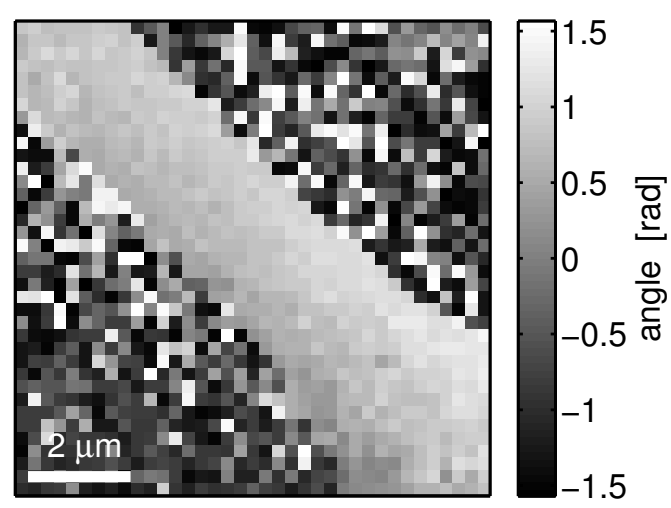

(b)

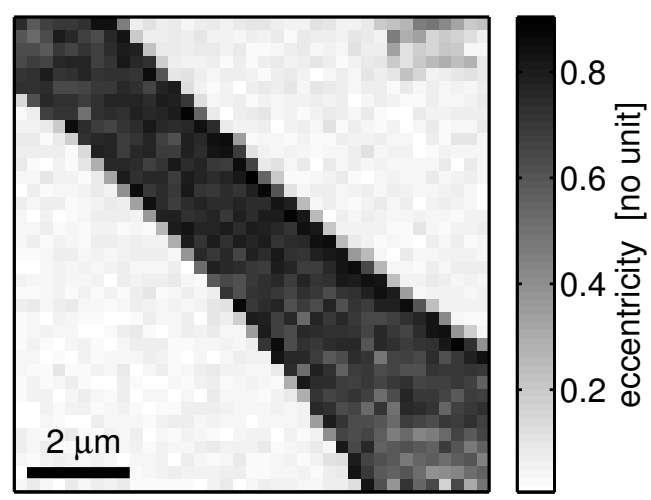

(c)

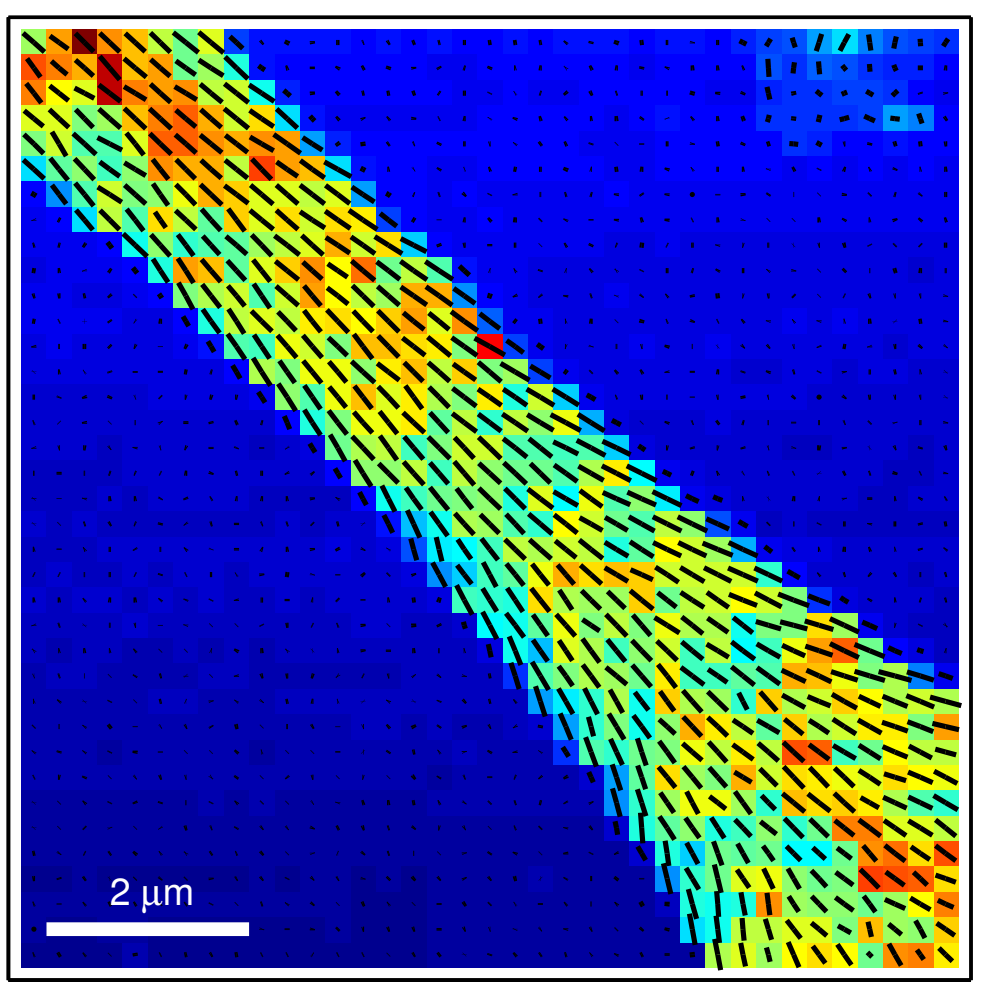

7000

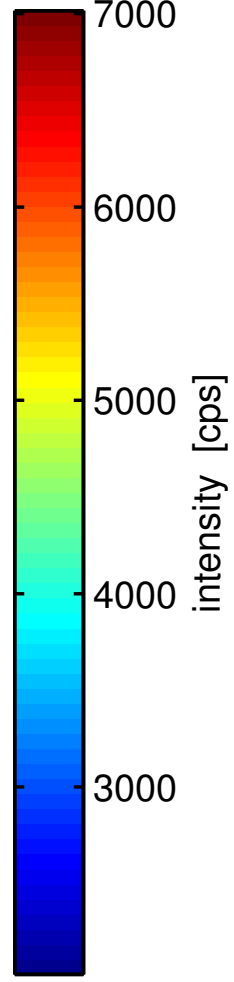

Fig. 5.4.: Structure orientation in the cellular extension of a freeze-dried SK8/18-2 containing a keratin network. (a) Direction of structure orientation indicated by the orientation of the minor axis of the ellipse and (b) degree of structure orientation indicated by eccentricity of the ellipse at each position of the scan. (c) The orientation of the minor axis and the eccentricity of the ellipse are plotted as a vector field (arrowheads are omitted, due to no physical relevance) in overlay with the corresponding dark-field image. (Sample BW17, position 1B; ID13, June 2011) 

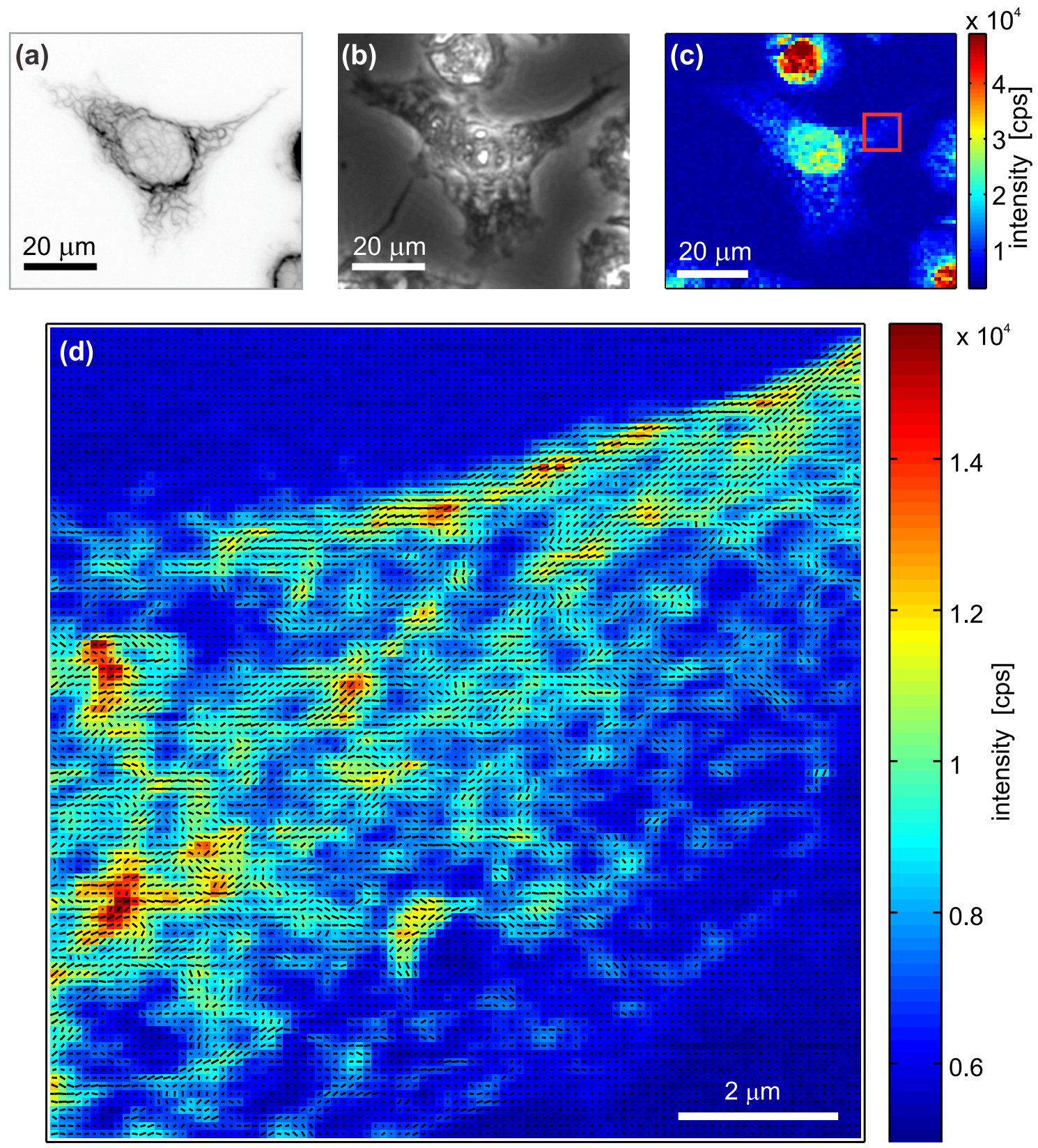

Fig. 5.5.: Structure orientation map and dark-field image of the cell body of a freeze-dried SK8/18-2 cell. (a) Fluorescence microscopy image of the keratin network in a hydrated SK8/18-2 cell before plunge-freezing and freeze-drying and (b) visible light phase contrast microscopy image of the same cell after plunge-freezing and freeze-drying. (c) X-ray darkfield image reconstructed from a coarse mesh scan with a step size of $1 \mu \mathrm{m}$ and an exposure time of $0.1 \mathrm{~s}$ on the same cell as in $(\mathrm{a}, \mathrm{b})$. (d) Structure orientation map as overlay with the dark-field image of a region on the cell body of a freeze-dried SK8/18-2 cell reconstructed from a mesh scan with a step size of $100 \mathrm{~nm}$ and $1 \mathrm{~s}$ exposure time. The scanned region is marked by a red box in panel (c). (Sample BW21, position 4; ID13, Nov. 2011) 
diffraction experiments are performed on freeze-dried samples and artifacts from the preparation cannot be excluded. In particular, protein or salt accumulations might be generated during plunge-freezing in case of the formation of (expanding) ice crystals instead of vitrified ice, which could lead to network-like structures in the $\mathrm{X}$-ray dark-field image.

\subsubsection{Radial Intensities of Averaged and Single Scattering Patterns}

As described above, at each scan point a scattering pattern in reciprocal space was recorded during the measurement. The scattering signal was analyzed by azimuthal integration of single and averaged scattering patterns. To account for possibly present anisotropy in the scattering signal, the reciprocal space was divided into eight angular segments in such a way that the first and the fifth segment were centered symmetrically around the major axis of the ellipse approximating the scattering signal. Average scattering patterns recorded on the cellular extension and on the empty region are shown in Fig. 5.6a, b and the angular segments are indicated by dashed, white lines. Here, the same position of the angular segments was used for the azimuthal integration of the average scattering pattern from the empty region and the cell region. The insets indicate from which regions of the scan the scattering patterns were used to obtain an average scattering pattern from the cellular extension and the empty region: Only scattering patterns recorded at the black positions were used for averaging. Azimuthal integration of the average scattering pattern from cell and background regions yielded the radial intensities shown in Fig. 5.6c and segment-wise background subtraction resulted in the radial intensity profiles shown in Fig. 5.6d. As expected intuitively for the case of an ori-

ented averaged scattering pattern, the radial intensity profiles in Fig. 5.6d separate in three groups: the intensity profiles in direction of the major and minor axis of the ellipse show highest and lowest scattering intensity, respectively, and the intensity profiles in-between the principle axes show intermediate scattering intensity.

All radial intensity profiles can be well described by a power law decay of the form $f\left(q_{r}\right)=a q_{r}^{b}+c$, as shown in Fig. 5.7 a exemplarily for one segment. The power law exponents obtained from the fits range from -4.00 to -4.25 as shown in Fig. $5.7 \mathrm{~b}$ with a mean value of -4.15 . Here, leaving out data points in the lowest $q_{r}$-region for fitting, slightly changed the power law exponents, which stayed, however, all below -4.00. A correlation between the segment number and the value of the power law exponent is not apparent.

The radial intensities obtained from single scattering patterns do not show a smooth decay, but a structured signal with weak scattering maxima and minima. Fig. 5.8a shows a scattering pattern recorded on the edge of the cellular extension (compare 
Fig. 5.2 d) along with dashed white lines indicating the segmentation for azimuthal integration. In Fig. 5.8c the corresponding radial intensities after segment-wise background subtraction are shown. For background subtraction the radial intensities of the averaged empty scattering pattern (cf. Fig. 5.6p) were calculated with
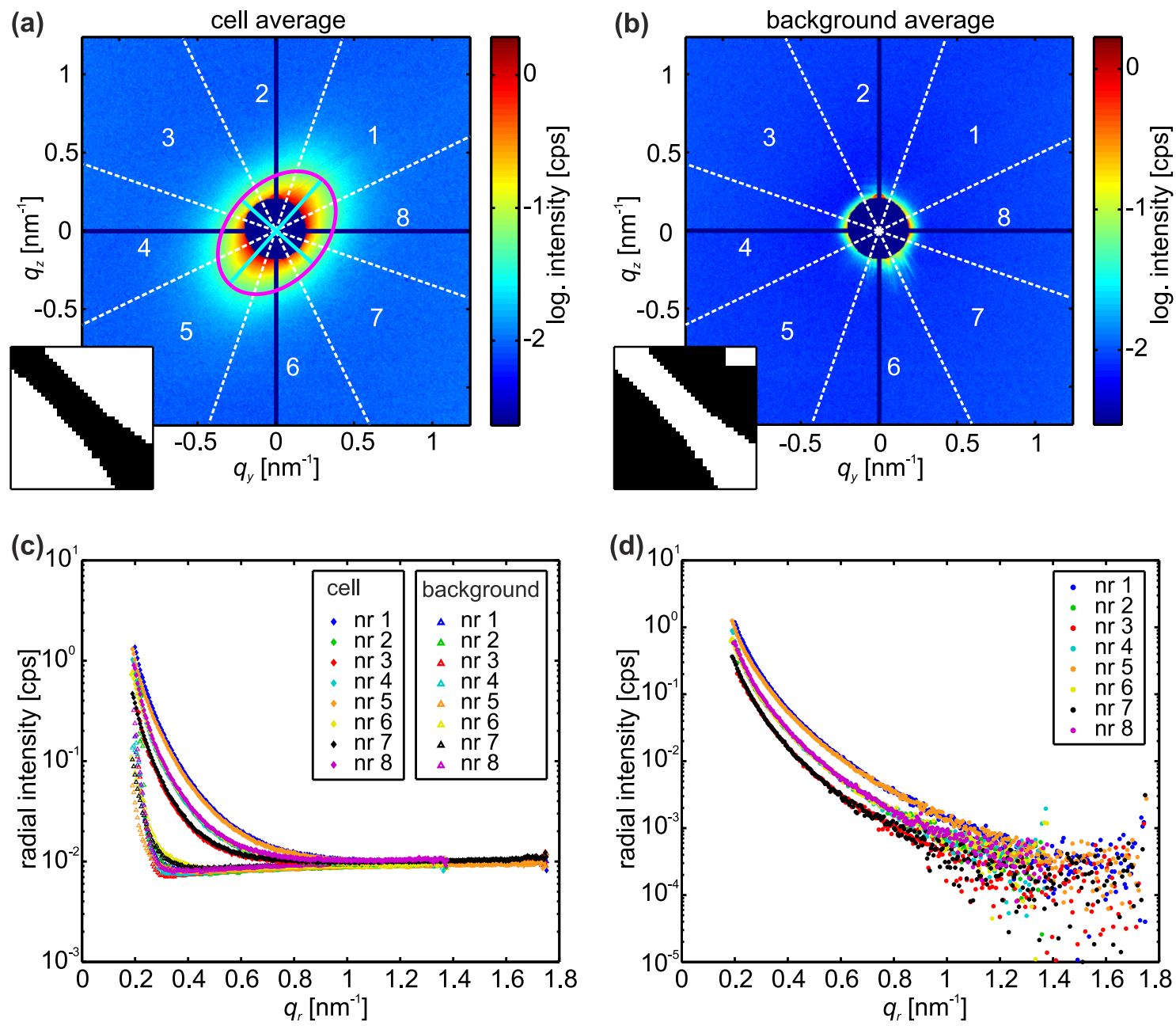

Fig. 5.6.: Azimuthal integration of averaged scattering patterns in eight angular segments for a cell containing a keratin network. (a) Averaged scattering pattern from the keratinrich extension of the cell shown in Fig. 5.2 . The angular segments one to eight, indicated by dashed white lines, were chosen symmetrically around the principle axes (cyan) of the ellipse (magenta) describing the orientation of the scattering signal. (b) Averaged scattering pattern from the empty region. The positions of the angular segments were chosen identically to (a). The insets in (a) and (b) show from which regions of the scan (black pixels) the scattering patterns were used to obtain the average scattering patterns. The side length of the insets is $9.25 \times 9.25 \mu^{2}$. (c) Radial intensity profiles from cell and background regions and (d) segment-wise background corrected radial intensity profiles integrated in eight angular segments. (Sample BW17, position 1; ID13, June 2011) [Figure from [39].] 
(a)

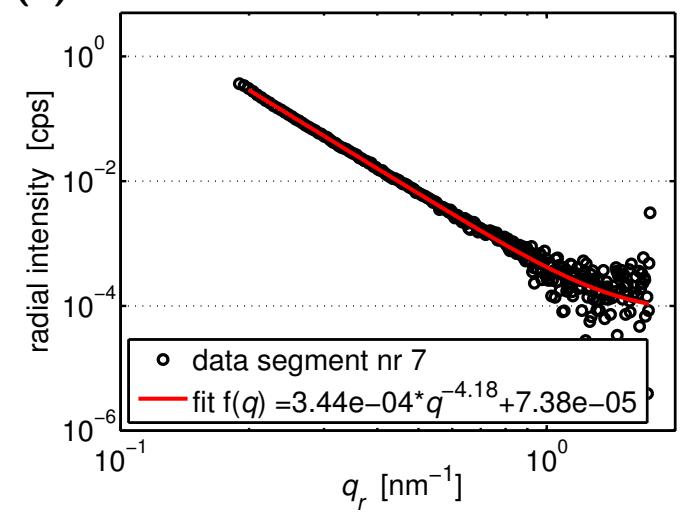

(b)

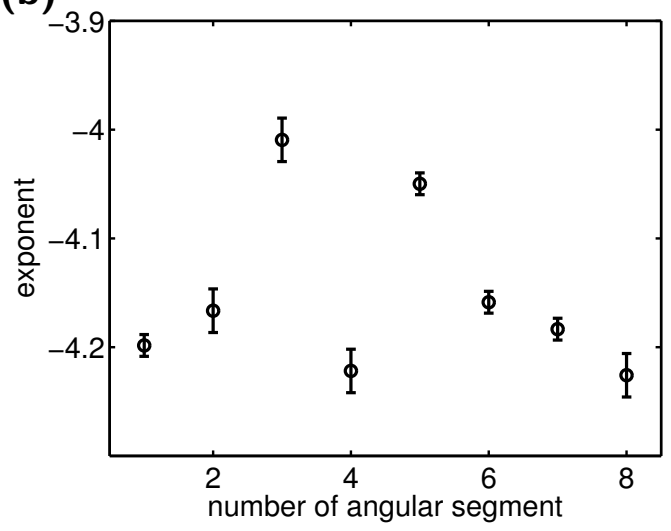

Fig. 5.7.: Fitting of the radial intensity with a power law for a cell with a keratin network. (a) Data points and fit with a power law function exemplarily for the 7th segment. (b) Power law exponents obtained from the fits to all eight angular segments. The error bars indicate the errors obtained from the fits. (Sample BW17, position 1B; ID13, June 2011)

the segmentation of the single scattering pattern and subtracted for each segment separately. Particularly, the radial intensities in the first and fifth segment, which are perpendicular to the local structure direction, show scattering maxima as indicated by arrows in Fig. 5.8. However, it should be pointed out here that not all scattering patterns exhibit peaks or shoulders in the intensity decay and, if peaks are present, the positions are highly heterogeneous. This aspect is also discussed further below in context with Fig. $5.8 \mathrm{~b}$ and d. Scattering patterns recorded on the cell edge are more likely to exhibit peaks or shoulders in the scattering signal, indicating an effect of the edge itself, but also scattering patterns from the inner region of the cellular extension exhibit peaks or shoulders (compare also the composite image in Fig. $5.7 \mathrm{~b})$.

To estimate a corresponding structural sizes in real space from the scattering maxima in Fig. 5.8d, the average radial intensity of the first and fifth angular segment were fitted with two different model functions. Here it was assumed that the scattering corresponds to the form factor of a cylindrical object and not to the (internal) structure factor resulting from, e.g., the filament arrangement in a keratin bundle. Several components of the cell could give rise to a cylinder-like structure, for instance keratin bundles, actin bundles (stress fibers) or mitochondria, but also salt accumulations, i.e. artifact from plunge-freezing as discussed above. For a start, the structure factor is excluded, since the electron density contrast inside a protein bundle, i.e. between individual filaments, is lower than the electron density contrast between the full bundle and the surrounding medium. In the first approach the squared form factor of a cylinder with radius $R$ in direction perpendicular to 

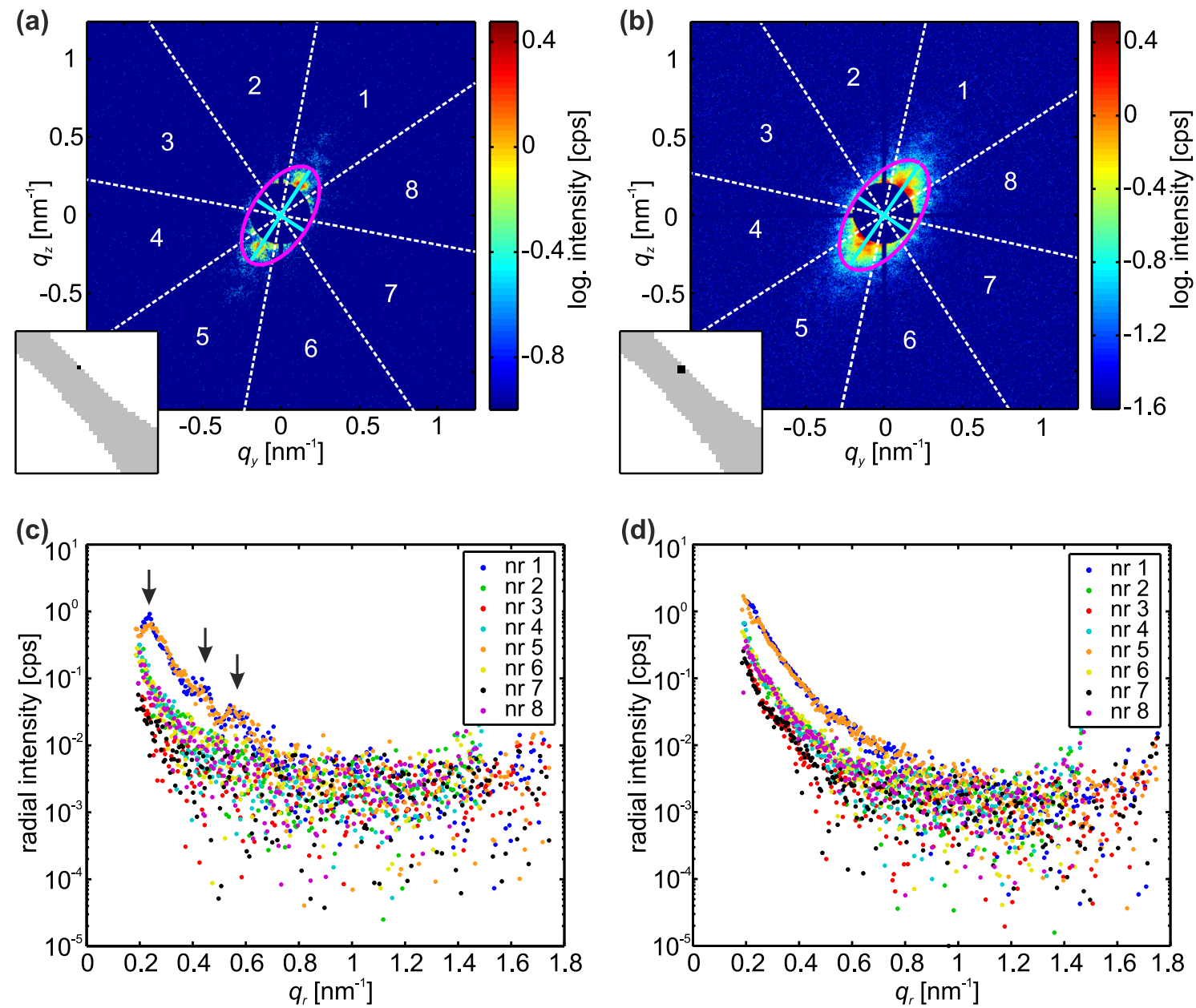

Fig. 5.8.: Azimuthal integration of a single scattering pattern and an average of $2 \times 2$ scattering patterns in eight angular segments for a cell containing a keratin network. (a) Single scattering pattern from the edge of the keratin-rich extension and (b) average of $2 \times 2$ scattering patterns including the scattering pattern shown in (a). The angular segments one to eight, indicated by dashed white lines, were chosen symmetrically around the principle axes (cyan) of the ellipses (magenta) describing the orientation of the scattering signal in each image. The insets in (a) and (b) show the positions (black pixels) of the displayed scattering patterns in the scan. The side length of the insets is $9.25 \times 9.25 \mu \mathrm{m}^{2}$. (c) Radial intensity profiles from a single scattering pattern and (d) from an average of $2 \times 2$ scattering patterns after background subtraction. (Sample BW17, position 1B; ID13, June 2011) [Figure from [39].]

the cylinder axis, i.e.

$$
h(q)=A \cdot\left(\frac{\mathbf{J}_{1}(q R)}{q R} \cdot R^{2}\right)^{2}+C,
$$

was used, with $\mathbf{J}_{1}$ being the first order Bessel function of the first kind, $A$ a scaling factor and $C$ an additive background. Fig. $5.9 \mathrm{a}$ shows the fit along with the data. 
(a)

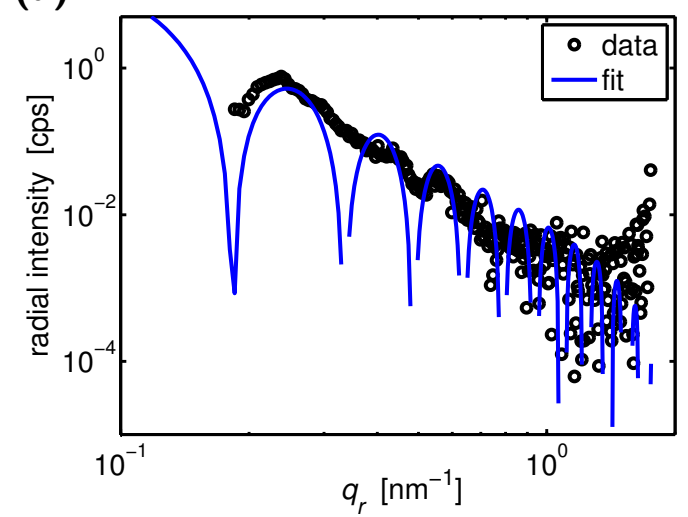

(b)

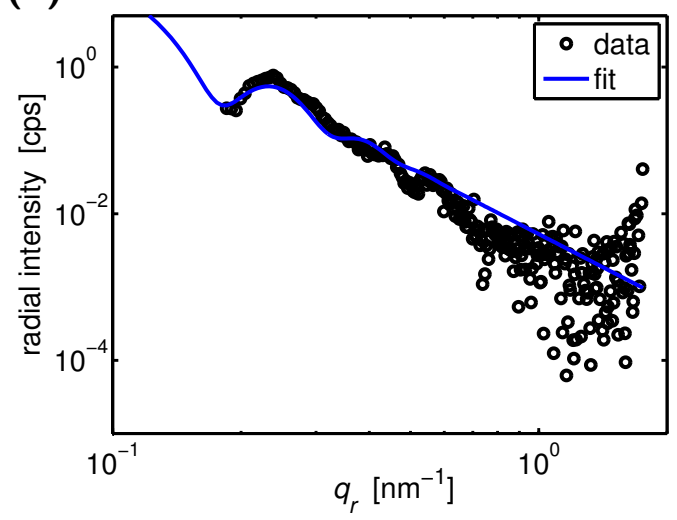

Fig. 5.9.: Fitting of the average the first and fifth radial intensity profile of a single scattering pattern. (a) Data and fit using the form factor of a cylinder in direction perpendicular to the cylinder axis as model function. (b) Data and fit using the form factor of a cylinder with a radial polydispersity as model function. (Sample BW17, position 1B; ID13, June 2011)

This approximation of the scattered intensity yielded a value of $R=21 \mathrm{~nm}$ which is in the biologically relevant order of magnitude. Since the intensity maxima of the fit function and the data coincide well, the choice of the form factor of a cylinder seems to be the correct basic principle to describe the local structure in this scattering pattern. Fig. 5.9b shows a fit with a model function for a cylinder with a radial polydispersity. This second fit yielded a value of $R=21 \mathrm{~nm}$ as well and a radial polydispersity parameter about $2.6 \mathrm{~nm}$, which is also in the biologically relevant range. Deviations of the radial intensity from the cylindrical approximation can be explained by the fact that the probed sample structure is not described by a perfect cylinder, but by a much more complex arrangement of proteins on different length scales. For a better fit, the detailed substructure needed to be taken into account, i.e. the filament arrangement and the filament form factor if the cylinder really corresponds to a keratin bundle. Furthermore, as mentioned above and discussed in more detail in the next paragraph, the structure in the cell is highly heterogeneous. Therefore several model functions would be needed to describe different positions in the cell and also the superposition of scattering from different cellular structures. Additionally, the beam size that is here in the same order of magnitude as the structure sizes in the sample should be considered in the fit as well, which would lead to a smoothing of the peaks in the radial intensity profiles (compare section 3.1). However, to obtain an estimate for the structure size in the cellular sample based on the recorded scattering signal, the approach presented here is an appropriate method.

In contrast to the above observation, the same analysis applied to an average of 
$2 \times 2$ adjacent scattering patterns, including the scattering pattern shown in Fig. 5.8 a, reveals a smoother decay of the radial intensity profiles without pronounced maxima or minima in the scattering signal (see Fig. $5.8 \mathrm{~b}, \mathrm{~d}$ ). This finding indicates small changes in the peak positions, which result in a smooth intensity decay after averaging. Therefore, the detailed intensity distribution in the scattering patterns in reciprocal space and correspondingly also the local sample structure in real space, sensitively depend on the position on the sample. Taking into account the beam size and step size during the scan, the average of $2 \times 2$ scattering patterns covers an area of about $400 \times 400 \mathrm{~nm}^{2}$ on the sample and thus the maximum beam size for probing the local structure in this type of samples needs to be smaller than (at least) $400 \times 400 \mathrm{~nm}^{2}$.

\subsubsection{Cells without a Keratin Network}

\subsubsection{X-Ray Dark-Field Images and Structure Orientation}

Measurements with the same combinations of step sizes and exposure times as employed for the region of interest measurement on cells with keratin, were performed on a cell without a keratin network. Fig. 5.10c shows an X-ray dark-field image on a group of SK8/18-2 cells reconstructed from a coarse scan with a step size of $2 \mu \mathrm{m}$ and $0.1 \mathrm{~s}$ exposure time. Only the cell on the right hand side of the image contains a keratin network, as validated by the visible light fluorescence microscopy image of the keratin network in Fig. 5.10a. The fluorescence microscopy image was taken on the hydrated cell, after fixation with formaldehyde, but before plunge-freezing and
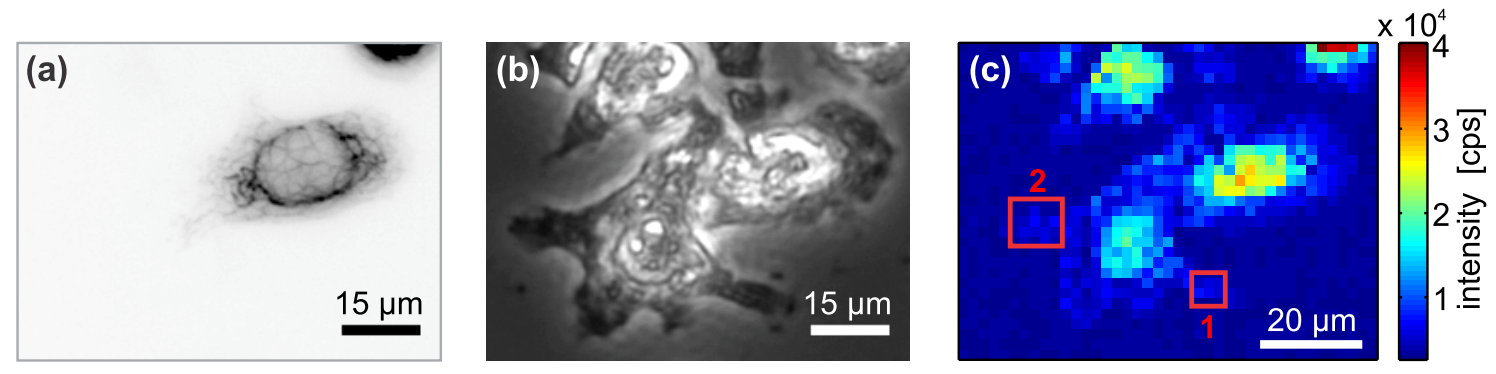

Fig. 5.10.: Microscopy images and coarse X-ray dark-field image of a group of freeze-dried SK8/18-2 cells (a) Fluorescence microscopy image of the keratin network in the hydrated cells and (b) phase contrast microscopy image of the same cells after freeze-drying. The cell in the central-left part of the image does not contain a keratin network. (c) X-ray dark-field image on the same sample region reconstructed from a mesh scan with a step size of $2 \mu \mathrm{m}$ and $0.1 \mathrm{~s}$ exposure time. On the marked regions $(1,2)$, finer scans with longer exposure times were performed. (Sample BW21, position 6A; ID13, Nov. 2011) 
(a)

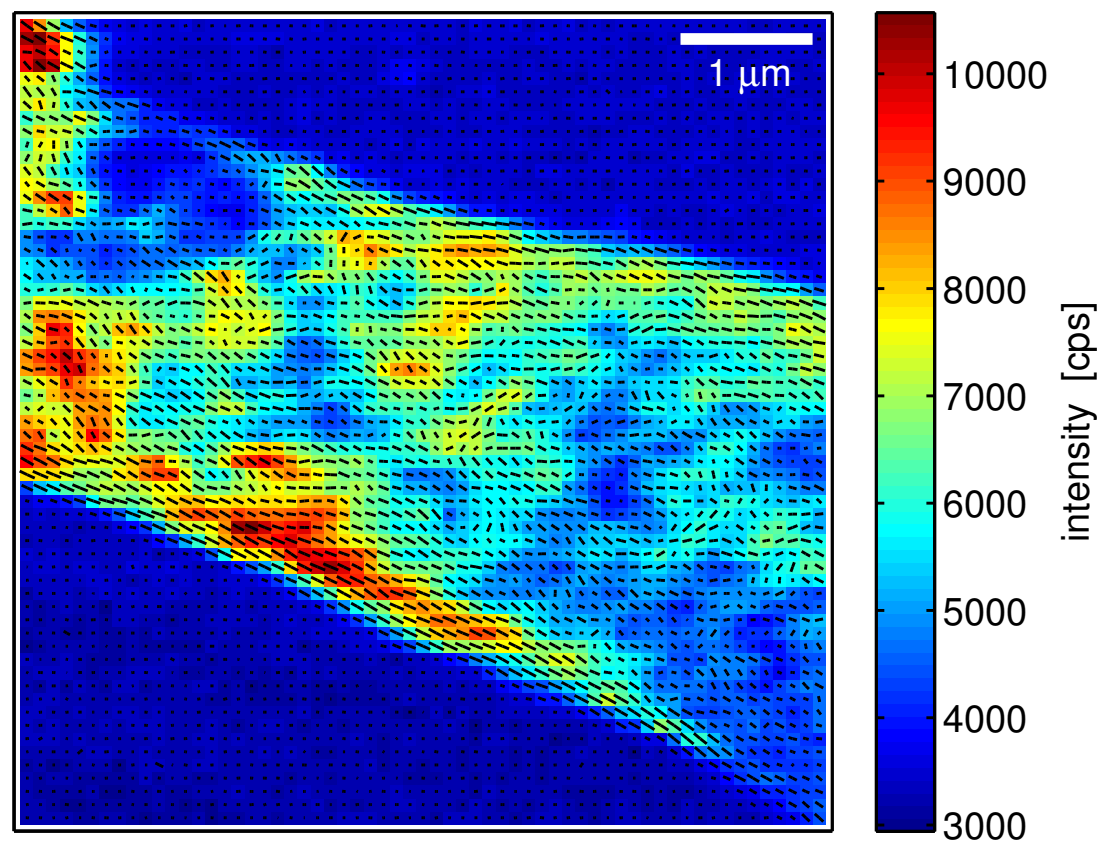

(b)
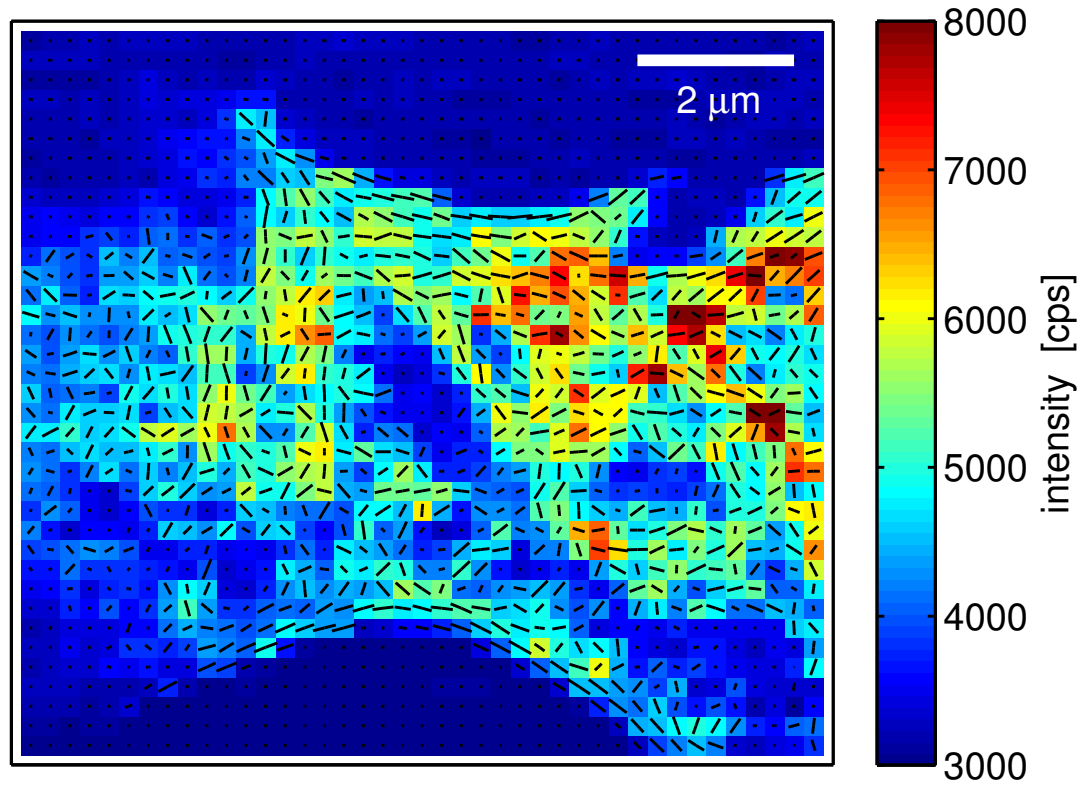

Fig. 5.11.: Structure orientation and X-ray dark-field image of the cellular extensions of a keratin-free freeze-dried SK8/18-2 cell. (a) Structure orientation map as overlay with X-ray dark-field image of the cellular extension, which is marked as region 1 in Fig. 5.10. During this scan a step size of $100 \mathrm{~nm}$ and an exposure time of $1 \mathrm{~s}$ were employed. (b) Structure orientation map as overlay with X-ray dark-field image of the cellular extension, which is marked as region 2 in Fig. 5.10k. During this scan a step size of $250 \mathrm{~nm}$ and an exposure time of $10 \mathrm{~s}$ were employed. (Sample BW21, position 6B, C; ID13, Nov. 2011) 
freeze-drying. A visible light phase contrast microscopy image of the same sample region after freeze-drying is shown in Fig. 5.10b.

Region of interest scans with smaller step sizes of $100 \mathrm{~nm}$ and $250 \mathrm{~nm}$ and longer exposure times of $1 \mathrm{~s}$ and $10 \mathrm{~s}$, respectively, were performed on regions of the cellular extensions that are marked by red squares in Fig. 5.10k. The corresponding X-ray dark-field images along with maps of the structure orientation in the sample are shown in Fig. 5.11. The X-ray dark-field images of the keratin-free cell show substructures in the cellular extension, which are indicated by regions of higher or lower scattering intensity. Compared to the analysis of the keratin-containing cell, which is displayed in Fig. 5.5d, the substructures in the keratin-free cell are larger. Furthermore, the intensity distribution in the X-ray dark-field image of the keratin-free cell does not show network-like pattern, as it was observed for the keratin-containing cell. Considering the possibility of artifacts due to plunge-freezing and freeze-drying, the larger structures in the cellular extension could correspond to local accumulations of cellular material caused by the formation of crystalline ice during plunge-freezing instead of vitreous ice.

\subsubsection{Radial Intensities of Averaged Scattering Patterns}

For the region of interest measurement with an exposure time of $10 \mathrm{~s}$, which is shown in Fig. 5.11b, all scattering patterns on the cellular extension and on the background region, respectively, were averaged yielding the scattering patterns presented in Fig. 5.12 and $b$. The insets indicate from which regions of the scan (black pixels) the scattering patterns were used for averaging. The averaged scattering pattern from the background region shows scattering from the aperture above and below the beamstop in the center of the image. The averaged scattering from the cell is much stronger than the background signal.

The averaged scattering patterns were integrated in azimuthal directions in one angular segment, resulting in the radial intensity profiles shown in Fig. 5.12k. The radial intensity profile from the cell is well above the background signal. Here, the azimuthal integration was performed in one angular segment, because on the one hand the scattering signal is nearly isotropic and on the other hand the eight different angular segments employed for the keratin-containing cell yielded similar power law exponents for the intensity decay (compare Fig. 5.7b). The background corrected radial intensity profile can be well described by a power law over the whole $q_{r}$-range. The resulting fit function is displayed in Fig. $5.12 \mathrm{~d}$ along with the data. Here, the obtained power law exponent of -4.32 is slightly smaller than the power law exponents obtained for the keratin-containing cell, but it is still very close to this value. A further classification of the differences or similarities between the power 
law exponents for all measurements on freeze-dried samples is given in the discussion at the end of this chapter.
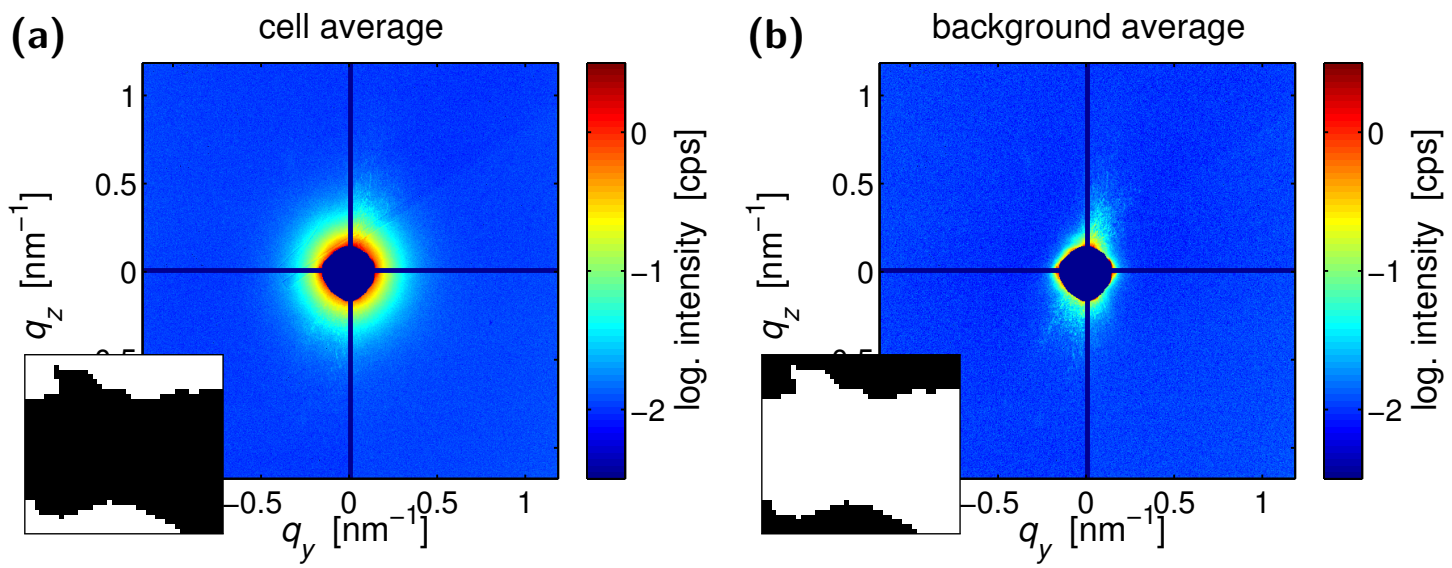

(c)

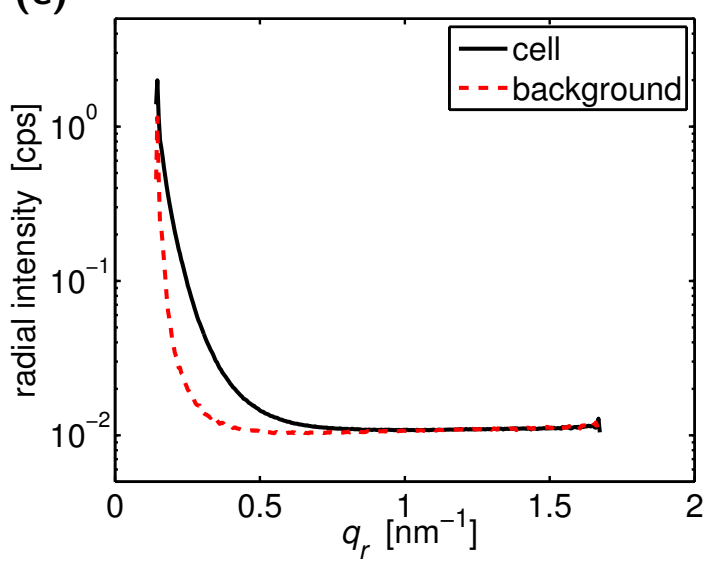

(d)

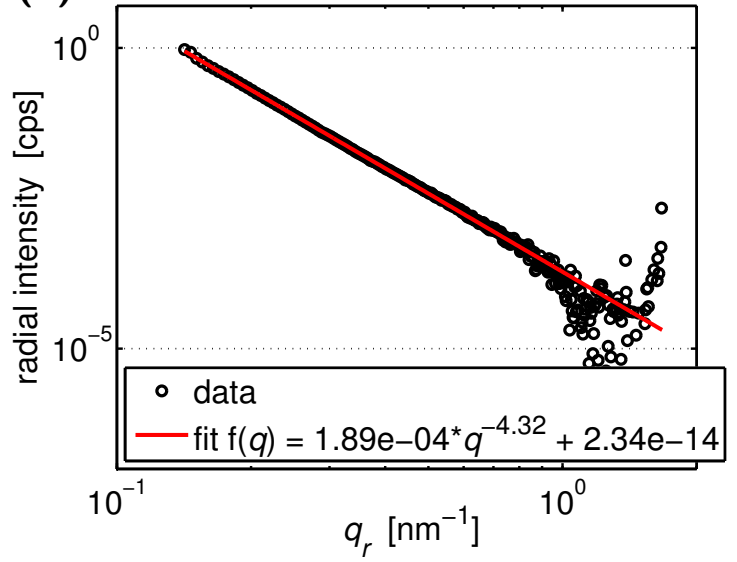

Fig. 5.12.: Azimuthal integration of averaged scattering patterns for a keratin-free cell. (a) Averaged scattering pattern from the cellular extension marked as region 2 in Fig. 5.10. (b) Averaged scattering pattern from the empty region. The insets in (a) and (b) show from which regions of the scan (black pixels) the scattering patterns were used to obtain the average scattering patterns. The size of the inset is $10 \times 9 \mu^{2}$. (c) Radial intensity profiles from the cell and the background region. (d) Fitting of the background corrected radial intensity profiles with a power law. (Sample BW21, position 6C; ID13, Nov. 2011)

\subsubsection{Cells treated with the IF Lysis Buffer}

\subsubsection{X-Ray Dark-Field Images and Structure Orientation}

Similar measurements were performed on a sample that was treated with IF lysis buffer, which leads to a disassembly of all cytoskeletal components except for the keratin network and a removal of all soluble components from the cytosol (compare 
section 4.2.5). Fig. 5.13 a shows the visible light fluorescence microscopy image of the keratin network in a group of SK8/18-2 cells and the corresponding phase contrast microscopy image taken on the same sample region after freeze-drying is shown in panel b. The X-ray dark-field image that was reconstructed from a coarse mesh scan with a step size of $2 \mu \mathrm{m}$ and an exposure time of $0.1 \mathrm{~s}$ is presented in Fig. 5.13. Compared to the coarse X-ray dark-field images of the non-treated cells discussed above, the image of the cell treated with the IF lysis buffer is much noisier and the
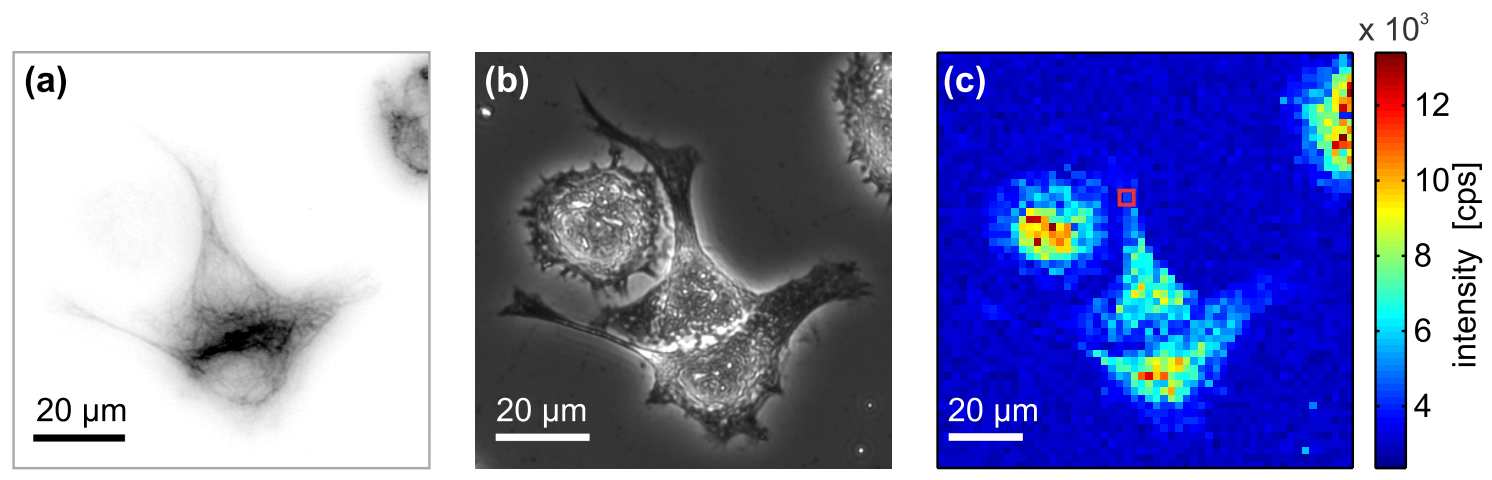

(d)

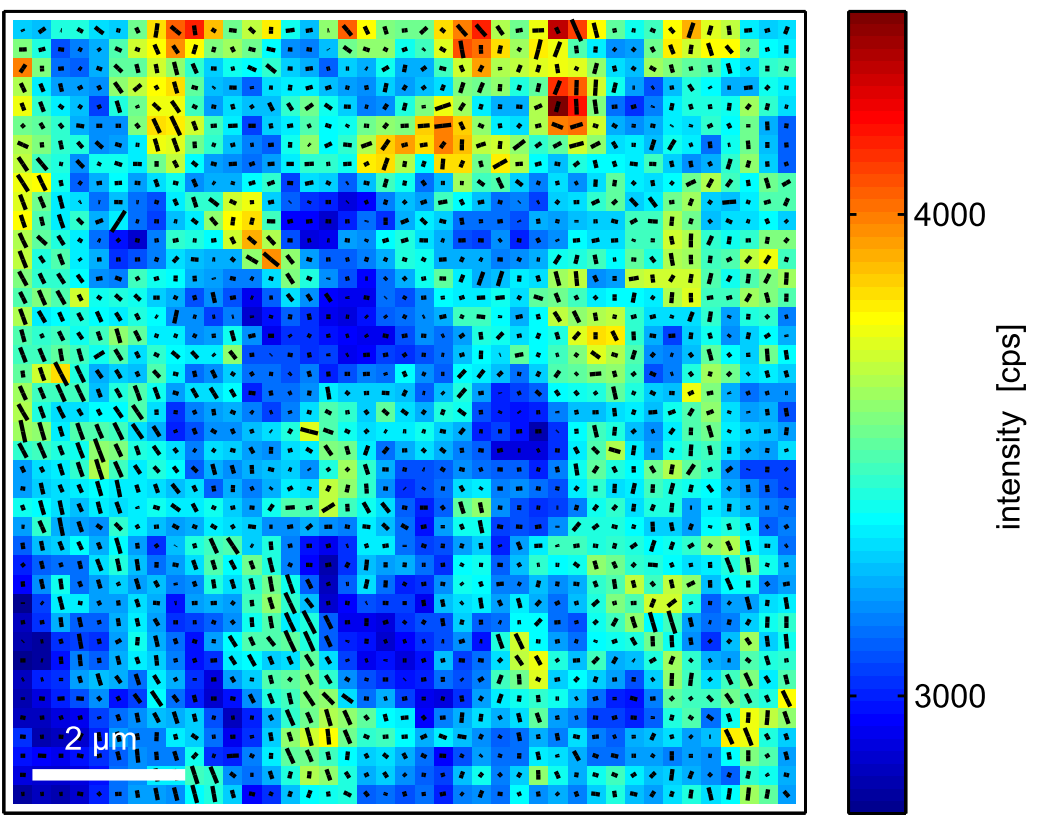

Fig. 5.13.: X-ray dark-field image and structure orientation in freeze-dried cells that was treated with IF lysis buffer. (a) Fluorescence microscopy image of the keratin network taken before plunge-freezing and freeze-drying and (b) phase contrast microscopy image of the same sample region taken after freeze-drying. (c) Coarse X-ray dark-field image reconstructed from a scan with a step size of $2 \mu \mathrm{m}$ and $0.1 \mathrm{~s}$ exposure time. (d) Structure orientation map as overlay with the X-ray dark-field image from a scan with $100 \mathrm{~nm}$ step size and $1 \mathrm{~s}$ exposure time on the cellular extension in the region that is marked by a red square in panel (c). (Sample BW54, position 1; ID13, June 2011) 
cellular extensions, e.g., at the upper part of the cell in the center of the image, can hardly be identified.

A finer mesh scan with a step size of $100 \mathrm{~nm}$ and an exposure time of $1 \mathrm{~s}$ was performed on the cellular extension at the top of the cell. The X-ray dark-field image along with a map of the structure orientation in the cellular extension is shown in Fig. 5.13d. Also for this measurement, the intensity is lower and noisier compared to similar measurements on the non-treated cells. However, the local structure orientation in the sample could be determined applying the method presented above, but it is more sensitive to artifacts in the scattering signal (e.g. traces from cosmic particles on the detector) due to the lower scattering signal. The local structure orientation as indicated by the black lines in Fig. 5.13d agrees very well with the orientation of larger structures that are visible in the X-ray dark-field image. The apparent structure sizes are in a range of $100-400 \mathrm{~nm}$, which is similar to the image of the untreated cell. Due to the low signal-to-noise ratio, a network-like morphology cannot be clearly identified.

\subsubsection{Radial Intensities of Averaged Scattering Patterns}

All scattering patterns from the measurement on the cellular extension were averaged yielding the scattering pattern shown in Fig. 5.14a. For the averaged scattering pattern from the background region, shown in Fig. 5.14 b, 50 exposures with the same exposure time as employed for the measurements on the cellular extension were recorded on a separate empty position and averaged. Both images were integrated in azimuthal direction, resulting in the two radial intensity profiles shown in Fig. 5.14c. Here, again, only one angular segment was used for the azimuthal integration since no difference in the power law exponents was previously observed for different segments. The radial intensity profile from the background position shows a high noise level in the signal, indicating that more exposures and maybe at different empty positions should have been recorded. Furthermore, the radial intensity profile from the background position exceeds the signal from the cells at high $q_{r}$-values, which is probably related to changes in the ring current (no top-up mode) or it is an effect of the low photon statistic in the averaged background scattering pattern.

The background corrected radial intensity profile from the cell was fitted with a power law function. The fit function is depicted in Fig. $5.14 \mathrm{~d}$ along with the background corrected radial intensity profile. The radial intensity profile is more noisy than the profiles for the untreated cell, which can be attributed to the shorter exposure time, the treatment with IF lysis buffer and a noisier background signal. From the fit, a power law exponent of -4.17 is obtained, which is similar to the power 

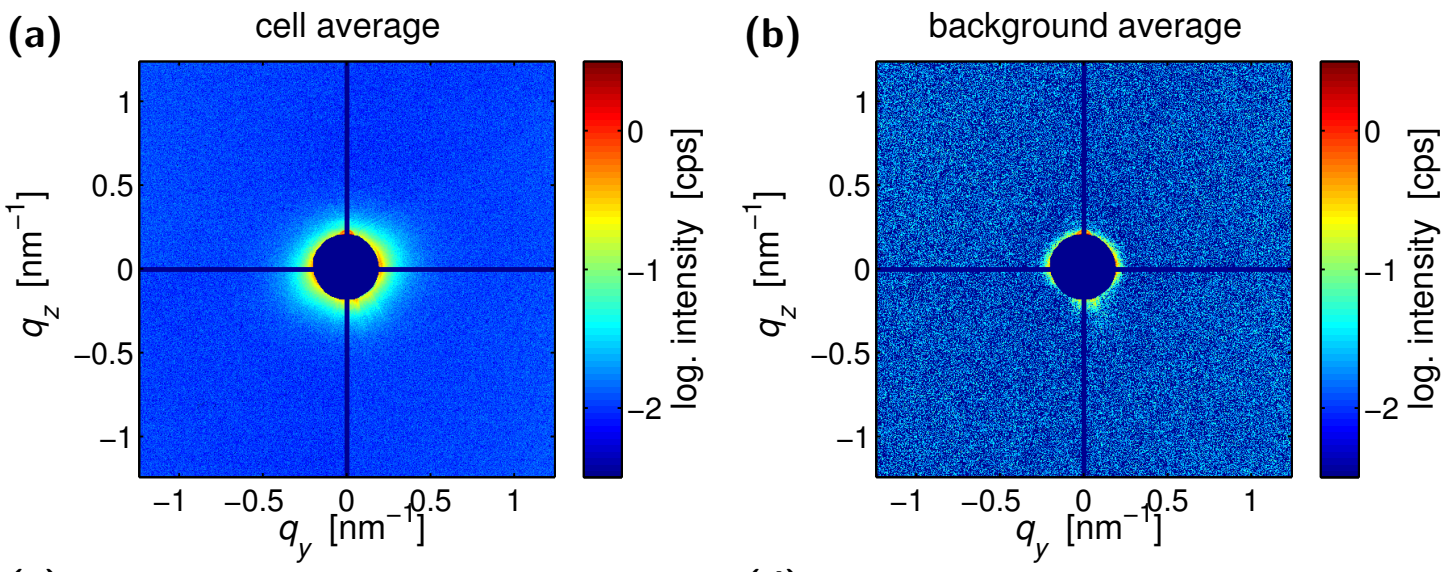

(c)
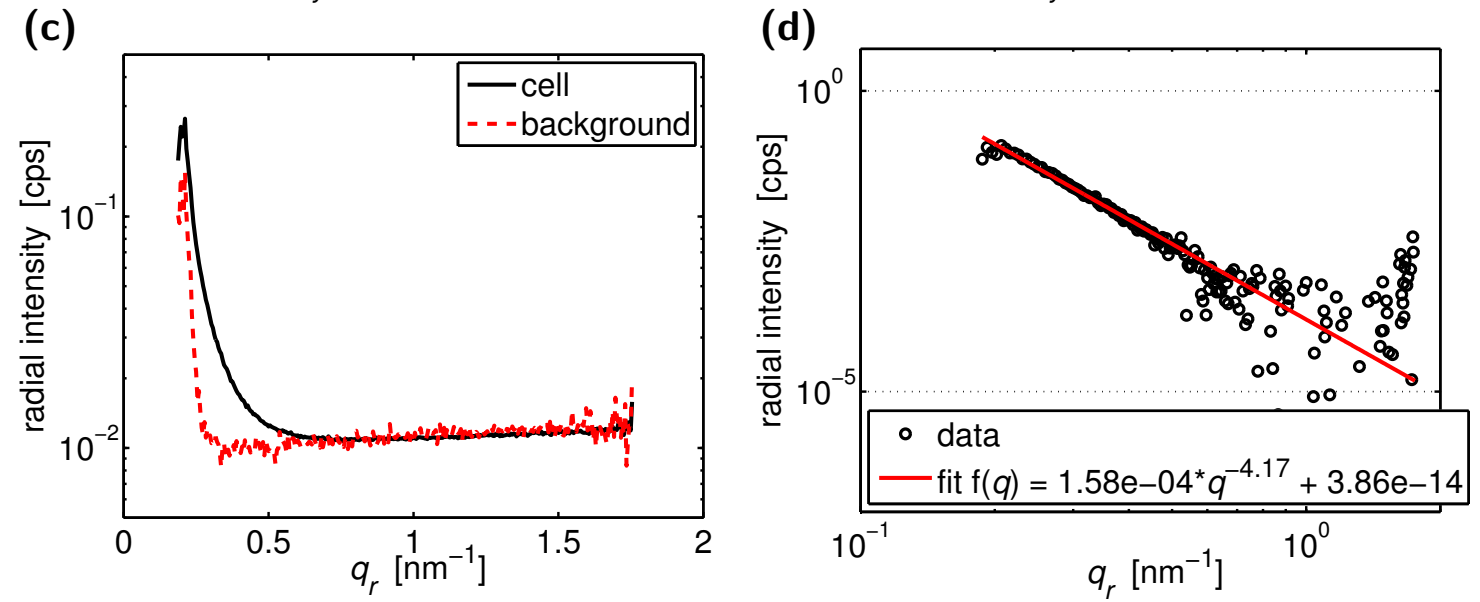

Fig. 5.14.: Azimuthal integration of averaged scattering patterns for a cell that was treated with the IF lysis buffer. (a) Averaged scattering pattern from the region of interest scan shown in Fig. 5.13c. (b) Averaged scattering pattern from a separate scan on an empty position. (c) Radial intensity profiles from the cell and the background region. (d) Fit of the background corrected radial intensity profiles with a power law. (Sample BW54, position 1B; ID13, June 2011)

law exponent obtained for the non-treated cells with keratin. Therefore, a difference between the treated and the untreated cells cannot be determined from the decay of the radial intensity of averaged scattering patterns. 


\subsection{Results from Measurements at the P10 Beamline}

At the P10 beamline experiments were performed only on cells with a keratin network and keratin-free SK8/18-2 cells, which were both not treated with the IF lysis buffer. In the following part, measurements and results for one example of a cell with a keratin network are presented. Additional X-ray dark-field images as well as average radial intensity profiles and the corresponding fits with power laws, are documented in the supplementary material in section B.1.

An overview of the analyzed measurements on freeze-dried SK8/18-2 cells taken at the P10 beamline along with the employed scan parameters and the estimated average radiation doses per step is shown in Tab.5.2.

\begin{tabular}{lllrlll}
\hline sample & set-up & $\Delta_{y, z}\left[\mu \mathrm{m}^{2}\right]$ & $N_{y} \times N_{z}$ & $T[\mathrm{~s}]$ & $T_{\mathrm{att}}$ & $D[\mathrm{~Gy}]$ \\
\hline BW129, pos. 3 & Oct. 2012 & $2 \times 2$ & $81 \times 121$ & 0.05 & 0.263 & $6.4 \times 10^{5}$ \\
BW130, pos. 1 & Oct. 2012 & $0.5 \times 0.5$ & $321 \times 281$ & 0.04 & 1 & $3.1 \times 10^{7}$ \\
BW130, pos. 2 & Oct. 2012 & $0.5 \times 0.5$ & $121 \times 201$ & 0.05 & 1 & $3.9 \times 10^{7}$ \\
BW201, pos. 2 & March 2013 & $1 \times 1$ & $101 \times 141$ & 0.05 & 0.263 & $1.9 \times 10^{6}$ \\
BW201, pos. 3 & March 2013 & $0.5 \times 1$ & $81 \times 101$ & 0.05 & 1 & $1.4 \times 10^{7}$ \\
\hline
\end{tabular}

Tab. 5.2.: Summary of the scan parameters and the estimated average radiation doses per step for the measurements on freeze-dried cells at the P10 beamline. Here, $\Delta_{y, z}$ are the lateral step sizes, $N_{y}$ and $N_{z}$ are the number of scan points in $y$ - and $z$-direction, $T$ is the exposure time, $T_{\text {att }}$ the attenuator transmission and $D$ the estimated average radiation dose per step.

\subsubsection{X-Ray Dark-Field Images and Structure Orientation}

An example for a measurement performed at the P10 beamline is shown in Fig. 5.15 . Fig. 5.15 a shows the visible light fluorescence microscopy image of the keratin network in a SK8/18-2 cell, which exhibits long cellular extensions that contain keratin. A phase contrast microscopy image of the same sample region after freeze-drying is depicted in Fig. 5.15b, showing that the shape of the cell in the center of the imaged is very well preserved during plunge-freezing and freeze-drying. However, in the group of cells in the lower left corner of the image, several cracks, which occurred during plunge-freezing and freeze-drying, are visible. The corresponding X-ray dark-field image of the same sample region is shown in Fig. 5.15c. In the X-ray dark-field image, the cells are clearly visible and the nuclei can be distinguished from the cell body. Also thin cellular extensions can be identified. To visualize further 

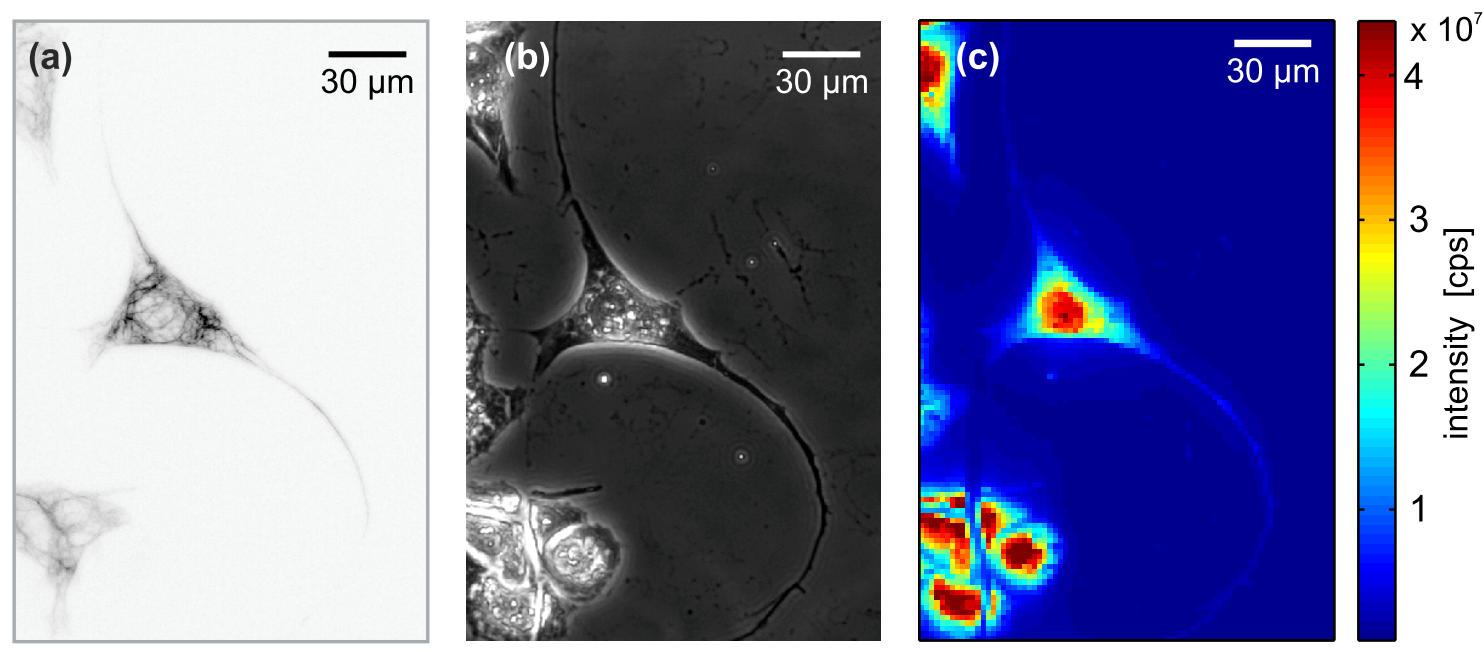

Fig. 5.15.: Microscopy images and X-ray dark-field image of a freeze-dried SK8/18-2 cell with a keratin network. (a) Fluorescence microscopy image of the keratin network taken before plunge-freezing and freeze-drying and (b) phase contrast image after freeze-drying. (c) X-ray dark-field image of a scan with a step size of $2 \mu \mathrm{m}$ and an exposure time of $0.05 \mathrm{~s}$ on the same sample region. (Sample BW129, position 3; P10, Oct. 2012)

cellular substructures, the resolution employed here, i.e. the step size of $2 \mu \mathrm{m}$, is not high enough. For the measurements using finer step sizes (compare Tab. 5.2), substructure were visible in the nucleus as well as in the cell body (see appendix B.1 for the X-ray dark-field images). Due to the high photon flux and the strong radiation damage to the samples at the $\mathrm{P} 10$ beamline, no reliable region of interest measurement could be performed on the cells. In particular, the thin cellular extensions were destroyed during the first coarse measurement.

To determine the direction and the degree of the structure orientation in the sample from the individual scattering patterns, the same approach as presented in section 5.2.1.1 for the data recorded at the ID13 beamline was applied. Two-dimensional maps of the direction and degree of the local structure orientation in the cell are presented in Fig. 5.16a and b, respectively. In both images the cells can be identified. In particular, the degree of structure orientation as indicated by the eccentricity of the fitted ellipses is much higher on the cell than on the empty regions. The thin cellular extensions and the nucleus can be clearly identified in this image, even better than in the X-ray dark-field image.

Fig. 5.17 shows an overlay of the X-ray dark-field image with the vector field combining the degree and direction of structure orientation in the sample. On the cellular extension the local structure orientation fits well to the direction of the cellular extensions. However, on the cell body the obtained local structure orientations have a strong preference for the vertical direction. This can be probably explained by 
(a)

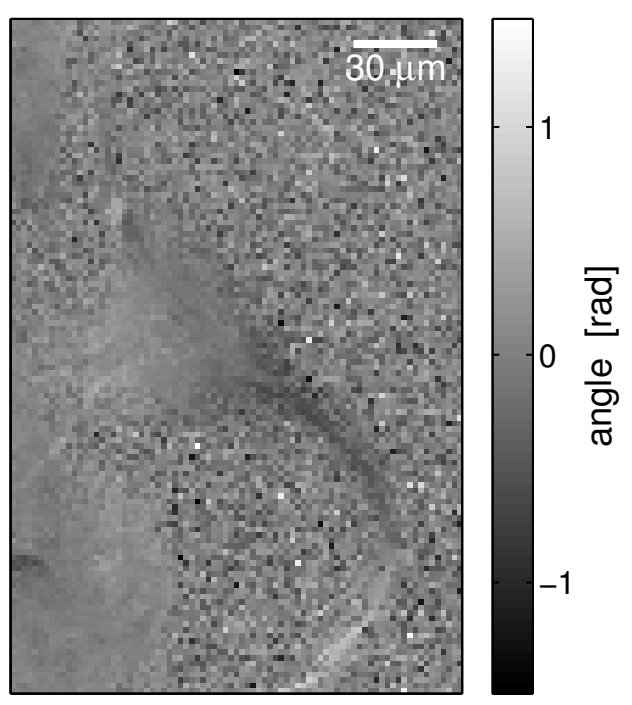

(b)

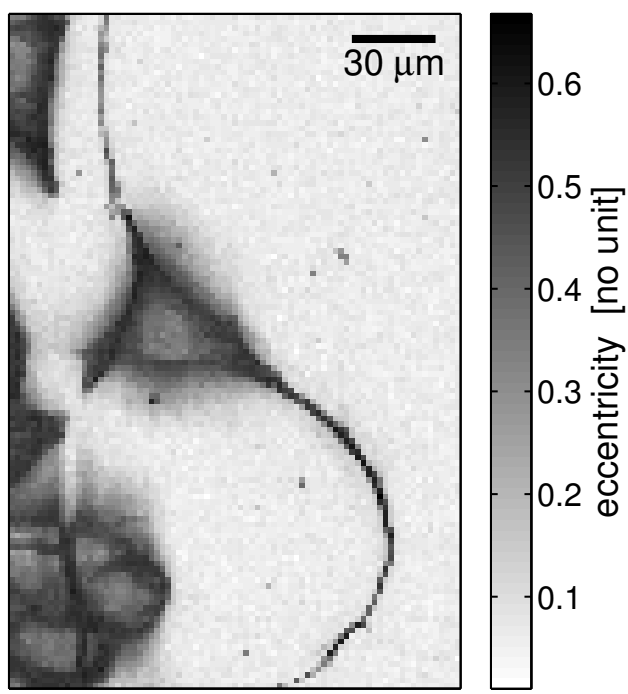

Fig. 5.16.: Maps of (a) the direction and (b) the degree of structure orientation in the sample as determined by approximating the thresholded intensity in each scattering pattern with an ellipse that has the same second moments as the intensity distribution. (Sample BW129, position 3; P10, Oct. 2012)

the asymmetry of the KB beam having a larger size in horizontal than in vertical direction, which will also lead to an anisotropy in each scattering patterns. Only for highly anisotropic scattering from the sample, e.g., on the cellular extension, the anisotropy of the beam does not dominate the total anisotropy of the scattering pattern. An attempt to correct for the preferred orientation in the scattering patterns, led to further artifacts in orientation (not shown). The streaks from the $\mathrm{KB}$ beam and the apertures in the scattering patterns (compare Fig. 5.18d) also led to artifacts during this analysis and needed to be masked. Since quite a large region of the scattering pattern needed to be masked, this probably also influenced the analysis.

\subsubsection{Radial Intensities of Averaged Scattering Patterns}

Averaged scattering patterns were calculated for three different regions of the scan, i.e. the nucleus, the cell body and an empty region as indicated in Fig. 5.18a. The corresponding averaged scattering patterns are presented in Fig. $5.18 \mathrm{~b}-$ d. Both, the nucleus and the cell body show a strong and isotropic scattering signal. However, the scattering from the nucleus is more intense than the scattering from the cell body, as expected from the X-ray dark-field image. In the average scattering patterns from the empty region, the background scattering from the KB optics and the 


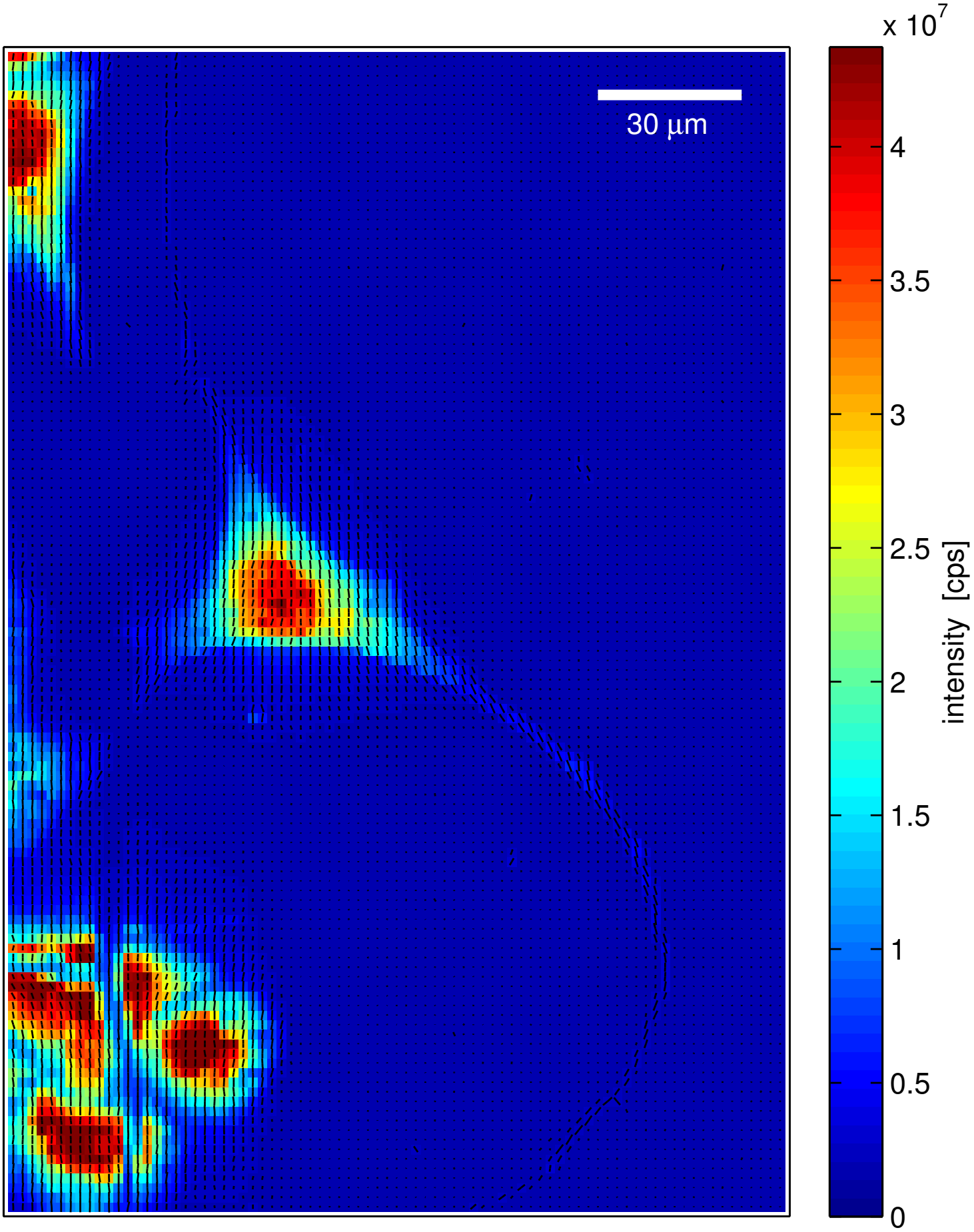

Fig. 5.17.: Overlay of the X-ray dark-field image and the vector field combining the direction and the degree of local structure orientation in the sample. (Sample BW129, position 3; P10, Oct. 2012) 
(a)

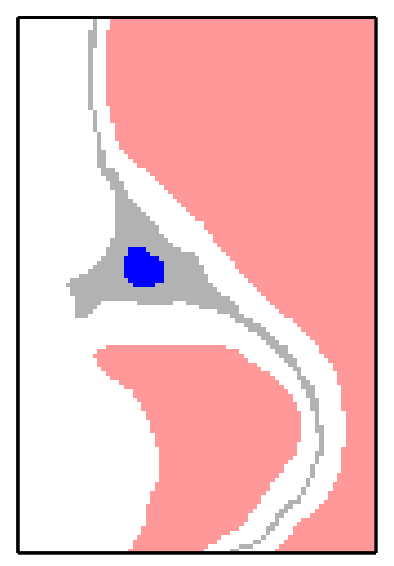

(c)

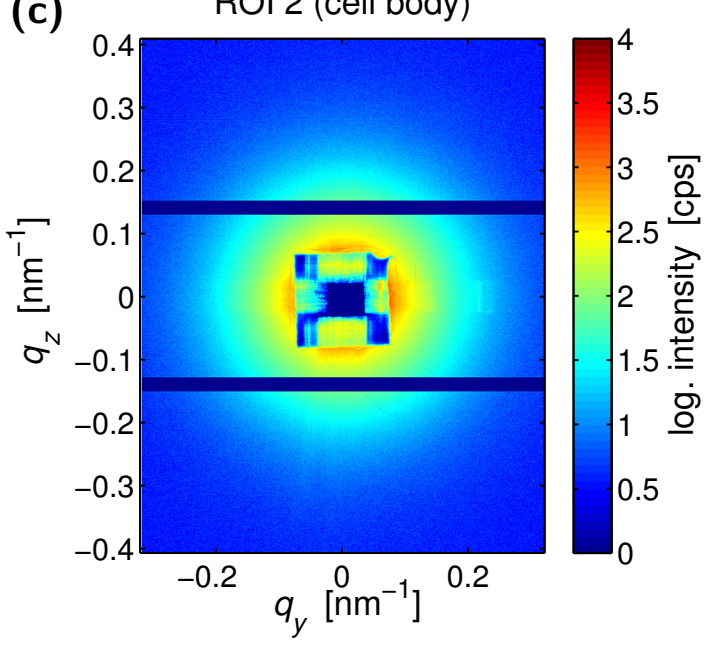

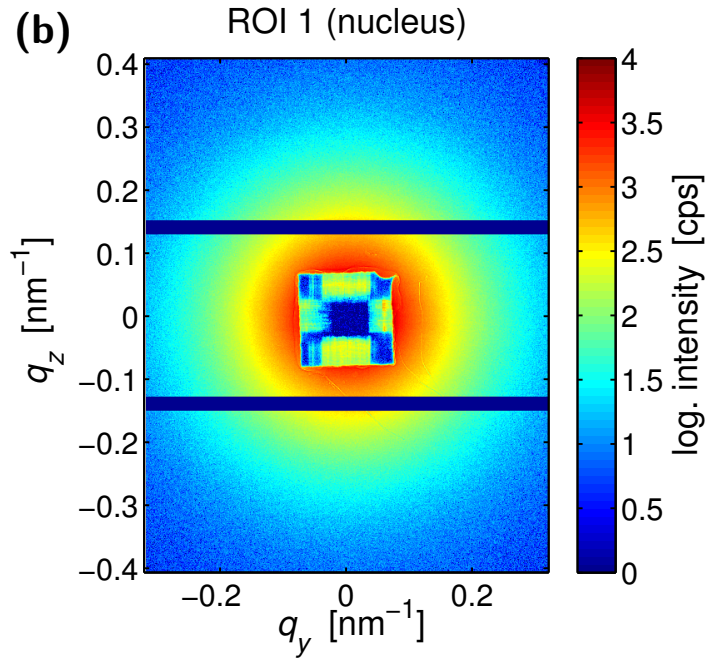

(d)

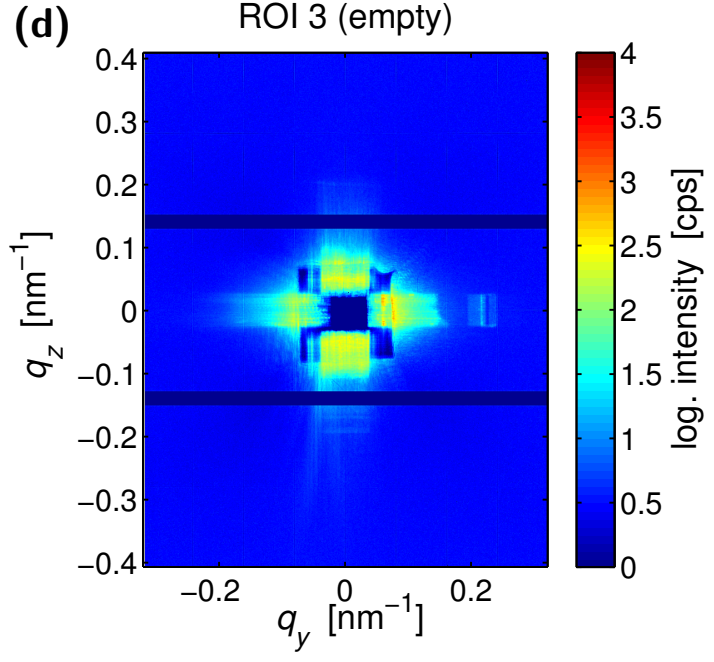

Fig. 5.18.: Averaged scattering patterns from three different scan regions. (a) Selection of three different scan regions, i.e., the nucleus (blue, ROI 1), the cell body (gray, ROI 2) and an empty region (red, ROI 3). Averaged scattering pattern from (b) the nucleus, (c) the cell body and (d) the empty region. (Sample BW129, position 3; P10, Oct. 2012)

soft-edge apertures in form streaks, as well as a weak scattering around the beamstop are visible. Apart from the streaks the background scattering is very low and flat at higher $q$-values.

The averaged scattering patterns were integrated in azimuthal direction in one angular segment, yielding the three radial intensity profiles depicted in Fig. 5.19a. Subtraction of the background scattering from the two profiles obtained on the cell, resulted in the curves shown in Fig. $5.19 \mathrm{~b}$. Both curves show a similar shape, which exhibits a kink in the intensity decay between $0.1 \mathrm{~nm}^{-1}$ and $0.2 \mathrm{~nm}^{-1}$ with a steeper decay at higher $q_{r}$-values.

To quantify the intensity decay, the radial intensity profiles were approximated by power laws. To take into account the kinks in the radial intensity profiles, two power 
(a)

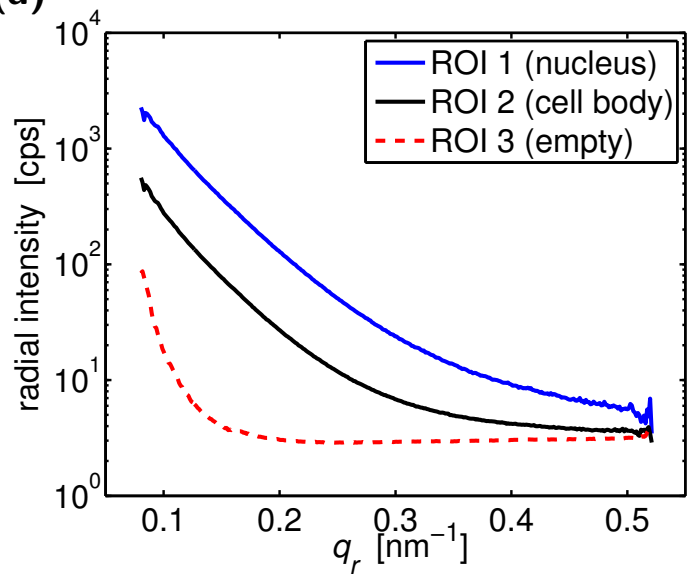

(b)

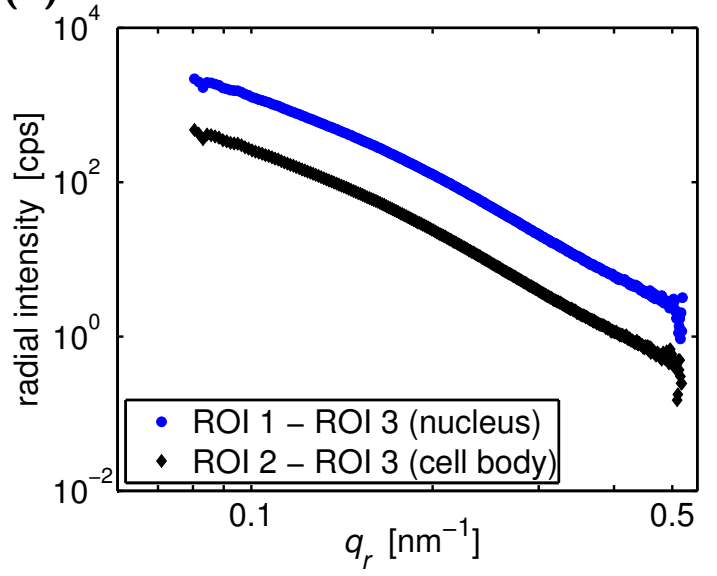

Fig. 5.19.: Radial intensity profiles of averaged scattering patterns. (a) Radial intensity from the nuclear region, the cell body and the empty region. (b) Background corrected radial intensity profiles from the nuclear region and the cell body. (Sample BW129, position 3; P10, Oct. 2012)

law functions were fitted to the regions of high and low $q_{r}$-values, respectively. Fig. 5.20 shows the resulting fit functions along with the background corrected radial intensity profiles. The $q_{r}$-ranges, over which the fits are plotted, correspond to the regions that were used for fitting. In the low and high $q_{r}$-region power exponents of about -3 and -4.5 are obtained, respectively.

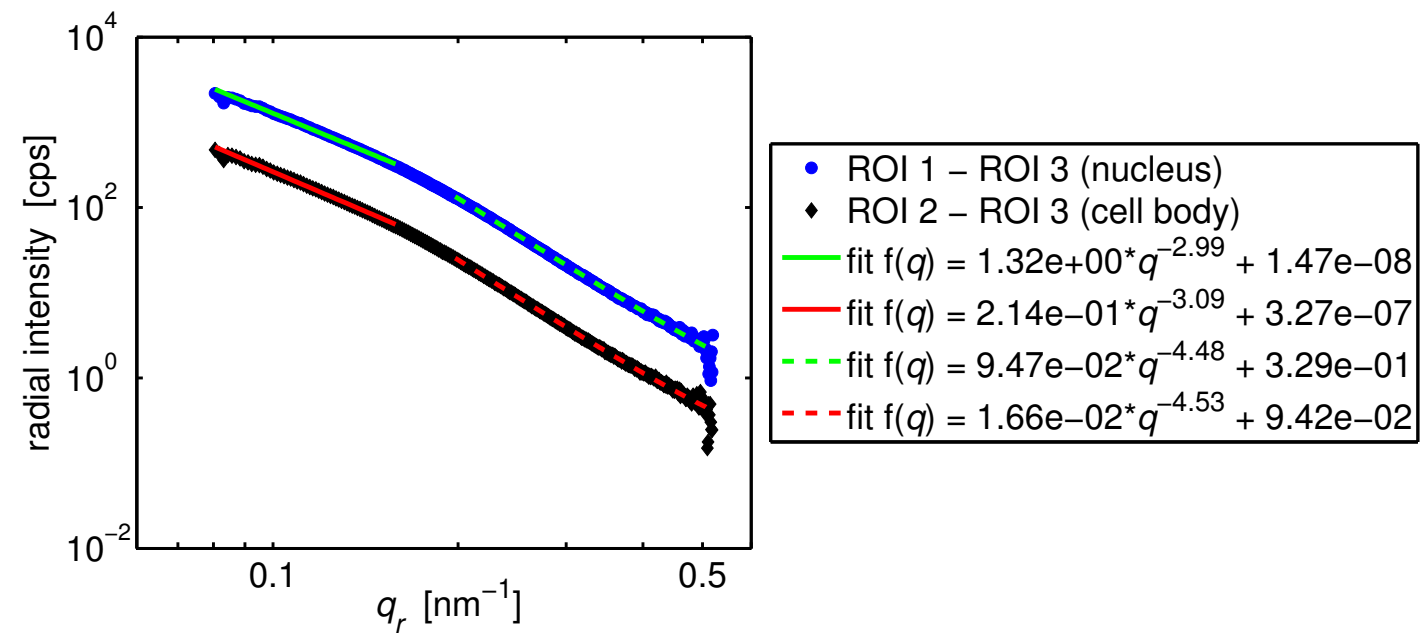

Fig. 5.20.: Fitting of two power laws to the low $q_{r}$-region and high $q_{r}$-region of the background corrected average radial intensity profiles, respectively. The $q_{r}$-ranges over which the fit functions are plotted correspond to the regions that were used for fitting. (Sample BW129, position 3; P10, Oct. 2012) 


\subsubsection{Comparison of Power Law Exponents}

The same analysis scheme as presented above was applied to six cells that were measured at the P10 beamline, four of which with a keratin network and two without keratin. The X-ray dark-field image as well as the corresponding visible light phase contrast and fluorescence microscopy images as well as the background corrected radial intensity profiles along with the power law fits for these measurements are shown in appendix B.1. An overview of the obtained power law exponents is depicted in Fig. 5.21. For each group of data points, the first column corresponds to cells with a keratin network and the second column to cells without a keratin network.

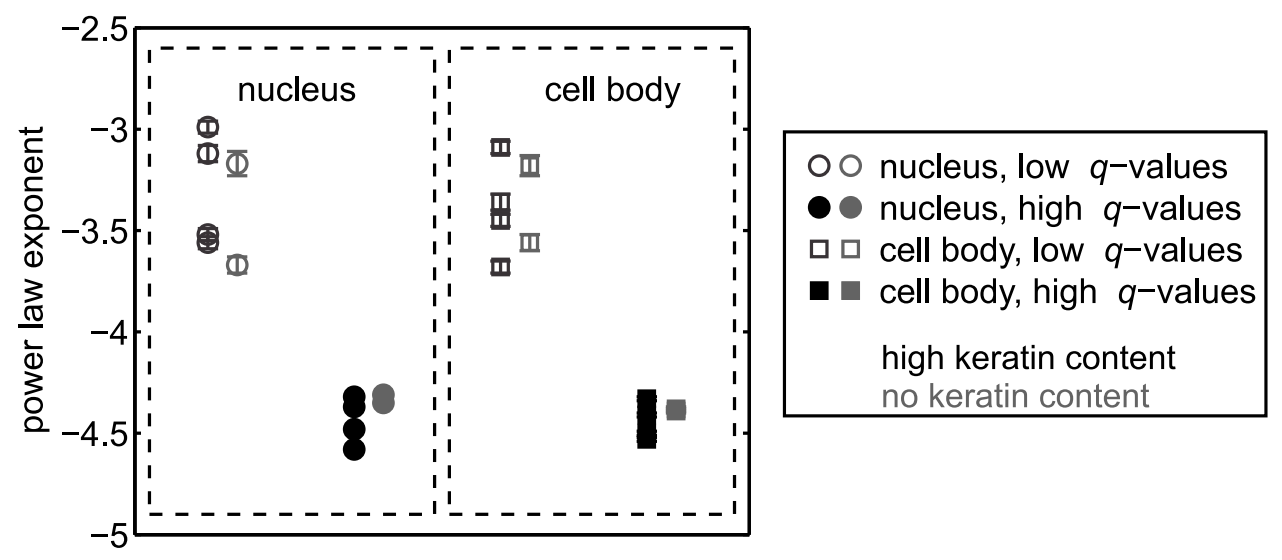

Fig. 5.21.: Comparison of the power law exponents for freeze-dried cells with and without a keratin network measured at the P10 beamline. For each group of data points, the first column corresponds to cells with a keratin network and the second column to cells without a keratin network. The error bars indicate the errors obtained from the fit. The data were taken at P10, Oct. 2012 and March 2013.

All power law exponents obtained in the low $q_{r}$ region are in a range of about -3.0 to -3.5 and the power law exponents obtained in the high $q_{r}$ region are in a range of about -4.3 to -4.6 . The power law exponents for all measured freeze-dried cells were averaged taking into account the position on the cell, i.e. the nucleus or the cell body, and the fit region. The resulting mean power law exponents and the standard deviation of the mean presented in Tab. 5.3. For averaging, the "normal" average and not the "weighted" average with regard to the error was used, because the errors only include the goodness of the fits and no information about the accuracy of the measurement or the state of the measured cell. No difference in the power law exponents is observed between the cells with or without a keratin network. Therefore either cells with a higher portion of keratin filament or bundles in the cytoplasm are needed to distinguish between keratin-free and keratin-containing cells on the level of the intensity decay of the averaged scattering signal, or the (averaged) scattering 


\begin{tabular}{lc}
\hline & mean power law exponent \\
\hline nucleus, low $q_{r}$-values & $-3.34 \pm 0.12$ \\
nucleus, high $q_{r}$-values & $-4.40 \pm 0.05$ \\
cell body, low $q_{r}$-values & $-3.39 \pm 0.10$ \\
cell body, high $q_{r}$-values & $-4.42 \pm 0.04$ \\
\hline
\end{tabular}

Tab. 5.3.: Mean power law exponents and standard deviation of the mean for all freezedried SK8/18-2 cells that were measured at the P10 beamline. (P10, Oct. 2012 and March 2013)

signals from different structures in the cell are too similar to be distinguished at all. The power law exponents obtained on the nuclear region and on the cell body are also identical within the error range, even though the composition and structure of these two regions, and therefore also the scattering signal, should be different. Therefore the average structure of the cellular material in freeze-dried SK8/18-2 cells seems to be very similar for different cellular compartments like the nucleus and the cytoplasm and thereby also similar averaged scattering signals are obtained.

\subsubsection{Radial Intensities of Single Scattering Patterns}

For the measurements on freeze-dried cells taken at the P10 beamline, single scattering patterns show a strong scattering signal. Examples for single scattering patterns from four different positions, i.e., the nucleus, the cell body, the cellular extension at the lower part of the cell and an empty position, are presented in Fig. $5.22 \mathrm{a}-\mathrm{d}$. The positions of the scattering patterns in the scan are given above the scattering patterns and the positions are also marked in the X-ray dark-field image from this scan in Fig. 5.22 e. In contrast to the scattering patterns recorded at the ID13 beamline, no scattering maxima or minima are visible in the scattering patterns recorded at the P10 beamline. The anisotropy of the scattering pattern recorded on the cellular extension in Fig. 5.22 $\mathrm{r}$ is not as pronounced as it was for the data recorded at the ID13 beamline.

Each scattering pattern was integrated in azimuthal direction in one segment yielding the radial intensity profiles in Fig. 5.22f. For comparison also the radial intensity profile of the averaged scattering pattern from the empty region is plotted in the same figure. The radial intensity profiles from the cell show a strong signal, which is well above the radial intensity of the single and averaged scattering patterns from the empty region. The radial intensity profiles from all positions on the cell show a smooth decay without peaks or shoulders. 

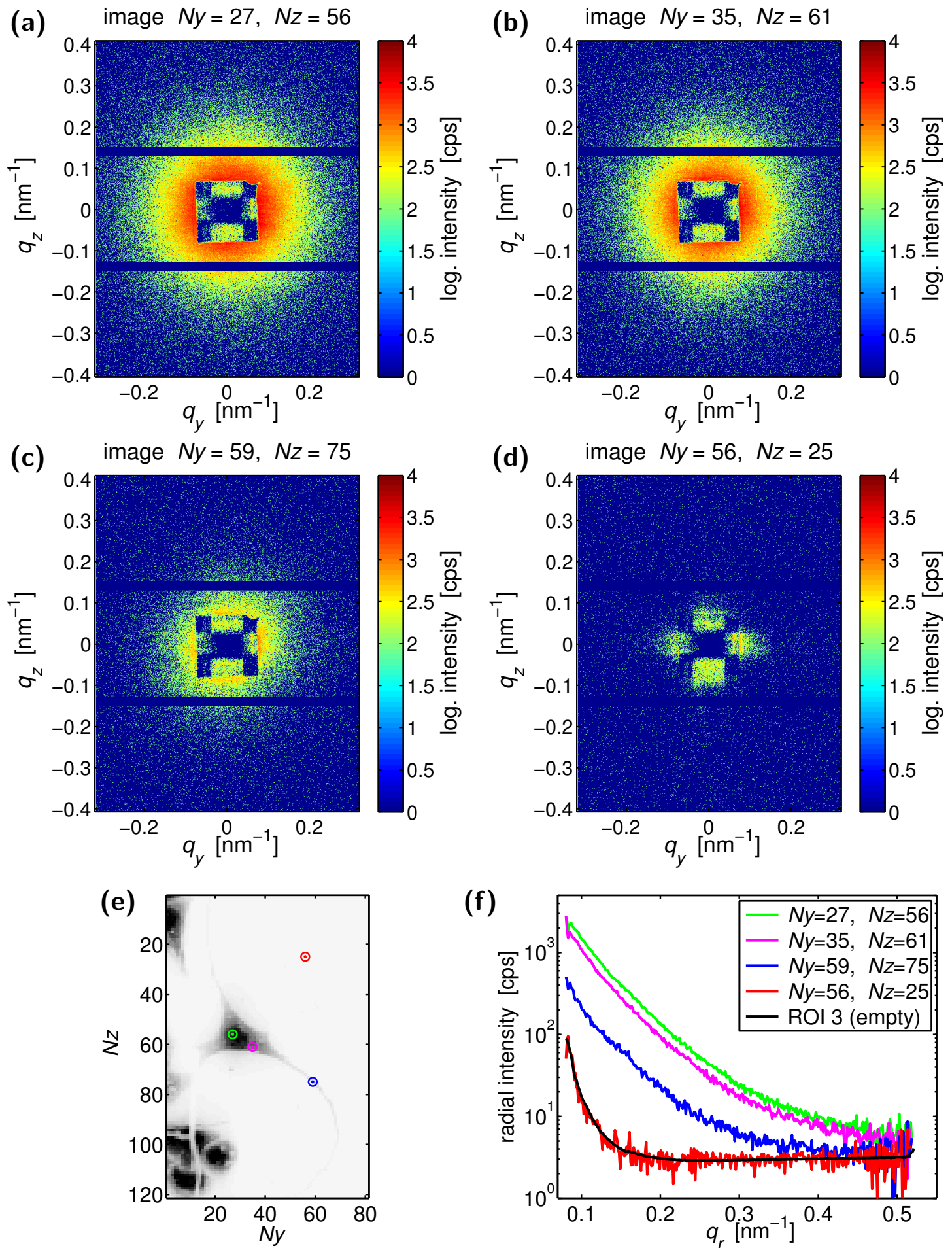

Fig. 5.22.: Single scattering pattern from four different scan positions, i.e. (a) the nucleus, (b) the cell body, (c) the cellular extension and (d) an empty position. (e) Scan positions from which the four scattering patterns were selected. (f) Radial intensity profiles of the four scattering patterns along with the averaged background signal, which was determined above. (Sample BW129, position 3; P10, Oct. 2012) 


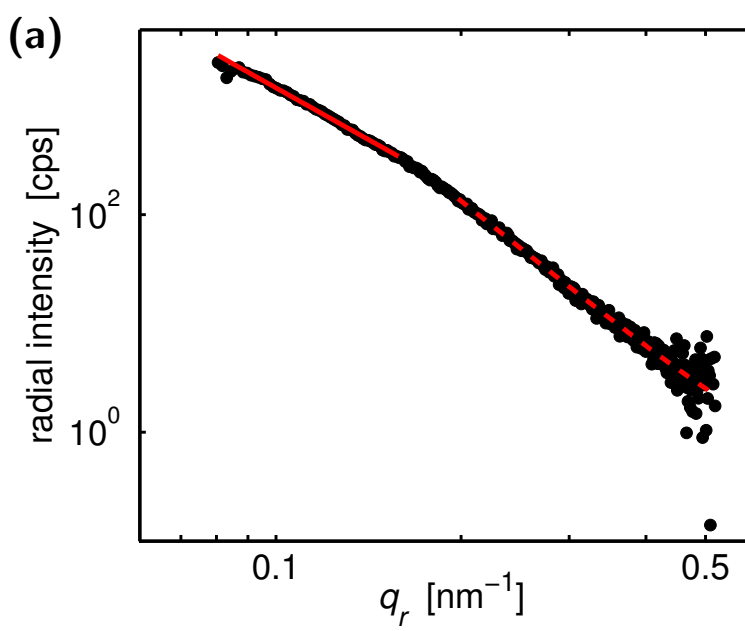

$N y=27, N z=56$, nucleus

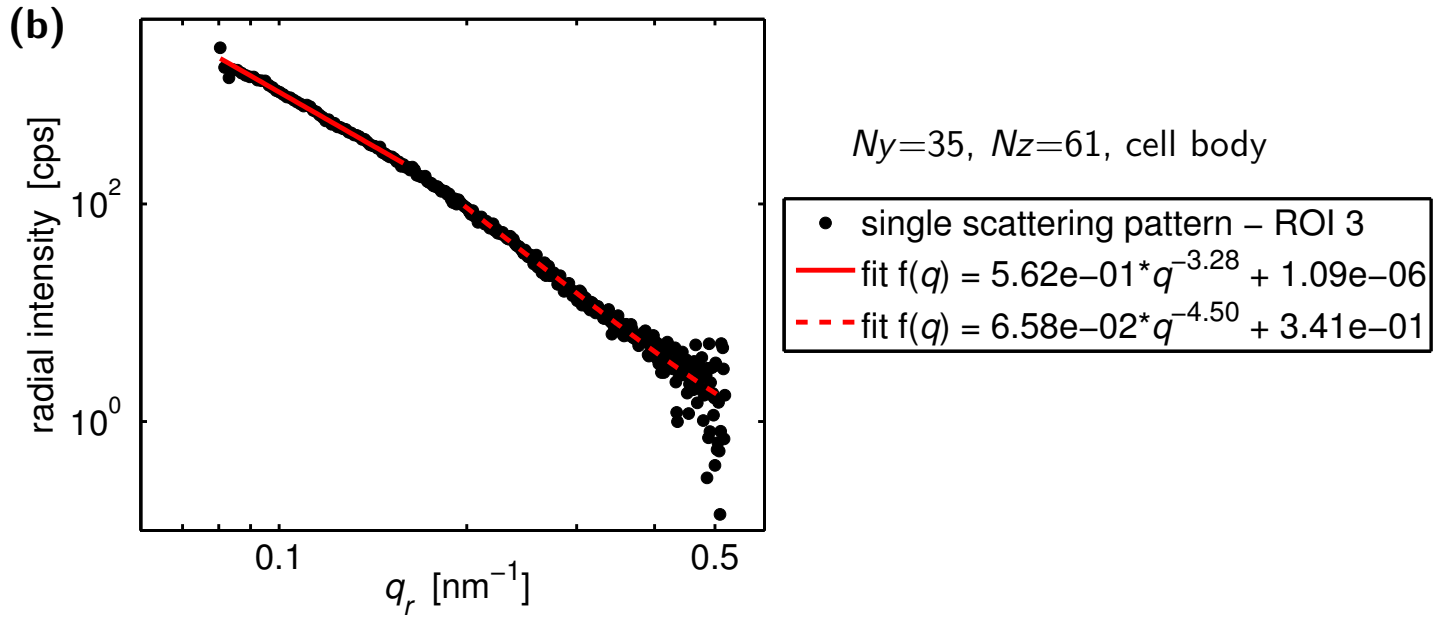

- single scattering pattern - $\mathrm{ROI} 3$

— fit $f(q)=1.01 e+00^{*} q^{-3.16}+3.19 e-09$

- - - fit $f(q)=9.36 e-02^{*} q^{-4.51}+3.94 e-01$

(b)

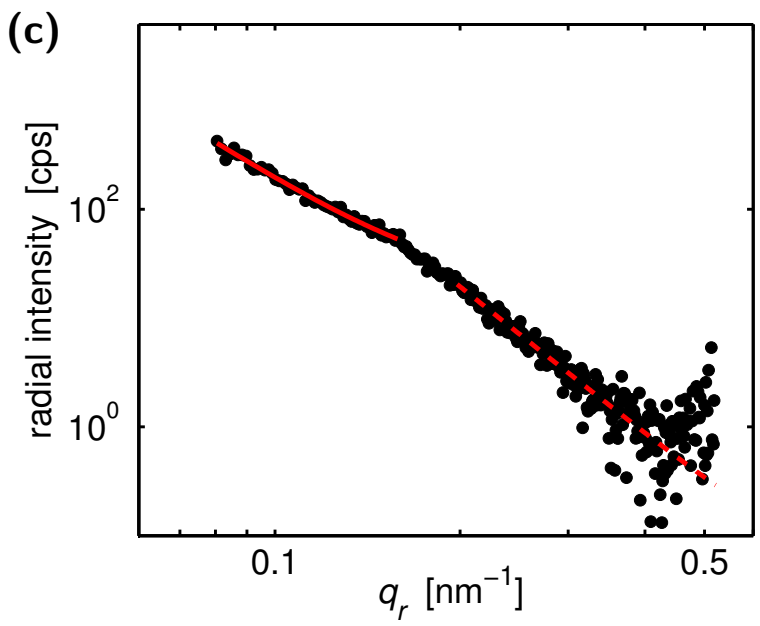

$N y=59, N z=75$, cellular extension

- single scattering pattern - $\mathrm{ROI} 3$

- fit $\mathrm{f}(q)=4.74 \mathrm{e}-02^{*} q^{-3.57}+1.89 \mathrm{e}+01$

- - - fit $f(q)=1.34 \mathrm{e}-02^{*} q^{-4.52}+3.56 \mathrm{e}-02$

Fig. 5.23.: Fitting of two power laws to the low $q_{r}$-region and high $q_{r}$-region, respectively, of the background corrected radial intensity profiles of single scattering patterns from (a) the nucleus, (b) the cell body and (c) the cellular extension. The $q_{r}$-ranges over which the fit functions are plotted correspond to the regions that were used for fitting. (Sample BW129, position 3; P10, Oct. 2012) 
Subtraction of the averaged background signal (ROI 3 in Fig. 5.22f) from the radial intensity profiles at the different positions on the cell results in the curves shown in Fig. 5.23. In analogy to the analysis of radial intensity profiles of averaged scattering patterns, the radial intensity profiles of the single scattering patterns were fitted in the low and high $q_{r}$-region with two power law function, respectively. The fits are displayed in 5.23 along with the background corrected radial intensity profiles. The obtained power law exponents are in the same ranges as the ones for the average radial intensity profiles.

The fact that the radial intensity profiles of single scattering patterns show a smooth decay and no peaks is probably related to two experimental factors. Firstly, the beam size at the P10 beamline was larger compared to the measurements at the ID13 beamline and thereby larger volumes are probed and averaged in a single scattering pattern. Secondly, at the P10 beamline continuous mesh scans, where the motor of the fast scan axis moves continuously, were employed in order to reduce the total scan time. Therefore not only a sample region in the order of the beam size $(\approx 300 \mathrm{~nm})$ was probed in a single scattering pattern, but a sample region in the order of the step size (here $2 \mu \mathrm{m}$ ). For continuous mesh scans with a smaller step size of $500 \mathrm{~nm}$, the scattering patterns do not show peaks in the signal either (compare Fig. B.5 in the appendix), which indicates that either normal (not continuous) mesh scans need to be used in order to obtain maxima or minima in the scattering signal or a small beam must be employed. A single scattering pattern taken at the P10 beamline therefore corresponds to an average of (at least) $2 \times 2$ neighboring scattering patterns taken at the ID13 beamline, in which scattering peaks are also not present.

The scattering pattern recorded on the cellular extension was integrated in eight angular segments to test whether the segments differ and whether peaks or shoulders can be observed in the signal from single segments. Fig. 5.23 a shows the scattering pattern from the cellular extension along with dashed white lines indicating the angular segments used for azimuthal integration. The radial intensity profiles after background subtraction are displayed in Fig. $5.23 \mathrm{~b}$. For the background signal, the averaged scattering pattern from the empty region in Fig. 5.18 was used. The eight radial intensity profiles show a smooth intensity decay as it was also observed for integration in one angular segment. Small bumps in the profiles, e.g. in segment number 2 (green curve) at around $0.2 \mathrm{~nm}^{-1}$, can be explained by locally higher noise in the radial intensity due to the non-sensitive pixel lines between two detector modules and therefore the lower number of available pixels for averaging. Hence, information about the local substructure in the cellular samples cannot be extracted from the scattering pattern presented in this section. 


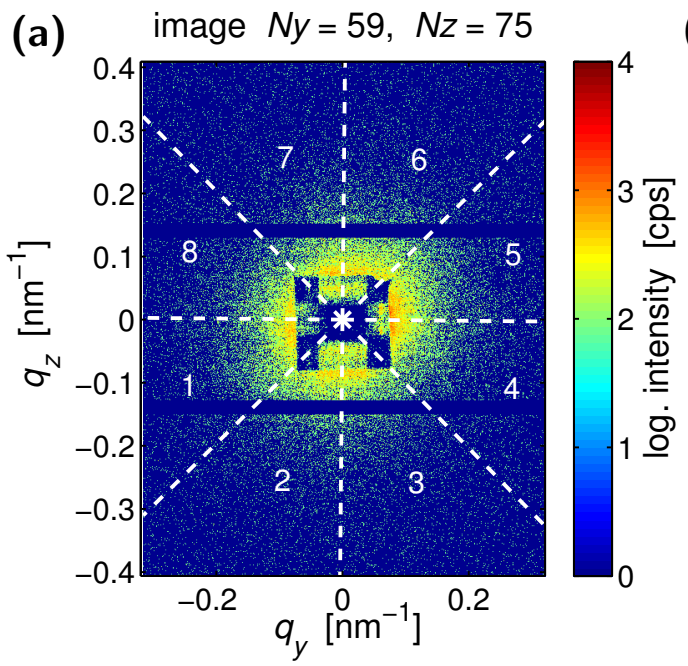

(b)

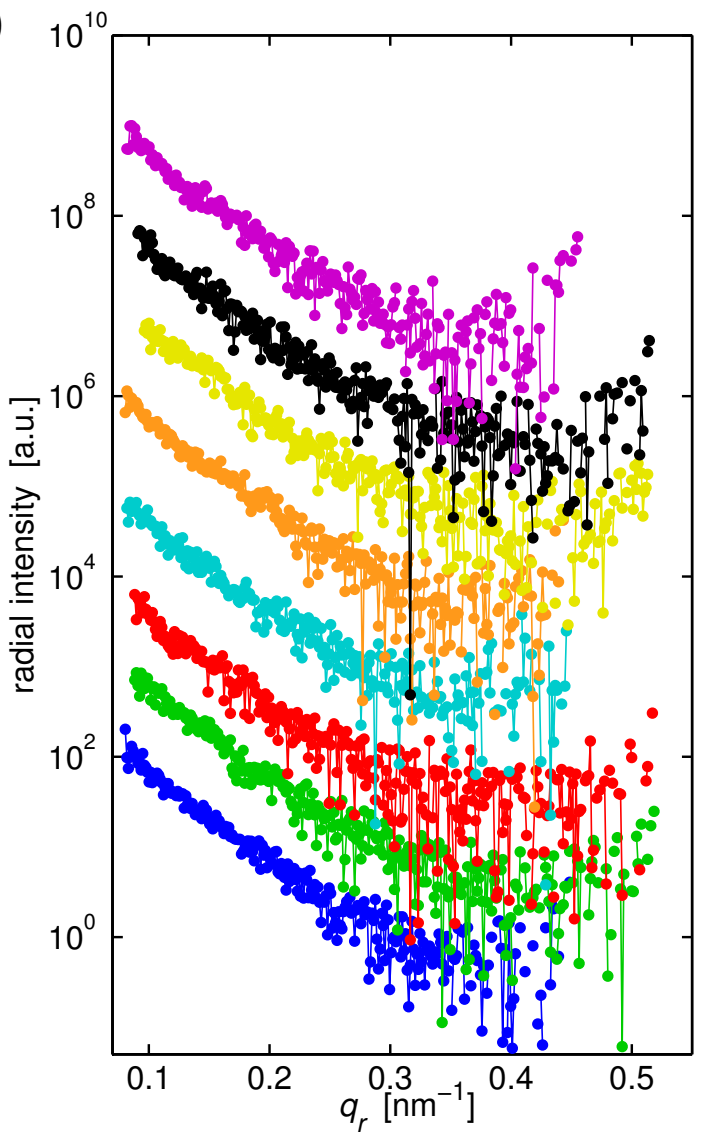

Fig. 5.24.: Azimuthal integration of a scattering pattern from the cellular extension in eight angular segments. (a) Scattering pattern from the cellular extension along with white lines indicating the eight angular segments used for azimuthal integration. (b) Radial intensity profiles after background subtraction. The radial intensity profiles are shifted upwards for clarity. (Sample BW129, position 3; P10, Oct. 2012) 


\subsection{Results from Measurements at the cSAXS Beamline}

At the cSAXS beamline, experiments were performed on freeze-dried samples that were treated with the IF lysis buffer as well as on samples without this treatment. Furthermore, cells with a keratin network and keratin-free cells were measured. However, since the results from measurements at the P10 beamline and the ID13 beamline as presented above, do not show distinct differences between cells with or without a keratin network and since the only difference between the cells that were treated with the IF lysis buffer and untreated cells is a lower or higher scattering intensity, only one example for a measurement on a untreated cell with a keratin network is presented here. An overview of scan parameters and the estimated average radiation doses per step for this measurement is shown in Tab 5.4

\begin{tabular}{lllrlll}
\hline sample & set-up & \multicolumn{1}{c}{$\Delta_{x, y}\left[\mu \mathrm{m}^{2}\right]$} & $N_{x} \times N_{y}$ & $T[\mathrm{~s}]$ & $T_{\text {att }}$ & $D[\mathrm{~Gy}]$ \\
\hline BW203, pos. 2A & Feb. 2013 & $1 \times 1$ & $81 \times 81$ & 0.05 & 0.122 & $1.8 \times 10^{3}$ \\
BW203, pos. 2B & Feb. 2013 & $0.1 \times 0.1$ & $101 \times 51$ & 1 & 1 & $3.0 \times 10^{7}$ \\
\hline
\end{tabular}

Tab. 5.4.: Summary of the scan parameters and the estimated average radiation doses for the measurements on freeze-dried cells at the cSAXS beamline. Here, $\Delta_{x, y}$ are the lateral step sizes, $N_{x}$ and $N_{y}$ are the number of scan points in $x$ - and $y$-direction, $T$ is the exposure time, $T_{\text {att }}$ the attenuator transmission and $D$ the estimated average radiation dose per step.

\subsubsection{X-Ray Dark-Field Images and Structure Orientation}

For the reconstruction of the X-ray dark-field images taken at the cSAXS beamline, the Matlab-based analysis script stxm_online.m was used, which was developed at the cSAXS beamline and kindly provided [91, 136]. Fig. 5.25a and b show the fluorescence microscopy image of the keratin network in a group of SK8/18-2 cells taken before plunge-freezing and freeze-drying and the visible light phase contrast microscopy image after freeze-drying. In the cells at the bottom of the image, a crack that occurred during plunge-freezing or freeze-drying can be identified. However, the cell in the upper part of the image is intact at the resolution of the microscopy image. The X-ray dark-field image from a coarse mesh scan on the same sample region is presented in Fig. 5.25. Despite the low signal-to-noise ratio, the cell contours can be identified in the dark-field image, thereby allowing for the positioning of finer 

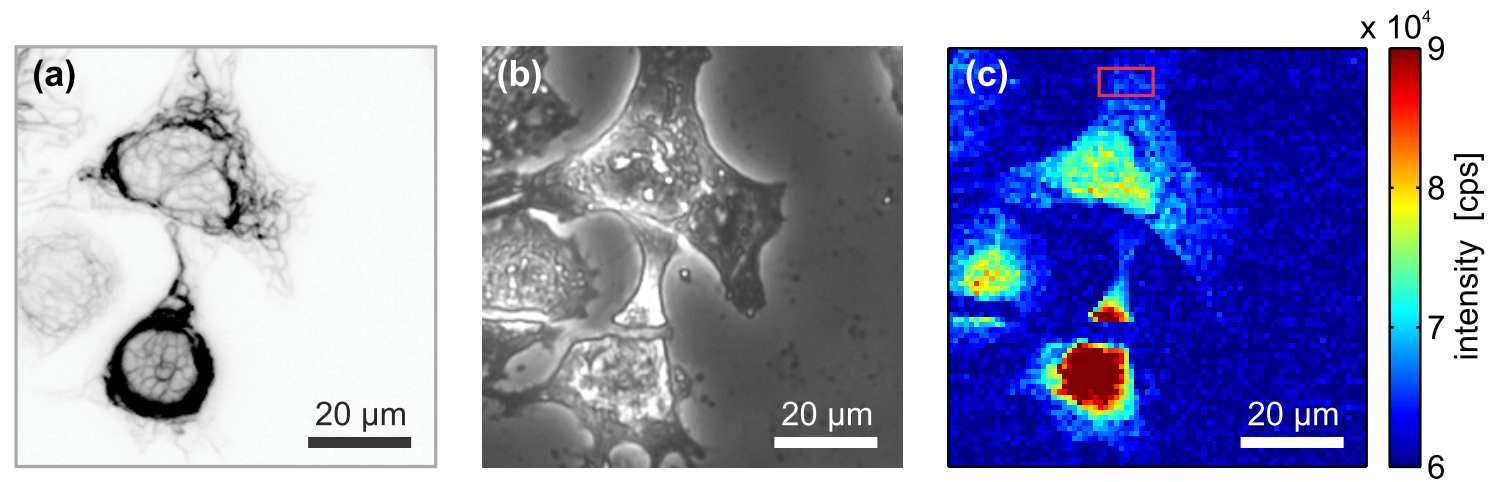

Fig. 5.25.: Microscopy images and X-ray dark-field image of freeze-dried SK8/18-2 cells. (a) Fluorescence microscopy image of the keratin network in the hydrated cells taken before plunge-freezing and freeze-drying and (b) phase contrast microscopy image after freeze-drying. (c) X-ray dark-field image from a scan with a step size of $1 \mu \mathrm{m}$ and an exposure time of $0.05 \mathrm{~s}$ on the same sample region. The red box indicates the position of a region of interest scan. (Sample BW203, position 2A; cSAXS, Feb. 2013)

region of interest scans.

A fine region of interest scan with a step size of $100 \mathrm{~nm}$ and an exposure time of $1 \mathrm{~s}$ was performed on the marked region in Fig. 5.25k. The X-ray dark-field image of this measurement along with the map of the local structure orientation is presented in Fig. 5.26, The local structure orientation indicated by the black lines agrees well with the orientation of the elongated structures in the X-ray dark-field image. However, no thick keratin bundles in the fluorescence microscopy image can be

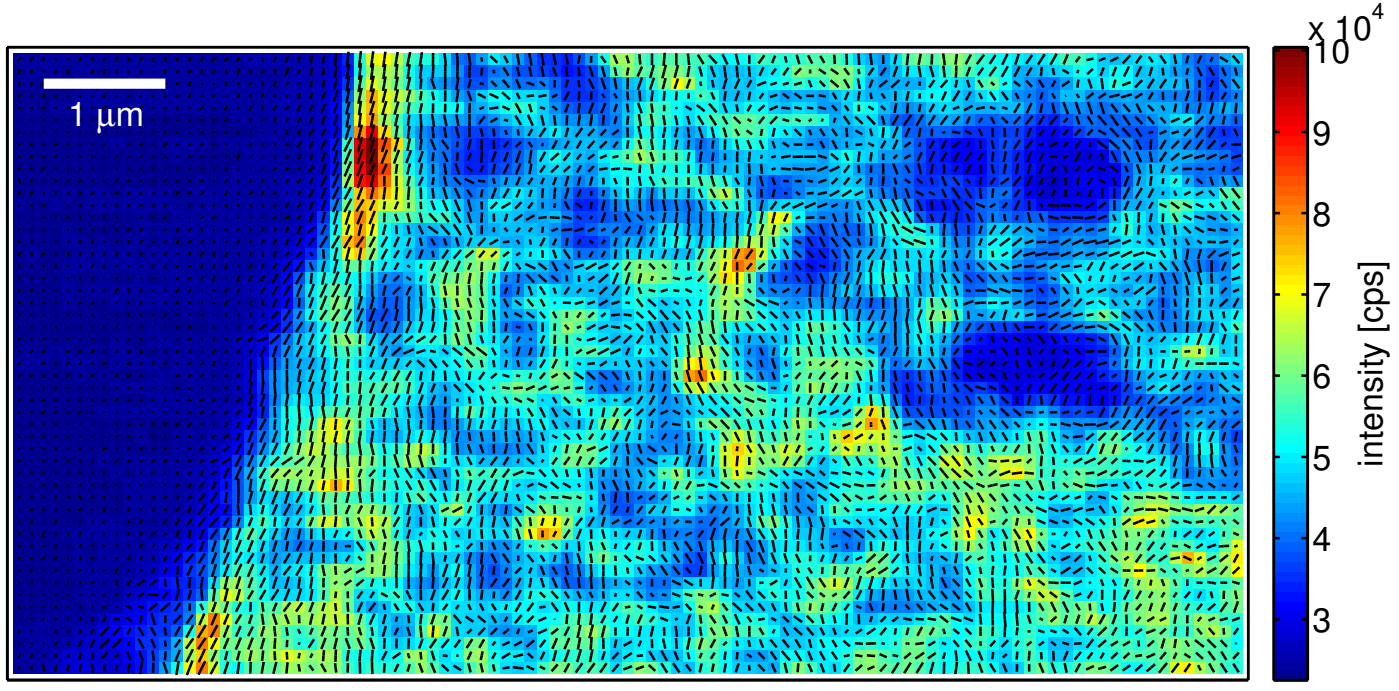

Fig. 5.26.: Structure orientation map as overlay with the X-ray dark-field image from a scan with $100 \mathrm{~nm}$ step size and $1 \mathrm{~s}$ exposure time on the cellular extension in the region that is marked in Fig. 5.25. (Sample BW203, position 2B; cSAXS, Feb. 2013) 
identified in the dark-field image.

\subsubsection{Radial Intensities of Averaged and Single Scattering Patterns}

Scattering patterns from the cellular extension and the empty region in Fig. 5.26 were averaged yielding the scattering patterns in Fig. $5.27 \mathrm{a}$ and b, respectively. In the center, the primary beam is blocked by a beamstop and the holder of the beam-
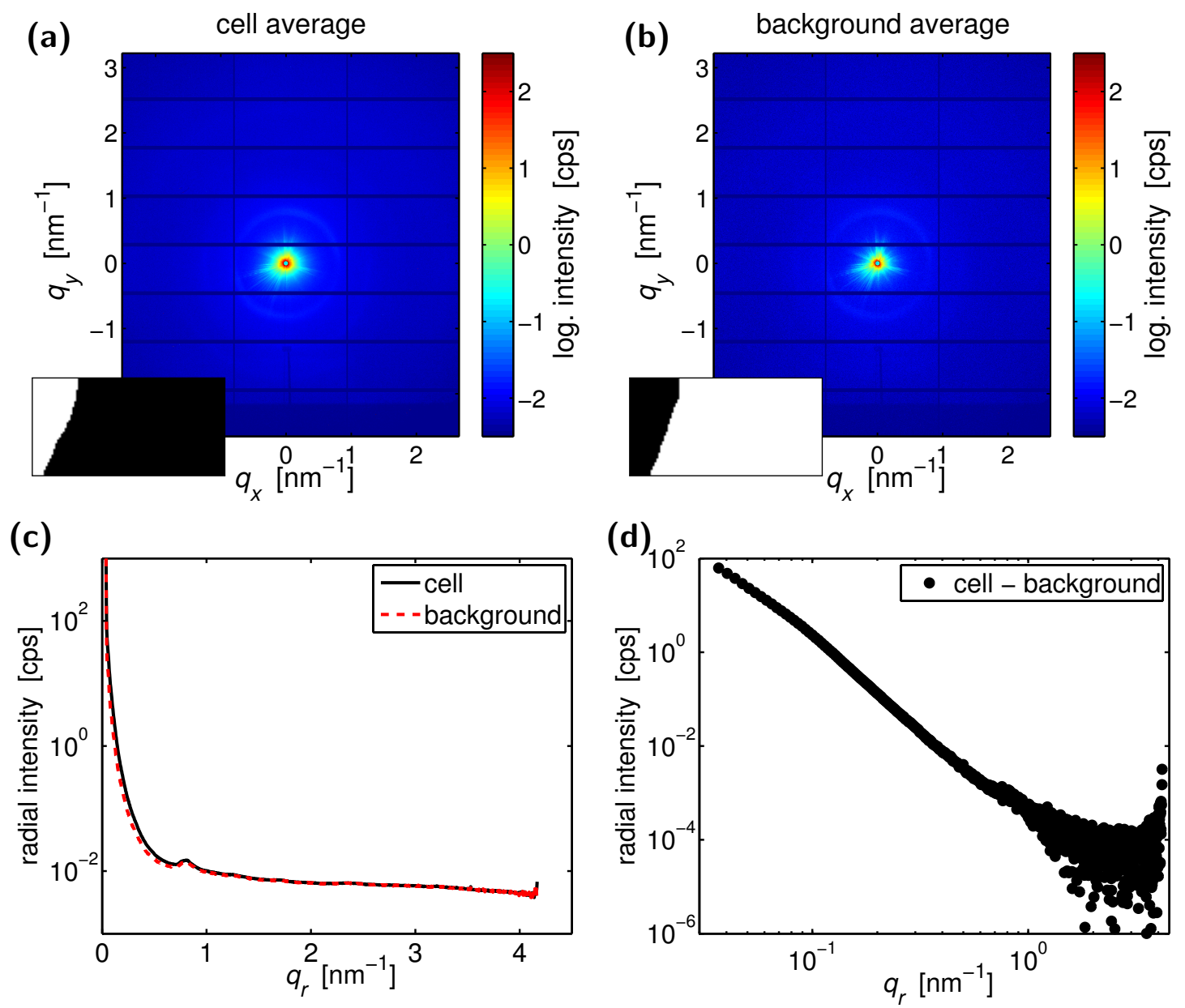

Fig. 5.27.: Azimuthal integration of averaged scattering patterns. (a) Averaged scattering pattern from the cellular extension marked by a red box in Fig. 5.25. (b) Averaged scattering pattern from the empty region. The insets in (a) and (b) show from which regions of the scan (black pixels) the scattering patterns are used to obtain an average scattering pattern from the cellular extension and the empty region. The size of the inset is $100 \times 50 \mu \mathrm{m}^{2}$ (c) Radial intensity profiles from the cell and the background region. (d) Background corrected radial intensity profile. (Sample BW203, position 2B; cSAXS, Feb. 2013) 
stop can be identified as well. Furthermore, the intensity is shielded by the flight tube at the bottom of the scattering pattern. The ring-like scattering around the primary beam corresponds probably to scattering from the window material (Kapton) of the flight tube. Strong scattering from the order sorting aperture is present around the primary beam. The averaged scattering patterns were integrated in azimuthal direction resulting in the radial intensity profiles shown in Fig. 5.27k. The scattering signal from the cell is slightly stronger than the signal from the background region. Subtraction of the background signal from the cell signal results in the radial intensity profile presented in Fig. 5.27d. Despite the small difference between the radial intensity profiles from the cell region and the empty region, the background corrected radial intensity shows a low noise level up to about $1 \mathrm{~nm}^{-1}$. Furthermore the radial intensity profile displays a kink at around $0.1 \mathrm{~nm}^{-1}$.

The background corrected radial intensity was fitted with two power law functions in the low $q_{r}$-region and high $q_{r}$-region yielding power law exponents of -3.30 and -4.10 , respectively, which agrees well with the results from measurements recorded at the P10 beamline. The fit functions are shown in Fig. 5.28 along with the data. The radial intensity is well approximated by the fit functions over the respective regions.

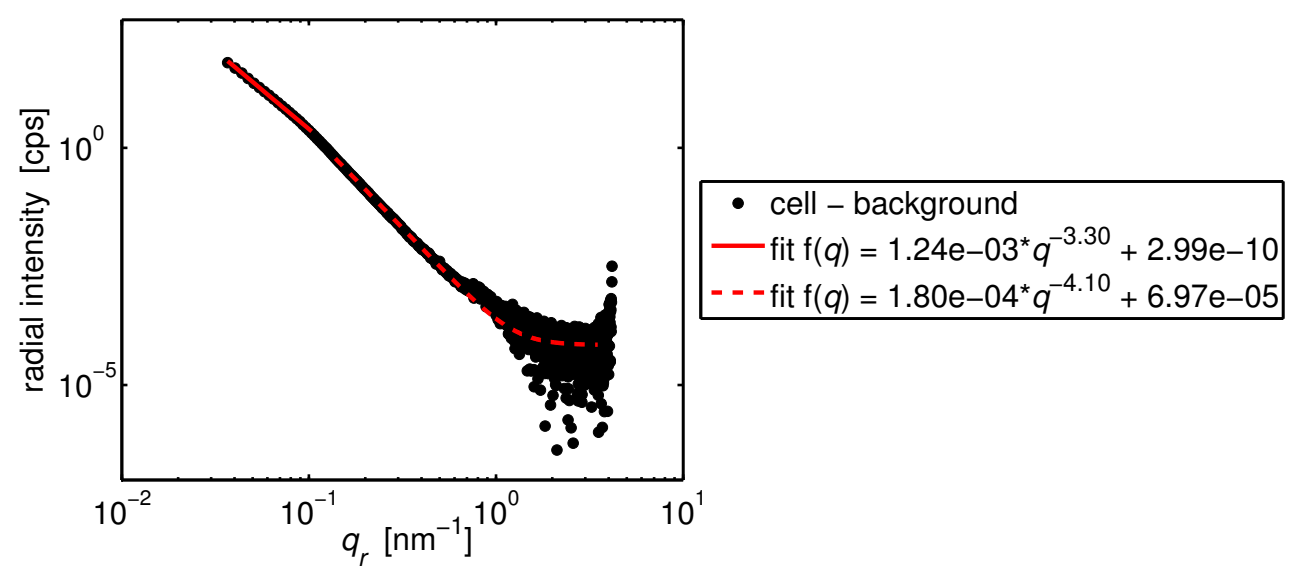

Fig. 5.28.: Fitting of two power laws to the low $q_{r}$-region and high $q_{r}$-region, respectively, of the background corrected radial intensity profile of an averaged scattering pattern from the cellular extension. The $q_{r}$-ranges over which the fit functions are plotted correspond to the regions that were used for fitting. (Sample BW203, position 2B; cSAXS, Feb. 2013)

For single scattering patterns recorded at the cSAXS beamline, the background scattering was so strong that the signal from the cell cannot be identified without background subtraction. However, after subtraction of the averaged background signal, which was recorded on an empty region, from the scattering signal recorded on the cell, the locally anisotropic scattering signal from the cell can be identified, as 

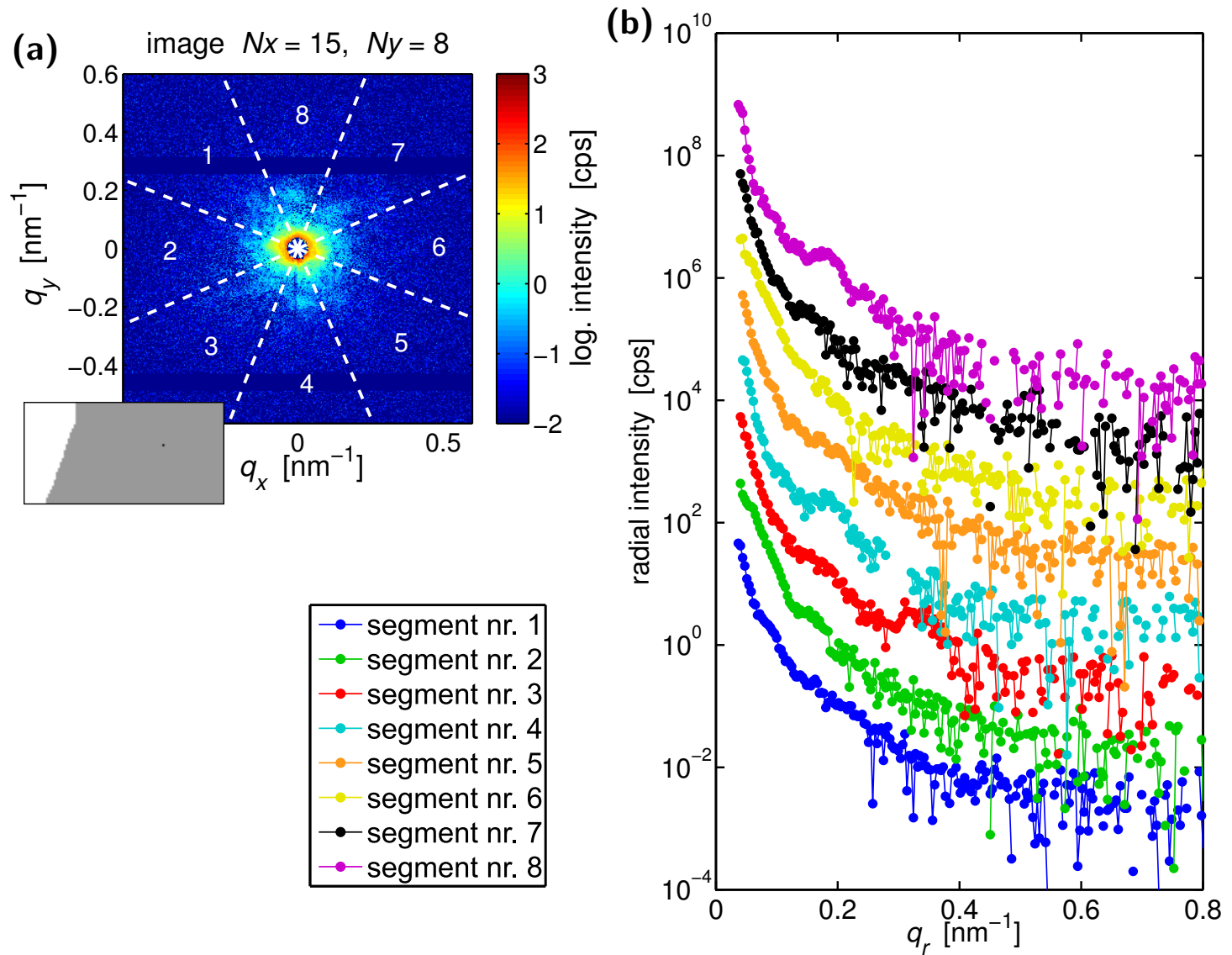

Fig. 5.29.: Azimuthal integration of a scattering pattern from the cellular extension in eight angular segments. (a) Scattering pattern from the cellular extension after subtraction of the averaged background signal along with white lines indicating the eight angular segments. The inset shows which scattering pattern of the scan (black pixel) was used. (b) Radial intensity profiles after background subtraction. The radial intensity profiles are shifted upwards for clarity. (Sample BW203, position 2B; cSAXS, Feb. 2013)

presented in Fig. $5.29 \mathrm{a}$ for an example scattering pattern. In the scattering pattern regions with higher or lower intensity can be identified.

The scattering pattern was divided into eight angular segments such that the first and fifth segment were centered around the direction of strongest scattering, and it was integrated in azimuthal direction. The corresponding radial intensity profiles are presented in Fig. $5.29 \mathrm{~b}$. In the radial intensity profiles, local intensity maxima can be identified at around $0.18 \mathrm{~nm}^{-1}$ and $0.34 \mathrm{~nm}^{-1}$. At higher $q_{r}$-values, the signal-to-noise ratio is too low to identify further intensity maxima or minima. To increase the signal-to-noise ratio in the scattering pattern, $3 \times 3$ scattering patterns, which were centered around the scattering patterns shown in Fig. $5.29 \mathrm{a}$, were averaged yielding the scattering pattern shown in Fig. 5.30a. In analogy to the analysis of the single scattering patterns, the averaged scattering pattern was 

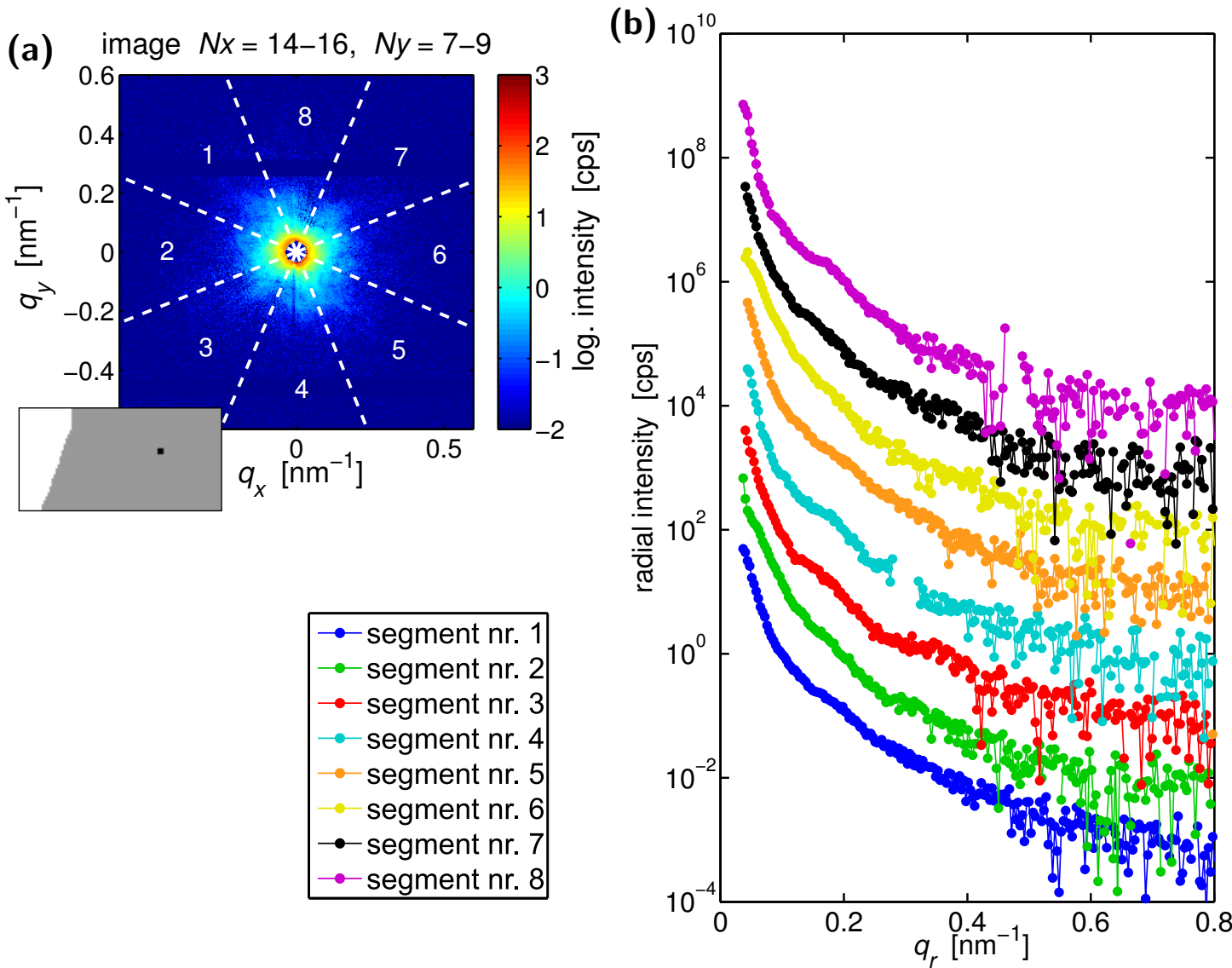

Fig. 5.30.: Azimuthal integration of an average of $3 \times 3$ scattering patterns from the cellular extension in eight angular segments. (a) Scattering pattern from the cellular extension after subtraction of the averaged background signal along with white lines indicating the eight angular segments. The inset shows which scattering patterns of the scan (black pixels) were averaged. (b) Radial intensity profiles after background subtraction. The radial intensity profiles are shifted upwards for clarity. (Sample BW203, position 2B; cSAXS, Feb. 2013)

integrated in eight angular segments, yielding the radial intensity profiles in Fig. 5.30 p. The radial intensity profiles are less noisy and also exhibit local intensity maxima or shoulders at around $0.18 \mathrm{~nm}^{-1}$ and $0.34 \mathrm{~nm}^{-1}$. Further intensity maxima or minima at higher $q_{r}$-values cannot be identified. However, similar to the analysis of the single scattering patterns recorded at the ID13 beamline, a smoothing of the intensity maxima or shoulders in the intensity decay is observed, which indicates a high structural heterogeneity in the cellular sample. 


\subsection{Summary and Closing Discussion}

Even though biological samples are generally weak scatterers, X-ray dark-field images of freeze-dried SK8/18-2 cells could be recorded at each of the three set-ups. The cells, the nuclei and substructures in the cell body and in the nuclei could be clearly identified in scans on large sample areas, allowing for a precise positioning of finer region of interest scan. For cells that were treated with IF lysis buffer, the scattering signal in the X-ray dark-field images was much weaker. As described in section 4.2.5, treatment of the cells with IF lysis buffer leads to the depolymerization of actin filaments and microtubules, and the removal of all soluble components from the cytosol. Therefore, the observation that the treated cells scatter less than the untreated cells agrees with the fact that less protein material is present in these cells. Since the treated samples were not fluorescently stained for filamentous actin or microtubules to validate the effect of IF lysis buffer for each cell, remaining actin filaments or microtubules in individual cells cannot be totally excluded. In this context it should be also mentioned that it is not possible to stain filamentous actin and microtubules simultaneously in the same SK8/18-2 cell, due to the available dyes and the already fluorescently label keratin network. Furthermore, the effect of IF lysis buffer on other cellular organelles was not tested. Substructures in the cytoplasm that were visible using bright-field microscopy (compare Fig. 4.7) indicated that not all cellular organelles are removed from the cell during the treatment. In this context, other protocols for the extraction of the keratin network that have been employed for electron microscopy studies on the pure network [69, 70], should be tested in combination with scanning X-ray diffraction experiments.

The resolution of the X-ray dark-field images in real space was limited by the beam size used for scanning, i.e. at minimum to about $100 \mathrm{~nm}$. The obtained resolution is therefore well below the resolution achieved by other techniques based on X-rays as a probe like ptychographic or holographic imaging [10, 12, 95, 137]. However, only for scanning X-ray diffraction with a nanometer sized beam and the sample placed in the focus, a small sample volume is probed by recording a full scattering pattern at each scan position, which can then be precisely correlated to the position on the cell using the X-ray dark-field image.

When comparing the X-ray dark-field images to fluorescence microscopy images of the keratin network, similarities between the keratin network morphology and the intensity distribution could be observed. In particular, the elongated structures in the X-ray dark-field image in Fig. 5.5d might correspond to the thick keratin bundles in the fluorescence microscopy image in Fig. 5.5a. By contrast, the Xray dark-field images of keratin-free cells (cf. Fig. 5.11) show elongated structures and clusters, which are larger in size as compared to the keratin-containing cells. A 
network-like intensity distribution is not observed for keratin-free cells. As discussed previously, the morphological differences between the X-ray dark-field image in combination with the orientation map, and the fluorescence microscopy image might be attributed to different factors. The resolution of the fluorescence microscopy images of the keratin network is comparatively low, which does not allow for imaging of thin keratin bundles. Other cytoskeletal structures like microtubules or actin filaments are not visualized in the fluorescence microscopy image, but they might cause a similar scattering signal and their location in the cell overlaps with the keratin network (compare Fig. 2.1 showing the keratin network and filamentous actin in a SK8/18-2 cell). Furthermore, the diffraction experiments are performed on freezedried samples and artifacts from the preparation cannot be excluded. In particular, protein or salt accumulations can be generated during plunge-freezing if (expanding) crystalline ice forms during the freezing process instead of vitreous ice.

In some cells, micrometer-sized ruptures could be identified after freeze-drying. To exclude or confirm further artifacts at the nanometer-scale due to the sample preparation, and here particularly the plunge-freezing process, several different tests should be performed. Firstly, the plunge-frozen sample could be studied at cryogenic conditions with X-ray diffraction to probe the presence of ice crystals. Secondly, high-pressure freezing, which is commonly employed for samples thicker than about $10 \mu \mathrm{m}$, could be applied instead of plunge-freezing [138]. Here, a growth substrate different from $\mathrm{Si}_{3} \mathrm{~N}_{4}$ membrane windows and in particular based on electron microscopy grids, might be necessary due to the constrictions of the sample chamber. Thirdly, critical point drying could be employed for the preparation of dry cellular sample instead of plunge-freezing or high-pressure freezing and consecutive freeze-drying [139].

To determine the local structure orientation and the anisotropy in the sample, we used the approach of approximating the thresholded intensity by an ellipse. The direction of the minor axis in reciprocal space was employed as estimate for the average direction of oriented structures in the sample in real space at the measured position and the eccentricity was employed as a measure for the average degree of orientation. A typical method to determine the anisotropy in the scattering signal is by radial integration of the scattered intensity in a ring in reciprocal space and fitting the obtained azimuthal intensity curve. Here, the amplitude yields a measure for the degree of sample orientation. Further, specific structures with characteristic length scales in the sample can be visualized by selecting a certain region of momentum transfer. Recently this method has been also applied to small angle scattering from biological samples, i.e., a mouse soleus muscle [17 or the dentinal collagen network in human teeth 16, 140,141]. In contrast to these samples with well-defined 
and well-known real space structures, the structure of keratin bundles in our samples is probably not perfectly homogeneous and would therefore lead to scattering in different $q$-regions. Hence, in our case it is important that the analysis allows for the determination of the anisotropy of the complete scattering pattern and not solely of a selected region of momentum transfer in reciprocal space corresponding to a specific range of structure sizes in real space. The method we used worked reliably for the data sets recorded at the ID13 and the cSAXS beamline. For the data sets recorded a the P10 beamline, a strong preferred direction of structure orientation was observed, which is related to the asymmetric beam profile of the KB beam. Fig. 5.31 presents a comparison of typical examples for radial intensity profiles of averaged scattering patterns obtained at the three different beamlines. For better comparability, the same $q_{r}$-range as well as the same number of magnitudes for the intensity axis are used for plotting. The most obvious difference between the data sets recorded at the different beamlines is the much lower difference between the cell signal and the background signal at the cSAXS beamline as compared to the other two beamlines. During the planning and realization of the beamtime at the cSAXS beamline in February 2013, the optimization of the set-up in terms of background reduction was an important issue, but the problem could not be solved with various modification to the set-up (compare section 3.2.3). Furthermore, a kink is visible in the radial intensity profiles recorded at the P10 beamline and at the cSAXS beamline at about $0.1-0.2 \mathrm{~nm}^{-1}$. Therefore, these radial intensity profiles could not be fitted with a single power law over the whole $q_{r}$-range, but two power laws were fitted to the high and low $q_{r}$-region, respectively. A kink was not observed in the radial intensity profiles from averaged scattering patterns recorded at the ID13 beamline, due to the difference in the accessible $q_{r}$-range, which corresponds to the high $q_{r^{-}}$ region measured at the cSAXS beamline and at the P10 beamline. This fact allows for fitting of the whole $q_{r}$-range with a single power law.

A summary of all power law exponents obtained from fits to the radial intensity profiles of averaged scattering patterns recorded on freeze-dried SK8/18-2 cells is shown in Fig. 5.32, Here, the data points obtained at the P10 beamline are depicted in blue, at the cSAXS beamline in red and at the ID13 beamline in green. The measurements at ID13 beamline are assigned to the high $q_{r}$-region. Note that at the cSAXS beamline and at the ID13 beamline region of interest scans on the cellular extensions were employed to obtain the power law exponents, whereas at P10 beamline the whole cell was measured. Therefore at the P10 beamline, the data points are subdivided in the nuclear region and the cell body. The power law exponents obtained at the three different set-ups agree very well. The small deviation of the power law exponents obtained at the cSAXS beamline and at the ID13 beamline 
towards higher values in the high $q_{r}$-region compared to the results obtained at the P10 beamline, might be attributed to the overall lower scattering signal (compare photon counts per second in Fig. 5.31) due to the lower photon flux at these beam-
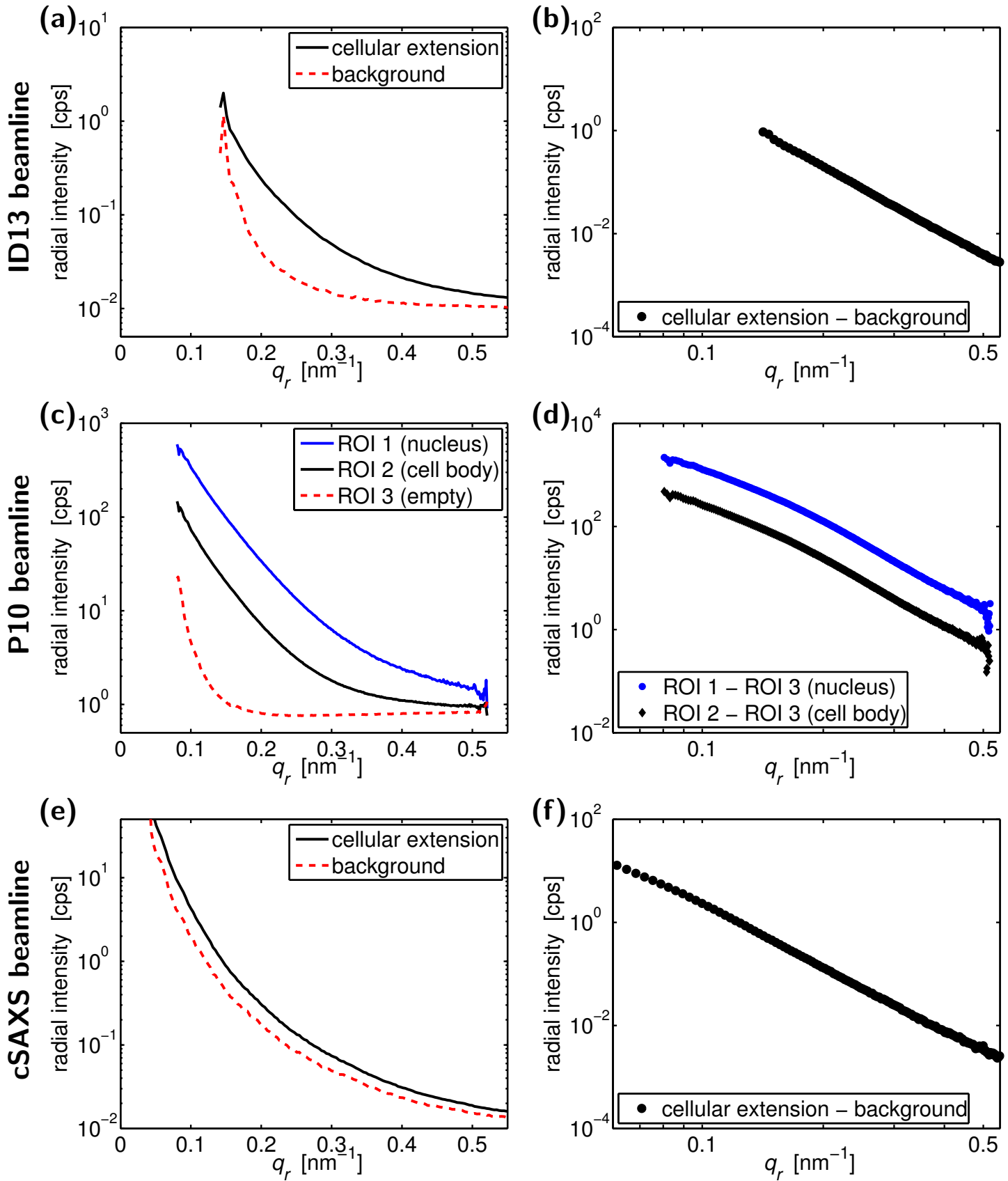

Fig. 5.31.: Comparison of radial intensity profiles of averaged scattering patterns and the corresponding difference curves taken at $(\mathrm{a}, \mathrm{b})$ the ID13 beamline (Sample BW21, position 6C; ID13, Nov. 2011), (c, d) the P10 beamline (Sample BW129, position 3; P10, Oct. 2012) and (e,f) the cSAXS beamline (Sample BW203, position 2B; cSAXS, Feb. 2013). For better comparability, the same $q_{r}$-range as well as the same number of magnitudes for the intensity axis are used for plotting. 
lines and therefore the slightly worse photon statistics.

Similar values of the power law exponents are observed for the cells with or without a keratin network as well as for the cells that were treated with IF lysis buffer. In the high $q_{r}$-region $\left(q_{r} \gtrsim 0.1 \ldots 0.2 \mathrm{~nm}^{-1}\right)$ the power law exponents range from -4.0 to -4.6 and in the low $q_{r}$-region $\left(q_{r} \lesssim 0.1 \ldots 0.2 \mathrm{~nm}^{-1}\right)$ the power law exponents range from -3.0 to -3.7 . Furthermore, no difference is observed between the nuclear region and the cell body. These findings indicate that the averaged structure in the cells, which is probed by the averaged scattering patterns, is very similar for both the different cellular compartments like the nucleus or the cytoplasm as well as for the differently prepared cells, e.g. cells treated with IF lysis buffer, or cells with or without a keratin network. Similar scanning diffraction experiments on freeze-dried bacterial cells yielded exponents ranging from -3 to -4 for the decay of the radial intensity [12]. In this context it would be interesting to perform measurements on different eukaryotic cell lines, e.g. keratinocytes with a high keratin content in the cytoplasm, or on other prokaryotic cells, to investigate whether characteristic differences are obtained.

Corresponding to the well-known Porod law [142, 143], compact two-phase systems with a sharp interface exhibit a power law decay with -4 and further, power law exponents smaller than -4 have been attributed to structures with diffuse boundaries, in which the electron density varies continuously 144, 145]. In our case, the power law exponents are for all samples still close to the classical Porod exponent of

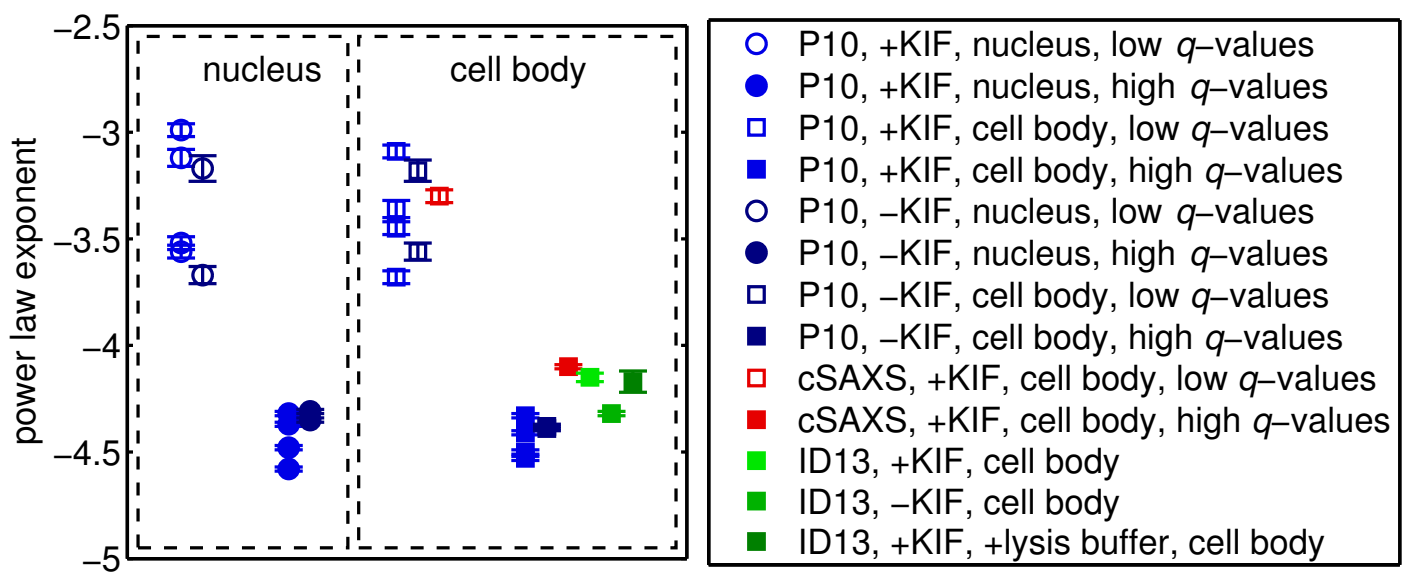

Fig. 5.32.: Summary of all power law exponents obtained from fits to the radial intensity profiles of averaged scattering patterns recorded on freeze-dried SK8/18-2 cells. Data points obtained at the P10 beamline are depicted in blue, at the cSAXS beamline in red and at the ID13 beamline in green. 
-4 , but consistently deviate to smaller numbers in the high $q_{r}$-region and to higher numbers in the low $q_{r}$-region. The deviation might be also caused by the superposition of the scattering signal from various differently shape objects with different sizes in the cell, i.e. various protein, peptides, etc.. Furthermore, the Porod law is commonly used to describe small-angle X-ray scattering (SAXS) experiments, where the employed beam size is large compared to the probed structure sizes. Contrary, the beam size, which we employ for the measurements, is in the same order of magnitude as the structures in the cells. In particular, correlations in the electron density are only probed over an area that is defined by the illumination beam size. To study the effect of the beam size on the obtained power law exponents from freeze-dried cellular samples, measurements with a larger beam in the order of a few micrometers could be performed. The scattering signal from the probed (large) area could then be compared to the averaged scattering signal from several smaller areas, which should be ideally located in the large area on the same sample, probed with a nanometer-sized beam.

The radial intensity profiles from single scattering patterns recorded at the ID13 beamline and at the cSAXS beamline exhibited local intensity maxima and minima, indicating a substructure in the cellular sample. Upon averaging of several adjacent scattering patterns in order to increase the signal-to-noise ratio, the peaks were smoothed or smeared out completely, which hints at a high structural heterogeneity in the sample. However, due to the high heterogeneity of the radial intensity profiles, the strong signal-to-noise ratio in the single scattering patterns and the presumable superposition of different sample structures at each position, it was not possible to automatically fit all profiles in order to determine the underlying structure in the cell. Here, other approaches like a principle component analysis could be employed for the further analysis of single scattering patterns in order to identify the main cellular components that contribute to the scattering signal. The fact that local intensity maxima and minima were not observed in the radial intensity profiles of single scattering patterns recorded at the P10 beamline, can be explained in this context by the larger beam size and the continuous scan mode, which leads to an (averaged) scattering signal from a larger sample area. In this context, experiments at a (nano-focused) free-electron laser (FEL), making use of the higher photon flux and linked to that the potentially higher resolution in reciprocal space, could help to probe and determine the structure or correlation length of the filaments in keratin bundles. For test experiments, the same sample preparation as presented above could be employed and single exposures on well-selected positions in individual cells could be recorded. However, the cells will be (at least locally) destroyed by the intense X-ray beam and therefore the precise localization of certain cellular struc- 
tures in the FEL beam as well as the high sample throughput are critical issues. An automated sample delivery system for adherent cells would be needed.

To get a rough idea of the filament arrangement in keratin bundles in SK8/18-2 cells or in similar cells and the corresponding scattering signal, different experimental approaches would be helpful. Transmission electron microscopy images of the cross-section of a keratin bundle in those cells could show the filament arrangement. However, sectioning might affect the detailed bundle structure. To reduce the complexity of the studied system, in vitro assembled keratin filaments could be investigated using X-ray diffraction. In vitro assembly of keratin filaments as well as the formation of bundles and networks have been described in several publications [46,54 57]. Firstly, the in vitro assembly allows for classical SAXS experiments on the filament bundles in solution kept in capillaries during the data acquisition. Secondly, the bundles or networks could be prepared on silicon nitride membrane windows and could then be studied, either in a hydrated or in a dry state, using scanning diffraction with a nano-focused beam. However, whether the nano-scale structure of the in vitro assembled keratin filaments and bundles resembled the in vivo structures is not known so far. 


\section{Scanning Nano-Diffraction on Fixed-Hydrated Cells}

In this chapter, scanning X-ray nano-diffraction experiments on fixed-hydrated SK8/18-2 cells are described. Fixed-hydrated cells were measured at three different synchrotron radiation facilities and the results are presented and discussed. Furthermore, the capabilities of the different set-ups are compared. Specific challenges that occurred during the measurements fixed-hydrated cells are discussed and solutions are suggested. Parts of this chapter have been prepared for publication [41].

\subsection{Experiment}

The experiments were carried out at three different end-stations at different synchrotron radiation facilities: the cSAXS beamline (SLS), the ID13 beamline (ESRF), and the P10 beamline (PETRA III). The different set-ups and the specific experimental parameters during each beamtime are described separately in section 3.2. For the measurements, cells with a keratin network as well as cells without a keratin network were selected. However, none of the samples was treated with IF lysis buffer, because for the freeze-dried samples the only difference to the untreated cell was a lower scattering signal. First test-experiments on hydrated cells were performed on cells in $\mathrm{Si}_{3} \mathrm{~N}_{4}$ sandwich wet chambers at the cSAXS beamline. The fabrication of these wet chambers is described in section 4.3.1. The wet chambers were attached to an aluminum frame, which was then attached to a magnetic

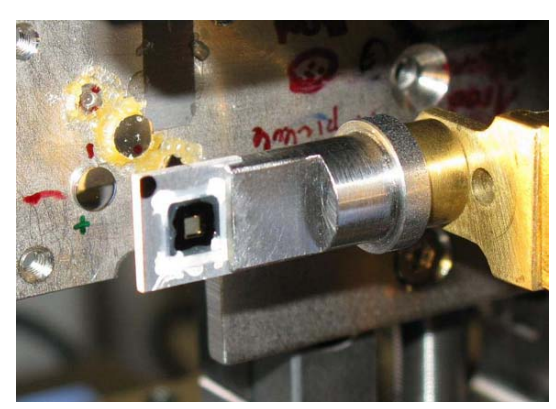

Fig. 6.1.: Photograph of a $\mathrm{Si}_{3} \mathrm{~N}_{4}$ sandwich wet chamber mounted on a magnetic sample holder at the cSAXS beamline (May 2012). sample holder as shown in Fig. 6.1 and mounted at the sample stage.

For the experiments at the ID13 beamline and the P10 beamline, microfluidic devices were employed as sample environment. The microfluidic devices were assem- 
bled directly before the measurement as described in section 4.3.2. Tubings were attached to the inlet and the outlet of the microfluidic device and a Hamilton Gastight

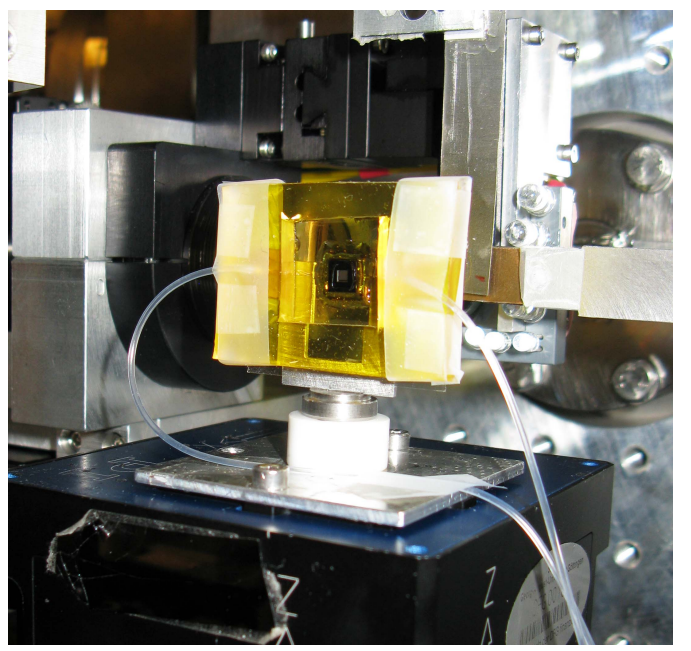

Fig. 6.2.: Photograph of a microfluidic device mounted on a magnetic sample holder at the P10 beamline (March 2013). glass syringe filled with degassed PBS was attached to the inlet tubing allowing for the connection to syringe pumps (neMESYS, Cetoni GmbH, Korbußen, Germany). PBS was pumped through the device at a constant flow rate of 100-200 $\mu \mathrm{l} / \mathrm{h}$, corresponding to a mean flow velocity of about $0.3 \mathrm{~mm} / \mathrm{s}$ depending on the device geometry. Subsequently, the device was mounted on a sample stage using a magnetic sample holder as shown on the photograph in Fig. 6.2. A small waste container for the waste liquid that was flushed through the devices was attached to the sample stage.

All fixed-hydrated samples were mounted with the membrane, on which the cells had been grown, on the downstream-side of the wet chamber or microfluidic device. Therefore, X-ray photons that were scattered from the sample did not pass through the water layer in between the two $\mathrm{Si}_{3} \mathrm{~N}_{4}$ membranes before reaching the detector. The samples were aligned in the focus of the X-ray beam using a calibrated microscope and two dimensional mesh scans were performed on the cells.

For some measurements on hydrated cells, scans with an asymmetric step size, i.e. with a larger step size in vertical direction (slow scan axis) than in the horizontal direction (fast scan axis), were employed. On the one hand this led to a shorter total scan time for covering the same sample area and a lower total average radiation dose per step. On the other hand regions of the cell, which were chemically influenced by radicals that were created in the cell or buffer solution during the measurement at the previous scan line, were skipped since the concentration of radicals, and therefore also the damage in the cell, decreases with larger distance to the exposed positions. This aspect is further discussed in section 6.5.1. 


\subsection{Results from Measurements at the cSAXS Beamline}

In the following section, measurements and results obtained at the cSAXS beamline on fixed-hydrated SK8/18-2 cells with and without a keratin network are presented. An overview of the analyzed measurements along with the employed scan parameters and the estimated average radiation doses per step is shown in Tab6.1.

\begin{tabular}{lllllll}
\hline sample & set-up & $\Delta_{x, y}\left[\mu \mathrm{m}^{2}\right]$ & $N_{x} \times N_{y}$ & $T[\mathrm{~s}]$ & $D[\mathrm{~Gy}]$ \\
\hline BW108, pos. 3A & May 2012 & $1 \times 1$ & $81 \times 81$ & 0.2 & $1.6 \times 10^{5}$ \\
BW108, pos. 3B & May 2012 & $0.2 \times 0.2$ & $71 \times 71$ & 1 & $2.0 \times 10^{7}$ \\
BW108, pos. 3C & May 2012 & $0.1 \times 0.1$ & $81 \times 81$ & 1 & $7.9 \times 10^{7}$ \\
BW108, pos. 4A & May 2012 & $1 \times 1$ & $81 \times 81$ & 0.2 & $1.6 \times 10^{5}$ \\
BW108, pos. 4B & May 2012 & $0.1 \times 0.1$ & $81 \times 81$ & 1 & $7.9 \times 10^{7}$ \\
\hline
\end{tabular}

Tab. 6.1.: Summary of the scan parameters and the estimated average radiation doses per step for the measurements on fixed-hydrated cells at the cSAXS beamline. Here, $\Delta_{x, y}$ are the lateral step sizes, $N_{x}$ and $N_{y}$ the number of scan points in $x$ - and $y$-direction, $T$ is the exposure time and $D$ the estimated average radiation dose per step. All measurements were performed without attenuators in the beam path.

\subsubsection{Images Employing Different X-ray Contrasts}

The measurements on fixed-hydrated samples at the cSAXS beamline were performed without attenuators and without a beamstop and therefore the reconstruction of differential phase contrast (DPC) and transmission images was possible in contrast to the measurements with a beamstop at the ID13 beamline and at the P10 beamline. For the reconstruction of these images as well as the X-ray dark-field image, the Matlab-based analysis script stxm_online.m was used, which was developed at the cSAXS beamline and kindly provided [91, 136.

In Fig. 6.3 and b the visible light phase contrast microscopy image and the fluorescence microscopy image of the keratin network of two SK8/18-2 cells are shown. Fig. 6.3 c-i show an overview of the different contrast mechanisms on the same sample region, i.e. X-ray dark-field contrast, DPC in $x$ - and $y$-direction, and transmission contrast. Additionally, the integrated phase shift from the sample, as determined from the differential phase contrast in $x$ - and $y$-direction, and the differential phase contrast determined from the integrated phase are displayed. The fixed-hydrated 
cells are only visible in the DPC images. The DPC images obtained from the integrated phase are much smoother than the unprocessed DPC images and in particular in the DPCx image, the cell contour and the nucleus can be clearly identified. Furthermore, substructures are visible in the nucleus as well as in the cell body. In particular the dark V-shaped structures in the nucleus in the visible light phase contrast microscopy image, which probably correspond to nucleoli, can be identified
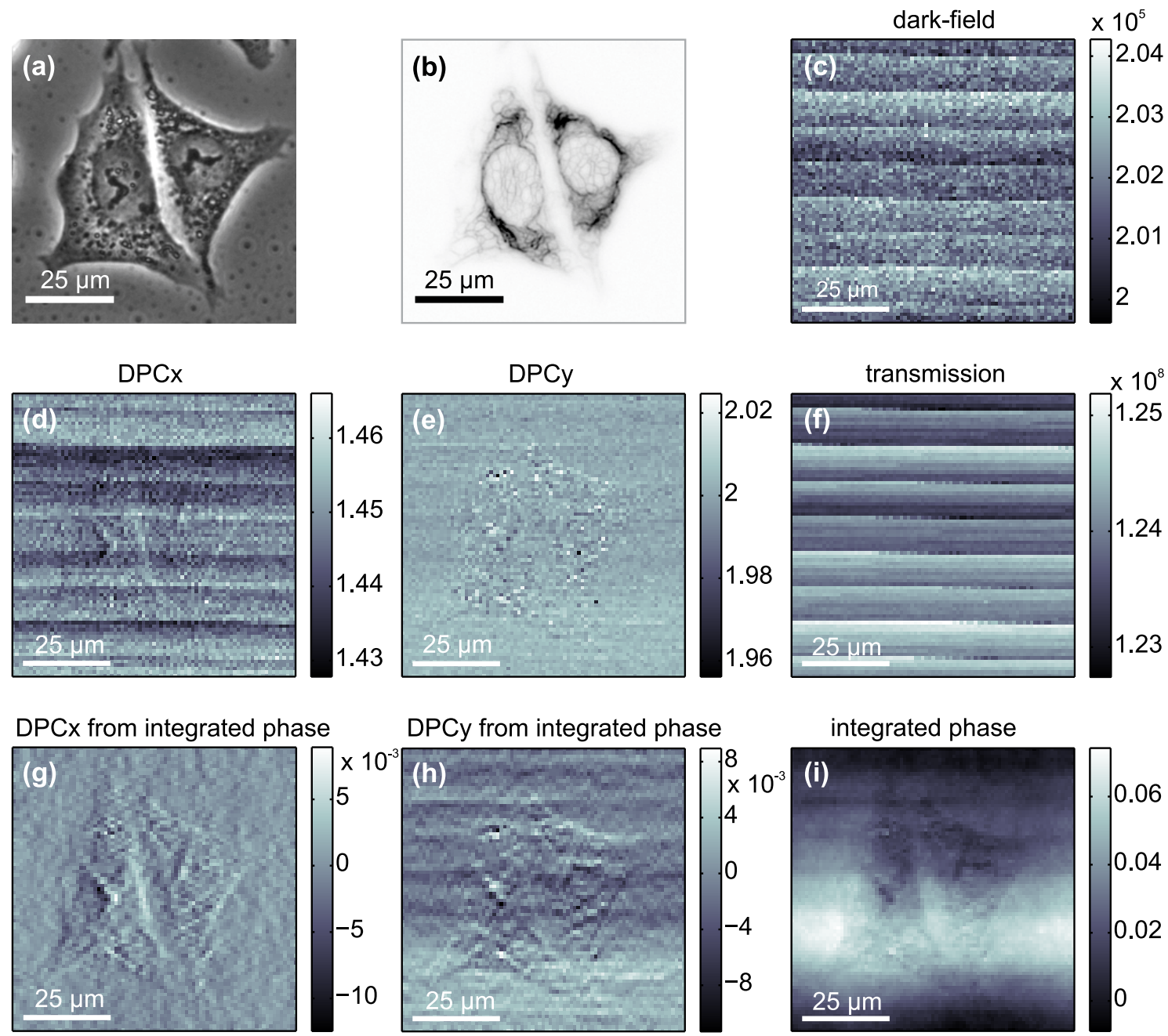

Fig. 6.3.: Microscopy images and different X-ray contrast images of fixed-hydrated SK8/18-2 cells with a keratin network. (a) Visible light phase contrast microscopy image and (b) fluorescence microscopy image of the keratin network in two hydrated SK8/18-2 cells that were fixed with formaldehyde. Images of the same sample region generated by employing different contrast mechanisms: (c) X-ray dark-field contrast, (d) differential phase contrast in $x$-direction, (e) differential phase contrast in $y$-direction, (f) transmission contrast, (g) differential phase contrast in $x$-direction and (h) in $y$-direction obtained by differentiating the integrated phase, (i) integrated phase shift form the sample. (Sample BW108, position 3; cSAXS, May 2012) 

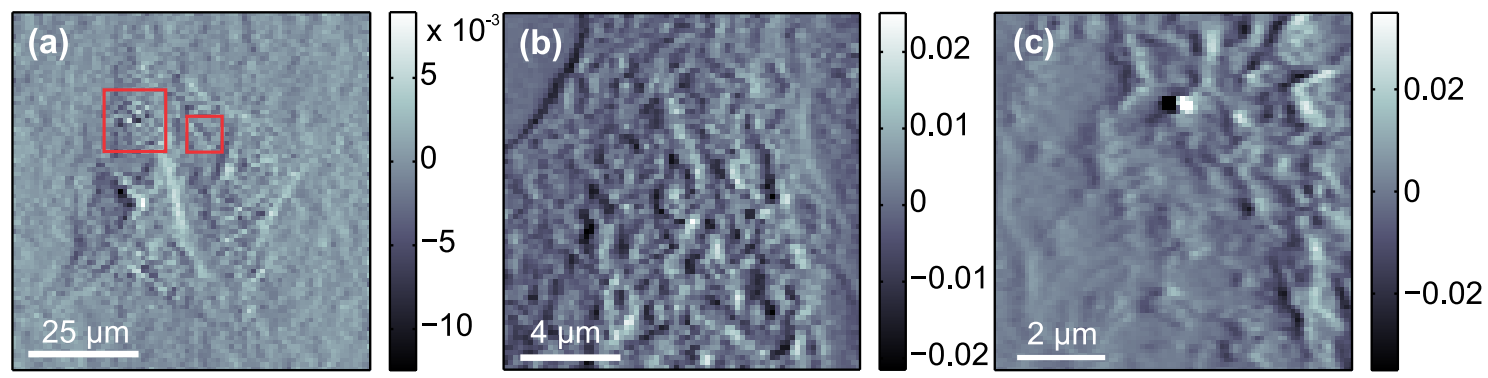

Fig. 6.4.: Region of interest measurements on fixed-hydrated SK8/18-2 cells with a keratin network. (a) Selection of two different regions-of-interest for measurements with smaller step sizes marked by red squares. (b) Region of interest measurement on the left region in panel (a) with a step size of $200 \mathrm{~nm}$. (c) Region of interest measurement on the right region in panel (a) with a step size of $100 \mathrm{~nm}$. All three images show the differential phase contrast in $x$-direction obtained by differentiating the integrated phase. (Sample BW108, position 3; cSAXS, May 2012)

in the DPC images obtained from the integrated phase as well. The X-ray darkfield and transmission image shown no contrast between the cellular material and the surrounding buffer solution for all measured fixed-hydrated cells. Horizontal stripes in the images with very low contrast can be attributed to small changes in the electron beam current, which are related the operation mode (top-up mode) of the storage ring.

Finer scans with smaller step sizes and longer exposure times were performed on two different regions, which are indicated by red squares in Fig. 6.4 a. The DPCx image obtained from the integrated phase for the region of interest scans are presented in Fig. 6.4b and c. In both scans, rough structures with structural sizes in the order of $300 \mathrm{~nm}$ can be identified. This observation is in line with the visible light phase contrast image in Fig. 6.3 , which shows roundish bright and dark structures close to the nucleus that probably correspond to cell organelles like the endoplasmic reticulum, the Golgi apparatus or lysozymes. The fluorescence microscopy image of the keratin network in Fig. 6.3p displays several thick keratin bundles in the same regions. However, the network morphology cannot be directly correlated with structures in the DPCx images.

Similar measurements were performed on a keratin-free cell to compare the results to the keratin-containing cell presented above. Fig. 6.5 and b show the visible light phase contrast and the fluorescence microscopy images, which validate that the cell does not contain keratin. The DPCx image recorded on the same sample region is displayed in Fig. 6.5c. Here, the cell contour can be only identified in the top-left and top-right region of the cell. The other edges of the cell seem to be too flat to be visible in the DPCx image. Furthermore, the nucleus and substructures in the 

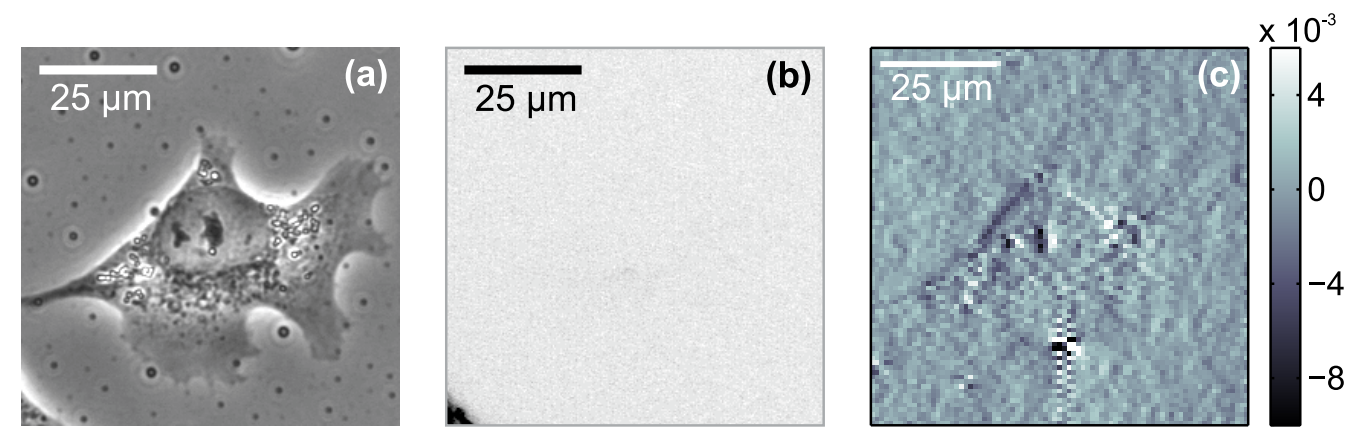

Fig. 6.5.: Microscopy images and DPCx image of a fixed-hydrated SK8/18-2 cell without a keratin network. (a) Visible light phase contrast and (b) fluorescence microscopy images of a hydrated keratin-free cell that was fixed with formaldehyde. (c) Image of the same sample region showing the differential phase contrast in $x$-direction obtained by differentiating the integrated phase. (Sample BW108, position 4A; cSAXS, May 2012)

nucleus can be identified. The spot in the center of the bottom half of the DPCx image probably corresponds to a dirt particle on the $\mathrm{Si}_{3} \mathrm{~N}_{4}$ membrane.

A finer measurement with a smaller step size and a longer exposure time was performed on the marked region in Fig. 6.6 and the DPCx image from this scan is shown in Fig. 6.6b. For the keratin-free cell, a substructure is visible in the region of interest scan, which seems to be similar to the substructures observed for the cell with a keratin network displayed in Fig 6.4 ent structures in the DPCx images do not correspond to the keratin network, but to organelles or other cytoskeletal proteins that are present in the keratin-containing cells as well as in the keratin-free cells. Therefore, the signal from the keratin network does not exceed the contributions from the other cellular material or dominate
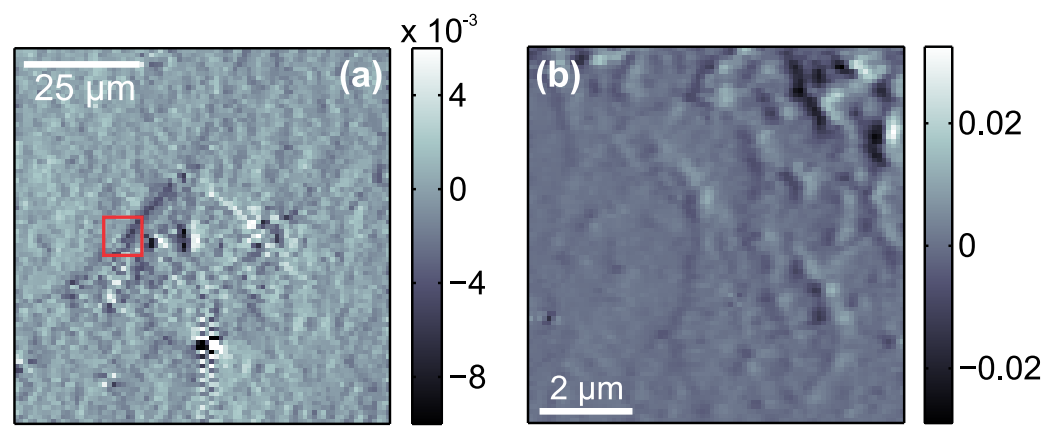

Fig. 6.6.: Region of interest measurement on a fixed-hydrated SK8/18-2 cell without a keratin network. (a) Selection of a region of interest, marked by a red box, for a measurement with a smaller step size. (b) Region of interest scan with a step size of $100 \mathrm{~nm}$. Both images show the differential phase contrast in $x$-direction obtained by differentiating the integrated phase. (Sample BW108, position 4; cSAXS, May 2012) 
the total signal, which is in line with the observation for the X-ray dark-field images of freeze-dried samples as presented and discussed in chapter 5 . However, the possibility of label-free X-ray imaging of fixed-hydrated cells with a resolution of about $100 \mathrm{~nm}$, as determined by the step size and the beam dimension, could be demonstrated.

\subsubsection{Radial Intensity of Averaged Scattering Patterns}

For further analysis of the measurements on hydrated cells, the scattering patterns in three different regions of the scan on the cells with a keratin network were averaged. Fig. 6.7 $\mathrm{A}$ shows the regions that were used for averaging, i.e. the nucleus, the cell body and an empty region. The corresponding averaged scattering patterns

(a)
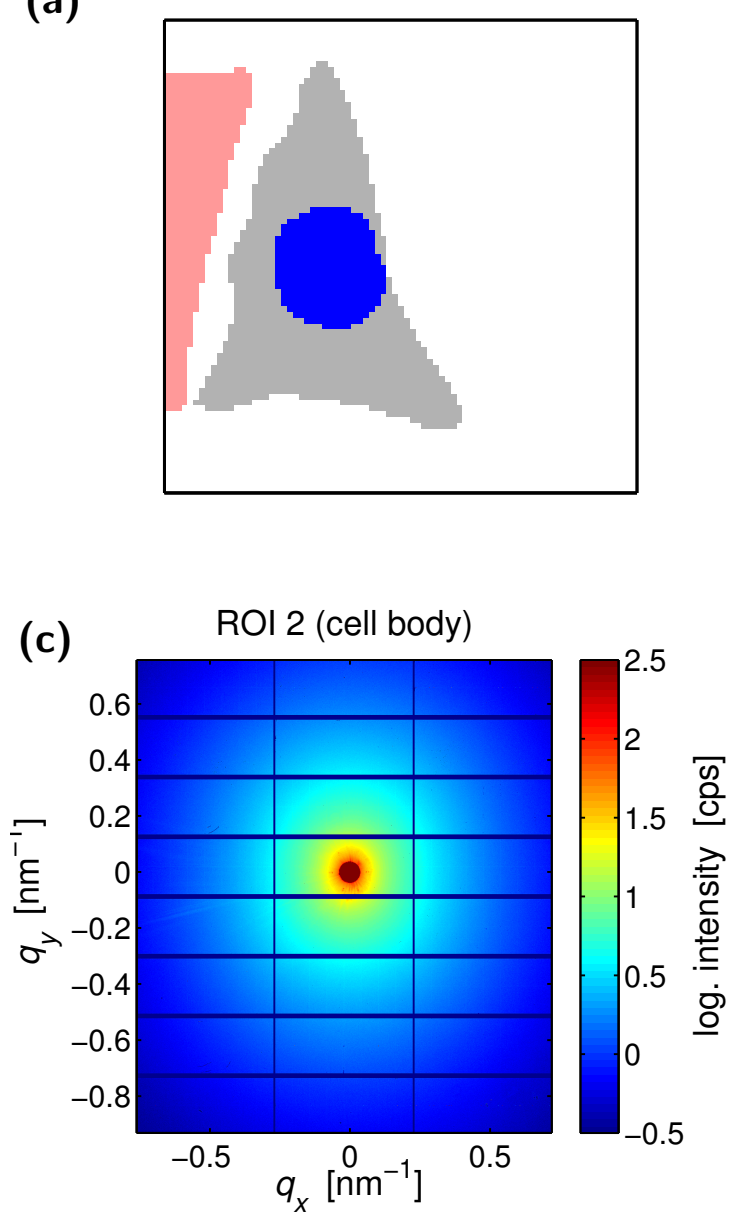

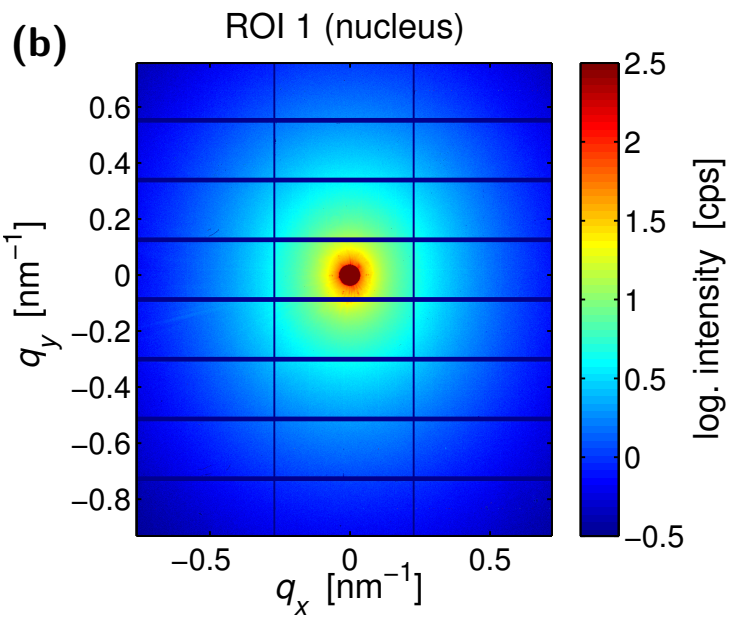

(d)

ROI 3 (empty)

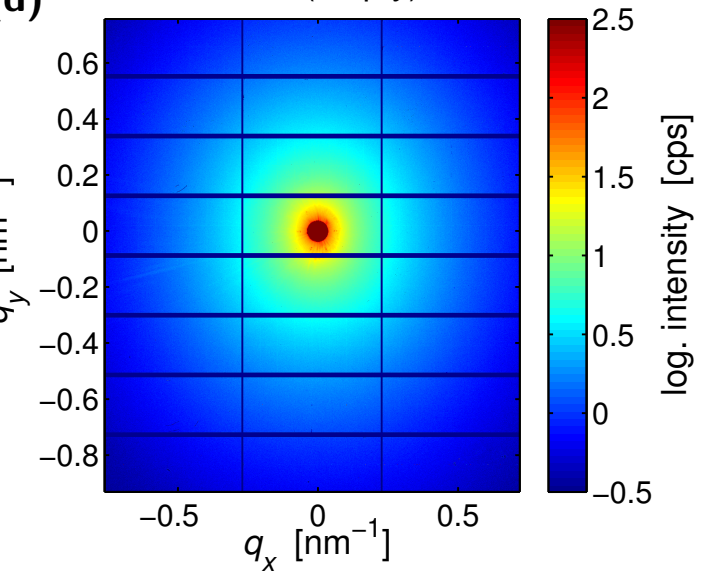

Fig. 6.7.: Averaged scattering patterns from three different scan regions. (a) Selection of three different scan regions, i.e. the nucleus (blue, ROI 1), the cell body (gray, ROI 2) and an empty region (red, ROI 3). (b) Averaged scattering pattern from the nucleus, (b) the cell body and (c) the empty region. (Sample BW108, position 3A; cSAXS, May 2012) 


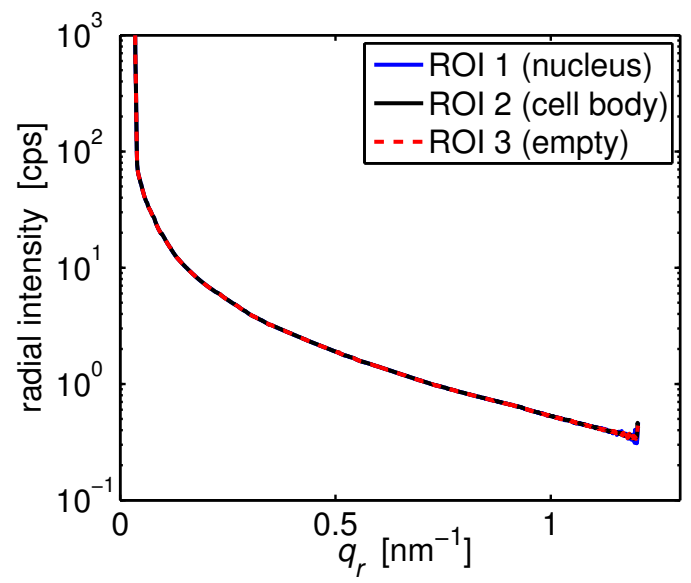

Fig. 6.8.: Radial intensity profiles from averaged scattering patterns from the nuclear region, the cell body and the empty region. (Sample BW108, position 3A; cSAXS, May 2012)

are displayed in Fig. 6.7b-d. The scattering patterns show no difference between scattering from the nucleus, the cell body and the empty region. The hexagonally arranged scattering maxima centered around the primary beam in a distance of about $0.06 \mathrm{~nm}^{-1}$, can be attributed to scattering from the other zone plates that are located on the same chip as the employed zone plate. Further, streaks in the scattering patterns centered around the primary beam are caused by scattering at the edges of the order sorting aperture.

The averaged scattering patterns were integrated in azimuthal direction yielding the radial intensity profiles presented in Fig. 6.8. The three curves coincide, meaning that no scattering signal form the cells could be detected above the background scattering using this set-up. This observation agrees with the previous finding that the cells could not be identified in the X-ray dark-field image (compare Fig. 6.3.). Therefore, the set-up needs to be further improved in terms of the background scattering, before being able to record the scattering signal from hydrated cellular samples. 


\subsection{Results from Measurements at the ID13 Beamline}

In the following section, an example for results from measurements on fixed-hydrated SK8/18-2 cells at the ID13 beamline is presented. Best results were obtained for two measurements with an asymmetric step size, i.e. with a larger step size in vertical than in horizontal direction (compare section 6.5). Due to the severe radiation damage to the fixed-hydrated samples at the ID13 beamline, it was not possible to perform finer region of interest scans on these samples as presented above for the measurements at the cSAXS beamline.

Results from one measurement on a fixed-hydrated SK8/18-2 cell, are presented in this section and the results from the second measurement on a different cell are displayed in the supplementary material in section C.1. An overview of the measurements presented here on fixed-hydrated cells taken at the ID13 beamline along with the employed scan parameters and the estimated average radiation doses per step is shown in Tab.6.2.

\begin{tabular}{|c|c|c|c|c|c|}
\hline sample & set-up & $\Delta_{y, z}\left[\mu \mathrm{m}^{2}\right]$ & $N_{y} \times N_{z}$ & $T[\mathrm{~s}]$ & $D[\mathrm{~Gy}]$ \\
\hline BW171, pos. 1 & Nov. 2012 & $0.3 \times 1$ & $101 \times 144$ & 0.5 & $5.5 \times 10^{6}$ \\
\hline BW177, pos. 2 & Nov. 2012 & $0.3 \times 1$ & $121 \times 100$ & 0.5 & $5.5 \times 10^{6}$ \\
\hline
\end{tabular}

Tab. 6.2.: Summary of the scan parameters and the estimated average radiation doses per step for the measurements on fixed-hydrated cells at the ID13 beamline. Here, $\Delta_{y, z}$ are the lateral step sizes, $N_{y}$ and $N_{z}$ the number of scan points in $y$ - and $z$-direction, $T$ is the exposure time and $D$ the estimated average radiation dose per step. All measurements were performed without attenuators in the beam path.

\subsubsection{X-Ray Dark-Field Images}

To increase the contrast in the X-ray dark-field images of hydrated samples measured at the ID13 beamline, only the scattered intensity in a ring-like region around the beam center on the detector was integrated. Best results were obtained with an inner radius of $q_{r, i}=40$ pixel $=0.175 \mathrm{~nm}^{-1}$ and an outer radius of $q_{r, o}=60$ pixel $=0.262 \mathrm{~nm}^{-1}$. The detector mask that was used for the calculation of the X-ray dark-field images is shown in Fig. 6.9 and the valid pixels are depicted in gray. 


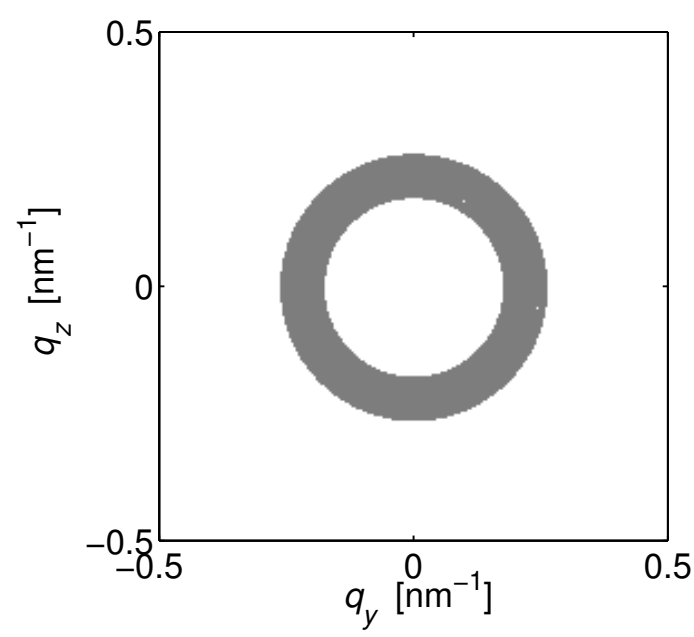

Fig. 6.9.: Detector mask used for the calculation of the X-ray dark-field images of hydrated samples measured at the ID13 beamline. The valid pixels are displayed in gray.

Fig. 6.10a and b show the visible light phase contrast and the fluorescence microscopy images of a fixed-hydrated SK8/18-2 cell. The fluorescence microscopy image shows only a blurred fluorescence signal and no keratin network. The X-ray dark-field image taken on the same sample area is displayed in Fig. 6.10k. The cell contour can be identified in the X-ray dark-field image and the nucleus can be distinguished from the cell body. Both for the cell body and the nucleus, the signal is not homogeneous over the whole area. Dark spots in the nucleus that are visible in the visible light phase contrast image can be identified in the X-ray dark-field image as well. However, the contrast between the cellular material and the empty substrate is quite low. Additionally, the scattering signal from some regions of the cell is not higher than the signal from the empty regions. Similar results were obtained for the second measurement, which is displayed in the appendix in Fig. C.1. For a higher primary beam intensity, a better contrast in the

(a)

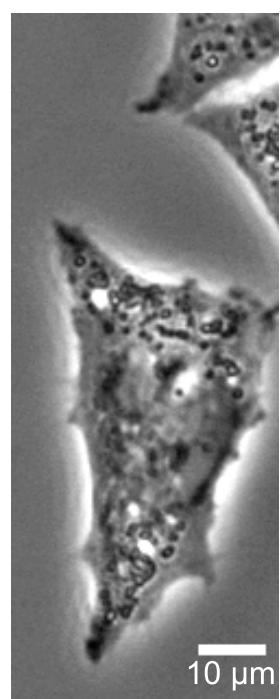

(b)

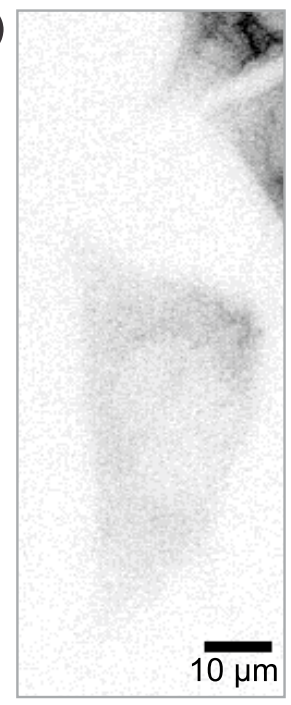

(c)

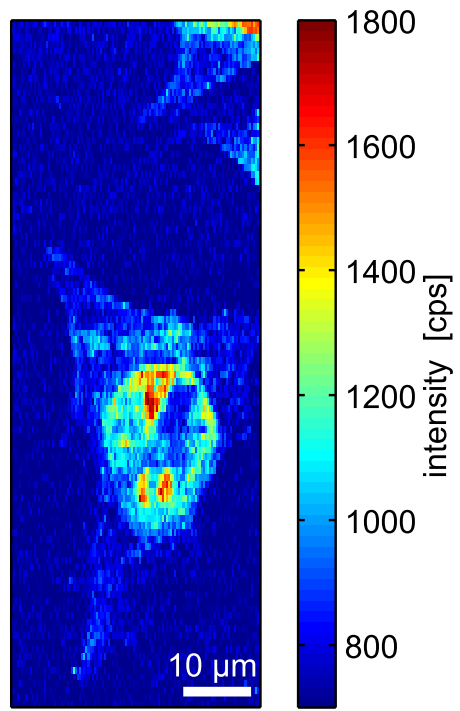

Fig. 6.10.: Microscopy images and X-ray dark-field image of a fixed-hydrated SK8/18-2 cell. (a) Visible light phase contrast and (b) fluorescence microscopy images of a hydrated SK8/18-2 cells that was fixed with formaldehyde. (c) X-ray dark-field image recorded on the same sample region. (Sample BW177, position 2; ID13, Nov. 2012) 
X-ray dark-field image could probably be obtained.

\subsubsection{Radial Intensity of Averaged Scattering Patterns}

Scattering patterns from three different regions of the scan, i.e. the nucleus, the cell body and an empty region, as marked in Fig. 6.12a, were averaged. Here, the background region was chosen roughly in the same scan lines where the cell was located, which ensured similar measurement conditions for all three regions and accounts for the decreasing primary beam intensity during the scan due to the decreasing ring current. This is particularly important for weakly scattering samples, because otherwise the background signal might be higher or lower than the cell signal at high

(a)

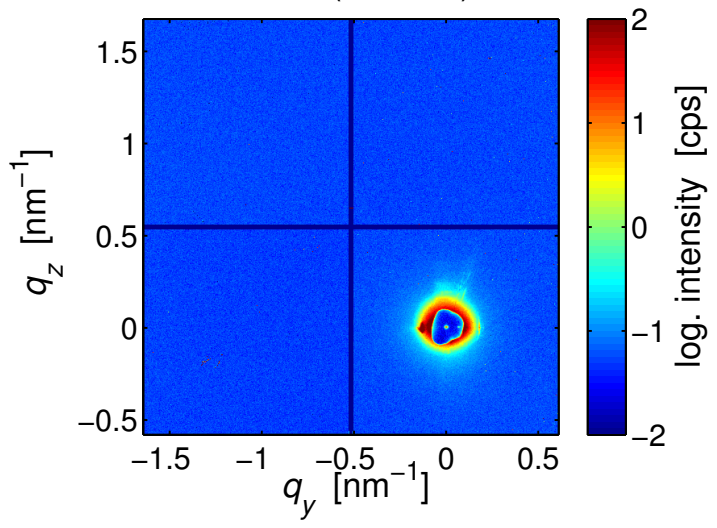

(c)

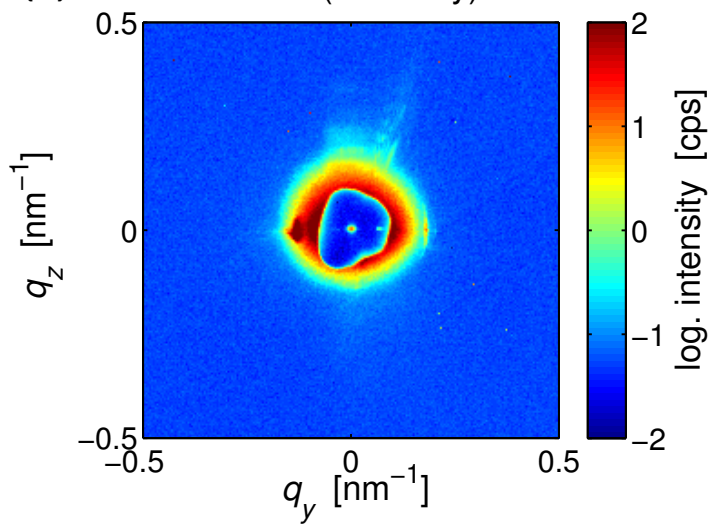

(b)

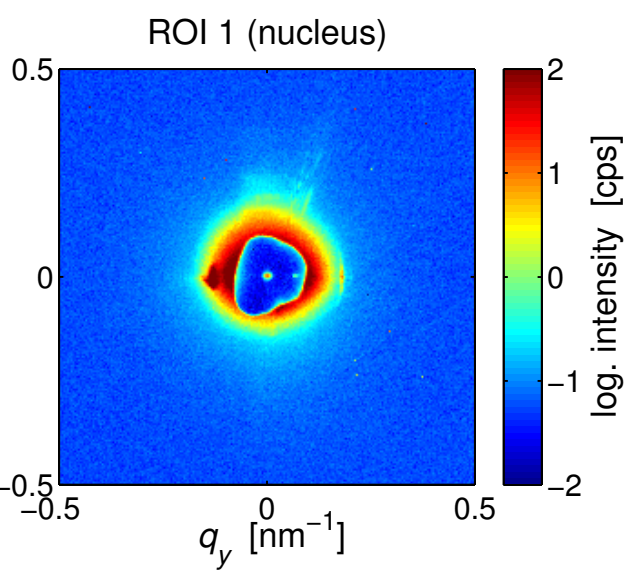

(d)

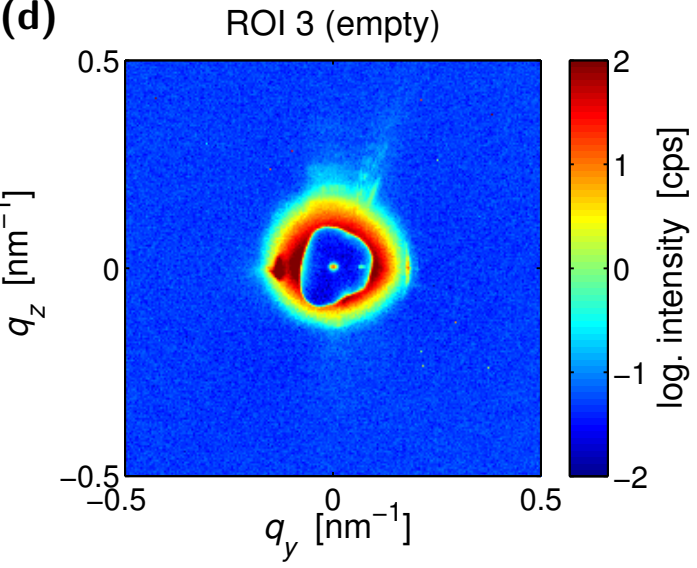

Fig. 6.11.: Averaged scattering pattern from three different scan regions on a fixedhydrated SK8/18-2 cell. (a) Complete averaged scattering pattern from the nuclear region. (b) Magnification of the $q$-region around the beam center of the averaged scattering pattern from the nuclear region, (c) the cell body and (d) the empty region. (Sample BW177, position 2; ID13, Nov. 2012) 
(a)

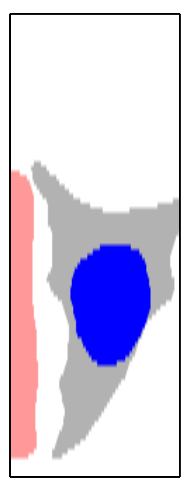

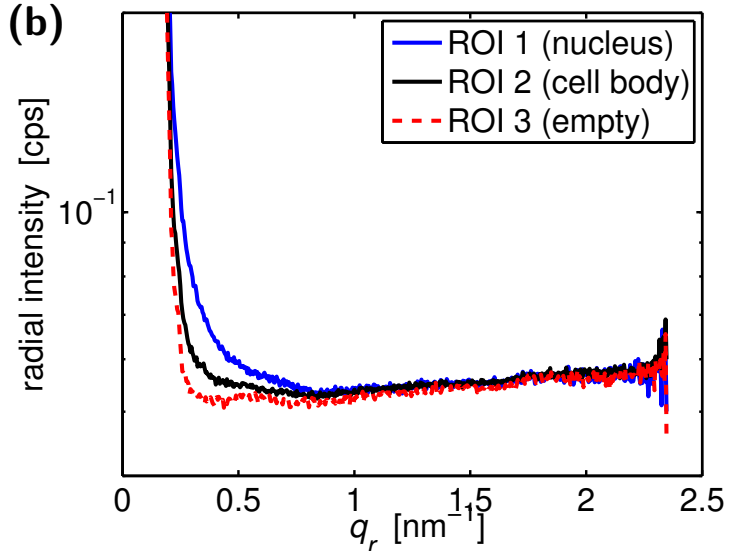

Fig. 6.12.: Radial intensity profiles of averaged scattering patterns. (a) Selection of three different scan regions, i.e. the nucleus (blue, ROI 1), the cell body (gray, ROI 2) and an empty region (red, ROI 3). (b) Radial intensity profiles of averaged scattering patterns from the nuclear region, the cell body and the empty region. (Sample BW177, position 2; ID13, Nov. 2012)

$q_{r}$-value, which induces artifacts upon background subtraction. The averaged scattering patterns are displayed in Fig. 6.11. Fig. 6.11 a shows the complete averaged scattering pattern from the nuclear region. For the averaged scattering patterns in Fig. 6.11b-d, a smaller $q$-range around the beam center is displayed for all three regions. Only a small difference between the averaged scattering pattern from the nucleus and the empty region is visible in the low $q$-region close to the beamstop. The streaks in the upper part of the scattering patterns are due to the aperture, which was used for cleaning of the beam profile.

The averaged scattering patterns were integrated in azimuthal direction yielding the radial intensity profiles presented in Fig. $6.12 \mathrm{~b}$. The noise in the radial intensity profiles is much stronger as compared to similar measurement on freeze-dried samples at the ID13 beamline. However, the radial intensity profiles from the nucleus and the cell body are well above the profile from the background region. Furthermore, the scattering signal from the nucleus is stronger than the signal form the cell body, as would be expected since the nucleus is thicker than the cell body. The high packing density of the chromatin in the nucleus might also lead to a stronger scattering signal as compared to the cell body.

The intensity decays of the background corrected average radial intensity profiles were fitted with power law functions. Fig. 6.13 shows the resulting fits along with the background corrected radial intensity profiles from the nucleus and the cell body. Despite the noise in the radial intensity profiles, the averaged scattering signal reaches up to about $1 \mathrm{~nm}^{-1}$. The fits describe the data very well, except for the 
first data points in the low $q_{r}$-region, which were left out for the fits. The power law exponents are in range from -2.5 to -3 for both region and in particular, the power law exponents obtained for the fixed-hydrated cell are higher than the ones obtained for the freeze-dried cells at the same set-up. However, the absolute value of the power law exponents for the fixed-hydrated cells might be affected by the strong noise in the data.

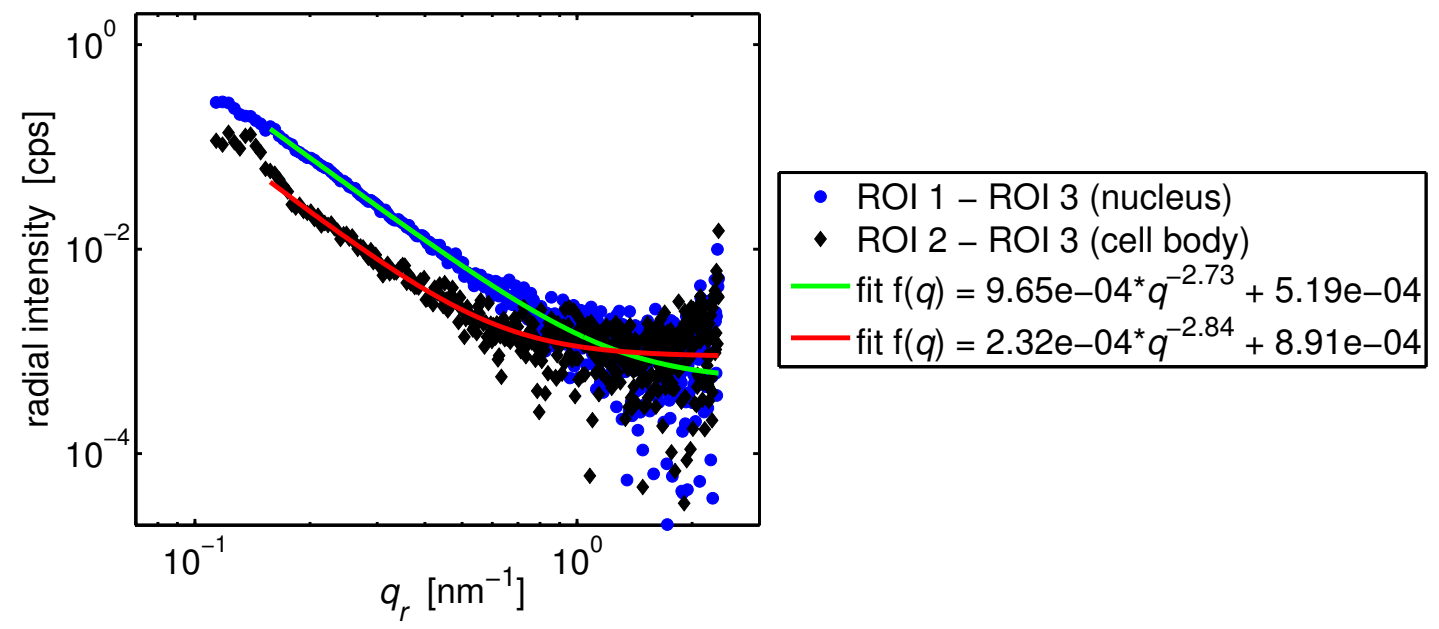

Fig. 6.13.: Fitting of two power laws to the background corrected average radial intensity profiles of the nucleus and the cell body, respectively. The $q_{r}$-ranges over which the fit functions are plotted correspond to the regions that were used for fitting. (Sample BW177, position 2; ID13, Nov. 2012) 


\subsection{Results from Measurements at the P10 Beamline}

In the following section, an example for measurements and results from fixedhydrated SK8/18-2 cells obtained at the P10 beamline is presented. X-ray dark-field images as well as the radial intensity profiles and the corresponding fits with power laws from the other measurements are documented in the supplementary material in section C.2. The power law exponents obtained from fits to the radial intensity of averaged scattering pattern for all fixed-hydrated cells measured at the P10 beamline are compared. An overview of the analyzed measurements on fixed-hydrated SK8/18-2 cells taken at the P10 beamline along with the employed scan parameters and the estimated average radiation doses per step is shown in Tab. 6.3.

\begin{tabular}{lllllll}
\hline sample & set-up & $\Delta_{y, z}\left[\mu \mathrm{m}^{2}\right]$ & $N_{y} \times N_{z}$ & $T[\mathrm{~s}]$ & $T_{\text {att }}$ & $D[\mathrm{~Gy}]$ \\
\hline BW139, pos. 3 & Oct. 2012 & $0.5 \times 0.5$ & $201 \times 281$ & 0.05 & 1 & $3.9 \times 10^{7}$ \\
BW143, pos. 3 & Oct. 2012 & $0.5 \times 0.4$ & $161 \times 251$ & 0.05 & 1 & $4.9 \times 10^{7}$ \\
BW145, pos. 2 & Oct. 2012 & $0.5 \times 0.5$ & $241 \times 241$ & 0.06 & 0.263 & $1.2 \times 10^{7}$ \\
BW145, pos. 3 & Oct. 2012 & $0.5 \times 0.5$ & $241 \times 201$ & 0.08 & 0.263 & $1.6 \times 10^{7}$ \\
BW145, pos. 4 & Oct. 2012 & $0.5 \times 0.5$ & $241 \times 241$ & 0.08 & 0.263 & $1.6 \times 10^{7}$ \\
BW145, pos. 5 & Oct. 2012 & $0.5 \times 0.5$ & $241 \times 241$ & 0.09 & 0.263 & $1.9 \times 10^{7}$ \\
BW156, pos. 3 & Oct. 2012 & $0.5 \times 0.5$ & $201 \times 201$ & 0.05 & 1 & $3.9 \times 10^{7}$ \\
BW235, pos. 1 & March 2013 & $0.5 \times 0.5$ & $161 \times 161$ & 0.05 & 1 & $2.9 \times 10^{7}$ \\
BW237, pos. 1 & March 2013 & $1 \times 1$ & $101 \times 101$ & 0.05 & 1 & $7.2 \times 10^{6}$ \\
BW237, pos. 2 & March 2013 & $1 \times 1$ & $101 \times 101$ & 0.05 & 1 & $7.2 \times 10^{6}$ \\
BW237, pos. 3 & March 2013 & $1 \times 1$ & $101 \times 101$ & 0.05 & 1 & $7.2 \times 10^{6}$ \\
\hline
\end{tabular}

Tab. 6.3.: Summary of the scan parameters and the estimated average radiation doses per step for the measurements on fixed-hydrated cells at the P10 beamline. Here, $\Delta_{y, z}$ are the lateral step sizes, $N_{y}$ and $N_{z}$ the number of scan points in $y$ - and $z$-direction, $T$ is the exposure time, $T_{\text {att }}$ the attenuator transmission and $D$ the estimated average radiation dose per step.

\subsubsection{X-Ray Dark-Field Images}

To increase the contrast in X-ray dark-field images of hydrated samples measured at the $\mathrm{P} 10$ beamline, only the scattered intensity in a ring-like region around the beam center on the detector was integrated. Best results were obtained with an inner radius of $q_{r, i}=80$ pixel $=0.105 \mathrm{~nm}^{-1}$ and an outer radius of $q_{r, o}=150$ pixel $=$ 
$0.198 \mathrm{~nm}^{-1}$. The detector mask that was used for the calculation of the X-ray darkfield images is shown in Fig. 6.14 with the valid pixels depicted in gray. Here, additionally the pseudo-pixels between the modules of the detector, the beamstop, and the strong streaks from the KB beam and scattering from the apertures are masked. The detailed masks for the streaks and the beamstop might vary for different samples, depending on whether the set-up or the alignment was changed between the measurements.

An example for an X-ray dark-field image of two fixed-hydrated SK8/18-2 cells is displayed in Fig. 6.15c and the corresponding visible light phase contrast microscopy image and the fluorescence microscopy image of the keratin network are shown in Fig. $6.15 \mathrm{a}$ and $\mathrm{b}$. The cells can be clearly identified in the X-ray dark-field image and the nuclei can be distinguished from the cell

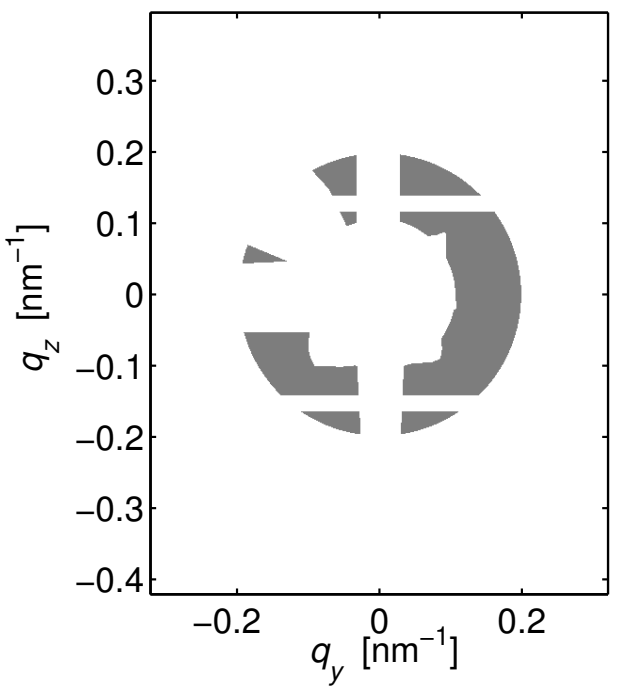

Fig. 6.14.: Detector mask used for calculation of X-ray dark-field images of hydrated samples measured at the P10 beamline. The valid pixels are displayed in gray. bodies due to a much stronger scattering signal. Both in the nuclei and in the cell bodies, substructures are visible. In the X-ray dark-field image, substructures in the cell body are observed particularly in close vicinity to the nucleus. In the same regions, substructures can be identified in the visible light phase contrast image, which
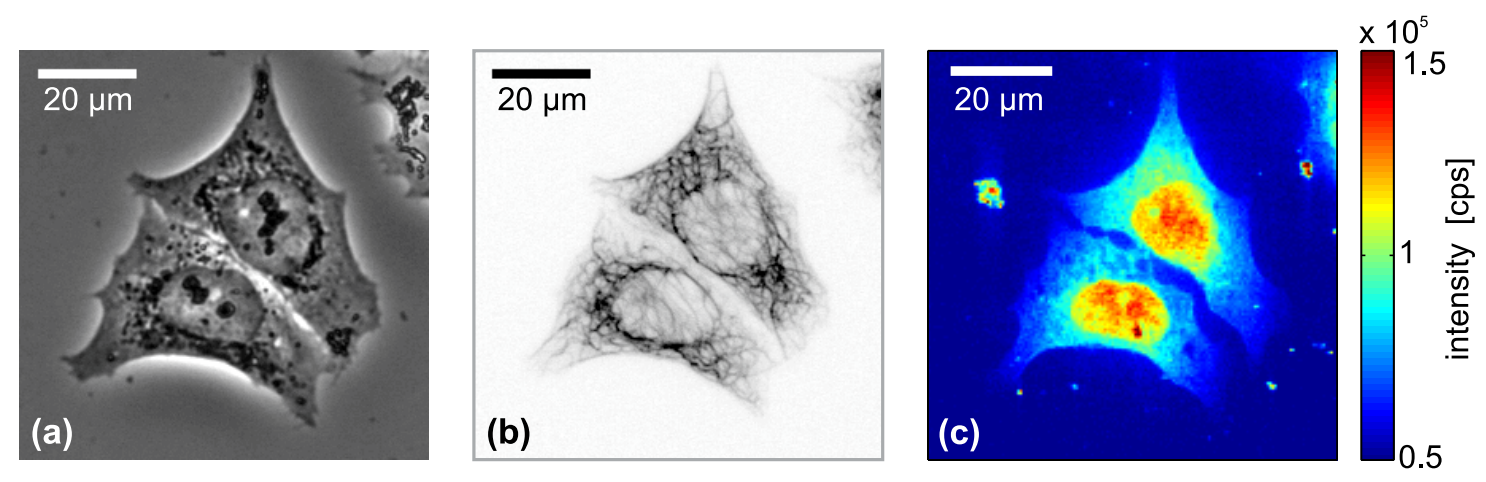

Fig. 6.15.: Microscopy images and X-ray dark-field image of a fixed-hydrated SK8/18-2 cell. (a) Visible light phase contrast microscopy image and (b) fluorescence microscopy image of the keratin network in hydrated SK8/18-2 cells that was fixed with formaldehyde. (c) X-ray dark-field image of the same sample region as in panel (a) and (b). (Sample BW235, position 1; P10, March 2013) 
probably correspond to cell organelles or large vesicles. The scattering signal is also stronger in regions where the keratin network is dense, i.e. close to the nucleus, but pronounced bundles that are visible in the fluorescence microscopy image cannot be identified in the X-ray dark-field image.

\subsubsection{Radial Intensity of Averaged Scattering Patterns}

Three different scan regions, i.e. the nucleus, the cell body and an empty region, were selected as shown in Fig. 6.16a. For the measurements on hydrated cells it turned out to be important to choose the background region roughly from the same scan lines where the cell was located. Otherwise at high $q_{r}$-values the background

(a)

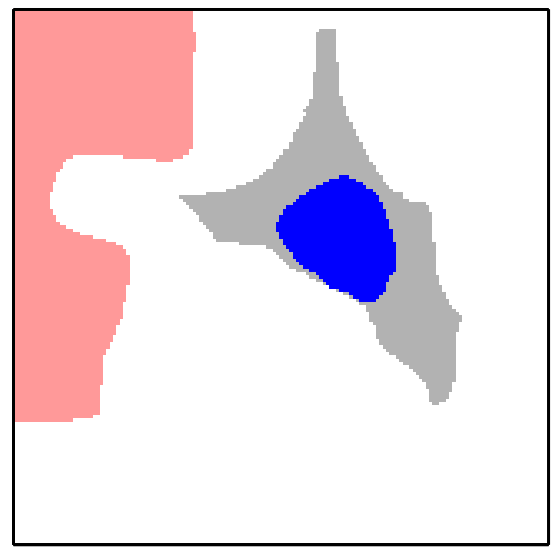

(c)

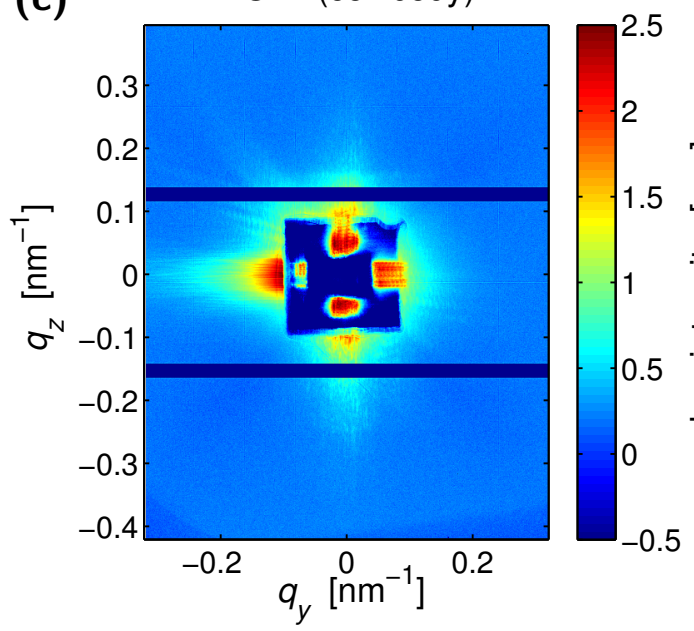

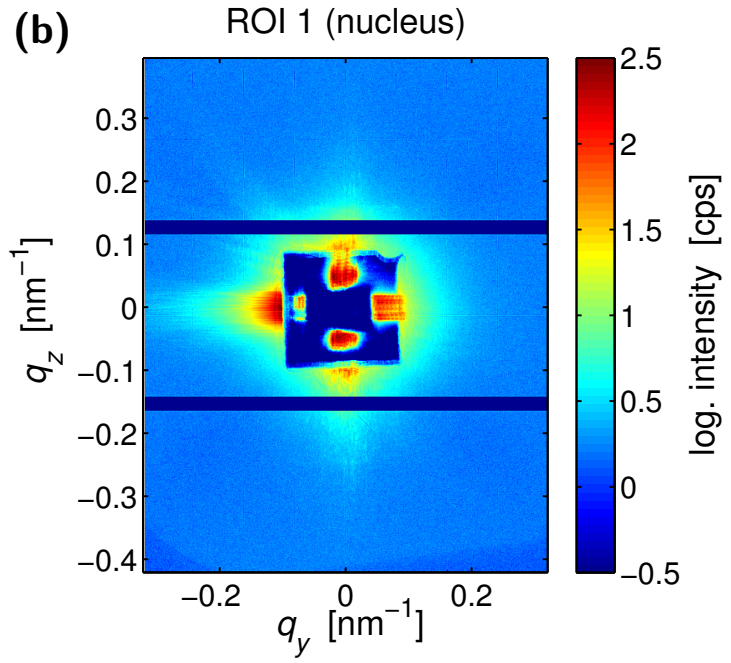

(d)

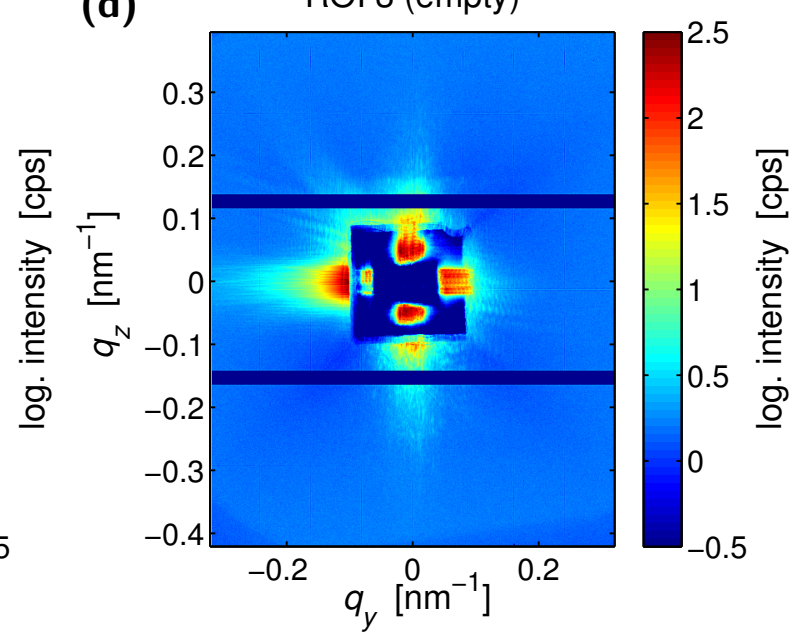

Fig. 6.16.: Averaged scattering patterns from three different scan regions. (a) Selection of three different scan regions, i.e. the nucleus (blue, ROI 1), the cell body (gray, ROI 2) and an empty region (red, ROI 3). (b) Averaged scattering pattern from the nucleus, (b) the cell body and (c) the empty region. (Sample BW235, position 1; P10, March 2013) 
signal might be higher or lower than the averaged signal from the cell, which led to problems during background subtraction. These changes in background scattering during the scan can be explained by tiny changes in the set-up, e.g. due to drifts of the soft-edge apertures, which also lead to changes in the primary beam intensity as well as changes in the background scattering like the intensity of the streaks from the KB beam or the apertures. This problem becomes apparent for hydrated cells due to the much weaker scattering signal compared to the measurements on freezedried cells.

All scattering patterns in the selected regions were averaged, yielding the averaged scattering patterns shown in Fig. 6.16b-d. The scaling of the intensity is the same for all three images. The primary beam in the center of the images is blocked by a rectangular beamstop to prevent detector saturation. In the empty image strong background scattering from the KB optics and the soft-edge apertures in form of intense streaks is visible. In the averaged scattering patterns from the cell body and the nucleus, the scattering signal from the cell can be observed. As expected from the X-ray dark-field image, the scattering signal from the nucleus is stronger than the signal from the cell body, which can be explained by the higher thickness of the nucleus and the more densely packed chromatin in the nucleus as compared the structures in the cell body. In the lower part of the scattering patterns, the scattered intensity is blocked by the flight tube.

Azimuthal integration of the averaged scattering patterns in one angular segment yields the three radial intensity profiles presented in Fig. 6.17a and subtraction of the background profile from the cell profiles results in the curves displayed in Fig.

(a)

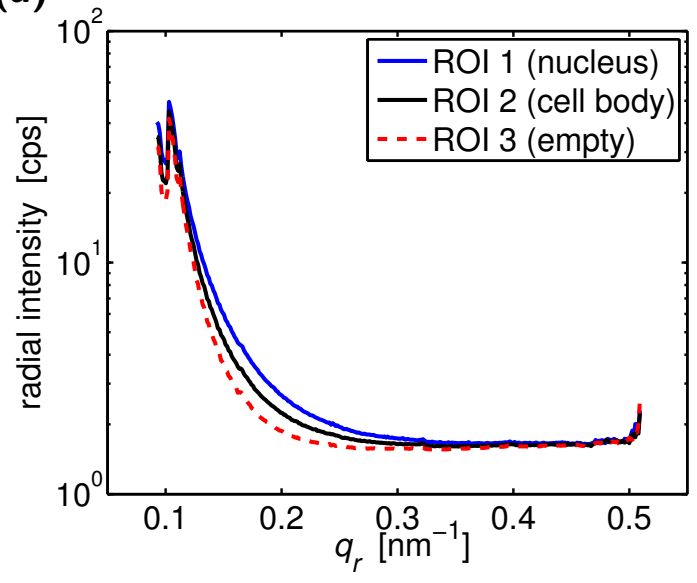

(b)

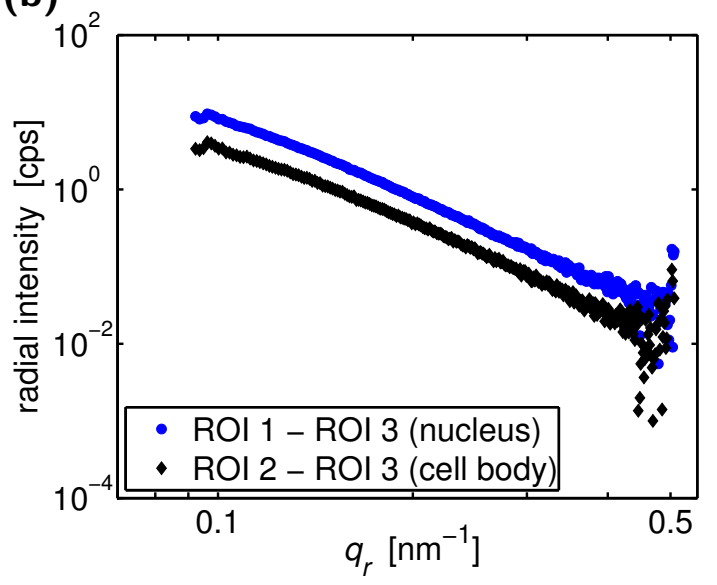

Fig. 6.17.: Radial intensity profiles of averaged scattering patterns. (a) Radial intensity profiles from the nuclear region, the cell body and the empty region. (b) Background corrected radial intensity profiles from the nuclear region and the cell body. (Sample BW235, position 1; P10, March 2013) 


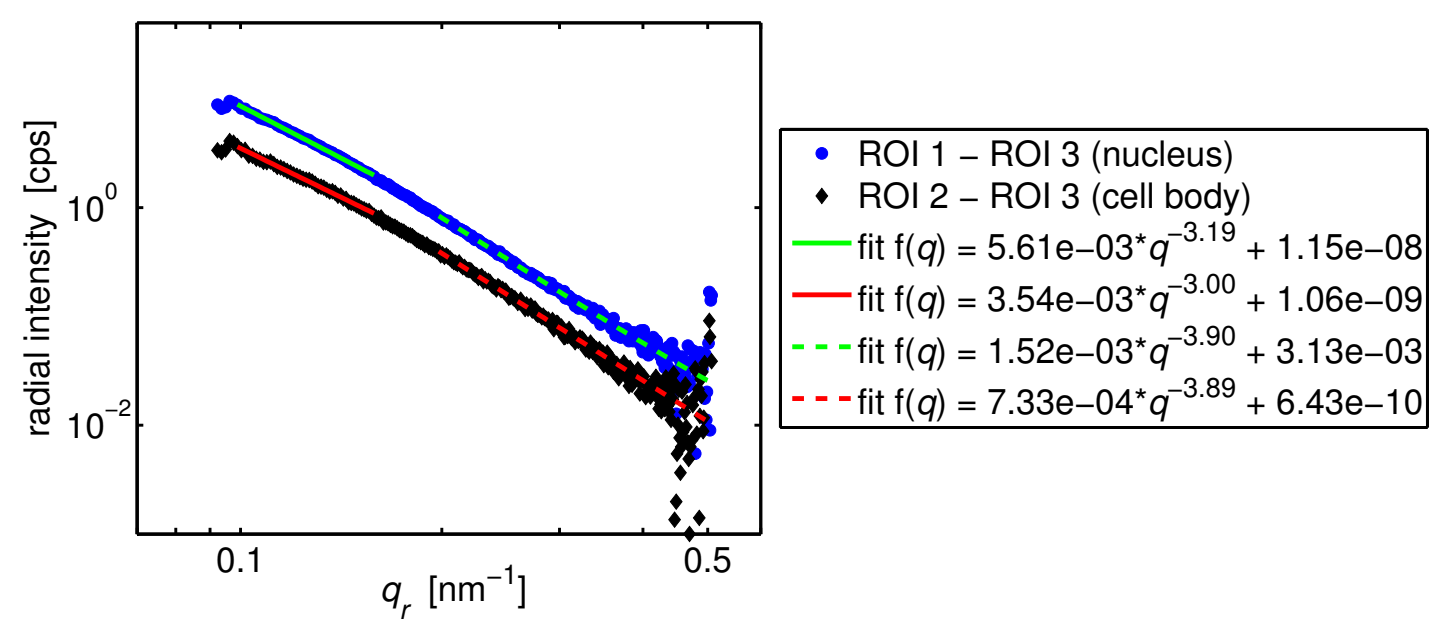

Fig. 6.18.: Fitting of two power laws to the low $q_{r}$-region and high $q_{r}$-region of the background corrected average radial intensity profiles from the nucleus and the cells body, respectively. The $q_{r}$-ranges over which the fit functions are plotted correspond to the regions that were used for fitting. (Sample BW235, position 1; P10, March 2013)

6.17b. The background corrected profiles show a smooth intensity decay, which is cut off at high $q_{r}$-values by the detector before reaching the noise level. The slight increase in noise for the data points at highest $q_{r}$-values is on the one hand related to the low number of photon counts for these pixels and on the other hand to the lower number of pixel that are available for azimuthal integration. Both intensity profiles have a concave shape.

To quantify the intensity decay, two power laws were fitted to the background corrected radial intensity profiles from the nucleus and the cell body in the low $q_{r^{-}}$and high $q_{r}$-region, respectively. Fig. 6.18 shows the fits along with the measured data and the $q_{r}$-ranges over which the fit functions are plotted correspond to the regions that were used for fitting. The data in the respective regions are well described by the power laws. Comparing the fit results obtained for the nucleus and the cell body, the exponent in the two different $q_{r}$-regions are very similar.

\subsubsection{Comparison of Power Law Exponents}

The same analysis scheme as presented above was applied to several fixed-hydrated cells that were measured at the P10 beamline in October 2012 and March 2013. The $\mathrm{X}$-ray dark-field images, the visible light microscopy images as well as the radial intensity profiles along with the power law fits and maps of the selected scan regions for averaging of the scattering patterns are documented in the supplementary information in section C.2 for all analyzed fixed-hydrated cells at the P10 beamline. The measurements on fixed-hydrated cells can be grouped into measurements taken 


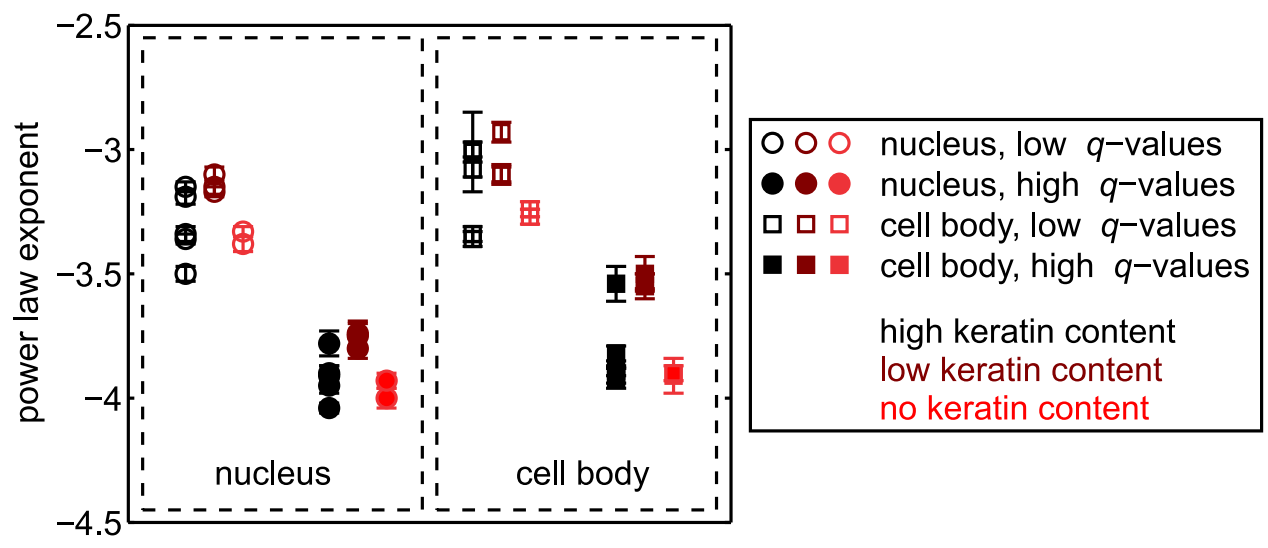

Fig. 6.19.: Comparison of the power law exponents for fixed-hydrated cells. For each group of data points the first column corresponds to cells with a keratin network, the second column corresponds to cells with little keratin content and the third column corresponds to cells without keratin. The error bars indicate the errors obtained from the fits with a power law. All measurements shown in this figure were performed without attenuators. (P10, Oct. 2012 and March 2013)

without attenuators and measurements taken with one attenuator in the beam path. As a start, only the measured without attenuators are considered. Furthermore, the cells can be grouped into keratin-free cells, cells with a keratin network and cells with little keratin (see Fig. C.19 for an example).

Fig. 6.19 shows an overview of all power law exponents for fixed-hydrated SK8/18-2 cells measured at the P10 beamline without attenuators. The data points are arranged in four different groups, i.e. power law exponent obtained in the low and high $q_{r}$-region for the cell body and the nucleus, respectively. Further, in each group the data points in the first column corresponds to cells with a keratin network ( 5 cells analyzed), the data points in the second column corresponds to cells with little keratin content (3 cells analyzed) and the data points in the third column corresponds to cells without keratin ( 2 cells analyzed). Taking into account the spreading of the data points for cells of the same kind (keratin-free, high or low keratin content) and the low number of measured cells, no difference between the three cell kinds can be observed in the power law exponents. Therefore the average structures in the different cell kinds, as probed by the averaged scattering patterns, are too similar to allow for a differentiation of the cells.

The power law exponents for all measured fixed-hydrated cells were averaged taking into account the position on the cell, i.e. the nucleus or the cell body, and the fit region. The resulting mean power law exponents and the standard deviation of the mean presented in Tab. 6.4. For averaging the "normal" average and not the "weighted" average with regard to the error was used, because the errors only 


\begin{tabular}{lc}
\hline & mean power law exponent \\
\hline nucleus, low $q_{r}$-values & $-3.27 \pm 0.05$ \\
nucleus, high $q_{r}$-values & $-3.88 \pm 0.04$ \\
cell body, low $q_{r}$-values & $-3.14 \pm 0.05$ \\
cell body, high $q_{r}$-values & $-3.75 \pm 0.06$ \\
\hline
\end{tabular}

Tab. 6.4.: Mean power law exponents and standard deviation of the mean for all fixedhydrated SK8/18-2 cells measured at the P10 beamline. (P10, Oct. 2012 and March 2013)

include the goodness of the fits and no information about the accuracy of the measurement or the state of the measured cell. Both, for the low and high $q_{r}$-region, the power law exponents for the cell body are slightly higher than for the nucleus. However, due to the low number of analyzed cells, this small difference between the nucleus and the cell body could also be an artifact.

\subsubsection{Influence of Fitting Range}

The comparison of the power law exponents for all the fixed-hydrated cells raised the question to which extend the selected $q_{r}$-range for fitting influences the power law exponents. To estimate this effect, fits using four different $q_{r}$-ranges as displayed in Fig. 6.20 were performed and also extreme and obviously unsuited conditions as employed in Fig. 6.20a were tested. The resulting power law exponent are presented in Fig. 6.21 as red data points along with the exponents for all fixed-hydrated cells plotted in gray and black. All data points obtained for the fixed-hydrated cells are plotted in one column, since there was no difference observed for the different cells (keratin-free cells or cells with a high or low keratin content). The fits to the high $q_{r}$-region are very stable to changes of the employed $q_{r}$-range, showing only small variations of the exponents. For the fits to the low $q_{r}$-region, the power law exponents are more sensitive to the selected range. However, these exponents obtained for different fitting ranges stay approximately within the region that is spanned by the power law exponents for different cells. For the "visually good"

Fig. 6.20. (following page): Selection of different $q_{r}$-range for the determination of power laws exponents for fixed-hydrated cells. The $q_{r}$-ranges over which the fit functions are plotted correspond to the regions that were used for fitting. The $q_{r}$-range pixel numbers are given in each subfigure. (Sample BW139, position 3; P10, Oct. 2012) 


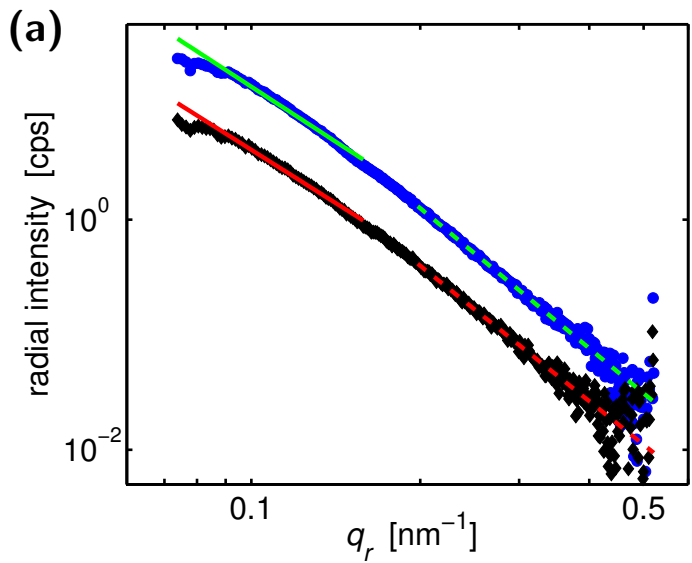

fit range 1: pixel $56-120$

fit range 2: pixel 150-395

- ROI 1 - ROI 3 (nucleus)

- ROI 2 - ROI 3 (cell body)

- fit $f(q)=9.51 \mathrm{e}-03^{*} q^{-3.18}+9.22 \mathrm{e}-10$

- fit $\mathrm{f}(q)=3.37 \mathrm{e}-03^{*} q^{-3.08}+1.18 \mathrm{e}-12$

- - fit $f(q)=1.88 \mathrm{e}-03^{*} q^{-4.04}+2.01 \mathrm{e}-10$

- - fit $\mathrm{f}(q)=7.35 \mathrm{e}-04^{*} q^{-3.91}+3.13 \mathrm{e}-09$

(b)

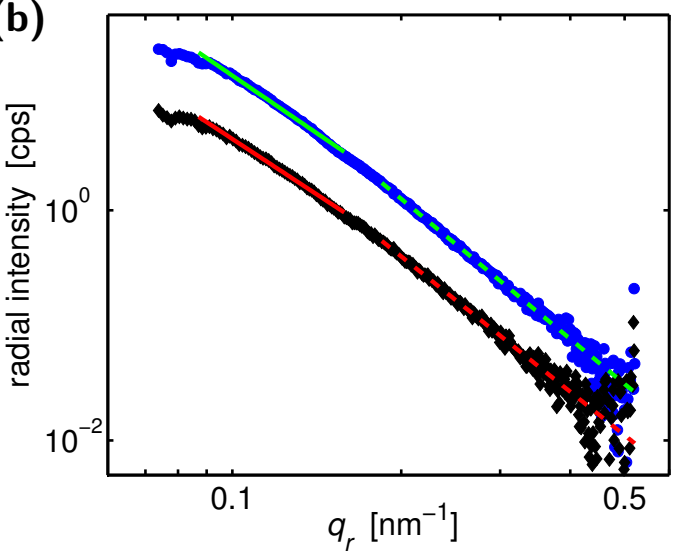

fit range 1: pixel $66-120$

fit range 2: pixel 140-395

- ROI 1 - ROI 3 (nucleus)

- ROI 2 - ROI 3 (cell body)

fit $f(q)=6.97 \mathrm{e}-03^{*} q^{-3.33}+2.46 \mathrm{e}-10$

- fit $\mathrm{f}(q)=2.62 \mathrm{e}-03^{*} q^{-3.20}+2.75 \mathrm{e}-10$

- - fit $\mathrm{f}(q)=1.87 \mathrm{e}-03^{*} q^{-4.04}+2.45 \mathrm{e}-10$

- - fit $f(q)=7.39 \mathrm{e}-04^{*} q^{-3.91}+2.62 \mathrm{e}-09$

(c)

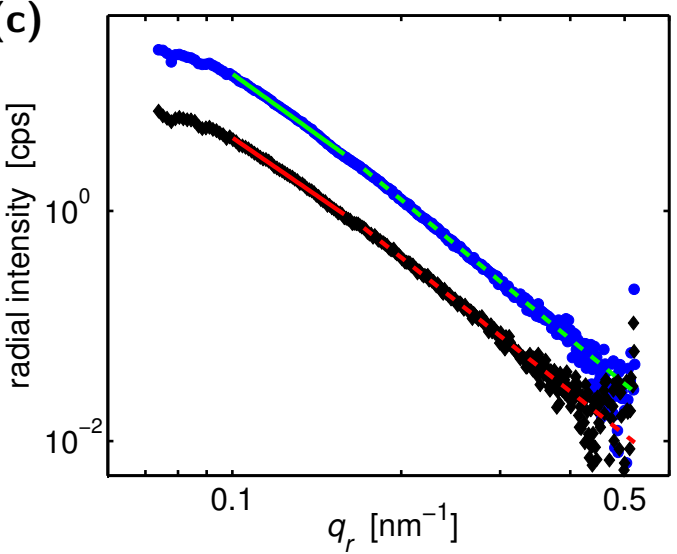

fit range 1: pixel 76-120

fit range 2: pixel 130-395

- ROI 1 - ROI 3 (nucleus)

- ROI 2 - ROI 3 (cell body)

- fit $\mathrm{f}(q)=4.85 \mathrm{e}-03^{*} q^{-3.51}+3.73 \mathrm{e}-12$

- fit $\mathrm{f}(q)=1.97 \mathrm{e}-03^{*} q^{-3.34}+6.64 \mathrm{e}-09$

- - fit $\mathrm{f}(q)=1.93 \mathrm{e}-03^{*} q^{-4.02}+3.13 \mathrm{e}-11$

- - - fit $\mathrm{f}(q)=7.80 \mathrm{e}-04^{*} q^{-3.87}+3.00 \mathrm{e}-09$

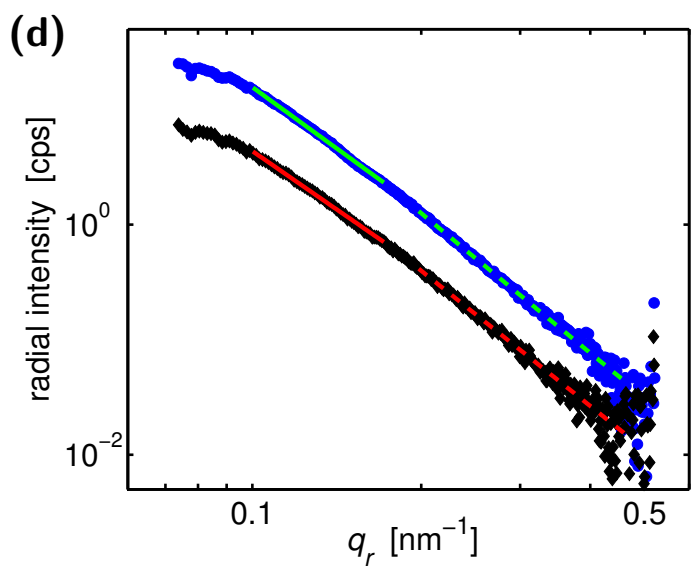

fit range 1: pixel 76-130

fit range 2: pixel 150-350

- ROI 1 - ROI 3 (nucleus)

- ROI 2 - ROI 3 (cell body)

fit $f(q)=4.51 \mathrm{e}-03^{*} q^{-3.54}+2.49 \mathrm{e}-08$

一 fit $\mathrm{f}(q)=1.84 \mathrm{e}-03^{*} q^{-3.37}+3.63 \mathrm{e}-09$

- - fit $\mathrm{f}(q)=1.87 \mathrm{e}-03^{*} q^{-4.04}+6.15 \mathrm{e}-04$

- - fit $f(q)=7.41 \mathrm{e}-04^{*} q^{-3.90}+2.58 \mathrm{e}-09$ 
choices of the fitting range in Fig. 6.20 b and $\mathrm{d}$ the exponents differ only by less than $1 \%$. Therefore severe artifacts or modification of the exponents due to slight variations of the fitting range can be excluded.

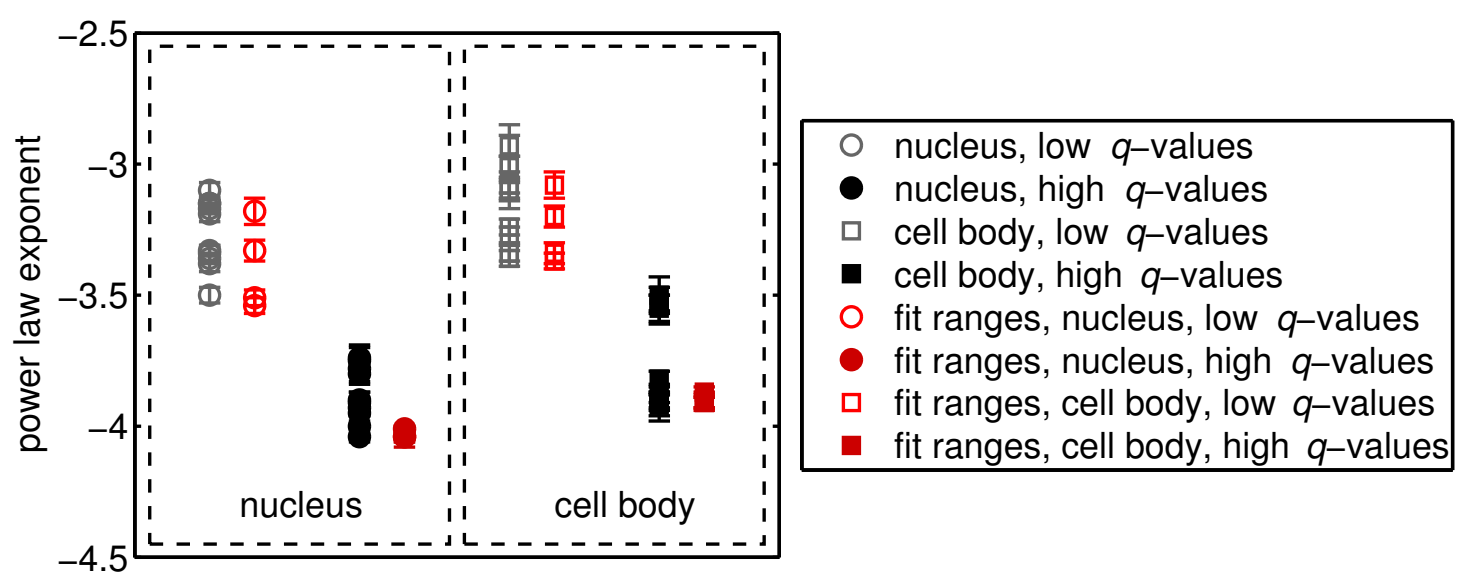

Fig. 6.21.: Influence of the selected $q_{r}$-range for fitting on the determination of power laws exponents for fixed-hydrated cells. The red data points are the power law exponents obtained for the different fit-ranges shown in Fig. 6.20 and the black and gray data points are all power law exponents for the fixed-hydrated cell. The error bars indicate the errors obtained from the fits with a power law. (P10, Oct. 2012 and March 2013)

\subsubsection{Influence of Beam Attenuation}

To reduce the radiation damage on hydrated cells, some measurements were performed with one attenuator in the beam path (compare Tab. 6.3). These cells were analyzed using the same scheme as presented above. Fig. 6.22 shows the power law exponents from these measurements as green data points along with the exponents for the fixed-hydrated cells plotted in gray and black.

The power law exponents obtained in the low $q_{r}$-region are higher for measurements with an attenuator compared to measurements without attenuators. In the high $q_{r}$-region, the power law exponents obtained on the nucleus are slightly higher and the exponents obtained on the cell body are slightly smaller for measurements with an attenuator. Deviations might be attributed to the higher signal-to-noise ratio for measurements with an attenuator due to the lower primary beam intensity. This interpretation is supported by the finding that for freeze-dried samples, which show a much stronger scattering signal and therefore also a higher signal-to-noise ratio, no difference between the power law exponents obtained for measurements with or without an attenuator was observed (compare to the power law exponents obtained 


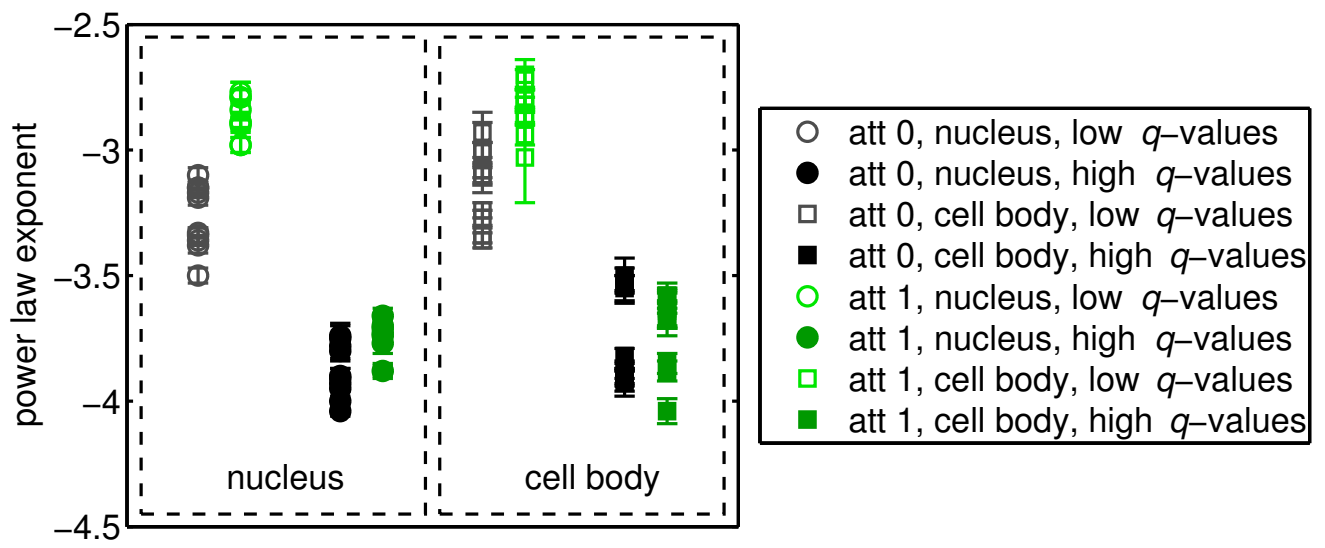

Fig. 6.22.: Influence of X-ray beam attenuation on the power laws exponents for fixedhydrated cells. The green data points correspond to measurements with one attenuator and the gray and black data points correspond to measurements without attenuators. The error bars indicate the errors obtained from the fits with a power law. (P10, Oct. 2012 and March 2013)

for freeze-dried cells at the P10 beamline in Fig. 5.21 and the scan parameters in Tab. 5.2).

\subsubsection{Radial Intensity of Single Scattering Patterns}

Single scattering patterns were integrated in azimuthal direction, in order to test whether information about the local sample structure can be extracted from single scattering patterns from fixed-hydrated cells. One example for a scattering pattern recorded on the nucleus is displayed in Fig. 6.23 b. The location of this position in the scan is marked in the X-ray dark-field image in Fig. 6.23a. The scattering pattern shows a weak scattering signal close to the beamstop. Since the nucleus is supposed to scatter strongest compared to other regions of the cell, further scattering patterns from other regions of the cell that scatter less, are not presented here. Similar to the measurements on freeze-dried samples at the P10 beamline, no scattering maxima are observed in the individual scattering pattern.

Azimuthal integration of the scattering pattern from the nucleus in one angular segment results in the radial intensity profile shown in Fig. 6.24. The red curve in this figure corresponds to the radial intensity profile of the averaged scattering patterns from the empty region as employed above (compare Fig. 6.16). Up to about $0.3 \mathrm{~nm}^{-1}$ the signal from the cell is well above the average background signal. Aside from the noise in the data, the radial intensity decays smoothly without peaks or shoulders. Since the scattering patterns from freeze-dried samples that were recorded at the 
(a)

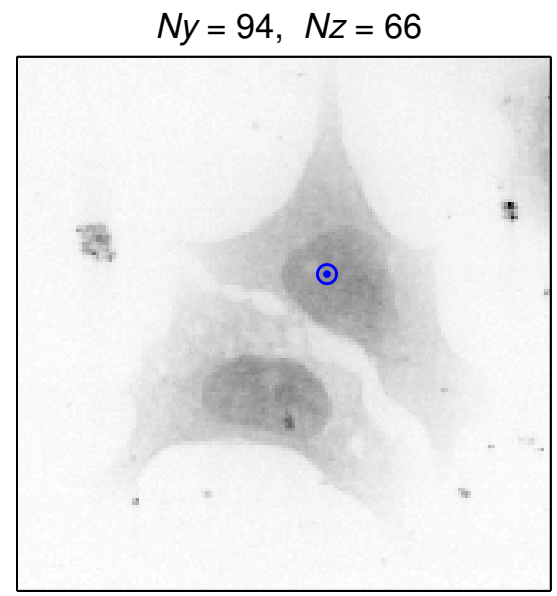

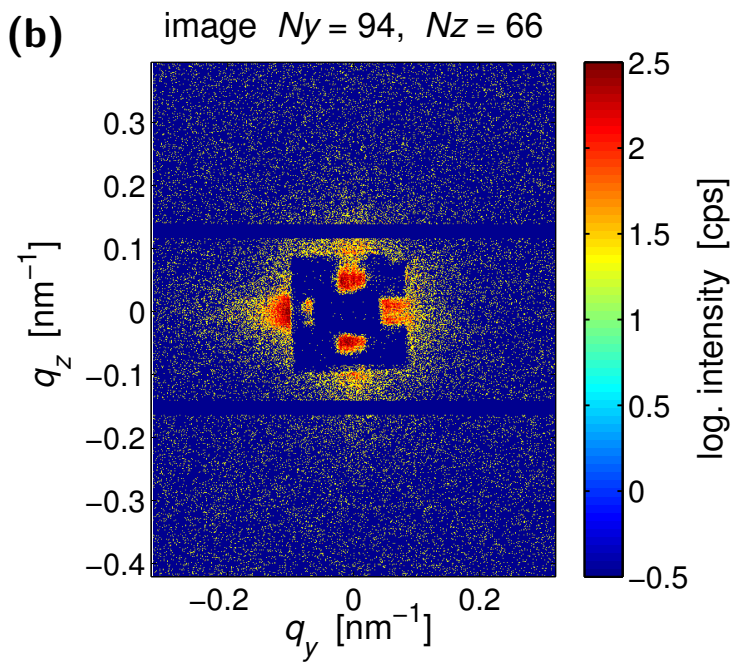

Fig. 6.23.: Single scattering pattern from the nucleus of a fixed-hydrated SK8/18-2 cell. (a) Location of the scattering pattern in the scan. (b) Single scattering pattern. (Sample BW235, position 1; P10, March 2013)

P10 beamline did not show scattering maxima or shoulders either, the same observation for fixed-hydrated cells can be partially attributed to the large beam size at P10 beamline. For fixed-hydrated cells, also the lower electron density contrast between the cellular material and the buffer solution affects the scattering signal. Subtraction of the background signal from the cell signal results in the profile displayed in Fig. 6.25. In analogy to the analysis of the averaged scattering patterns, the background corrected radial intensity of the single scattering patterns was fitted with two power laws in the low and high $q_{r}$-region, respectively. The obtained power law exponents are similar to the ones obtained for the averaged scattering signals.

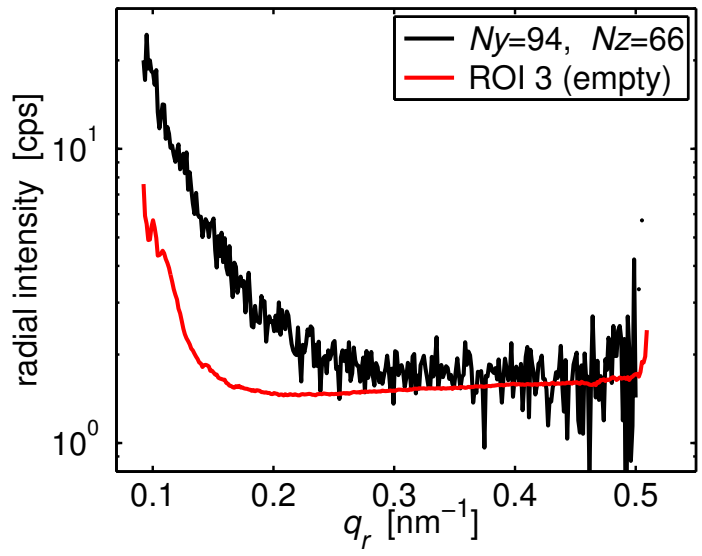

Fig. 6.24.: Radial intensity of a single scattering pattern from the nucleus of a fixedhydrated SK8/18-2 cell and averaged background signal as determined above. (Sample BW235, position 1; P10, March 2013) 


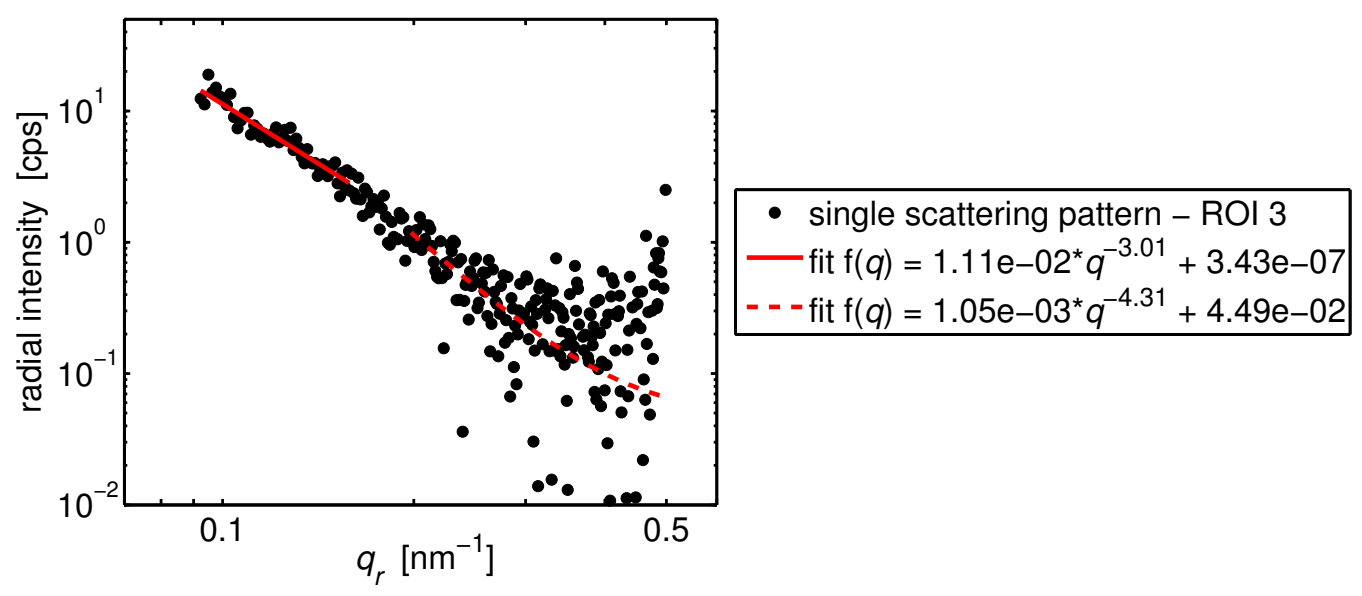

Fig. 6.25.: Fitting of two power laws to the low $q_{r}$-region and high $q_{r}$-region of the background corrected radial intensity profile of single scattering pattern. (Sample BW235, position 1; P10, March 2013)

Therefore, compared to the averaged scattering patterns, no additional information about the local sample structure can be obtained from the individual scattering patterns. 


\subsection{Specific Challenges}

Several specific problems occurred during the measurements of hydrated samples. In the following part some of these problems and general issues worth being considered shall be briefly explained and discussed. The values for the material properties employed for the following estimations were taken from different literature sources and were partially determined at different temperatures ranging from $20^{\circ} \mathrm{C}$ to $27^{\circ} \mathrm{C}$. However, effect of the different temperature values should be negligible.

\subsubsection{Radiation Damage}

For all synchrotron experiments damage to the sample due to the intense X-ray beam is a crucial issue. Particularly for the measurements on the hydrated samples this became obvious and damage to the cells could be observed with the beamline microscopes during the measurement or after the scan was completed.

Fig. 6.26a shows the visible light phase contrast microscopy image of two SK8/18-2 cells. The image taken with the on-axis beamline microscope before and after the measurement are displayed in Fig. 6.26b and c, respectively. Before the measurement the cells are hardly visible with the beamline microscope. After the measurement the cells appear whitish in the beamline microscope image and they can be clearly identified, which indicates structural modifications to the cells upon exposure to Xrays. The bright spots in Fig. $6.26 \mathrm{~b}$ and $\mathrm{c}$ that are not influenced by the radiation are dirt particles on the $\mathrm{Si}_{3} \mathrm{~N}_{4}$ membrane.

In severe cases, detachment of the measured cells from the substrate was observed, as shown in Fig. 6.27. Fig. 6.27a shows the visible light phase contrast microscopy image of a fixed-hydrated SK8/18-2 cell. A mesh scan was performed on the cell
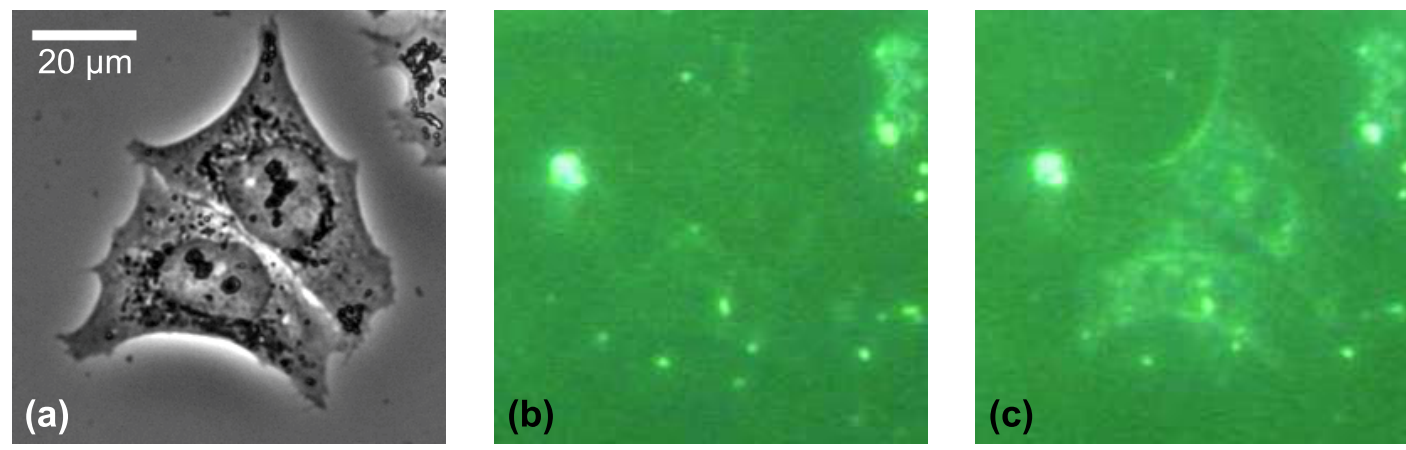

Fig. 6.26.: Radiation damage to fixed-hydrated SK8/18-2 cells during a measurement at the P10 beamline. (a) Visible light phase contrast microscopy image of two SK8/182 cells. (b) Image taken with the on-axis beamline microscope before and (c) after the measurements. (Sample BW235, position 1; P10, March 2013) 

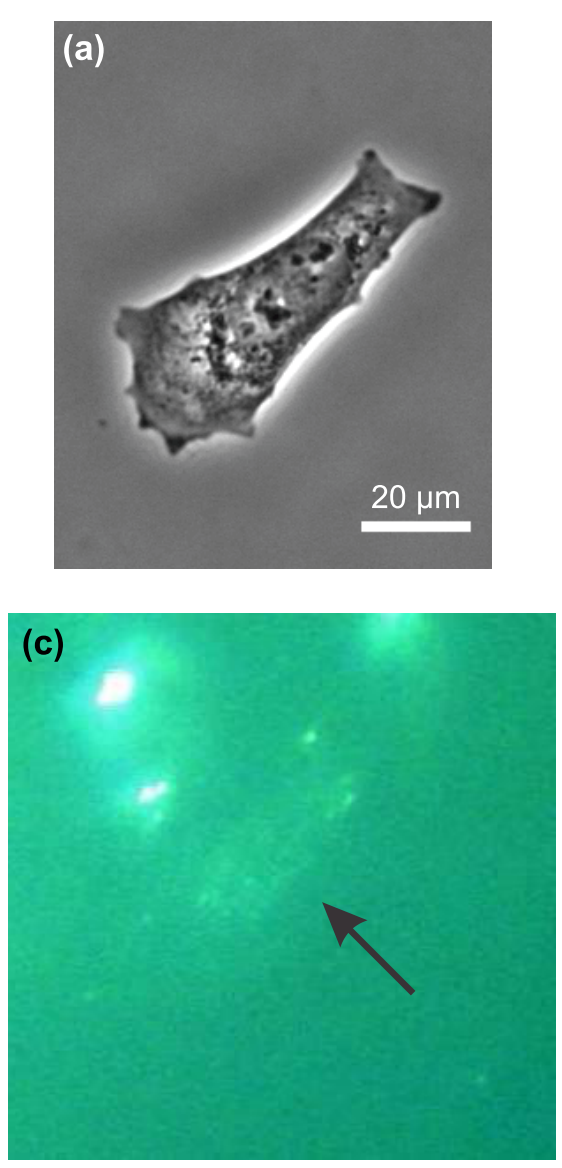
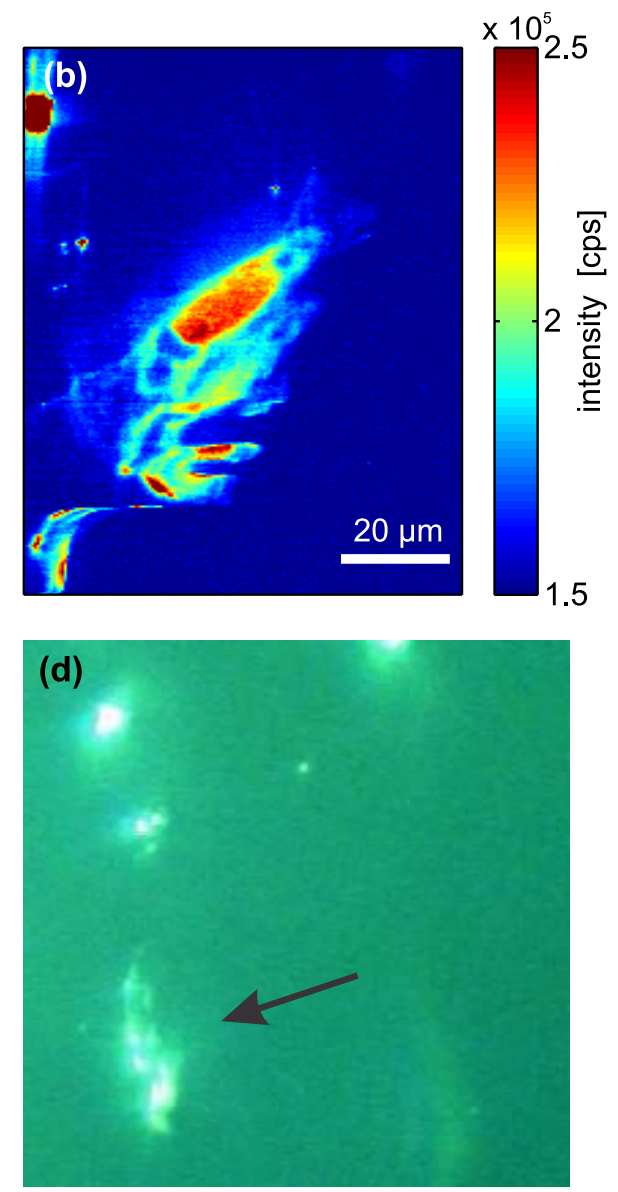

Fig. 6.27.: Detachment of a fixed-hydrated SK8/18-2 cell from the substrate during a measurement at the P10 beamline. (a) Visible light phase contrast microscopy image of a fixed-hydrated cell. (b) X-ray dark-field image of the same sample region as shown in panel (a). (c) Image taken with the on-axis beamline microscope before and (d) after the measurements. The arrows indicate the cell positions. (Sample BW143, position 3; P10, Oct. 2012)

and the corresponding X-ray dark-field image is presented in Fig. 6.27b. Here, the upper part of the cell can be clearly identified, but in the lower half of the image intensity streaks appear around the cell. The streaks in the lower half of the image result from the detached upper part of the cell, which floats in the solution. Note that the scan was performed from the left to the right side and from the top to the bottom of the image. Images of the same cell taken with the on-axis beamline microscope before and after the measurement are shown in Fig. 6.27c and d, respectively. The arrows indicate the positions of the cell. Before the measurement the cell is hardly visible, but after the measurement the destroyed remaining cell can be clearly identified. Cell detachment could be prevented by employing larger step sizes or by attenuation of the beam. 
Another visible consequence of the radiation damage to the sample was a low total scattered intensity in the X-ray dark-field images for measurements with small step sizes. This observation can be explained by the destruction of the sample structure before the actual measurement due to radicals that are created in the cell and in the buffer solution. This effect was particularly apparent for measurements at the ID13 beamline, due to the non-continuous and therefore slow scanning mode.

To further understand this effect, the mechanism of radiation damage to the cellular material needs to be understood. In hydrated cells, X-rays create ions, free radicals, free electrons and excited molecules, which then chemically react with the cellular material and produce damage [104,106]. Particularly, the hydroxyl radical is considered to be the major cause of damage [104, 106]. The diffusion constant of hydroxyl radicals in water, as obtained from molecular dynamics simulation, is $D_{\mathrm{HO}}=7.1 \times 10^{-9} \mathrm{~m}^{2} / \mathrm{s}[146]$, which results in a mean squared displacement of $\left\langle\mathbf{r}^{2}\right\rangle=6 D \tau=2130 \mu \mathrm{m}^{2}$ or $\sqrt{\left\langle\mathbf{r}^{2}\right\rangle}=46 \mu \mathrm{m}$ during one exposure of $0.5 \mathrm{~s}$ as employed at the ID13 beamline, with $D$ being the diffusion coefficient and $\tau$ the diffusion time. However, the average life time for hydroxyl radicals involved in a reaction with the cellular DNA was determined in reference [105] yielding the average diffusion distance of about $6 \mathrm{~nm}$ before reaction. Since also the buffer solution (or the medium for experiments on living cells) contains molecules other than water, e.g. phosphate (or amino acids and sugars), free diffusion of the hydroxyl radicals does probably not occur. By contrast, the created free electrons can, depending on their kinetic energy, penetrate several micrometers and create more radicals as well as free electrons along their way, which results in a spreading of the radiation induced damage. Furthermore, chemical reactions involving radicals are often chain reactions. Therefore the damage sites will spread within a protein due to chemical reactions, which is determined by the rate constants of the specific chemical reaction and should not be further estimated here due to the high variability, or within the whole cell due to diffusion of more stable radical species. The mean squared displacement of a small protein with a diffusion constant of about $10 \mu \mathrm{m}^{2} / \mathrm{s}$ (compare [147]) during one exposure of $0.5 \mathrm{~s}$ is about $\left\langle\mathbf{r}^{2}\right\rangle=30 \mu \mathrm{m}^{2}$ or $\sqrt{\left\langle\mathbf{r}^{2}\right\rangle}=5.5 \mu \mathrm{m}$, which might contribute to the damage spreading. However, since the radiation damage will only reach observable magnitudes in regions with a (critically) high radical concentration, only regions close to the exposed position will be (visibly) destroyed within short time intervals after the exposure. Therefore, by taking larger steps in one or both scan directions, the destroyed sample regions can be skipped resulting in a stronger scattering signal and a higher signal-to-noise ratio. However, for longer incubation times regions with a lower radical concentration will be visibly degraded as well and thus fast scanning is important for measurements on hydrated samples. 
Also for larger step sizes in vertical direction of $4 \mu \mathrm{m}$, as employed for living cells at the P10 beamline (compare section 7.3), the diffusion time for covering the line spacing becomes $0.1 \mathrm{~s}$. This is longer than a single exposure of $0.05 \mathrm{~s}$, but it is still one order of magnitude faster than the scan time per line, which is about $2.5 \mathrm{~s}$ for 50 points per line.

\subsubsection{Heat Input}

It is also interesting and illustrative to estimate the heat input into the sample or sample chamber during the measurement. For this calculation we assume that the total absorbed energy is transformed into heat, which gives the upper limit of the heat input. For the employed set-up parameters at the P10 beamline, i.e. a photon energy of $7.9 \mathrm{keV}$ and an primary beam intensity of $10^{11} \mathrm{cps}$, and the absorption of a $200 \mu \mathrm{m}$ water layer of $19 \%$ [148 the absorbed power is

$$
\Delta P_{\mathrm{abs}}=0.19 \times 7.9 \mathrm{keV} \times 10^{11} \mathrm{~s}^{-1}=2.4 \times 10^{-5} \mathrm{~J} / \mathrm{s}
$$

We can further estimate, to which extent the temperature of the liquid changes during a measurement. In an naive approach we can assume that the total energy loss per $0.05 \mathrm{~s}$ exposure is transferred to the volume that is probed by the beam, i.e. $V_{\text {probe }}=200 \mu \mathrm{m} \times 300 \mathrm{~nm} \times 300 \mathrm{~nm}=1.8^{-11} \mathrm{~cm}^{3}$ for a focal spot size of $300 \mathrm{~nm} \times$

$300 \mathrm{~nm}$. This would result in an enormous local temperature change $\Delta T_{\text {local }}$ of

$$
\Delta T_{\text {local }}=\frac{\Delta Q}{c_{p} m}=\frac{2.4 \times 10^{-5} \mathrm{~J} / \mathrm{s} \times 0.05 \mathrm{~s}}{4.187 \mathrm{~J} /(\mathrm{g} \mathrm{K}) \times 1.8 \times 10^{-11} \mathrm{~g}}=1.6 \times 10^{4} \mathrm{~K},
$$

with $\Delta Q$ being the transferred heat, $c_{p}$ the specific heat capacity of water at $27^{\circ} \mathrm{C}$ [128 and $m$ the mass of the heated water. However, there are several reasons for the absorbed energy to be not confined to the focal volume, but being distributed over a larger volume already during one exposure.

Firstly, at X-ray energies of around $10 \mathrm{keV}$ the most prominent process leading to Xray absorption will be the photoelectric effect, i.e. the absorption of a photon by an electron, which is then released from the atom. The penetration depth of electrons with a kinetic energy of around $10 \mathrm{keV}$ in matter is a few micrometers [149], meaning that also the energy will be dissipated within this range. Secondly, diffusion of water molecules and the collisions with other molecules will lead to an equilibration in the whole sample chamber. With a self-diffusion coefficient of $D_{\mathrm{H}_{2} \mathrm{O}}=2.025 \times 10^{-9} \mathrm{~m}^{2} / \mathrm{s}$ of water at $20^{\circ} \mathrm{C} 150$ the mean squared displacement within one exposure of $0.05 \mathrm{~s}$ is about $\left\langle\mathbf{r}^{2}\right\rangle=6 D \tau=608 \mu \mathrm{m}^{2}$ and $\sqrt{\left\langle\mathbf{r}^{2}\right\rangle}=25 \mu \mathrm{m}$. Thirdly, a continuous flow with a velocity of about $0.3 \mathrm{~mm} / \mathrm{s}$ in the channel is applied to the device leading to an average maximum displacement of $15 \mu \mathrm{m}$ of the fluid in the chamber within one 
exposure of $0.05 \mathrm{~s}$.

Assuming now, as the other extreme case, an equilibration in the whole sample chamber while neglecting the channel volumes as well as the flow through the device, a water volume of $\pi \times(1.75 \mathrm{~mm})^{2} \times 200 \mu \mathrm{m}=1.9 \times 10^{-3} \mathrm{~cm}^{3}$ needs to be considered. Furthermore, not only a single exposure, but the total mesh scan with, e.g. $101 \times 101$ scan points, is included. This leads to a temperature change of

$$
\Delta T_{\text {chamber }}=\frac{\Delta Q}{c m}=\frac{2.4 \times 10^{-5} \mathrm{~J} / \mathrm{s} \times 101 \times 101 \times 0.05 \mathrm{~s}}{4.187 \mathrm{~J} /(\mathrm{g} \mathrm{K}) \times 1.9 \times 10^{-3} \mathrm{~g}}=1.5 \mathrm{~K}
$$

per mesh scan. Taking into account the total time of the measurement of about 15 min, which allows for a heat exchange with the environment, and the continuous fluid flow through the devices or the sample chamber, the temperature change during one measurement should be negligible. However, it is still not obvious how the temperature in the chamber changes during one scan and how the superposition of the temperature changes from neighboring exposures affects the local temperature. To gain more insight into how the heat equilibrates laterally in the chamber (in the $y$ $z$-plane, with $x$ being the beam direction) and how the superposition of neighboring exposures affects the local temperature, the heat equation in two dimensions can be employed:

$$
\frac{\partial T(\mathbf{r}, t)}{\partial t}=\alpha \Delta T(\mathbf{r}, t)=\alpha\left(\frac{\partial^{2} T(\mathbf{r}, t)}{\partial x^{2}}+\frac{\partial^{2} T(\mathbf{r}, t)}{\partial y^{2}}\right)
$$

with $T$ being the time dependent temperature field in the chamber and $\alpha$ the thermal diffusivity of water ${ }^{1}$. In beam direction, the temperature is assumed to be the same for each fluid layer.

For solving of Eq. 6.4, appropriate initial conditions are needed. As discussed above, it is not easy to determine the temperature profile after a certain exposure time or during the exposure, because during the exposure the heat is continuously transferred to the water layer and the equilibration process starts directly upon interaction of the X-rays with the buffer. One approach to approximate the temperature profile is to use a two-dimensional box model with a temperature of $\Delta T_{\text {local }}$ as initial temperature distribution at $t=0 \mathrm{~s}$, which is then allowed to equilibrate for $50 \mathrm{~ms}$ or longer yielding the temperature distribution at the end of one exposure or at a later time point after the exposure, respectively. Since the heat equation is not solvable analytically for this initial condition, a Gaussian temperature distribution with a maximum value of $T_{0}=\Delta T_{\text {local }}$ and a full width at half maximum corresponding to

\footnotetext{
${ }^{1}$ The thermal diffusivity is defined by $\alpha=k /\left(c_{p} \rho\right)$, with $k=0.60 \mathrm{~W} /(\mathrm{m} \mathrm{K})$ being the thermal conductivity at $25^{\circ} \mathrm{C}, c_{p}=4187 \mathrm{~J} /(\mathrm{kg} \mathrm{K})$ the specific heat capacity at $27^{\circ} \mathrm{C}$ and $\rho=1 \mathrm{~g} / \mathrm{cm}^{3}$ the density of water a value of $\alpha=1.43 \times 10^{-7} \mathrm{~m}^{2} / \mathrm{s}$ is obtained 128 .
} 
the beam size, i.e. $\sigma=300 \mathrm{~nm} /(2 \sqrt{2 \log 2})$, is used

$$
T(\mathbf{r}, 0)=T_{0} \exp \left(-\frac{\mathbf{r}^{2}}{2 \sigma^{2}}\right) \text {. }
$$

However, it should be kept in mind that the initial condition at $t=0$ is not realized in the physical system. Solving the heat equation with this initial condition yields a lateral temperature field in the chamber of

$$
T(\mathbf{r}, t)=\frac{T_{0} \sigma^{2}}{\sigma^{2}+2 \alpha t} \exp \left(-\frac{\left(x-x_{0}\right)^{2}+\left(y-y_{0}\right)^{2}}{2\left(\sigma^{2}+2 \alpha t\right)}\right)
$$

Using Eq. 6.6, the temperature fields resulting from each individual exposure can be calculated using different time points $t_{n}=n \Delta t, n \in \mathbb{N}$, and center positions $x_{0}$ and $y_{0}$. Furthermore, the superposition of the temperature fields resulting from different exposures in a scan can be determined. Fig. $6.28 \mathrm{a}$ shows the temperature increase for each individual exposure at the end of a line scan with 101 points, a step size of $0.5 \mu \mathrm{m}$ and a time interval of $\Delta t=0.05 \mathrm{~s}$ between two exposures. Here, the fluid flow through the device is neglected. The highest profile corresponds to the last scan point. The maximum temperature increase is less than $0.02 \mathrm{~K}$. However, the sum of all profiles from the same line scan, depicted in Fig. 6.28 b, shows a maximum temperature of about $0.1 \mathrm{~K}$. The scaling of the $y$-axis in both plots is chosen such that it covers the whole microfluidic chamber.

(a)

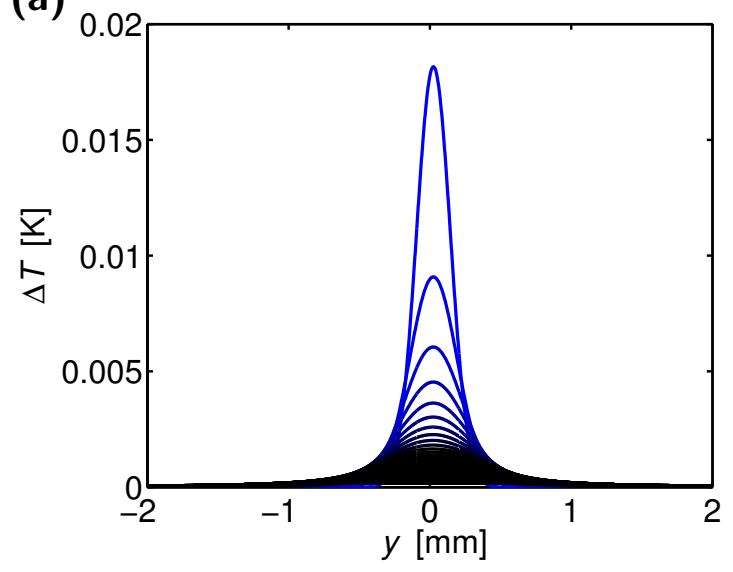

(b)

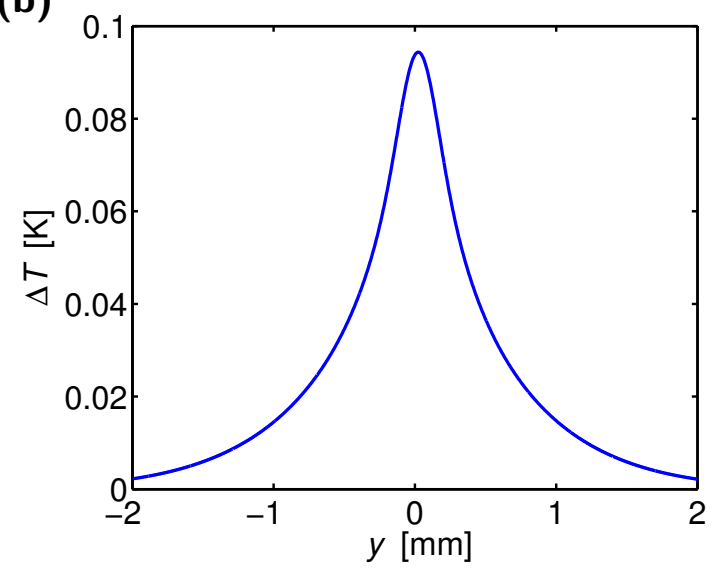

Fig. 6.28.: Temperature in the sample chamber during a line scan in $y$-direction with 101 points, a step size of $0.5 \mu \mathrm{m}$, a time interval of $0.05 \mathrm{~s}$ between two exposures and without a fluid flow through the device. (a) Temperature increase for each individual exposure at the end of the line scan. The lateral shift of $0.5 \mu \mathrm{m}$ between two consecutive exposures is not visible due to the scaling of the $y$-axis. (b) Sum of all temperature profiles shown in panel a. 

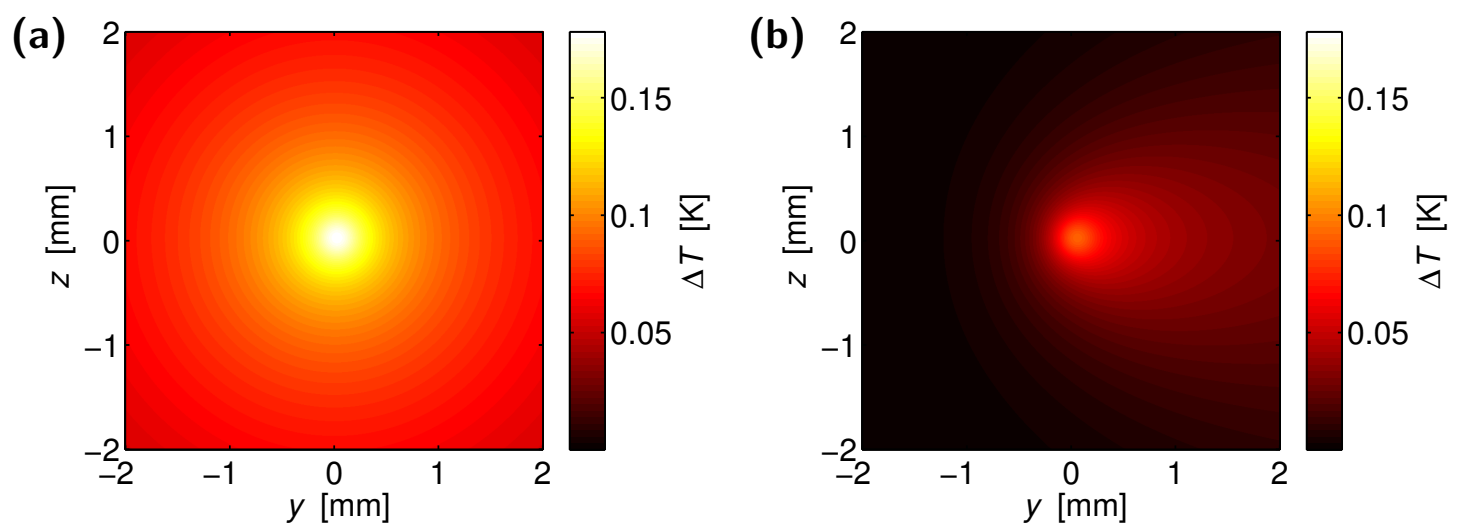

Fig. 6.29.: Temperature in the sample chamber directly after a mesh scan with $101 \times 101$ points, a step size of $0.5 \times 0.5 \mu \mathrm{m}^{2}$ and a time interval of $0.05 \mathrm{~s}$ between two exposures. (a) Sum of all temperature fields for the mesh scan without fluid flow. (b) Sum of all temperature fields for the mesh scan with an applied fluid flow in $y$-direction. The temperature scaling is chosen identically for both plots.

A similar consideration can be applied for mesh scans. The two-dimensional temperature maps after the last exposure of a mesh scan with $101 \times 101$ points, a step size of $0.5 \times 0.5 \mu^{2}$, a time interval of $0.05 \mathrm{~s}$ between two exposures and without fluid flow through the device is displayed in Fig. 6.29a. Here, the maximum temperature increase is about $0.18 \mathrm{~K}$, which is well below the estimate $\Delta T_{\text {chamber }}=1.5 \mathrm{~K}$ calculated above. The difference can be explained by the fact that for the calculation of $\Delta T_{\text {chamber }}$ only the (closed) volume in the chamber was considered, whereas an infinite water layer is assumed for solving of the heat equation. Fig. 6.28b shows that the temperature profile exceeds well beyond the chamber walls, which would be located at $\pm 1.75 \mathrm{~mm}$, and therefore heat would be transferred to the microfluidic device in the experiment.

The effect of a fluid flow through the devices in $y$-direction can be estimated by shifting the center of the temperature field by $\Delta y=15 \mu \mathrm{m}$ for each exposure. Fig. 6.29b shows the temperature map for the same mesh scan as in Fig. 6.29a, but with a flow through the device. Here, the maximum temperature is shifted in $y$-direction and it is about $50 \%$ lower than without flow.

As a conclusion from the considerations presented above and taking further the total time of the measurement of about 15 min into account, which allows for a heat exchange with the environment, the local and global temperature change during a measurement with the scan parameters as chosen above should be below $2 \mathrm{~K}$ and therefore negligible. However, when taking longer exposures per point, small step sizes or using sealed chamber without a continuous flow, the local temperature change might be higher and harmful for living cells. 


\subsubsection{Buffer Degassing}

Gas bubble formation or increase in the sample chamber during the measurement was another important issue. With the final method for buffer degassing, using a filtration unit that was places in an ultrasonic bath, very good results were obtained $:^{2}$ However, gas bubbles in the tubings or the syringes, which occurred during filling of the syringes and might be transported into the sample chamber, cannot be completely avoided.

\footnotetext{
${ }^{2}$ This protocol was suggested by Marten Bernhardt and Marius Priebe.
} 


\subsection{Summary and Closing Discussion}

Microfluidic devices or sandwich wet chambers were employed as sample environment for the fixed-hydrated cell (compare section 4.3 and [33, 40]). Both types of sample environment worked well in terms of leakage and positional stability. In particular, we did not observe nano-scale vibrations of the set-up induced by the syringe pumps used for the microfluidic devices, even though the pumps were placed on the same table as the set-up. The formation or increase of gas bubbles in the sample chamber during the X-ray measurements could be avoided by degassing and sonicating the buffer, but remaining gas bubbles in the tubings or syringes, which occur during filling of the syringe, cannot be completely avoided.

When deciding which type of sample environment should be used for a certain experiment, several aspects need to be considered. On the one hand spatial constrictions of the specific set-up need to be considered. For example the microfluidic devices including the tubings might be too thick in beam direction. Additionally, it is more complicated and time-consuming to fabricate and assemble the microfluidic devices compared to the sandwich wet chambers. On the other hand, only microfluidic devices provide the possibility to apply a flow to the device, which cools the specimen and, in case of living cells, supplies the cells with nutrients and allows for chemical manipulation. For longer experiments on living cells in sealed environments without a flow through the device, bystander cell killing of non-irradiated cells might become an important issue [113. Furthermore, since microfluidic devices provide a more versatile sample environment, which can be easily adapted to specific requirements, optimizing this technique is worthwhile without being necessary for a certain experiment.

In the X-ray dark-field images recorded at the P10 beamline, the cell contours, the nuclei and substructures in the nuclei and the cell bodies could be clearly identified. Here a minimum step size of $500 \mathrm{~nm}$ was employed. At the ID13 beamline, the scattering signal was lower than at the P10 beamline, which resulted in more noise in the X-ray dark-field images. The cells could be identified in the X-ray dark-field images, but for some regions in the cell body the signal was just above the background regions. The lower scattering signal obtained at the ID13 beamline can be attributed on the one hand to the lower primary beam intensity and on the other hand to the higher X-ray photon energy ( $\approx 15 \mathrm{keV}$ compared to $7.9 \mathrm{keV})$, which results in a lower scattering cross-section for the interaction of X-ray photons with matter. At the cSAXS beamline the background scattering was so strong that no scattering signal from the hydrated cells could be observed. Improvements of the set-up in terms of background reduction would be necessary for further nanodiffraction experiments. Concluding from this observation, a high photon flux and 
low background scattering is needed to be able to record the scattering signal, as employed here for the generation of X-ray dark-field images, from hydrated cells. However, imaging of the cells using differential phase contrast was possible at the cSAXS beamline, since the beamstop could be removed without saturation of the detector. Here, a real space resolution in the order of the beam size (about $200 \mathrm{~nm}$ ) could be obtained and network-like substructure could be identified in the cell periphery. These substructures do, however, not correspond to the keratin network, since both, keratin-free and keratin-containing cells, showed similar substructures. For imaging of hydrated cells using X-rays, other methods like ptychographic, holographic or coherent diffractive imaging [10, 12, 28, 95, 137] could be employed and optimized for this sample type as well, and would probably yield better results in terms of real space resolution. Test experiments with SK8/18-2 cells in microfluidic devices (results not shown) proofed the feasibility of waveguide-based holographic imaging of hydrated cells. $3^{3}$ Applying ptychographic imaging on fixed-hydrated SK8/18-2 cells in wet chambers at the cSAXS beamline (May 2012) was not successful, which is probably related to the fact that no scattering signal could be detected from the fixed-hydrated cells due to the strong background scattering. However, at the ID13 beamline and at the P10 beamline the scattering signal from the hydrated cells could be detected and therefore also ptychographic image reconstruction should succeed. The radial intensity profiles from averaged scattering patterns were determined for the measurements at all three set-ups. The best signal-to-noise ratio was obtained for measurements at the P10 beamline. The radial intensity profiles recorded at the ID13 beamline were more noisy than at the P10 beamline, but a larger $q_{r}$-range could be recorded due to the different photon energy and sample-to-detector distance, and signal up to about $1 \mathrm{~nm}^{-1}$ could be observed. Therefore, at the P10 beamline, measurements on hydrated cells using a higher photon energy would be worth trying in order to record a larger $q_{r}$-range at the fixed sample-to-detector distance.

The power law exponents, obtained from fits to the background corrected radial intensity profiles recorded at the P10 beamline, have similar values for cells without keratin and cells with a high or low keratin content in the cytoplasm. Therefore, similar to the measurements on freeze-dried cells presented in chapter 5, it is not possible to distinguish between cells with or without keratin from the intensity decay of the averaged scattering patterns. For the hydrated cells, a small difference between the power law exponents recorded on the cell body and on the nucleus was observed, which could either represent the different average structure in those two

\footnotetext{
${ }^{3}$ Measurements at the P10 beamline and data analysis were performed by Matthias Bartels and Martin Krenkel.
} 


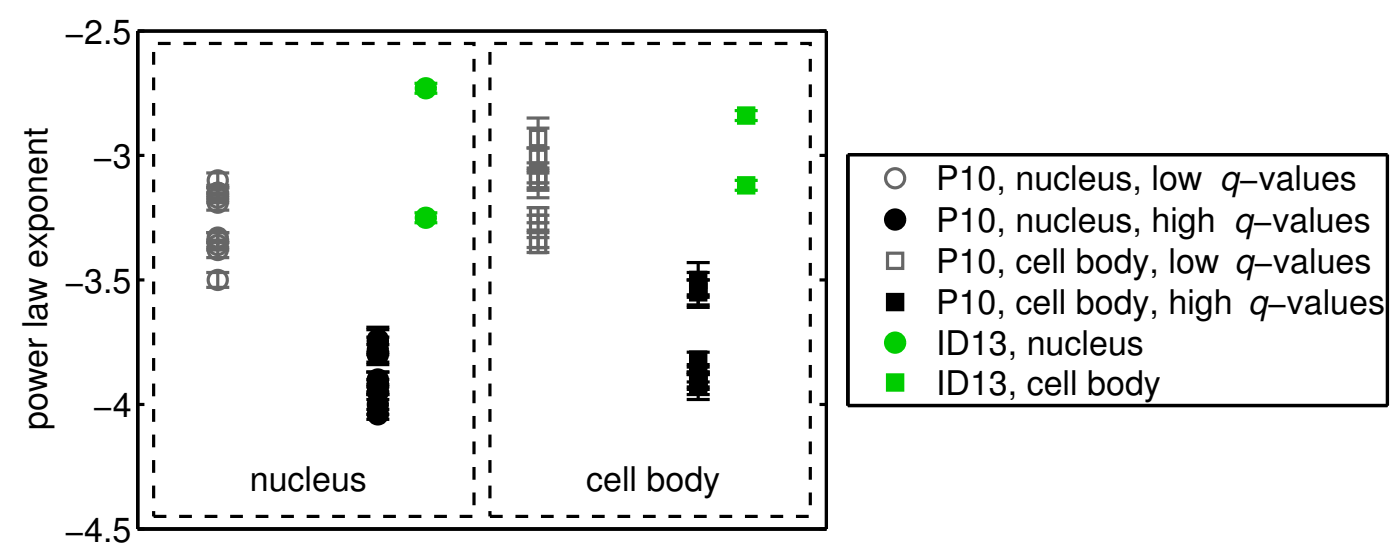

Fig. 6.30.: Summary of all power law exponents obtained from fits to the radial intensity profiles of averaged scattering patterns recorded on fixed-hydrated SK8/18-2 cells. Data points obtained at the P10 beamline are depicted in gray and black and at the ID13 beamline in green.

compartments or it could be attributed to an artifact due to the low number of analyzed cells. Here, measurements on more cells are needed to improve the data statistics. Furthermore, the phase of each cell in the cell division cycle is likely to influence the scattering signal, since, e.g., the chromatin condenses into the chromosomes during prophase or the keratin network disassembles during mitosis [2,61, 151]. In this context, a synchronization of the cell cycle would on the one hand allow for measurements of the cells in the same phase of the cell cycle and on the other hand to probe structural differences in cells that are in different phases. As also discussed for freeze-dried cell, measurements on cells from other cell lines could help to find characteristic power law exponents for certain cellular compartments or cell types. A summary of all power law exponents obtained for fixed-hydrated SK8/18-2 cells at the P10 beamline and at the ID13 beamline is presented in Fig. 6.30. The absolute values of the power law exponent obtained at the P10 beamline are in range of about -3.0 to -3.5 in the low $q_{r}$-region and in range of about -3.5 to -4.1 in the high $q_{r}$-region. The employed $q_{r}$-range at the ID13 beamline corresponds to the high $q_{r}$-range for the P10 data. However, the power law exponents obtained at the ID13 beamline are much higher than the exponents obtained at the P10 beamline in the high $q_{r}$-range. This deviation is probably due to the lower signal and the stronger noise for the data recorded at the ID13 beamline.

As discussed also for freeze-dried cells, corresponding to the well-known Porod law 142, 143, compact two-phase systems with a sharp interface exhibit a power law decay with $b=-4$, which fits very well to the intensity decay in the high $q_{r}$-region. 
However, as also mentioned earlier, the Porod law is commonly used to describe SAXS experiments, where the employed beam size is large compared to the probed structure sizes, while the employed beam size for the measurements presented here is in the same order of magnitude as the structures in the cells. Therefore, for the study of fixed-hydrated cells, measurements with a larger beam with a size of a few micrometers could be performed in order to study the effect of the beam size on the obtained power law exponents.

The radiation damage to the cells was stronger for the fixed-hydrated than for the freeze-dried samples, which is probably due to the higher concentration of radicals that are created during the X-ray illumination in the surrounding buffer (fixedhydrated cells) than in the surrounding air (freeze-dried cells). To reduce the destruction of the sample, radical scavengers could be added to the buffer solution. A calibrated beamline microscope with a higher resolution and the option to use fluorescence microscopy would further allow for precise positioning of single exposures on specific local cellular structure like keratin bundles. Using this approach, the destruction of the local structures by radicals created during previous exposures of a mesh scan, could be minimized. Since single scattering patterns from fixed-hydrated cells recorded at the P10 beamline or the ID13 beamline showed an isotropic scattering signal without scattering maxima or minima, a higher photon flux is needed to probe the structure of the hydrated cellular material at the nano-scale. Therefore, this type of experiment should ideally be performed at FEL, which provides a much higher photon intensity in a short time pulse and thereby allows for a higher resolution of the scattering patterns in reciprocal space. However, among several others challenges, the precise localization of the sample in the FEL beam as well as the high sample throughput are critical issues.

The comparison of the results from measurements on fixed-hydrated samples at the three different set-ups shows that a high photon flux as well as low background scattering is needed to be able to record the scattering signal from hydrated cells. From the employed beamlines, best scattering data on hydrated samples were obtained at the P10 beamline, because the high photon flux in combination with the continuous scanning mode allowed for fast measurements with a good signal-to-noise ratio. However, particularly for experiments on weakly scattering samples, further effort should be put into the optimization of this set-up in terms of cleanliness of the beam profile. Despite the lower photon flux at the ID13 beamline, the smaller beam size might be advantageous for experiments requiring a small probed volume. 



\section{Scanning Nano-Diffraction on Living Cells}

In this chapter, scanning X-ray nano-diffraction experiments on living SK8/18-2 cells are described. Living cells were measured at two different synchrotron radiation facilities and the results are presented and discussed. Since the signal-to-noise ratio for the measurements on fixed-hydrated cells shown in chapter 6 was much better at the P10 beamline than at the ID13 beamline, the radial intensity profiles for the living cells are only shown for the measurements at the P10 beamline. Parts of this chapter have been prepared for publication [41].

\subsection{Experiment}

The experiments were carried out at two different end-stations at different synchrotron radiation facilities: the ID13 beamline (ESRF) and the P10 beamline (PETRA III). The different set-ups and the specific experimental parameters during each beamtime are described separately in section 3.2 .

The cells were grown on $\mathrm{Si}_{3} \mathrm{~N}_{4}$ membrane windows in Göttingen and transported to the synchrotrons in microtubes filled with culture medium (compare section 4.2.2). At the beamlines, the cells were either stored in these tubes at room temperature until the measurement (ID13, November 2012) or the windows were taken out of the tubes and kept in dishes with culture medium (DMEM with $10 \%$ FCS and PenStrep) in a cell incubator at $37^{\circ} \mathrm{C}$ in a water saturated atmosphere with $5 \% \mathrm{CO}_{2}$ (P10, March 2013). The first method has the advantage that the required equipment can be reduced to a minimum and in particular no cell culture lab is needed. The cells could be kept in the sealed tube at room temperature for about two days without major morphological changes and even after four days cells with a normal morphology could be identified. However, the cells did not proliferate, indicating a negative effect of the non-optimum culture conditions. Keeping the living cells in an incubator at normal growth conditions, as it was possible for the measurements at the P10 beamline, is ideal since well-defined conditions for cells growth were obtained. 
For all experiments on living cells, microfluidic devices were used as sample environment. Bright-field microscopy images of the cells were taken and the microfluidic devices were assembled directly before the measurement by incorporation of the $\mathrm{Si}_{3} \mathrm{~N}_{4}$ membrane window with the cells as described in section 4.3.2. Tubing and a Hamilton Gastight glass syringe filled with degassed culture medium were attached to the microfluidic device and the device was connected to syringe pumps (neMESYS, Cetoni GmbH, Korbußen, Germany). At the P10 beamline, $\mathrm{CO}_{2}$ independent medium with 10\% FCS and Pen-Strep was used and the glass syringe with the liquid was warmed to $37^{\circ} \mathrm{C}$. During the measurement the device and the syringe were kept at room temperature. The buffer system in this medium is optimized for a $\mathrm{CO}_{2}$-free atmosphere and therefore the $\mathrm{pH}$ is kept constant in the "normal" atmosphere with $0.04 \% \mathrm{CO}_{2}$. However, this medium is commonly not used for cell culture, but only during transport of the cells. At the ID13 beamline, DMEM with 10\% FCS and Pen-Strep at room temperature was used, which is the standard medium employed for culture of these cells. However, to maintain the $\mathrm{pH}$ at physiological conditions, a concentration of $5 \% \mathrm{CO}_{2}$ in the atmosphere is needed and particularly after degassing of the medium, the $\mathrm{pH}$ did change to more basic conditions.

The device was mounted on a sample stage using a magnetic sample holder as shown on the photograph in Fig. 6.2 in chapter 6 and a small container for the liquid that was flushed through the devices was attached to the sample stage. Culture medium was pumped through the device at a constant flow rate of $100-200 \mu \mathrm{l} / \mathrm{h}$, corresponding to a mean flow velocity of about $0.5 \mathrm{~mm} / \mathrm{s}$. All living samples were mounted in such a way that the membrane on which the cells were grown was located on the downstream-side of the microfluidic device. Therefore, X-ray photons that were scattered from the cells did not pass through the water layer in between the two $\mathrm{Si}_{3} \mathrm{~N}_{4}$ membranes before reaching the detector. The samples were aligned in the focus of the X-ray beam using a calibrated microscope and two dimensional mesh scans were performed on the cells. Best results were obtained using asymmetric scans with a larger step size in vertical direction (slow scan axis) than in the horizontal direction (fast scan axis). This strategy reduces the total scan time and allows for skipping regions of the cell to which radicals, that were created in the last scan line, have already spread (compare the discussion in section 6.5). Due to the high radiation dose per scan, the cells die during the measurements, meaning that all measurements are performed on "initially living cells". 


\subsection{Results from Measurements at the ID13 Beamline}

In the following section, one example for measurements and results on initially living cells at the ID13 beamline is presented. An overview of the employed scan parameters and the estimated average radiation dose per step for this measurement is shown in Tab.7.1.

\begin{tabular}{lllllll}
\hline sample & set-up & $\Delta_{y, z}\left[\mu \mathrm{m}^{2}\right]$ & $N_{y} \times N_{z}$ & $T[\mathrm{~s}]$ & $D[\mathrm{~Gy}]$ \\
\hline Live Cells II.6, pos. 2 & Nov. 2012 & $1 \times 1$ & $21 \times 41$ & 0.5 & $1.6 \times 10^{6}$ \\
\hline
\end{tabular}

Tab. 7.1.: Summary of the scan parameters and the estimated average radiation dose per step for the measurement on living cells at the ID13 beamline. Here, $\Delta_{y, z}$ are the lateral step sizes, $N_{y}$ and $N_{z}$ the number of scan points in $y$ - and $z$-direction, $T$ is the exposure time and $D$ the estimated average radiation dose per step. The measurement was performed without attenuators in the beam path.

Fig. 7.1 a shows a bright-field microscopy image of two SK8/18-2 cells, which was taken directly before the $\mathrm{Si}_{3} \mathrm{~N}_{4}$ window with the living cells was incorporated in the microfluidic device. A mesh scan was performed on the marked region and the corresponding X-ray dark-field image is shown in Fig. 7.1 b. For the calculation of the X-ray dark-field image, the same detector mask as used for the fixed-hydrated cells was employed (compare Fig. 6.9).
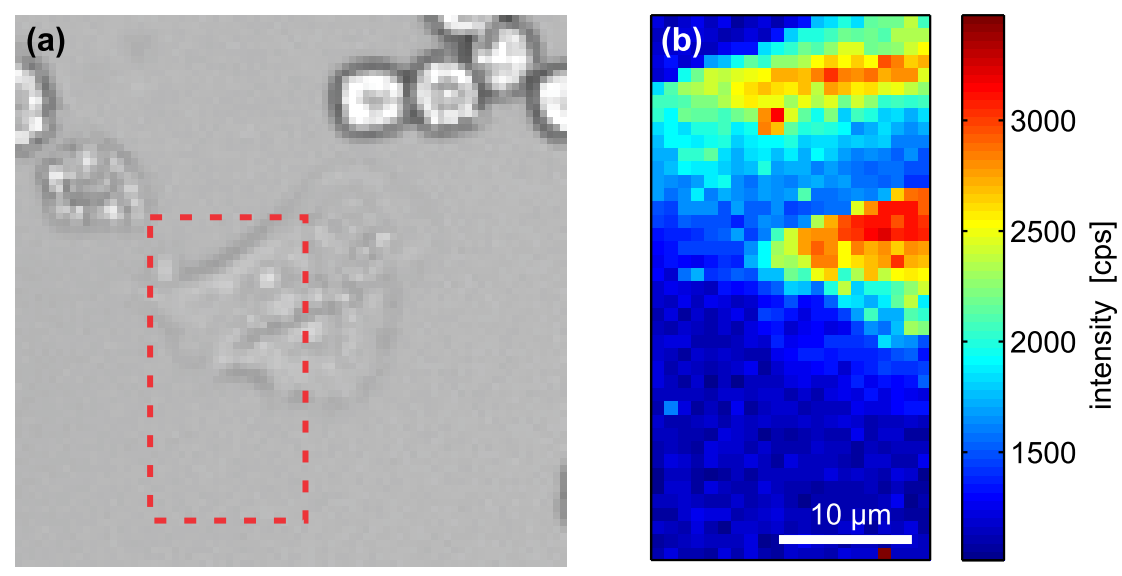

Fig. 7.1.: Bright-field microscopy image and X-ray dark-field image of (initially) living SK8/18-2 cells. (a) Bright-field image of SK8/18-2 cells taken directly before device assembly. (b) X-ray dark-field image taken on the marked region in panel (a). (Live Cells II.4, position 7; ID13, Nov. 2012) 
The living cells can be identified in the dark-field image, but it is not possible to clearly identify the nucleus or smaller substructure in the cell. Due to the long total scan time of about 25 min for the scan shown here on a small area, the cells were detached from the substrate after this measurement and finer measurements or a measurement on the right half of the cells were not possible. Here, a fast (continuous) scanning mode could help to image the living cells before they actively react to the radiation (damage) and detach from the substrate. 


\subsection{Results from Measurements at the P10 Beamline}

In the following section, an example for measurements and results on living cells at the P10 beamline is presented. X-ray dark-field images as well as average radial intensity profiles and the corresponding fits with power laws from an additional measurement are documented in the supplementary material in sectionD.1. An overview of the presented measurements on fixed-hydrated cells taken at the P10 beamline along with the employed scan parameters and the estimated average radiation doses per step is shown in Tab.7.2.

\begin{tabular}{lllllll}
\hline sample & set-up & $\Delta_{y, z}\left[\mu \mathrm{m}^{2}\right]$ & $N_{y} \times N_{z}$ & $T[\mathrm{~s}]$ & $D[\mathrm{~Gy}]$ \\
\hline Live Cells II.7, pos. 7 & March 2013 & $1 \times$ & $\times$ & $51 \times 23$ & 0.05 & $1.8 \times 10^{6}$ \\
Live Cells II.7, pos. 8 & March 2013 & $1 \times$ & 3 & $61 \times 51$ & 0.05 & $2.4 \times 10^{6}$ \\
\hline
\end{tabular}

Tab. 7.2.: Summary of the scan parameters and the estimated average radiation doses per step for the measurements on living cells at the P10 beamline. Here, $\Delta_{y, z}$ are the lateral step sizes, $N_{y}$ and $N_{z}$ the number of scan points in $y$ - and $z$-direction, $T$ is the exposure time and $D$ the estimated average radiation dose per step. All measurements were performed without attenuators in the beam path.

\subsubsection{X-Ray Dark-Field Images}

A bright-field microscopy image on a part of one sample of living SK8/18-2 cells is displayed in Fig. 7.2 a. The bright-field image was taken directly before the assembly of the microfluidic device. The device was aligned at the set-up using the onaxis beamline microscope and images of the cells were taken before and after the measurement. Fig 7.2 b shows an image taken with the beamline microscope on the same sample region as in panel a. The cells appear differently in the beamline microscope image, since this microscope is operating in reflection geometry, meaning that the illumination and the objective are on the same side of the sample. However, the over-all cell shape is similar, which confirms that the cells are alive at the start of the measurement. For the measurement, the microscope was moved out of the beam path, in order to not (partially) block the scattering signal. The X-ray dark-field image recorded on the marked sample region in panel a and $\mathrm{b}$ is displayed in Fig. 7.2 . For the calculation of the X-ray dark-field image, the same detector mask as for the fixed-hydrated cells was used (compare Fig. 6.14).

The two cells in the center of the bright-field microscopy image, can be identified in the X-ray dark-field image. The nuclei can be distinguished from the cell body by 
(a)

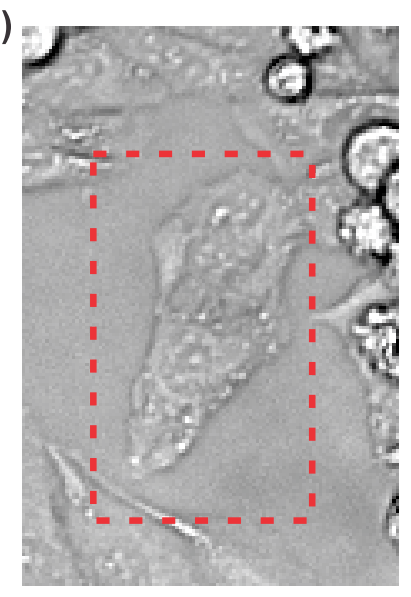

(b)

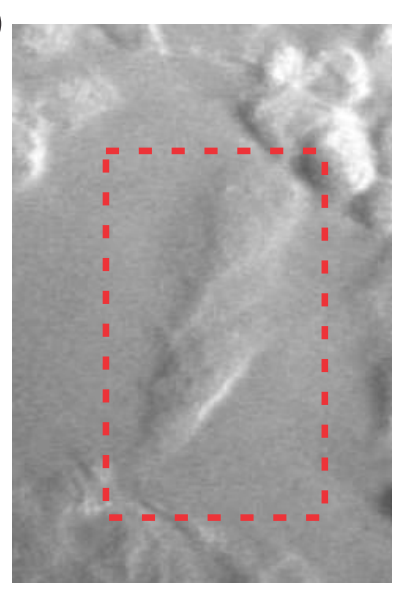

(c)

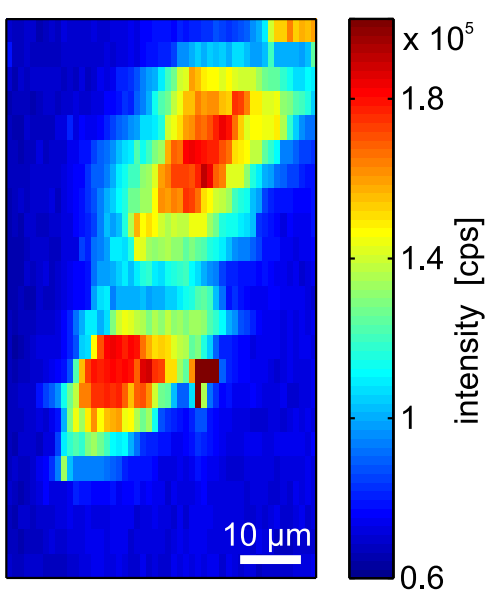

Fig. 7.2.: Visible light microscopy images and X-ray dark-field image of living SK8/182 cells. (a) Bright-field microscopy image of SK8/18-2 cells taken directly before device assembly. (b) Image taken with the beamline microscopy directly before the measurement. (c) X-ray dark-field image taken on the marked region in panel (a) and (b). (Live Cells II.7, position 7; P10, March 2013)

a stronger scattering signal. Due to the larger step sizes for the measurements on living cells as compared to the fixed-hydrated samples, no further internal cellular structures in the nuclei or in the cell bodies can be identified.

\subsubsection{Radial Intensities of Averaged Scattering Patterns}

In analogy to the analyses of the fixed-hydrated cells, three different scan regions were selected, i.e. the nucleus, the cell body and an empty region as shown in Fig. 7.3 . As also discussed for the measurements on the fixed-hydrated cells, the empty region was chosen such that it was located roughly in the same scan lines where the cell was located in order to avoid variations in the background signal due to drifts in the set-up during the measurement. The scattering patterns recorded on the three different regions were averaged and the averaged scattering patterns are shown in Fig. $7.3 \mathrm{~b}-\mathrm{d}$. As also discussed in the previous chapters, the streak-like scattering in the averaged scattering patterns from the empty region can be attributed to the tails of the KB beam and to scattering from the apertures. In the averaged scattering patterns from the nucleus and the cell body, scattering from the cells can be identified.

The averaged scattering patterns were integrated in azimuthal direction, yielding in the radial intensity profiles shown in Fig. 7.4 a. Both radial intensity profiles from the cell are well above the background signal and the scattering signal from the nucleus is stronger than the scattering from the cell body. Subtraction of the background 
(a)

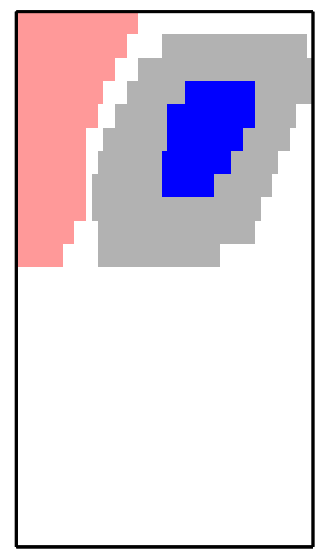

(c)

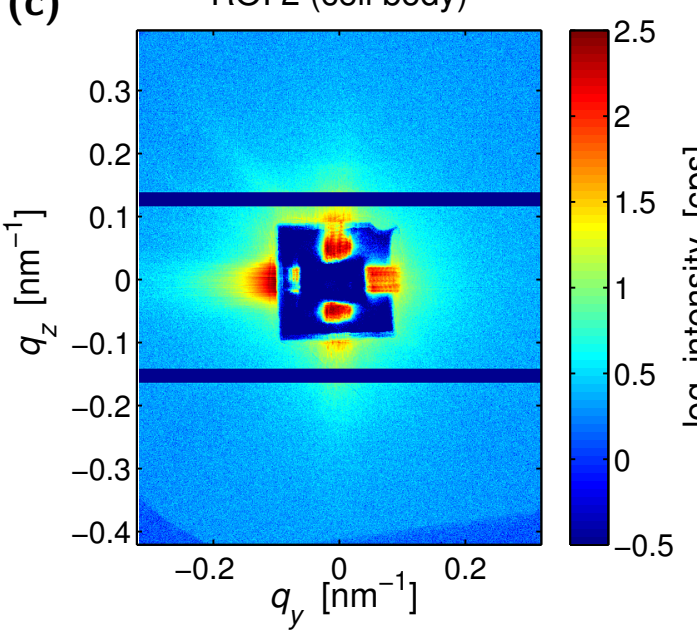

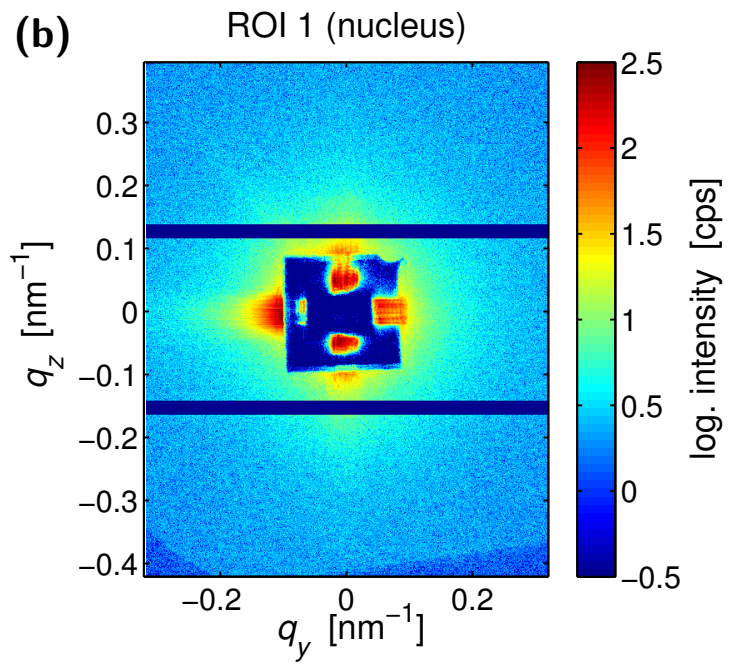

(d)

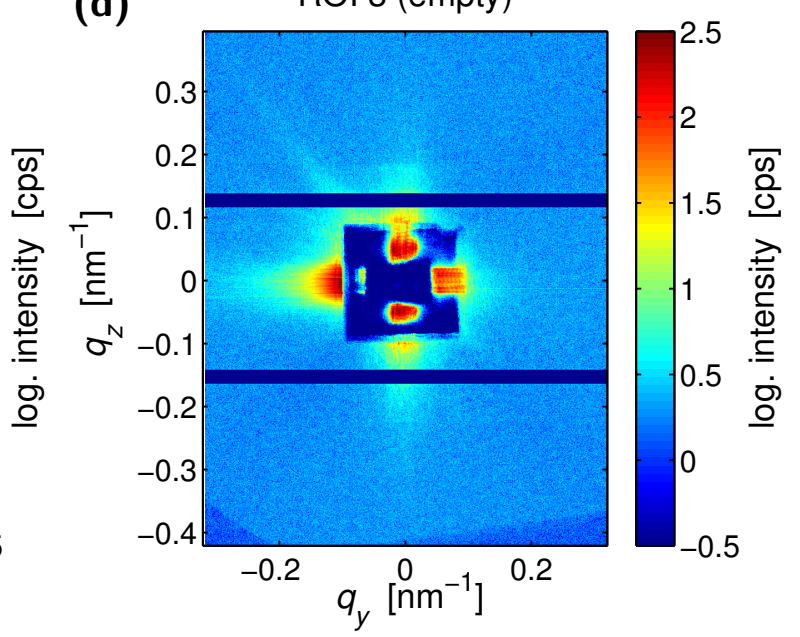

Fig. 7.3.: Averaged scattering patterns from three different scan regions. (a) Selection of three different scan regions, i.e. the nucleus (blue, ROI 1), the cell body (gray, ROI 2) and an empty region (red, ROI 3). (b) Averaged scattering pattern from the nucleus, (b) the cell body and (c) the empty region. (Live Cells II.7, position 7; P10, March 2013)

signal results in the curves shown in Fig. $7.4 \mathrm{~b}$. In contrast to the analyses of the fixed-hydrated and the freeze-dried cells, the background corrected radial intensity profile of the (initially) living cells do not display a concave shape, which indicates a difference in the cellular structure between these sample types. Therefore the intensity decay from the living cells can be described by one power law function over the full $q_{r}$-range. Fits of the background corrected radial intensity profiles with power law functions are shown Fig. 7.5 along with the data points. The $q_{r}$-ranges over which the fit functions are plotted corresponds to the regions that were used for fitting.

The same analysis scheme was applied to in total eight living cells that were mea- 
(a)

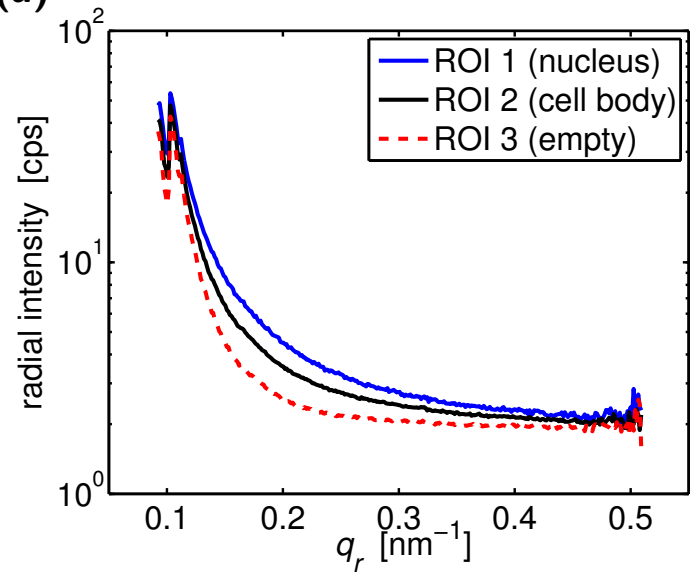

(b)

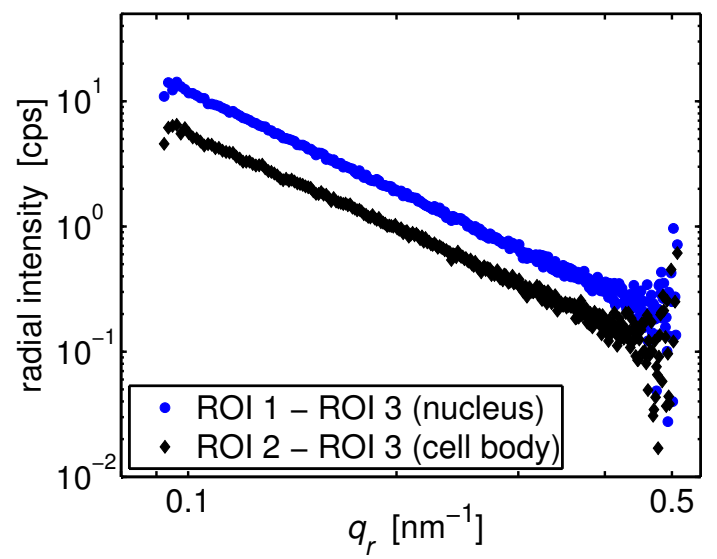

Fig. 7.4.: Radial intensity profiles of averaged scattering patterns. (a) Radial intensity profiles from the nuclear region, the cell body and the empty region. (b) Background corrected radial intensity profiles from the nuclear region and the cell body. (Live Cells II.7, position 7; P10, March 2013)

sured at the P10 beamline in March 2013. All radial intensity profiles along with the power law fits and maps of the selected scan regions for averaging of the scattering patterns are documented in the supplementary information in section D.1. Fig. 7.6 presents the power law exponents for all living cells obtained for the nuclear region and the cell body. All power law exponents lie in a range of -2.5 to -3.0 . The power law exponents obtained on the cell body vary more than the exponents on the nucleus, which can be attributed to the lower scattering signal from the cell body. Mean power law exponents for living cells were determined by averaging all power

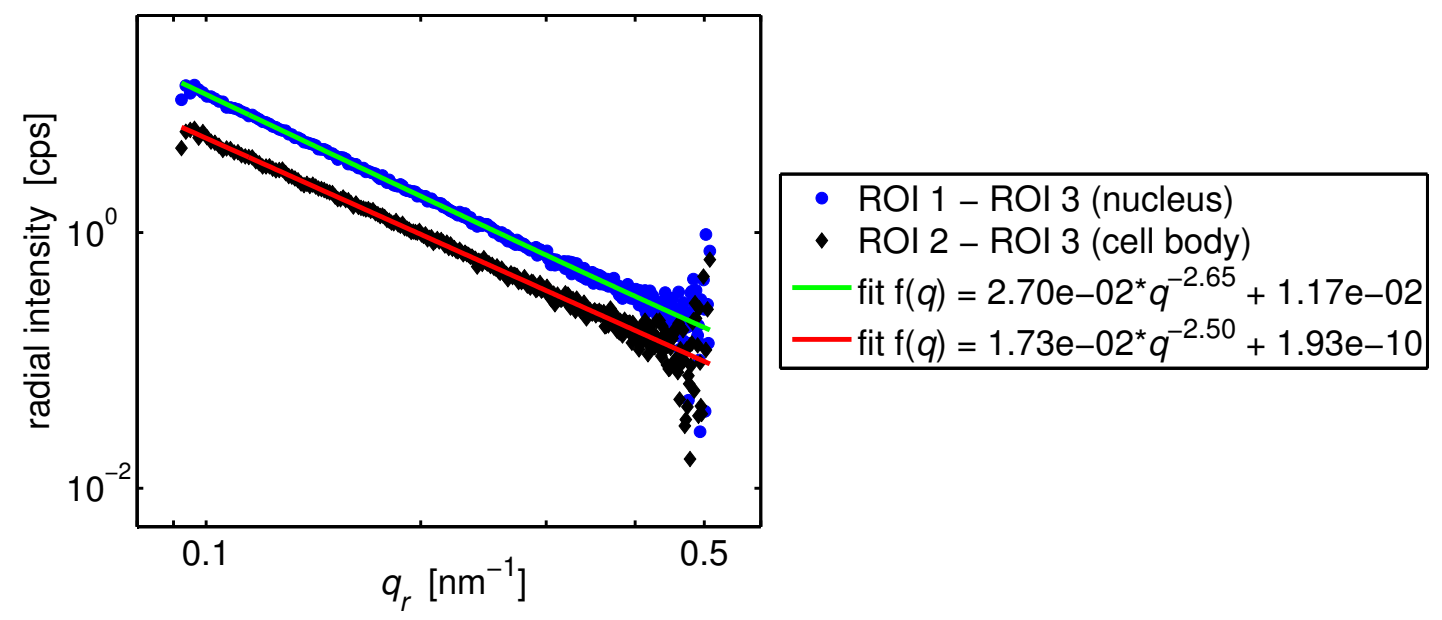

Fig. 7.5.: Fitting of a power law to the background corrected average radial intensity profiles. The $q_{r}$-ranges over which the fit functions are plotted corresponds to the regions that were used for fitting. (Live Cells II.7, position 7; P10, March 2013) 


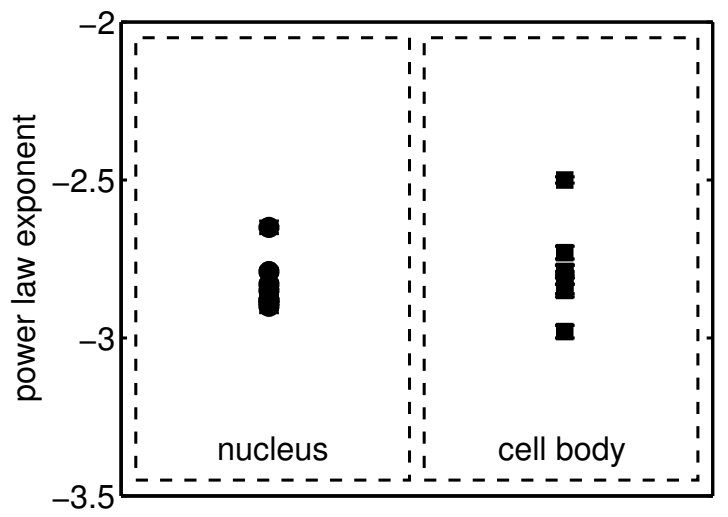

Fig. 7.6.: Comparison of the power law exponents for living cells. The error bars indicate the errors obtained from the fits with a power law. All measurements shown in this figure were performed without attenuators. (P10, March 2013)

law exponents taking into account the position on the cell, i.e. the nucleus or the cell body. The mean power law exponents and the standard deviation of the mean are presented in Tab. 7.3. Again, for averaging the "normal" average and not the "weighted" average with regard to the error was used, because the errors bars only include the goodness of the fits and no information about the accuracy measurement or the state of the measured cell. The power law exponents from the two different regions of the cell agree within the error range. Therefore, it is not possible to distinguish between the nuclear region and the cell body from the intensity decay of the averaged scattering patterns.

\begin{tabular}{lc}
\hline & mean power law exponent \\
\hline nucleus & $-2.83 \pm 0.03$ \\
cell body & $-2.79 \pm 0.05$ \\
\hline
\end{tabular}

Tab. 7.3.: Mean power law exponents and standard deviation of the mean for all (initially) living SK8/18-2 cells measured at the P10 beamline. (P10, March 2013) 


\subsection{Specific Challenges}

For the measurements on living cells, the same experimental problems as described for fixed-hydrated cells in section 6.5 need to be considered. Further challenges for the measurements and the sample preparation arise from the fact that the cells are living and that thy therefore continuously proliferate and change their morphology.

\subsubsection{Storage and Culture of Living Cells}

For all living samples, the cells were grown on $\mathrm{Si}_{3} \mathrm{~N}_{4}$ membrane windows in Göttingen and transported to the synchrotron facilities in microtubes filled with the normal culture medium, which we commonly employ for the cell culture (DMEM with $10 \%$ FCS and Pen-Strep). The cells survived in the sealed tubes for about 2-4 days at room temperature allowing for storage at the beamline without further equipment. Therefore, the equipment needed for handling these samples on-site can be reduced to a minimum and in particular no cell culture lab is needed. However, keeping the living cells in an incubator at normal growth conditions and assembling the microfluidic devices in a clean bench, as it was possible for the measurements at the P10 beamline, is ideal since well-defined conditions for cells growth and sample preparation are obtained.

Due to the continuous proliferation of the cells and linked to that the confluent coverage of the $\mathrm{Si}_{3} \mathrm{~N}_{4}$ membrane with cells, on-site cell culture is necessary for beamtimes longer than about three days.

\subsubsection{Imaging of Living Cells}

For all measurements precise knowledge of the location of the cells on the $\mathrm{Si}_{3} \mathrm{~N}_{4}$ membrane is important. For fixed-hydrated and freeze-dried cells, images of the whole membrane were taken in Göttingen at several steps of the sample preparation. Furthermore, for freeze-dried cells, the cells could be easily identified using the available microscope at set-ups at the beamlines. However, since living cells continuously proliferate or change their morphology, images needed to be recorded directly before the measurements. To this end, microscopes at the synchrotron facilities were employed. However, only bright-field contrast was available and it was not possible to record fluorescence microscopy images of the keratin network in the living cells. The imaging capabilities of the beamline microscopes in the experimental hutches allowed for the correlation of the bright-field microscopy images with the images of the beamline microscopes. However, identifying the cells only with the beamline microscopes (completely without the bright-field microscopy images), would have 
been hardly possible.

\subsubsection{Radiation Damage}

For living cells, radiation damage is even more critical than for fixed-hydrated cells. Living cells can actively react to the X-ray induced damage by, e.g., the start of apoptosis [111. However, for the exposure to radiation doses much lower than the doses applied here, these effects have been shown to occur on time scales of several hours [111], which is long compared to one measurement (well beyond one hour of total scan time). Fig. 7.7 a and b show images of a group of cells taken with the on-axis beamline microscope at the P10 beamline before and after the measurement. The X-ray dark-field image from this scan is depicted in Fig. 7.7k. Similar to the measurements on fixed-hydrated cells, the cells are hardly visible before the measurement and after the measurements the cells appear more whitish in the image, which indicates structural modifications to the cells upon exposure to X-rays. Furthermore, the extension of the left cell in the center of Fig. 7.7a, indicated by an arrow, seems to be thinner in the microscopy image after the measurement and also in the X-ray dark-field image. However, this could be also related to imaging artifacts of the microscope. At the resolution of the beamline microscope it is hard to identify the cell contour before the measurement and therefore the changes upon irradiation are difficult to quantify.
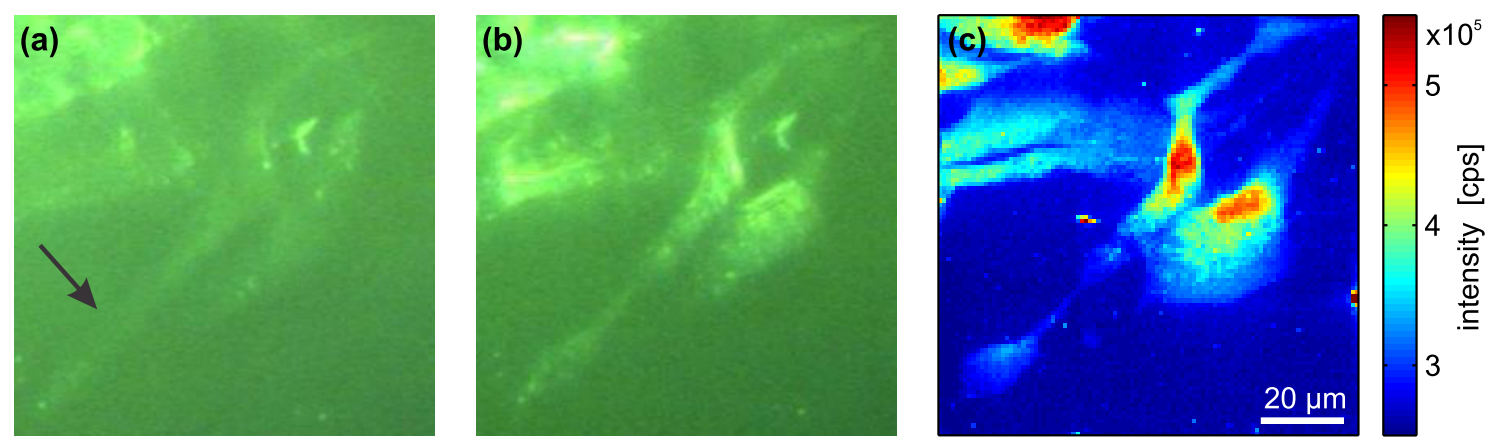

Fig. 7.7.: Radiation damage to living SK8/18-2 cells during a measurement at the P10 beamline. (a) Image taken with the on-axis beamline microscope before and (b) after the measurements. (c) X-ray dark-field image of the cells. (Sample BW235, position 1; P10, March 2013) 


\subsection{Summary and Closing Discussion}

In this chapter, the feasibility of scanning X-ray diffraction experiments on living SK8/18-2 cells was demonstrated. For all measurements on living cells, microfluidic devices were used as a sample environment. Effects of the device material on the cells, e.g. of the two component glue that was used for sealing of the window edges, were not observed. The cells were kept in the devices for several hours and inspection of the cells using the beamline microscopes yielded normal cell shapes for the non-exposed cells. However, for further experiments, the implementation of a temperature control for keeping the cells at $37^{\circ} \mathrm{C}$, i.e. at physiological conditions, would be an improvement. At room temperature, no proliferation of the cells was observed and it can be further expected that the whole metabolism is altered due to different enzyme activities. Therefore also the structural properties of the cells might be influence by the temperature.

Bright-field microscopy images of the cells were recorded directly before device assembly. Unfortunately, we could not record fluorescence microscopy images of the keratin network. However, the lacking of the fluorescence microscopy images was not problematic for the results presented here, since the resolution of the X-ray dark-field imaged was too low to identify structures that could possibly correspond to the keratin network. Furthermore, no difference was obtained for cells with or without keratin for the fixed-hydrated samples and therefore the living cells would probably exhibit no difference either. However, the possibility to record fluorescence microscopy images might become important for future experiments. Furthermore, a calibrated beamline microscope with a higher resolution and the option to use fluorescence microscopy would be ideal to monitor the living cells during and in-between the X-ray measurements. In this context, it would be also interesting to study the effect of or the cell's response to single short exposures with a hard X-ray nano-focused beam at different positions on the same cell, on the substrate next to the cell, or on neighboring cells.

X-ray dark-field images were generated from mesh scans on the living SK8/18-2 cells, allowing for the identification of the cells and the nuclei. Here, performing fast scans with a short total scan time was important to decrease the visible degradation of the cell due to radiation damage during the measurement. Measurements at the P10 beamline yielded best results, due to the available continuous scanning mode and the high photon flux. With the same experimental parameters (step size, exposure time) the continuous scans were faster by roughly a factor of eight. Furthermore, the exposure time at P10 beamline could be reduced by a factor of ten compared to the measurements at ID13 beamline. However, the smaller beam size available at the ID13 beamline might be advantageous for experiments that 
require small probe volumes for the determination of local structural parameters. The resolution of the X-ray dark-field images was with at least one micrometer as determined by the employed step sizes comparatively large. Here, the achievable real space resolution in the X-ray dark-field images seems to be limited by the radiation damage to the sample seems. The main reason for radiation damage seemed to be radicals that were created during the exposures and spread in the buffer solution or in the cell itself. By choosing asymmetric scans with a larger step size in vertical direction (in direction of the slow scan axis), sample regions that were destroyed by radicals created during scanning of the previous lines were skipped (compare section 6.5.1).

When aiming at imaging of living cells with a high resolution in real space, it might be worthwhile to employ and optimize other X-ray imaging techniques like ptychographic, holographic or coherent diffractive imaging for living cells 10, 12, 28, 95, 137]. Furthermore, the potentially lower average radiation dose achieved for measurements using these methods would be beneficial for imaging of living cells and might allow for taking several images of one and the same living cell in its native state. Employing X-rays as a probe provides the unique potential for label-free imaging of living cells a resolution below $100 \mathrm{~nm}$. In combination with microfluidic devices as sample environment, high resolution X-ray imaging would allow for probing the effects of chemicals on living cells. Depending on the necessary radiation dose for imaging at the requested resolution, it might be also possible to monitor global structural changes in one and the same cell upon drug treatment. Furthermore, local structural changes could be probed using the diffraction signal in reciprocal space. However, due to the high photon flux needed to obtain a diffraction signal from cellular structures exhibiting a weak electron density contrast, it might be not possible to record a reliable diffraction signal before and after drug treatment on the same position in a cell. Therefore, for this approach, the diffraction signals from different well-chosen positions (on different cells) needed to be recorded at different stages of the experiment, which would make the comparison more complex. Furthermore, many measurements would be needed to obtain reasonable statistics. The radial intensity of the averaged scattering patterns from living cells can be well described by a single power law over the whole $q_{r}$-range, which is different to the data for the fixed-hydrated and the freeze-dried cells where two power laws were employed. The power law exponents obtained from fits to the radial intensity of averaged scattering patterns, are in range of -2.5 to -3.0 , which is larger than the values for the freeze-dried and the fixed-hydrated cells. These differences between the different methods for sample preparation are discussed in chapter 8 . Since a difference between the power law exponents obtained from the nucleus and the 
cell body is not observed, the structural differences between these two cellular compartments cannot be determined from the intensity decay of averaged scattering patterns. Here, subdivision of the cells areas used for averaging of the scattering patterns, for instance the selection of all scattering patterns that were recorded on (thick) keratin bundles, might be helpful for the determination of characteristic intensity decays. This type of analysis would, however, require a high-resolution fluorescence microscope at the synchrotron beamlines to record images of the keratin network in the living cells. As also discussed for the measurement on fixed-hydrated cells, applying the same analysis to other cell types might help to determine characteristic power law exponents for different cell types or for living cells in general. 


\section{Comparison of the Different Preparation Methods}

In this chapter, results from measurements at the P10 beamline on freeze-dried, fixed-hydrated and living cells are compared. The same analysis scheme could be applied to the measurements on all three sample types, which allows for a direct comparison of the results. Parts of this chapter have been prepared for publication [41].

\subsection{Power Law Exponents}

Averaged scattering patterns were integrated in azimuthal direction and fitted with power law functions as described in the sections 5.3.2, 6.4.2 and 7.3.2. This analysis scheme was applied to six freeze-dried cells, ten fixed-hydrated cells and eight living cells. However, whereas the intensity decay can be well described by a single power law for the living cells, two power laws were fitted to the high and low $q_{r}$-regions for the fixed-hydrated and the freeze-dried cells. All power law exponents obtained from these measurements are presented in Fig. 8.1. Here, the power law exponents for the living cells are shown as blue data points. The power law exponents for the fixed-hydrated and the freeze-dried cells obtained at the low and high $q_{r}$-region are shown as gray and black or bright green and dark green data points, respectively. Different values of the power law exponents are obtained for the three different methods of sample preparation. For the freeze-dried cells both for the nucleus and the cell body the power law exponents obtained in the low $q_{r}$-region range from about -3.0 to -3.7 and the exponents obtained in the high $q_{r}$-region range from about -4.3 to -4.6 . For the fixed-hydrated cells the power law exponents obtained in the low $q_{r}$-region range from about -3.0 to -3.5 and the exponents obtained in the high $q_{r}$-region range from about -3.5 to -4.0 . Furthermore, the power law exponents obtained for the cell body are slightly higher than the ones obtained for the nucleus. For the living cells the power law exponents from the nucleus and the cell body range from about -2.5 to -3 .0. For both regions of the cell, the power law exponents obtained for the living cells are higher than the power law exponents for 
the freeze-dried or fixed-hydrated cells in addition, the power law exponents obtained for the fixed-hydrated cells in the high $q_{r}$-region are higher than the corresponding values for the freeze-dried cells.

\subsection{Average Radial Intensity Profiles}

To compare the radial intensity profiles of living, fixed-hydrated and freeze-dried cells, the background corrected radial intensity profiles for the nuclear region and the cell body of each sample type (fixed-hydrated or living) were averaged, yielding the profiles shown in Fig. 8.2. For the measurements on fixed-hydrated cells, only six profiles were included in the average, since these profiles were recorded with the same experimental parameters, i.e. at the same beamtime, as the measurements on all living cells. Only one freeze-dried cell was measured at the beamtime at the P10 beamline in March 2013 without attenuators in the beam path (compare Tab. 5.2) and therefore no averaged radial intensity profile could be determined and instead the radial intensity profile from one measurement is shown.

Interestingly, the living cells scatter stronger at higher $q_{r}$-values as compared to the fixed-hydrated cells and would therefore provide a higher resolution in imaging experiments. At small $q_{r}$-values, the fixed-hydrated cells show slightly stronger scattering signal than the living cells. The scattering intensity from the freeze-dried cell is much stronger than from the hydrated cells over the whole recorded $q_{r}$-range, which can be explained by the higher electron density contrast between the cellular material and the surrounding medium for freeze-dried cells $\left(\Delta \rho_{\text {dry }}=0.4 \mathrm{e}^{-} / \AA^{3}\right)$

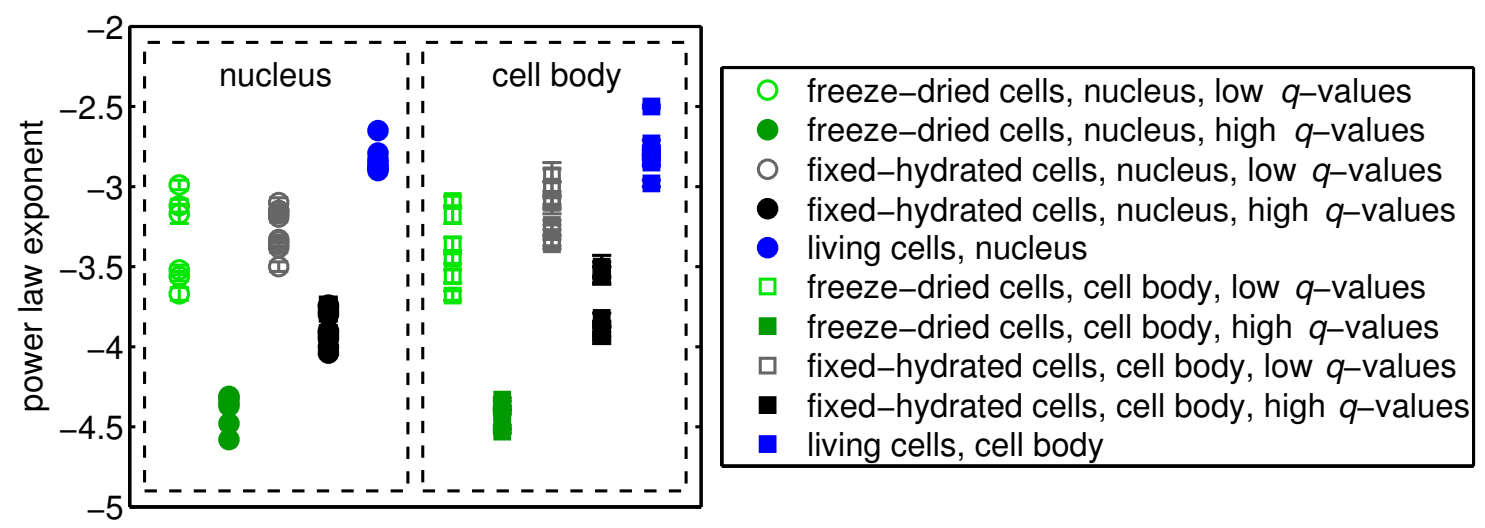

Fig. 8.1.: Comparison of power law exponents obtained from fits to the background corrected radial intensity profiles for freeze-dried, fixed-hydrated and living SK8/18-2 cells. The error bars indicate the errors obtained from the fits with power law functions. 


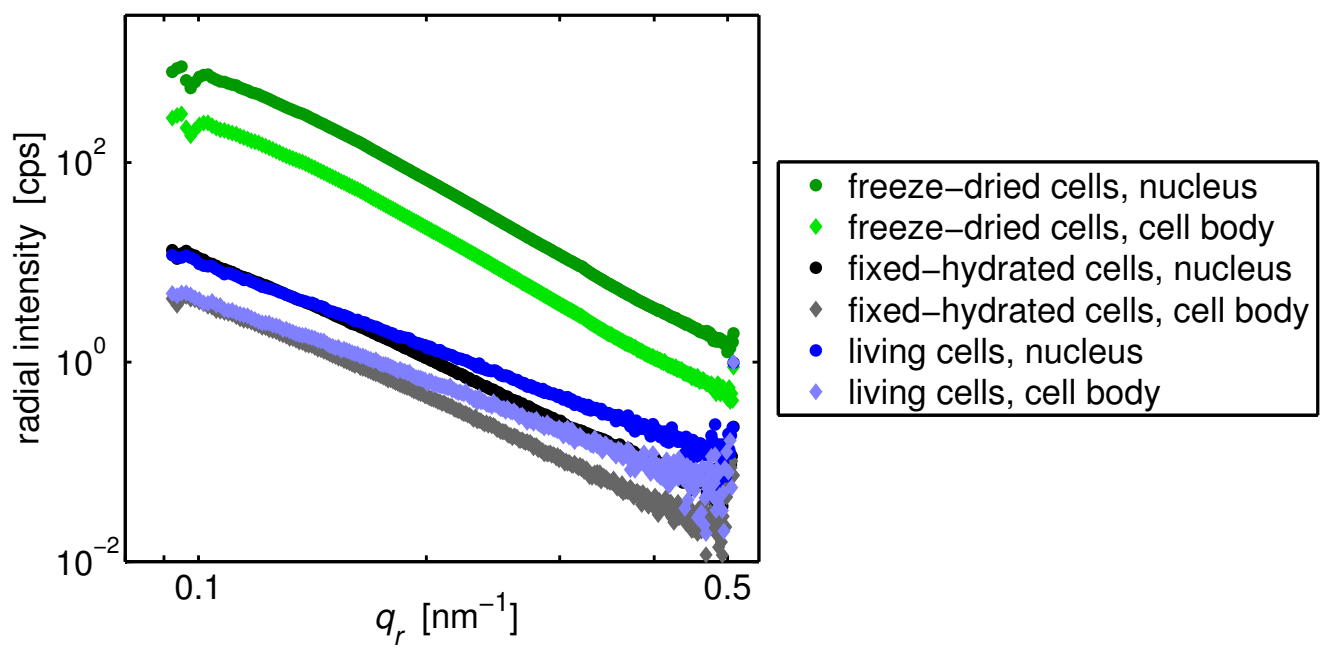

Fig. 8.2.: Averaged radial intensity profiles for eight living cells, six fixed-hydrated cells and one freeze-dried cells for the nucleus and the cell body after background correction.

than for hydrated cells $\left(\Delta \rho_{\text {hyd }}=0.1 \mathrm{e}^{-} / \AA^{3}\right)$.

The average radial intensity profiles for fixed cells were then subtracted from the corresponding profiles for living cells, resulting in the curves presented in Fig. 8.3 ("living cells - fixed-hydrated cells"). Here, the difference curves are plotted against the $q_{r}$-value on a linear scale (bottom axis) and against the corresponding real space sizes (top axis). In the difference plot, positive values denote length scales which are more pronounced in the living cells, whereas negative values represents structures that emerge upon fixation. The difference curves from the nucleus and from the cell body each show a maximum, which is located at around $0.18 \mathrm{~nm}^{-1}$ and $0.14 \mathrm{~nm}^{-1}$

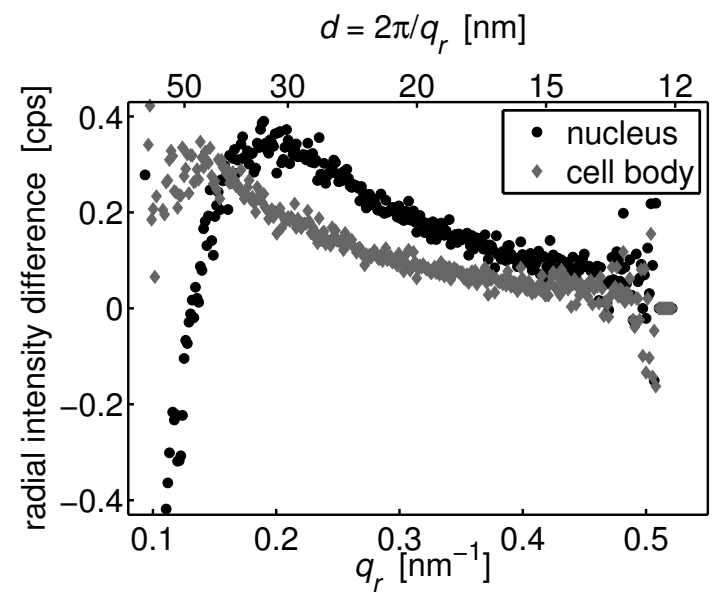

Fig. 8.3.: Difference of the averaged radial intensity profiles for living and fixed-hydrated cells ("living cells - fixed-hydrated cells"). 
corresponding to real space structural sizes of $35 \mathrm{~nm}$ and $45 \mathrm{~nm}$, respectively, meaning that structures in these length scales were destroyed or at least become less ordered upon fixation. Towards high $q_{r}$-values, the difference curves decay to zero. Towards small $q_{r}$-values, the average scattering signal from the fixed-hydrated cells is higher than the signal from the living cells, i.e. the difference curves become negative. This can be interpreted as the formation of aggregates with sizes larger than about $50 \mathrm{~nm}$ or $70 \mathrm{~nm}$ for the nucleus and the cell body, respectively, as a consequence of the chemical fixation with formaldehyde. The crossing point of the averaged radial intensity profiles can clearly be observed for the cell nucleus, and for the cell body the data can be interpolated.

Due to the large difference in signal strength between hydrated and freeze-dried cells, i.e. about two orders of magnitude, difference curves as calculated above for the freeze-dried cells do not contribute additional information about the structural differences between the freeze-dried and hydrated cells, and are therefore not presented here. However, taking into account the logarithmic intensity scaling in Fig. 8.2, the largest difference in the radial intensity profiles can be identified at small $q_{r}$-values, indicating a further increase of the structure sizes in the cells upon plunge-freezing and freeze-drying.

\subsection{Summary and Closing Discussion}

The results from X-ray scattering studies presented here show structural differences between living, fixed-hydrated and freeze-dried cells. The differences between living and fixed-hydrated cells can be explained by the effect of the cell fixation with formaldehyde, which leads to a cross-linking of the proteins in the cells and therefore a size increase of protein aggregates while the amount of small proteins decreases 152$]$. This would result in an increase in scattering signal at small $q_{r}$-values and a decrease at high $q_{r}$-values, which is in line with the above presented observation. A precise understanding and in-depth interpretation of the radial intensity profiles from the living cells and the fixed-hydrated cells, and particularly of the differences between the two systems, requires a detailed and complete model of the nano-scale architecture of living cells and fixed-hydrated cells, which is, however, not available due to the high degree of complexity. To this end, in vitro studies on the effect of formaldehyde on a solution of (a small number of different) proteins and in particular the obtained bulk scattering signals before and after addition of formaldehyde might advance the understanding of the radial intensity profiles from the cells.

The structural differences between living and fixed-hydrated cells reach up to length 
scales of about $65 \mathrm{~nm}$, while the detection of larger length scales was limited by the employed experimental set-up. These length scales are also accessible by different visible light microscopy techniques like stimulated emission depletion (STED) [153, 154]. Here, contrary to our approach, specific cellular components are labeled with a fluorescent dye and only these components are visualized. Given the results presented here, the structural alterations upon cell fixation may potentially become apparent in such experiments and might even influence the result or conclusion as compared to living cells. In this context, studying the effect of other commonly employed fixatives like methanol, glutaraldehyde or completely methanol-free formaldehyde on the cellular structure would be interesting. The usage of microfluidic devices further allows for studying the structural changes during the fixation process, by the addition of formaldehyde to the living cells at the beamline. Furthermore, cell line specific differences in the effect of the (formaldehyde-) fixation should be explored. The largest difference between the radial intensity profiles from the freeze-dried and the hydrated cells, is observed for small $q_{r}$-values, indicating a further increase of the structure size after plunge-freezing and freeze-drying. Different aspects of the preparation process might cause this increase of the structure size. Firstly, too low cooling rates during plunge-freezing might lead to the formation of crystalline ice instead of vitreous ice and the expanding ice crystals might cause local accumulations of cellular material. Secondly, small molecules like proteins, peptides or sugars that are still "dissolved" in the vitreous ice inside the cell upon plunge-freezing might adsorb to larger cellular structures upon the removal of water during freeze-drying. This effect would lead to a coating and linked to that a size increase of the cellular components as observed in the radial intensity profiles.

For the living, fixed-hydrated and freeze-dried samples, different power law exponents $\alpha$ are obtained for the decay $I \propto q^{\alpha}$ of the radial intensity, as displayed in Fig. 8.1. In classical SAXS experiments on bulk samples, i.e. particles in solution, the power law exponent describing the intensity decay in the Porod regime $(q R \gg 1$ with the characteristic particle size $R$, but with the $q$ value small enough to not being sensitive to the atomic spacing [86]) can be attributed to specific structural properties, which will be briefly summarized here. Firstly, the shape of particles in solution gives rise to specific Porod exponents. For instance, a solution of spherical particles yields an Porod exponent of $\alpha=-4$, a solution of disc-like particles yields $\alpha=-2$ and a solution of rod-like particles yields $\alpha=-1[86]$. Secondly, interfacial and fractal properties of the probed system can be studied. In this context, compact two phase systems with sharp interfaces are known to exhibit a power law decay with an exponent of -4 [142, 143], which is changed towards more positive values in the presence of surface roughness. Power law exponents in the range from -1 to 
-4 have been further related to systems exhibiting fractal properties 155 161. In this context, a mass fractal with a fractal dimension $D_{m}$ yields an intensity decay

$$
I \propto q^{-D_{m}}, \text { with } 1 \leq D_{m} \leq 3
$$

and surface fractal with a fractal dimension $D_{s}$ yields an intensity decay

$$
I \propto q^{-\left(6-D_{s}\right)}, \text { with } 2 \leq D_{s} \leq 3
$$

See for example reference [161] for a demonstrative explanation of mass and surface fractals. A key feature of fractals is the invariance of the structure for the observation on different scale (self-similarity over different length scales). Due to this property, a constant power law exponent should be observed over at least one order of magnitude to assure a reasonable analysis of the fractal properties of a system [158. Thirdly, power law exponents smaller than -4 have been attributed to structures with diffuse boundaries, in which the electron density at the interface varies continuously 144 , 145]. For all of these (theoretical) predictions, monodisperse systems of only one kind of particle, e.g. only spheres or only mass fractals, have been considered. By contrast, our objects of study are highly polydisperse both in size and shape of the individual particles like proteins, and therefore a direct relation between the recorded power law exponent for the intensity decay and the underlying structures is not evident. In particular the scattering signal could also be explained by a superposition of scattering from differently shaped particles of different sizes. Here again, a model of the cellular structures is essential for a precise understanding of the resulting power law exponent in the decay of the radial intensity profiles. 


\section{Summary and Conclusion}

The central objective of this thesis was the application of scanning X-ray diffraction using a nano-focused beam to biological cells in order to probe the structure of cytoskeletal bundles and networks of keratin intermediate filaments, making use of the combination of structural information obtained in real space and in reciprocal space. To this end, a controlled nanometer sized beam has been scanned over the cell, recording diffraction patterns at each scan position. X-ray dark-field and differential phase contrast were used to generate real space images of the samples. Three different sample types were employed in these studies: freeze-dried, fixed-hydrated and living SK8/18-2 cells. The SK8/18-2 cells express fluorescently labeled keratins that assemble in these cells into complex networks, which allows for the correlation of fluorescence microscopy image with the X-ray measurements. The examples of data analysis illustrate the different options of combining real space and reciprocal space information.

As a first experimental step, freeze-dried SK8/18-2 cells were studied as described in chapter 5. The preparation of this sample type was well-established and they were easy to handle during the beamtimes, which made them an ideal starting point for the studies presented here. In these cells, structure sizes in the range of 200-400 nm could be identified in the X-ray dark-field image. The signal anisotropy in individual scattering patterns was used to determine the local structure orientation in the sample. Overlay images of orientation maps and X-ray dark-field images showed a network structure, which is similar to the fluorescence microscopy images of the keratin network in the sample. The analysis of single diffraction patterns using azimuthal integration in different angular segments, revealed maxima and minima in the scattering signal in the radial intensity profiles perpendicular to the local structure direction in the sample. The intensity distribution could be approximated by the form factor of a solid cylinder with a diameter of about $42 \mathrm{~nm}$. However, the internal filament arrangement in keratin bundles could not be resolved. Scattering patterns recorded with a larger beam or the average of adjacent scattering patterns showed no scattering maxima and minima, but a smooth decay of the scattered intensity, which indicates a high heterogeneity of the structural properties in the sample. 
Due to the potentially invasive and structure altering steps during the preparation of freeze-dried samples, the next experimental step was the investigation of fixedhydrated and living cells in solution as presented and discussed in chapter 6 and chapter 7. The choice and the design of an appropriate sample environment was a major challenge for these experiments. Sandwich wet chambers built of two silicon nitride membrane windows were employed for first test experiments. As a more versatile sample environment, a novel type of X-ray compatible microfluidic devices based on the UV-curable glue NOA 81 as a moldable material, $8 \mu \mathrm{m}$ thick Kapton foil, and silicon nitride membrane windows as growth substrate for cells was developed. Both types of sample environments worked well in terms of leakage and positional stability.

For the fixed-hydrated cells, X-ray dark-field images with a step size down to $500 \mathrm{~nm}$ were recorded, which allowed for the identification of substructures in the cell body and in the nucleus. For living cells, the step sizes in the mesh scan and correspondingly the real space resolution in the X-ray dark-field images were chosen larger, which still allowed for an identification of the cells and the nuclei, but not for the identification of internal structures. These results demonstrate the feasibility of imaging hydrated cells using X-ray dark-field contrast. The successful combination of microfluidics and scanning X-ray scattering for studying whole hydrated and particularly living cell paves the way for the application of other techniques based on $\mathrm{X}$-rays as a probe, like X-ray fluorescence analysis or different imaging methods, to living cells.

Radial intensity profiles obtained from average diffraction patterns of the complete scans were determined for freeze-dried, fixed-hydrated and living SK8/18-2 cells, respectively. Distinct differences in the radial intensity profiles on length scales from about $10 \mathrm{~nm}$ to $60 \mathrm{~nm}$ were observed not only between freeze-dried and hydrated cells, but also between fixed-hydrated and living cells. Fits of the radial intensity profiles with power law functions yielded characteristic exponents for samples prepared using the three different preparation methods. Therefore, structural differences between the different sample types and in particular between fixed-hydrated and living cells can be monitored by X-ray scattering.

In conclusion, it was demonstrated that small-angle X-ray scattering as a wellestablished biophysical method for in vitro structure analysis of biomolecular assembly can be generalized in a way which is compatible with cellular structure analysis of freeze-dried cells as well as fixed and living cells in solution. The unique combination of structural information obtained in real space and reciprocal space allowed for probing length scales ranging from micrometers down to a few nanometers. This method therefore provides a bridging technique between, on the one hand, 
various imaging methods that use visible light or X-rays and achieve best resolutions of several tens of nanometers 99 11, 153, 154 and, on the other hand, techniques like electron microscopy or X-ray crystallography that reach atomic resolution. Three different sample types, which were prepared using different methods, were studied and structural differences between the sample types at length scales of several tens of nanometers were determined. The successful application to hydrated and living cells further demonstrates the potential for structural analysis at hardly accessible length scales in native samples. The developed microfluidic devices provide a welldefined sample environment for hydrated cells and potentially allow for chemical manipulation of living cells. 



\section{A. Fluorescence Staining Protocols}

\section{A.1. Protocol for Staining of Filamentous Actin and DNA}

\section{Dyes}

Alexa Fluor 546 Phalloidin was purchased from Invitrogen (Darmstadt, Germany) and a stock solution was prepared by dissolving the dye in methanol at a concentration of approximately $6.6 \mu \mathrm{M}$ (compare manual by Invitrogen [162]). For cell staining a working solution was prepared by diluting $20 \mu \mathrm{l}$ stock solution in $800 \mu \mathrm{l} \mathrm{PBS}$.

4',6-diamino-2-phenylindole (DAPI) was purchased from Sigma-Aldrich and used at a working concentration of $1 \mu \mathrm{g} / \mathrm{ml}$ in PBS.

\section{Staining}

Cells were grown on cover slides for 1-2 days and subsequently fixed by adding $3.7 \%$ formaldehyde solution for $20 \mathrm{~min}$ at room temperature. Afterwards the cells are washed three times with PBS, permeabilized with $0.5 \%$ TritonX-100 (Roth) for $20 \mathrm{~min}$ and again washed three times with PBS. The cover slides were taken out of the PBS and placed on parafilm, which allows to add a small amount of liquid directly on the cover slides. $200 \mu \mathrm{l}$ of the Alexa Fluor 546 Phalloidin working solution were added on each cover slide and incubated for $60 \mathrm{~min}$. Afterwards the cover slides were washed three times with $1 \%$ BSA in PBS. $200 \mu$ l of the DAPI working solution were added and incubated for $5 \mathrm{~min}$. The cells were washed three times with PBS and the cover slides were mounted with $25 \mu$ Prolong Gold Antifade Reagent (Invitrogen) on a microscope slide. During all incubation steps the cover slides were kept in the dark to prevent bleaching of the dyes. 


\section{A.2. Protocol for Staining of Microtubules and DNA}

\section{Antibodies and Dyes}

Anti-Bovine $\alpha$-Tubulin was purchased from Invitrogen and a stock solution with a concentration of $200 \mu \mathrm{g} / \mathrm{ml}$ was prepared in $2 \mathrm{mM} \mathrm{NaN}_{3}$ with $1 \%$ BSA in PBS. A working solution with a concentration $1 \mu \mathrm{g} / \mathrm{ml}$ was obtained by diluting $5 \mu \mathrm{l}$ stock solution in $1 \mathrm{ml} \mathrm{1 \%}$ BSA in PBS.

Alexa Fluor 568 Goat Anti-Mouse IgG Antibody was purchased from Invitrogen with a concentration of $2 \mathrm{mg} / \mathrm{ml}$. A working solution with a concentration $5 \mu \mathrm{g} / \mathrm{ml}$ was obtained by diluting $2.5 \mu \mathrm{l}$ stock solution in $1 \mathrm{ml} 1 \%$ BSA in PBS.

DAPI was used at a working concentration of $1 \mu \mathrm{g} / \mathrm{ml}$ in PBS.

\section{Staining}

Cells were grown on cover slides for 1-2 days and subsequently fixed by adding cold methanol and incubated for $15 \mathrm{~min}$ on ice. Afterwards the cells are washed three times with PBS, permeabilized with $0.5 \%$ TritonX-100 for $20 \mathrm{~min}$ and again washed three times with PBS. The cover slides were incubated for 30 min with $1 \%$ BSA in PBS and subsequently taken out of the PBS and placed on parafilm, which allows to add a small amount of liquid directly on the cover slides. $200 \mu \mathrm{l}$ of the primary antibody (Anti-Bovine $\alpha$-Tubulin) working solution were added on each cover slide and incubated for $12 \mathrm{~h}$. After the incubation with the first antibody the cover slides were washed three times with $1 \%$ BSA in PBS. $200 \mu$ l of the second antibody (Alexa Fluor 568 Goat Anti-Mouse IgG Antibody) working solution was added and incubated for $2 \mathrm{~h}$, followed by three washing steps with PBS. $200 \mu \mathrm{l}$ of the DAPI working solution were added and incubated for $5 \mathrm{~min}$. The cells were again washed three times with PBS and the cover slides were mounted with $25 \mu$ Prolong Gold Antifade Reagent on a microscope slide. During all incubation steps the cover slides were kept in the dark to prevent bleaching of the dyes. 


\section{B. Supplementary Data on Freeze-Dried Cells}

\section{B.1. Results from Measurements at the P10 Beamline}

In this section, additional measurements on freeze-dried SK8/18-2 cells at the P10 beamline are presented. For each measurement the fluorescence and phase contrast microscopy image and the X-ray dark-field images as well as fits of the radial intensity profiles of averaged scattering patterns with power law functions are shown. The scan parameters for these measurements are listed in Tab 5.2 in section 5.3 .

\section{Sample BW130, Position 1}
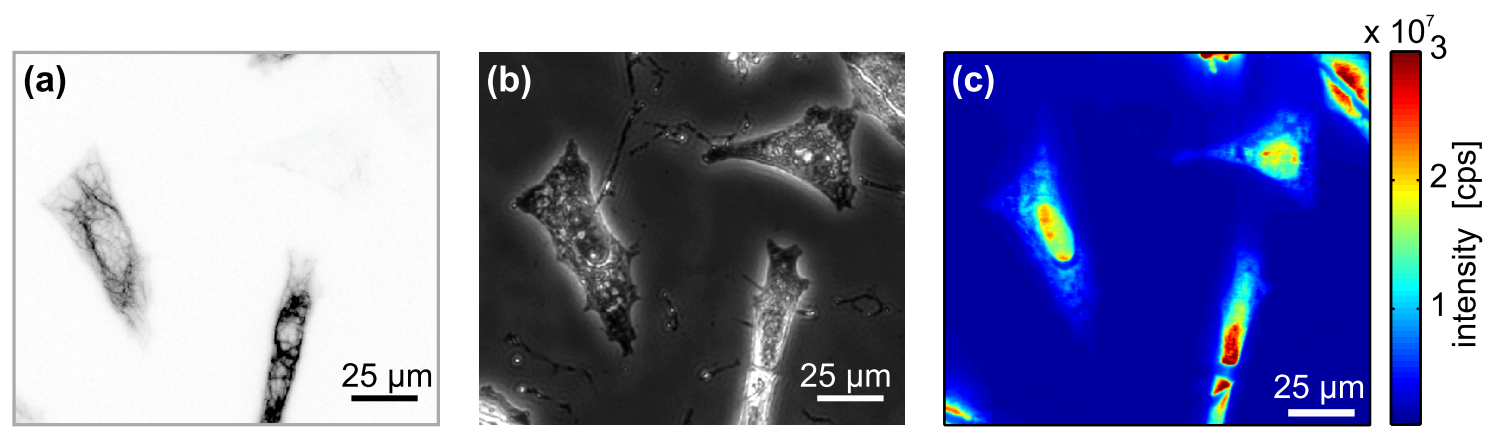

Fig. B.1.: Microscopy images and X-ray dark-field image of a group of SK8/18-2 cells. (a) Fluorescence microscopy image of the keratin network taken before plunge-freezing and freeze-drying and (b) phase contrast image after freeze-drying. The cells on the top-right side of the image does not contain a keratin network. (c) X-ray dark-field image of a scan on the same sample region. (Sample BW130, position 1; P10, Oct. 2012) 


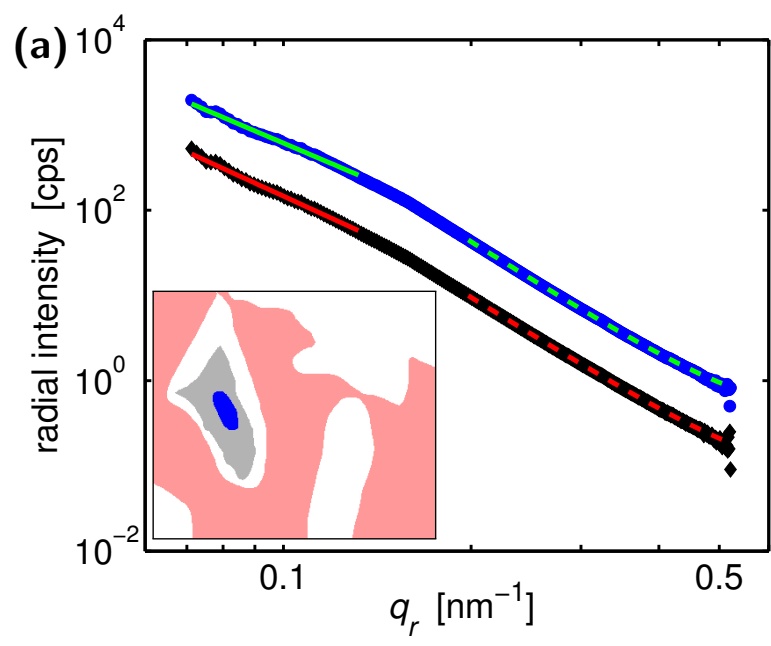

- ROI 1 - ROI 3 (nucleus)

- ROI 2 - ROI 3 (cell body)

- fit $f(q)=4.63 e-01^{*} q^{-3.12}+2.46 e-09$

一 fit $\mathrm{f}(q)=6.42 \mathrm{e}-02^{*} q^{-3.36}+2.11 \mathrm{e}-07$

- - - fit $f(q)=2.68 \mathrm{e}-02^{*} q^{-4.58}+3.16 \mathrm{e}-01$

- - - fit $f(q)=6.72 e-03^{*} q^{-4.50}+5.83 e-02$

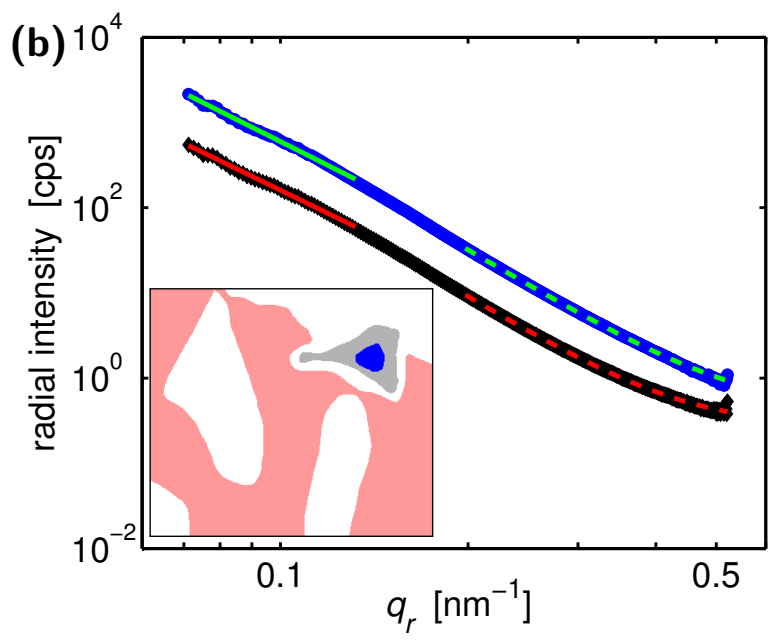

- ROI 1 - ROI 3 (nucleus)

- ROI 2 - ROI 3 (cell body)

- fit $\mathrm{f}(q)=1.27 \mathrm{e}-01^{*} q^{-3.67}+9.68 \mathrm{e}-11$

- fit $\mathrm{f}(q)=4.38 \mathrm{e}-02^{*} q^{-3.56}+1.72 \mathrm{e}-10$

--- fit $f(q)=3.08 \mathrm{e}-02^{*} q^{-4.31}+4.21 \mathrm{e}-01$

- - - fit $f(q)=7.56 e-03^{*} q^{-4.39}+2.71 e-01$

Fig. B.2.: Fitting of the low $q_{r}$-region and high $q_{r}$-region of the background corrected average radial intensity profiles from the nucleus and the cell body with power laws, respectively. The inset shows the regions of the scan that were used for averaging of the scattering patterns (blue: nucleus, ROI 1; gray: cell body, ROI 2; red: empty, ROI 3). The $q_{r}$-ranges over which the fit functions are plotted correspond to the regions that were used for fitting. (Sample BW130, position 1; P10, Oct. 2012) 


\section{Sample BW130, Position 2}
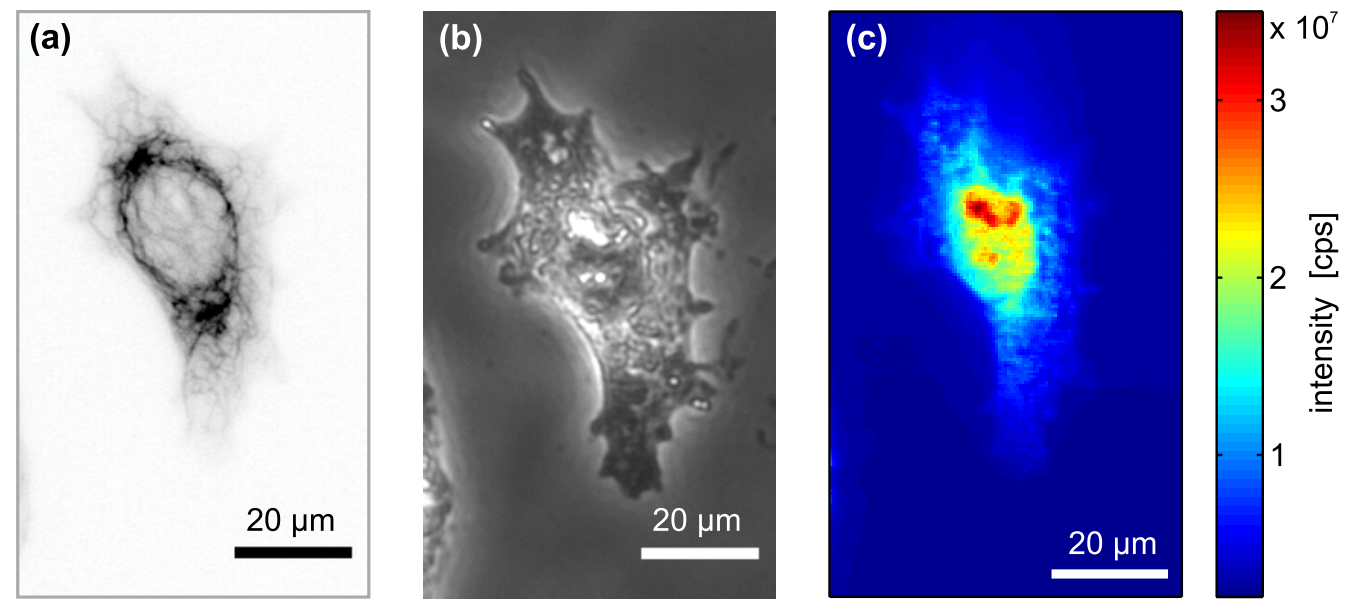

Fig. B.3.: Microscopy images and X-ray dark-field image. (a) Fluorescence microscopy image of the keratin network taken before plunge-freezing and freeze-drying and (b) phase contrast image after freeze-drying. (c) X-ray dark-field image reconstructed from a scan on the same sample region. (Sample BW130, position 2; P10, Oct. 2012)

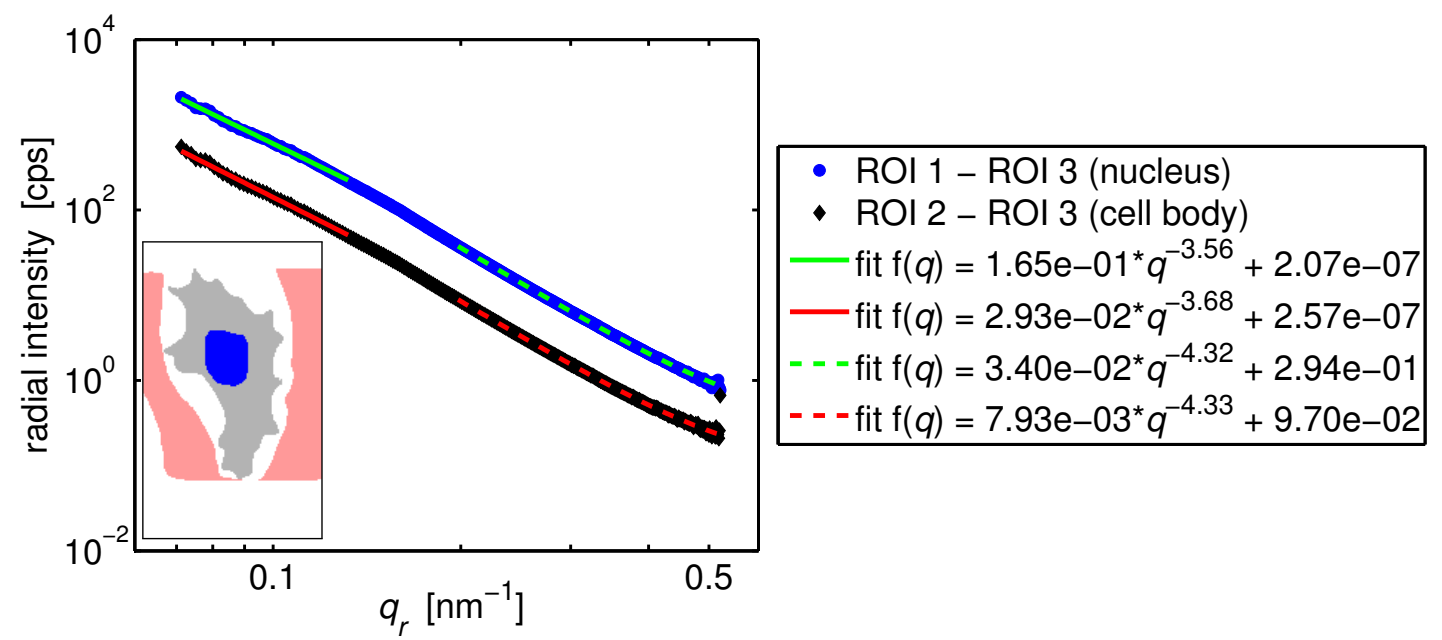

Fig. B.4.: Fitting of the low $q_{r}$-region and high $q_{r}$-region of the background corrected average radial intensity profiles from the nucleus and the cell body with power laws, respectively. The inset shows the regions of the scan that were used for averaging of the scattering patterns (blue: nucleus, ROI 1; gray: cell body, ROI 2; red: empty, ROI 3). The $q_{r}$-ranges over which the fit functions are plotted correspond to the regions that were used for fitting. The data were taken at P10, Oct. 2012. 
(a) $N y=52, N z=55$

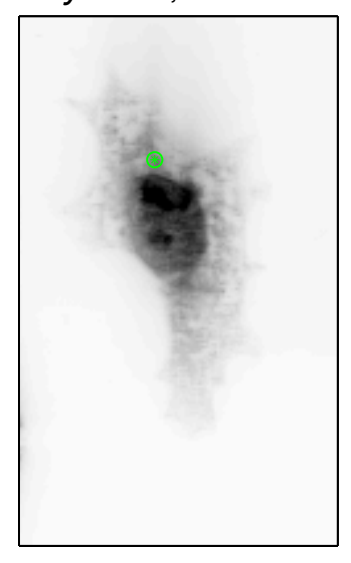

(b) image $\mathrm{Ny}=52, \mathrm{Nz}=55$

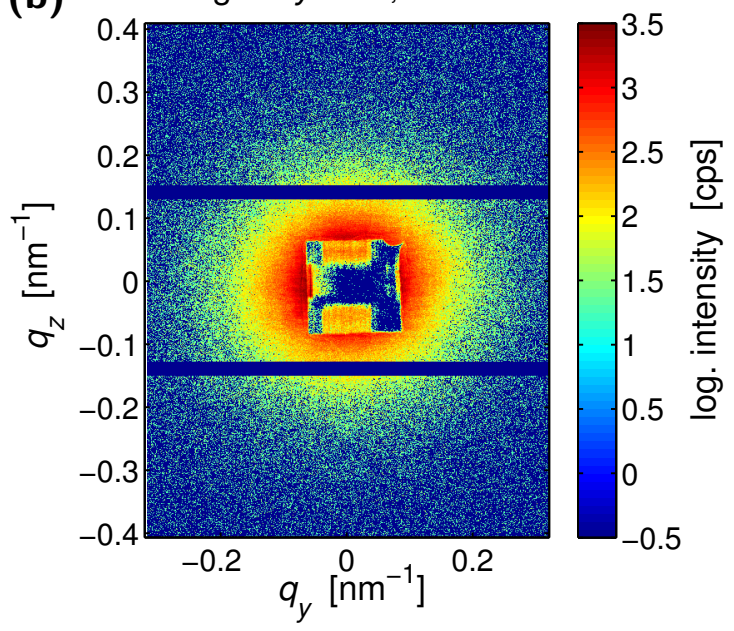

Fig. B.5.: Single scattering pattern from a scan with a step size of $500 \mathrm{~nm}$. (a) Position of the scattering pattern in the scan. (b) Single scattering pattern. (Sample BW130, position 2; P10, Oct. 2012) 


\section{Sample BW201, Position 2}
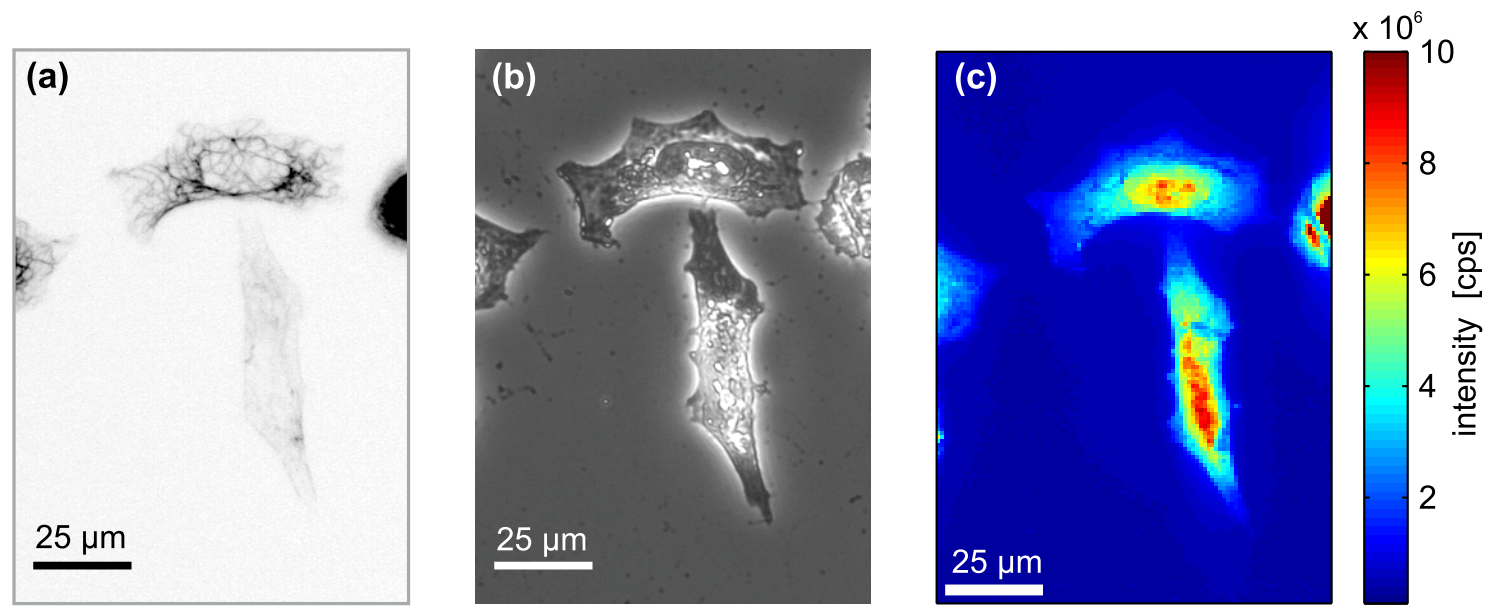

Fig. B.6.: Microscopy images and X-ray dark-field image. (a) Fluorescence microscopy image of the keratin network taken before plunge-freezing and freeze-drying and (b) phase contrast image after freeze-drying. (c) X-ray dark-field image reconstructed from a scan on the same sample region. For better comparability, the dark-field image is normalized on the transmission of one attenuator. For Further analysis only the cell at the top of the scan was used, because the cell at the bottom shown several cracks in the cell body. (Sample BW201, position 2; P10, March 2012)

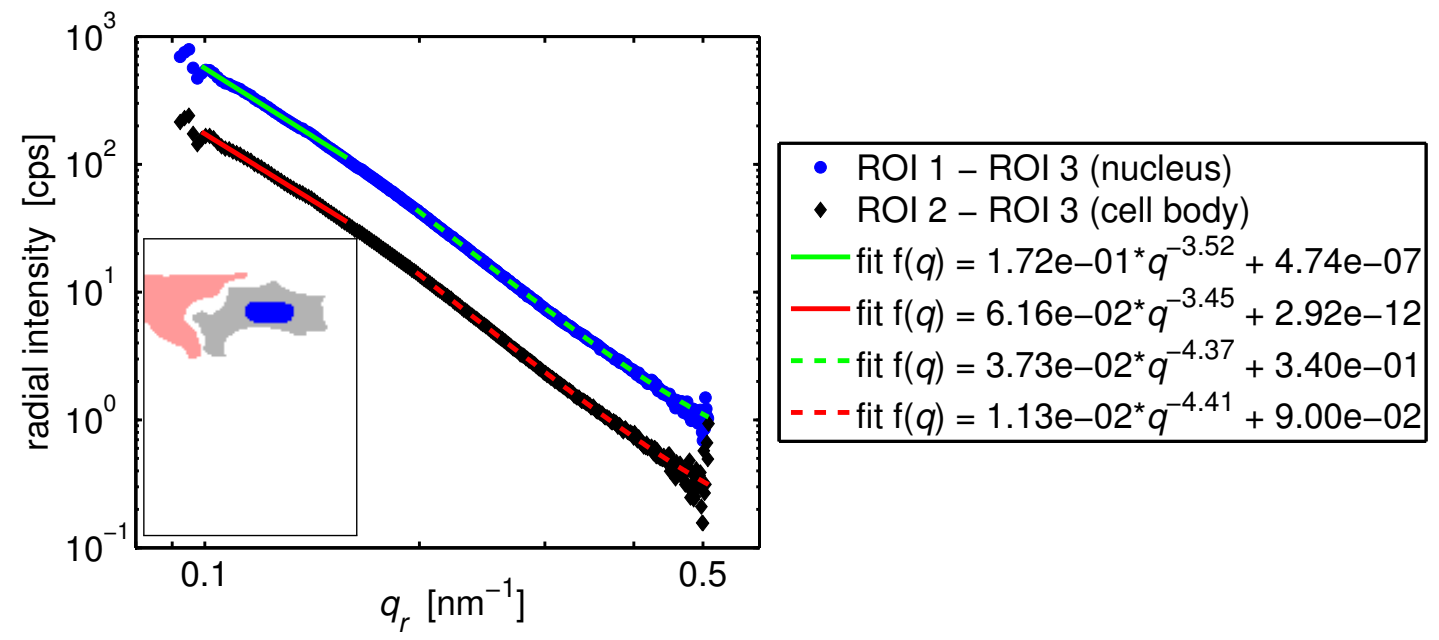

Fig. B.7.: Fitting of the low $q_{r}$-region and high $q_{r}$-region of the background corrected average radial intensity profiles from the nucleus and the cell body with power laws, respectively. The inset shows the regions of the scan that were used for averaging of the scattering patterns (blue: nucleus, ROI 1; gray: cell body, ROI 2; red: empty, ROI $3)$. The $q_{r}$-ranges over which the fit functions are plotted correspond to the regions that were used for fitting. For better comparability, the radial intensity was normalized on the transmission of one attenuator. (Sample BW201, position 2; P10, March 2013) 


\section{Sample BW201, Position 3}
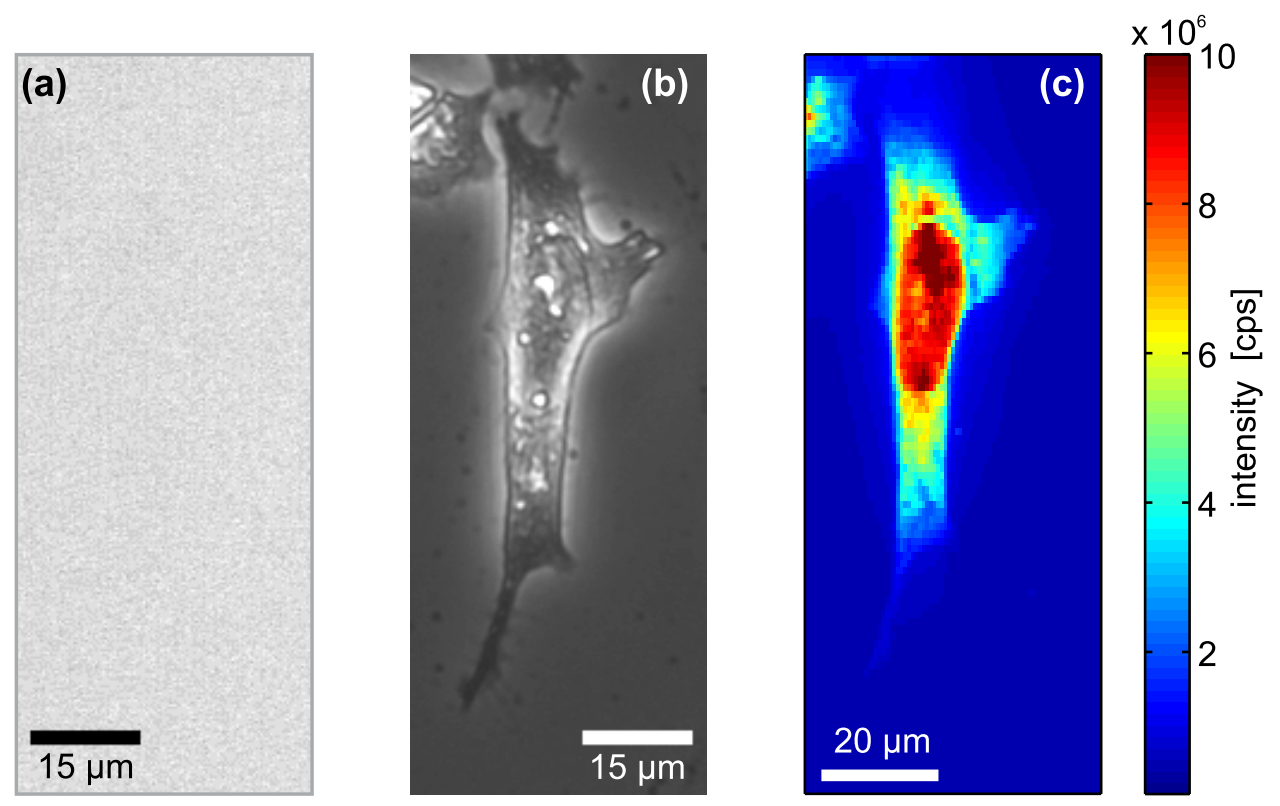

Fig. B.8.: Microscopy images and X-ray dark-field image. (a) Fluorescence microscopy image of the keratin network taken before plunge-freezing and freeze-drying and (b) phase contrast image after freeze-drying. (c) X-ray dark-field image reconstructed from a scan on the same sample region. (Sample BW201, position 3; P10, March 2012)

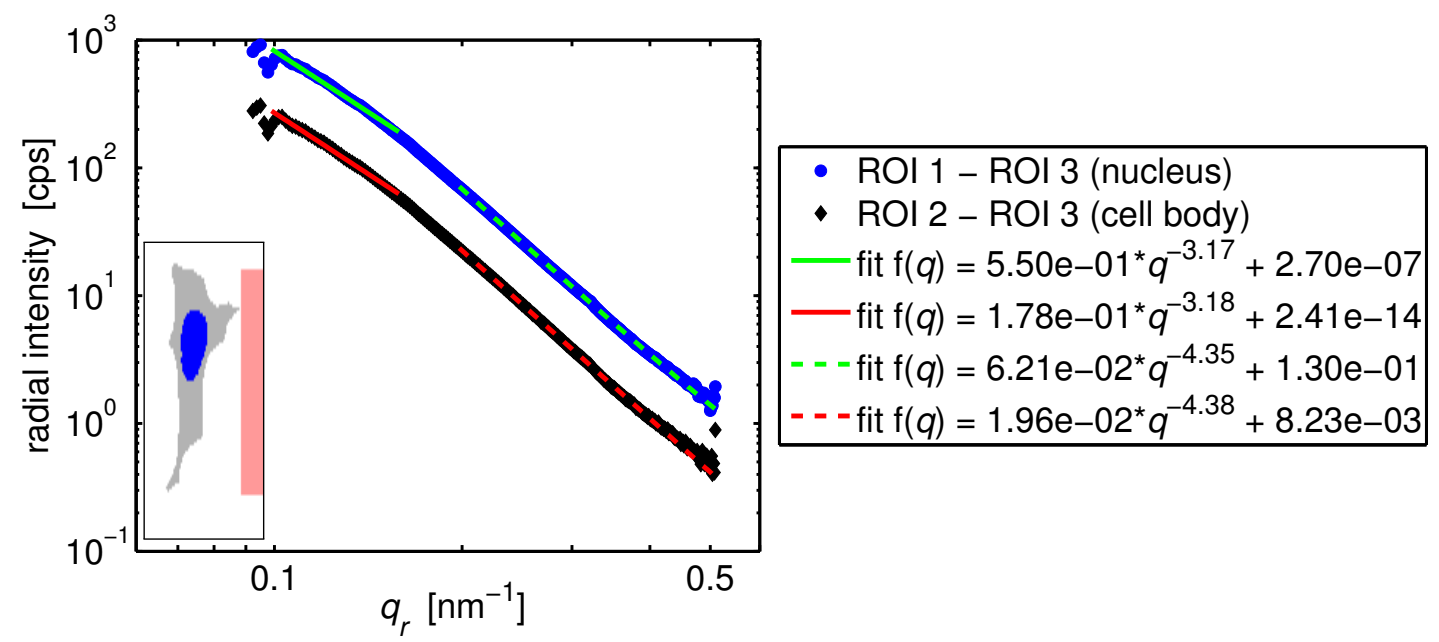

Fig. B.9.: Fitting of the low $q_{r}$-region and high $q_{r}$-region of the background corrected average radial intensity profiles from the nucleus and the cell body with power laws, respectively. The inset shows the regions of the scan that were used for averaging of the scattering patterns (blue: nucleus, ROI 1; gray: cell body, ROI 2; red: empty, ROI 3). The $q_{r}$-ranges over which the fit functions are plotted correspond to the regions that were used for fitting. (Sample BW201, position 3; P10, March 2013) 


\section{Supplementary Data on Fixed-Hydrated Cells}

\section{C.1. Results from Measurements at the ID13 \\ Beamline}

In this section, an additional measurement on fixed-hxdrated SK8/18-2 cells at the ID13 beamline is presented. The fluorescence and phase contrast microscopy image and the X-ray dark-field images as well as fits of the radial intensity profiles of averaged scattering patterns with power law functions are shown. The scan parameters for these measurements are listed in Tab.6.2 in section 6.3.

\section{Sample BW171, Position 1}

(a)

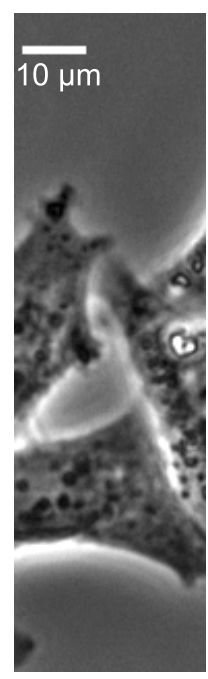

(b)

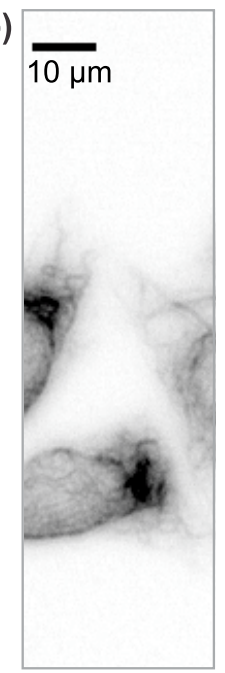

(c)

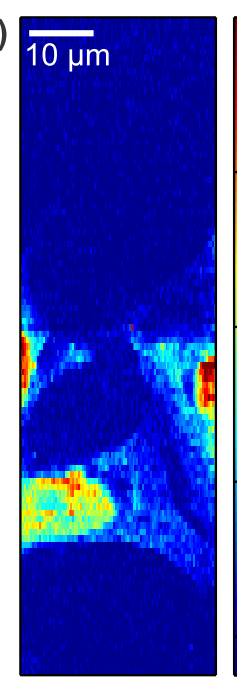

2400

2000

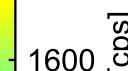

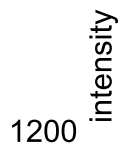

800

Fig. C.1.: Microscopy images and X-ray dark-field image of a fixed-hydrated SK8/18-2 cell. (a) Visible light phase contrast microscopy image and (b) fluorescence microscopy image of the keratin network in SK8/18-2 cells that were fixed with formaldehyde. (c) X-ray dark-field image of the same sample region as in (a) and (b). (Sample BW171, position 1; ID13, Nov. 2012) 


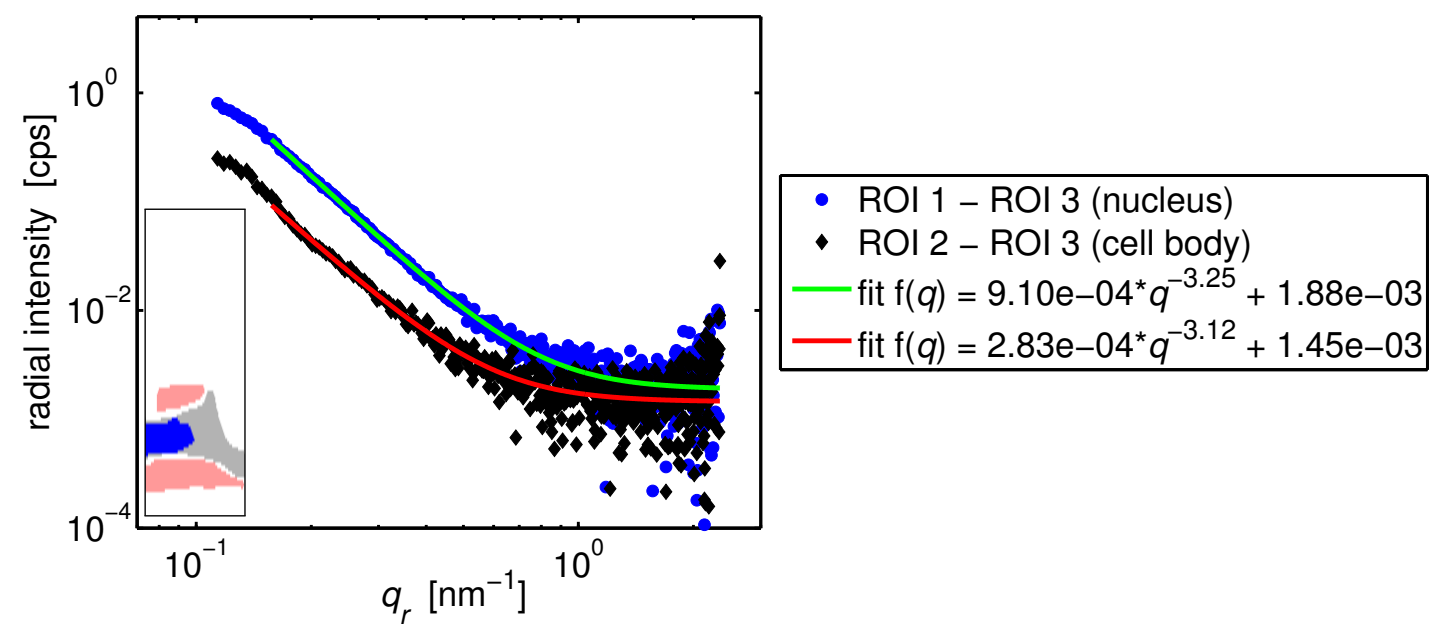

Fig. C.2.: Fitting of the low $q_{r}$-region and high $q_{r}$-region of the background corrected average radial intensity profiles from the nucleus and the cell body with power laws, respectively. The inset shows the regions of the scan that were used for averaging of the scattering patterns (blue: nucleus, ROI 1; gray: cell body, ROI 2; red: empty, ROI 3). The $q_{r}$-ranges over which the fit functions are plotted correspond to the regions that were used for fitting. (Sample BW171, position 3; ID13, Nov. 2012) 


\section{C.2. Results from Measurements at the $\mathbf{P} 10$ Beamline}

In this section, additional measurements fixed-hydrated SK8/18-2 cells at the P10 beamline are presented. For each measurement the fluorescence and phase contrast microscopy image and the X-ray dark-field images as well as fits of the radial intensity profiles of averaged scattering patterns with power law functions are shown. The scan parameters for these measurements are listed in Tab.6.3 in section 6.4 .

\section{Sample BW139, Position 3}
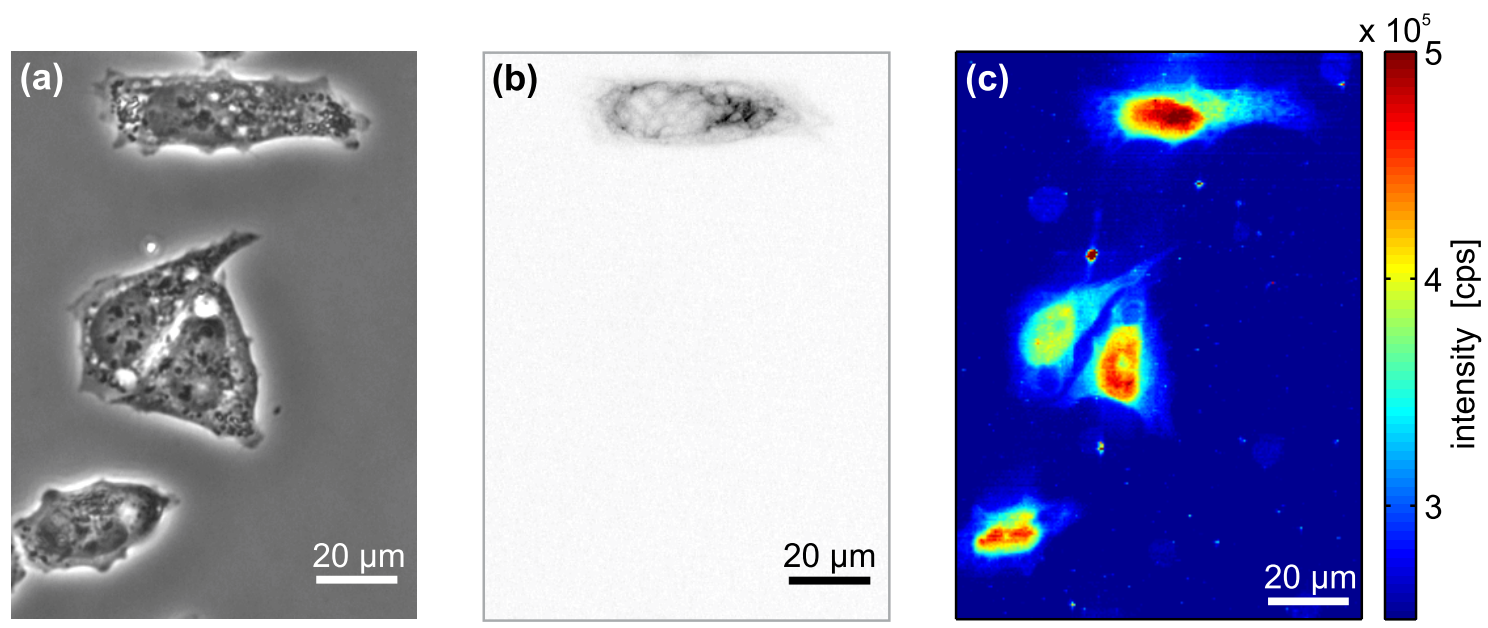

Fig. C.3.: Microscopy images and X-ray dark-field image of a fixed-hydrated SK8/18-2 cell. (a) Visible light phase contrast microscopy image and (b) fluorescence microscopy image of the keratin network in SK8/18-2 cells that were fixed with formaldehyde. (c) X-ray dark-field image of the same sample region as in (a) and (b). (Sample BW139, position 3; P10, Oct. 2012) 


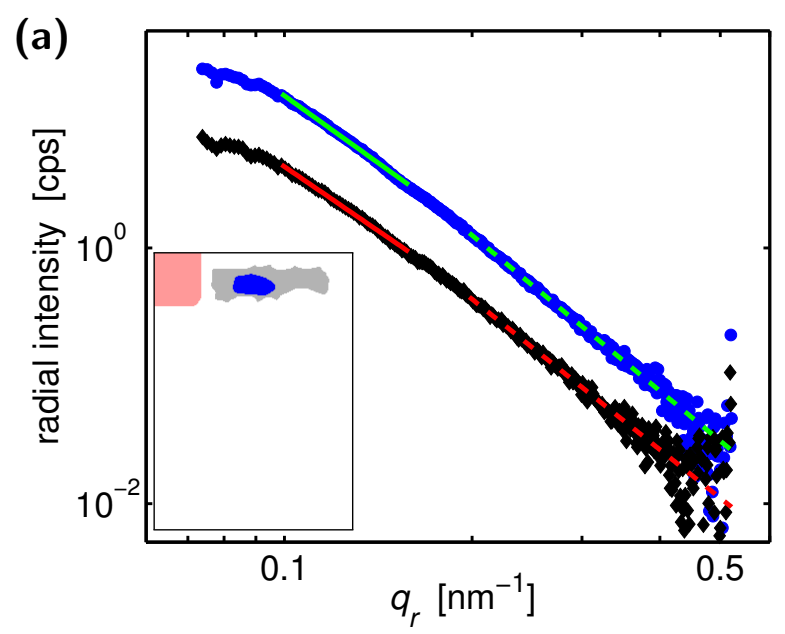

- ROI 1 - ROI 3 (nucleus)

- ROI 2 - ROI 3 (cell body)

- fit $f(q)=4.96 \mathrm{e}-03^{*} q^{-3.50}+1.09 \mathrm{e}-09$

— fit $\mathrm{f}(q)=1.99 \mathrm{e}-03^{*} q^{-3.34}+1.39 \mathrm{e}-06$

- - - fit $f(q)=1.88 \mathrm{e}-03^{*} q^{-4.04}+2.01 \mathrm{e}-10$

- - - fit $f(q)=7.35 e-04^{*} q^{-3.91}+3.13 e-09$

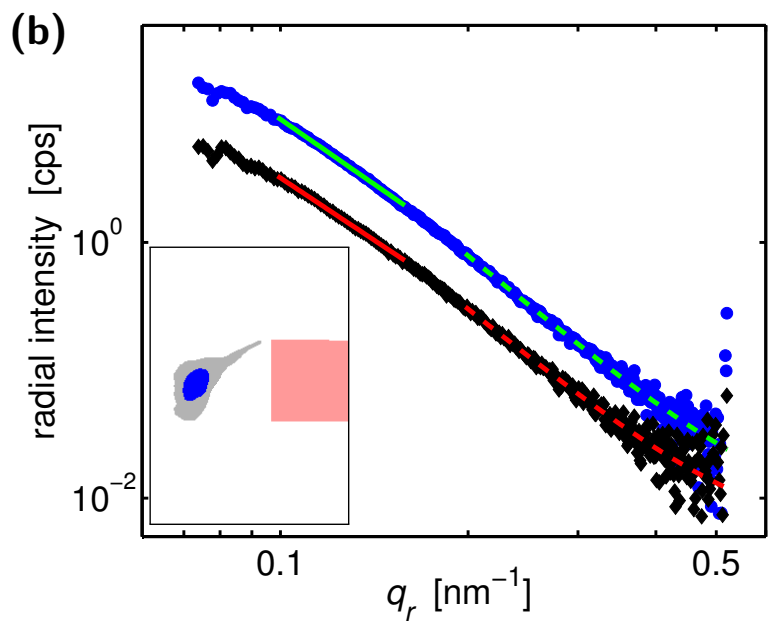

- ROI 1 - ROI 3 (nucleus)

- ROI 2 - ROI 3 (cell body)

- fit $\mathrm{f}(q)=3.83 \mathrm{e}-03^{*} q^{-3.38}+1.80 \mathrm{e}-08$

- fit $f(q)=1.83 e-03^{*} q^{-3.24}+2.10 e-09$

$=--$ fit $f(q)=1.25 e-03^{*} q^{-4.00}+7.00 e-03$

- - - fit $\mathrm{f}(q)=5.43 \mathrm{e}-04^{*} q^{-3.91}+4.95 \mathrm{e}-03$

(c)

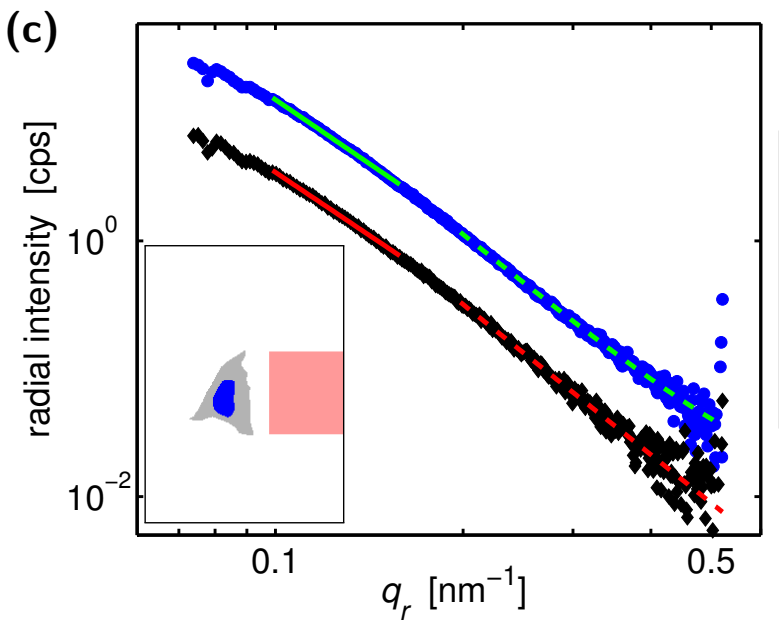

- ROI 1 - ROI 3 (nucleus)

- ROI 2 - ROI 3 (cell body)

- fit $\mathrm{f}(q)=6.01 \mathrm{e}-03^{*} q^{-3.33}+1.61 \mathrm{e}-08$

- fit $\mathrm{f}(q)=1.83 \mathrm{e}-03^{*} q^{-3.27}+1.98 \mathrm{e}-07$

$=--$ fit $f(q)=1.99 e-03^{*} q^{-3.93}+1.02 e-02$

- - - fit $\mathrm{f}(q)=5.94 \mathrm{e}-04^{*} q^{-3.90}+6.67 \mathrm{e}-09$

Fig. C.4.: Fitting of the low $q_{r}$-region and high $q_{r}$-region of the background corrected average radial intensity profiles from the nucleus and the cell body with power laws, respectively. The inset shows the regions of the scan that were used for averaging of the scattering patterns (blue: nucleus, ROI 1; gray: cell body, ROI 2; red: empty, ROI 3). The $q_{r}$-ranges over which the fit functions are plotted correspond to the regions that were used for fitting. (Sample BW139, position 3; P10, Oct. 2012) 


\section{Sample BW145, Position 2}
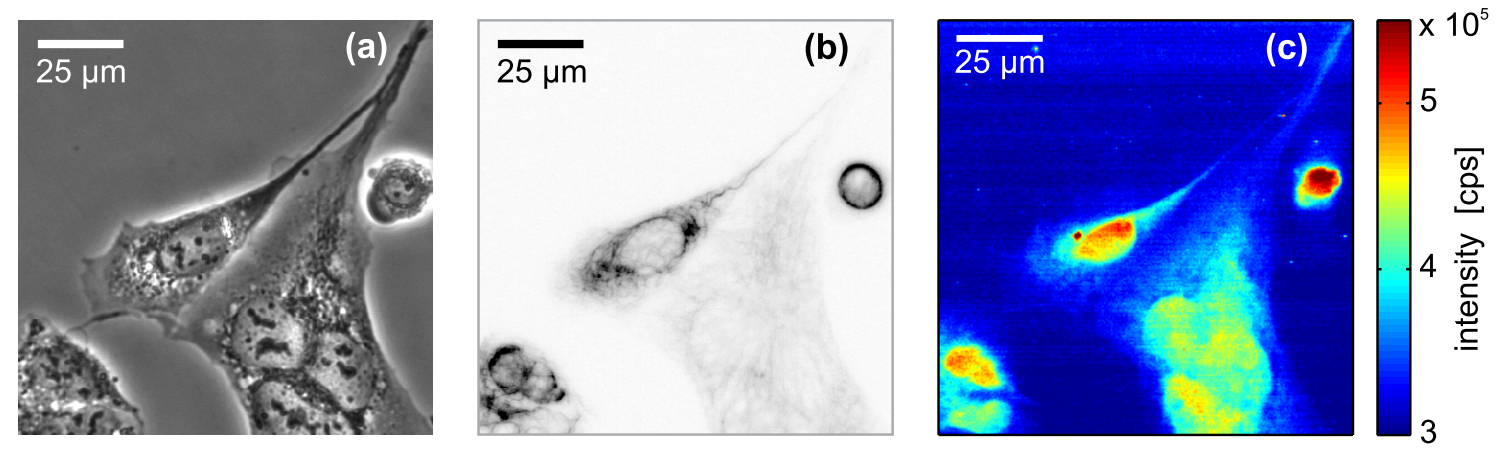

Fig. C.5.: Microscopy images and X-ray dark-field image of a fixed-hydrated SK8/18-2 cell. (a) Visible light phase contrast microscopy image and (b) fluorescence microscopy image of the keratin network in SK8/18-2 cells that were fixed with formaldehyde. (c) Xray dark-field image of the same sample region as in (a) and (b). For better comparability, the dark-field image is normalized on the transmission of one attenuator. (Sample BW145, position 2; P10, Oct. 2012)

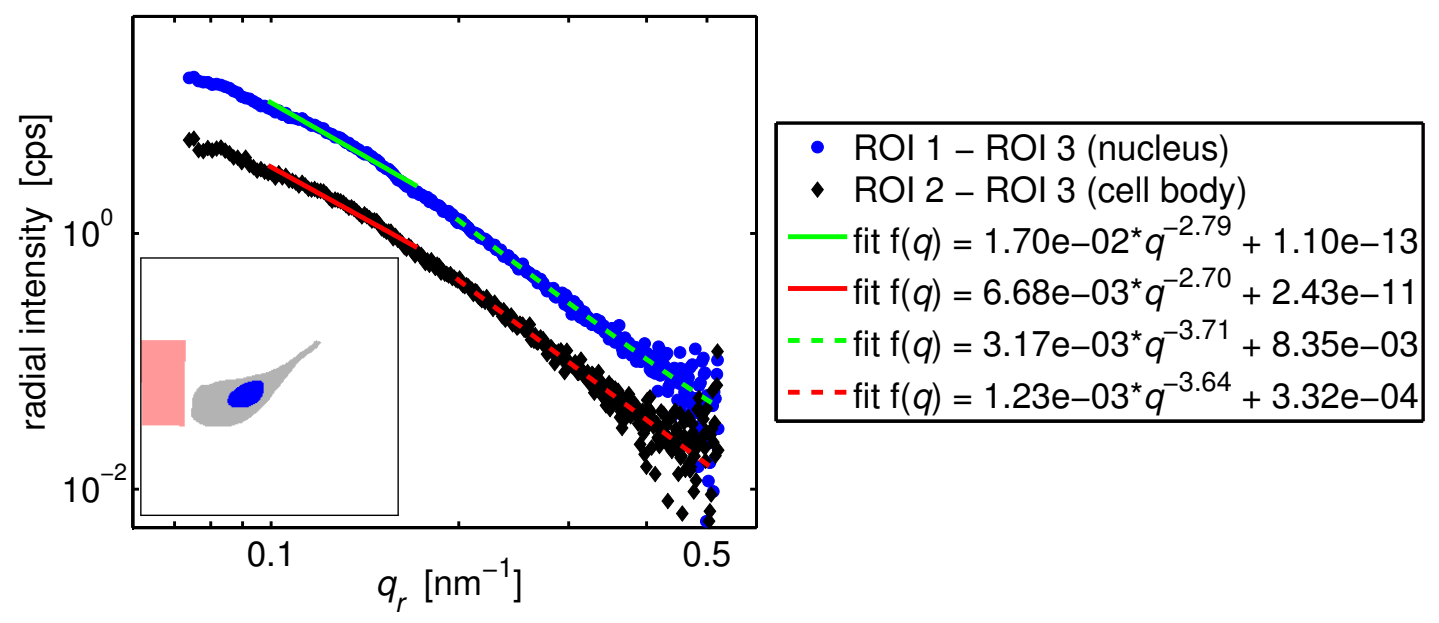

Fig. C.6.: Fitting of the low $q_{r}$-region and high $q_{r}$-region of the background corrected average radial intensity profiles from the nucleus and the cell body with power laws, respectively. The inset shows the regions of the scan that were used for averaging of the scattering patterns (blue: nucleus, ROI 1; gray: cell body, ROI 2; red: empty, ROI 3). The $q_{r}$-ranges over which the fit functions are plotted correspond to the regions that were used for fitting. For better comparability, the radial intensity is normalized on the transmission of one attenuator. (Sample BW145, position 2; P10, Oct. 2012) 


\section{Sample BW145, Position 3}
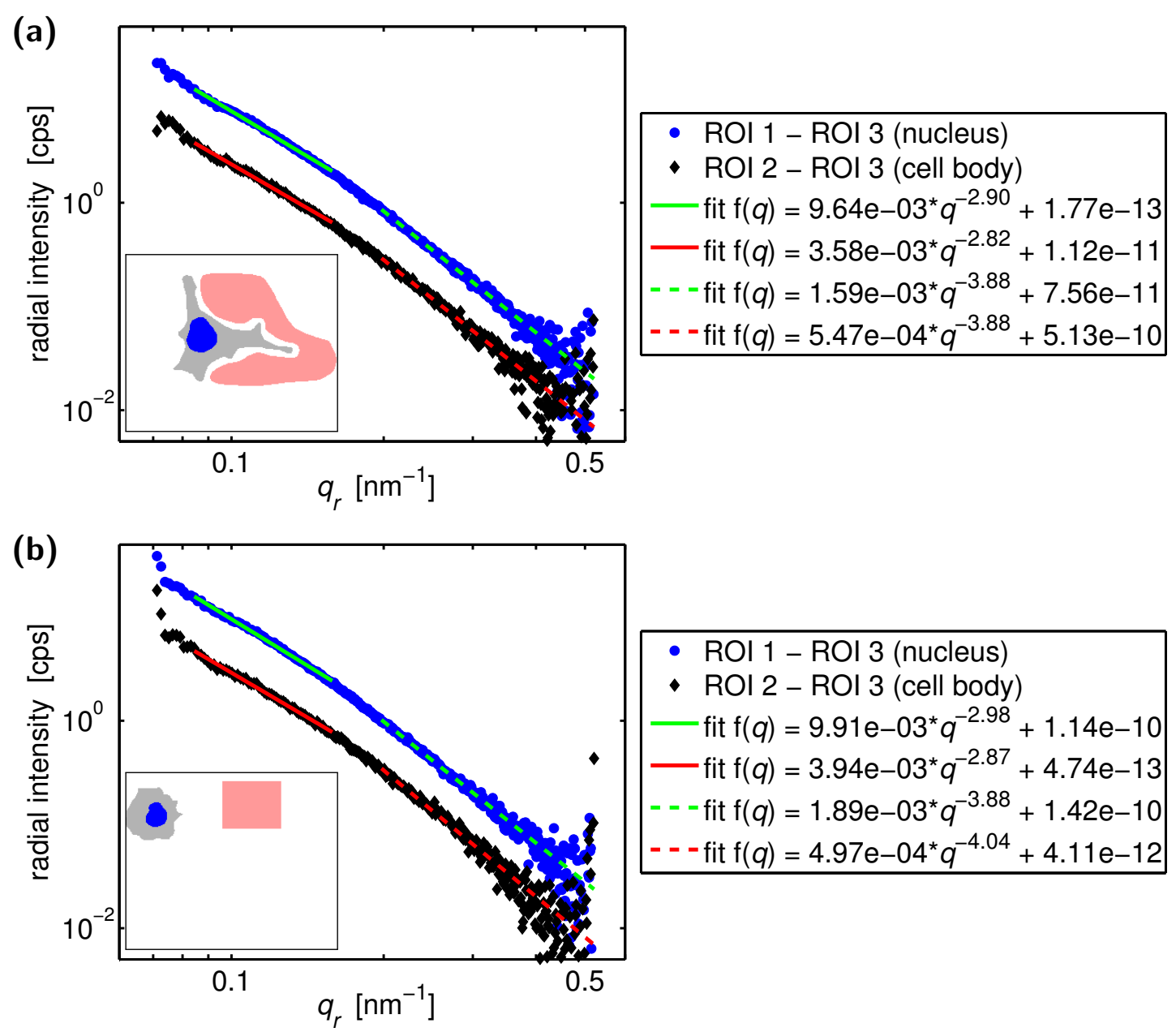

Fig. C.7.: Fitting of the low $q_{r}$-region and high $q_{r}$-region of the background corrected average radial intensity profiles from the nucleus and the cell body with power laws, respectively. The inset shows the regions of the scan that were used for averaging of the scattering patterns (blue: nucleus, ROI 1; gray: cell body, ROI 2; red: empty, ROI 3). The $q_{r}$-ranges over which the fit functions are plotted correspond to the regions that were used for fitting. For better comparability, the radial intensity is normalized on the transmission of one attenuator. (Sample BW145, position 3; P10, Oct. 2012) 

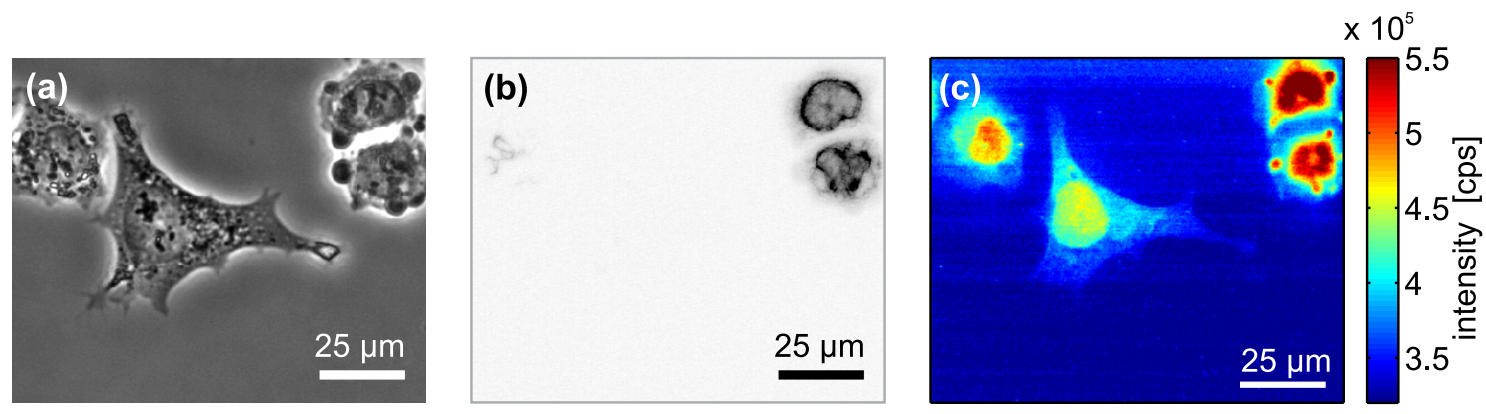

Fig. C.8.: Microscopy images and X-ray dark-field image of a fixed-hydrated SK8/18-2 cell. (a) Visible light phase contrast microscopy image and (b) fluorescence microscopy image of the keratin network in SK8/18-2 cells that were fixed with formaldehyde. (c) Xray dark-field image of the same sample region as in (a) and (b). For better comparability, the dark-field image is normalized on the transmission of one attenuator. (Sample BW145, position 3; P10, Oct. 2012)

\section{Sample BW145, Position 4}
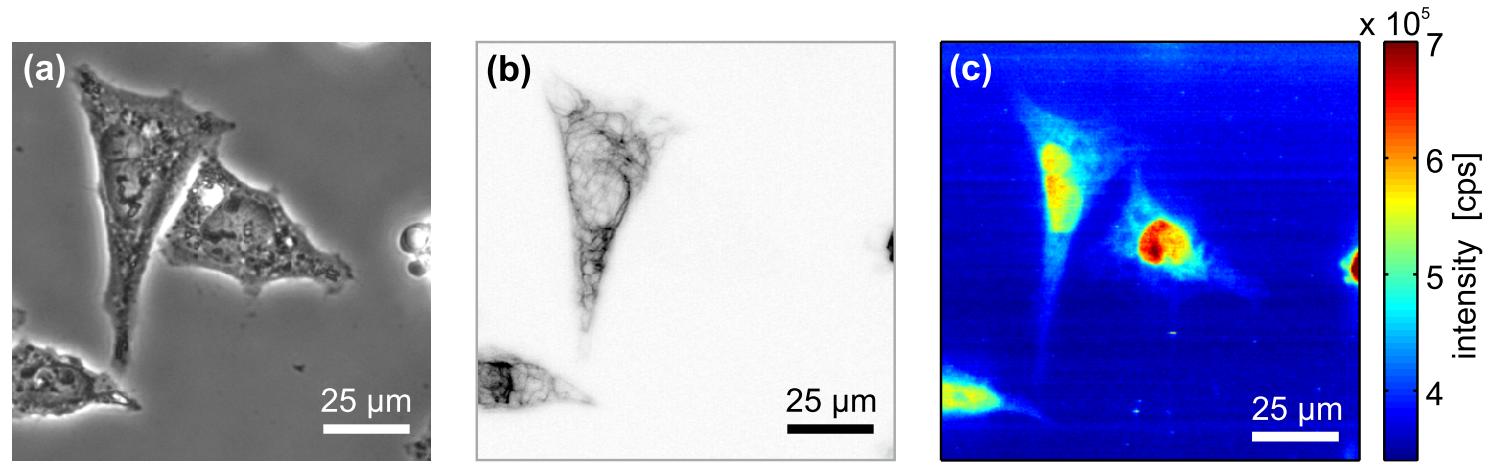

Fig. C.9.: Microscopy images and X-ray dark-field image of a fixed-hydrated SK8/18-2 cell. (a) Visible light phase contrast microscopy image and (b) fluorescence microscopy image of the keratin network in SK8/18-2 cells that were fixed with formaldehyde. (c) Xray dark-field image of the same sample region as in (a) and (b). For better comparability, the dark-field image is normalized on the transmission of one attenuator. (Sample BW145, position 4; P10, Oct. 2012) 


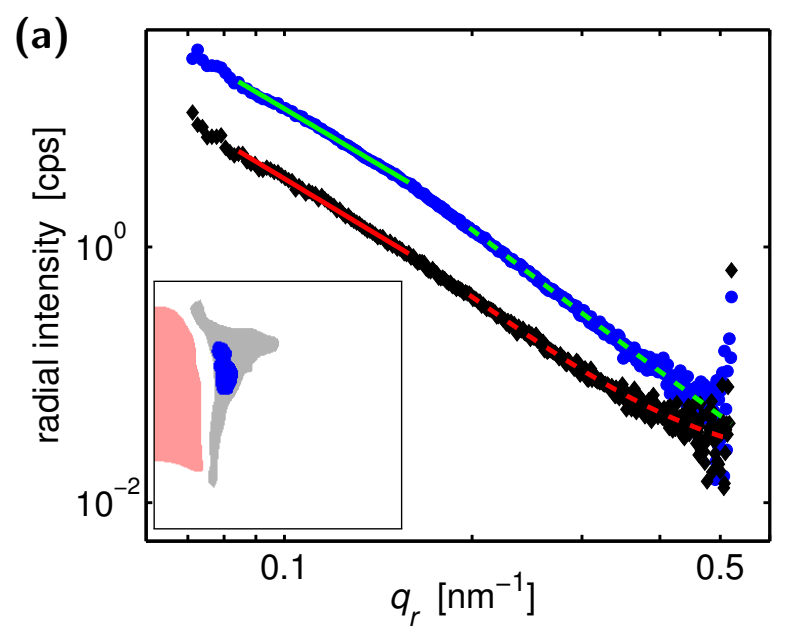

- ROI 1 - ROI 3 (nucleus)

- ROI 2 - ROI 3 (cell body)

fit $\mathrm{f}(q)=1.56 \mathrm{e}-02^{*} q^{-2.89}+8.33 \mathrm{e}-14$

- fit $\mathrm{f}(q)=3.95 \mathrm{e}-03^{*} q^{-2.94}+1.58 \mathrm{e}-11$

- - - fit $f(q)=3.44 \mathrm{e}-03^{*} q^{-3.71}+2.73 \mathrm{e}-03$

--- fit $f(q)=1.19 e-03^{*} q^{-3.59}+1.88 \mathrm{e}-02$

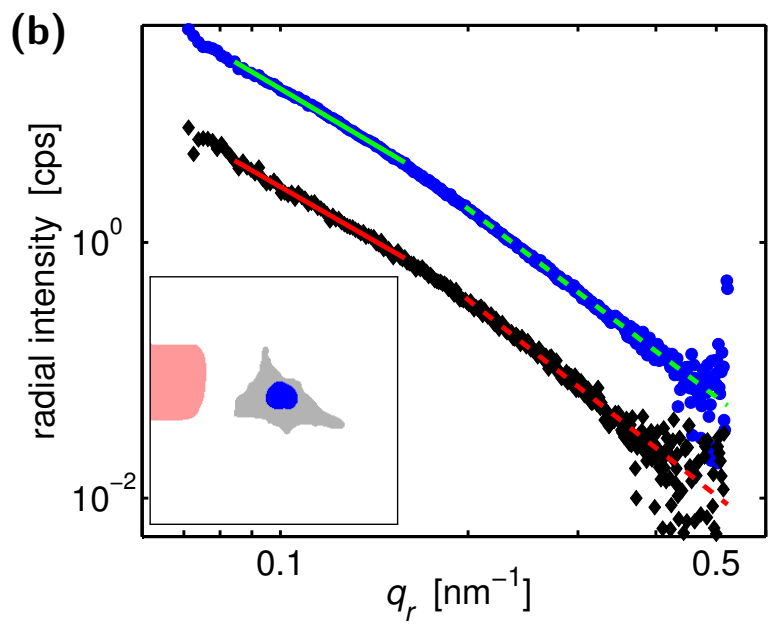

- ROI 1 - ROI 3 (nucleus)

- ROI 2 - ROI 3 (cell body)

fit $f(q)=2.06 \mathrm{e}-02^{*} q^{-2.89}+1.09 \mathrm{e}-13$

- fit $\mathrm{f}(q)=4.30 \mathrm{e}-03^{*} q^{-2.80}+2.99 \mathrm{e}-10$

$=--$ fit $f(q)=4.75 e-03^{*} q^{-3.70}+2.23 e-09$

- - - fit $f(q)=7.25 e-04^{*} q^{-3.85}+9.30 e-10$

Fig. C.10.: Fitting of the low $q_{r}$-region and high $q_{r}$-region of the background corrected average radial intensity profiles from the nucleus and the cell body with power laws, respectively. The inset shows the regions of the scan that were used for averaging of the scattering patterns (blue: nucleus, ROI 1; gray: cell body, ROI 2; red: empty, ROI 3). The $q_{r}$-ranges over which the fit functions are plotted correspond to the regions that were used for fitting. For better comparability, the radial intensity is normalized on the transmission of one attenuator. (Sample BW145, position 4; P10, Oct. 2012) 


\section{Sample BW145, Position 5}
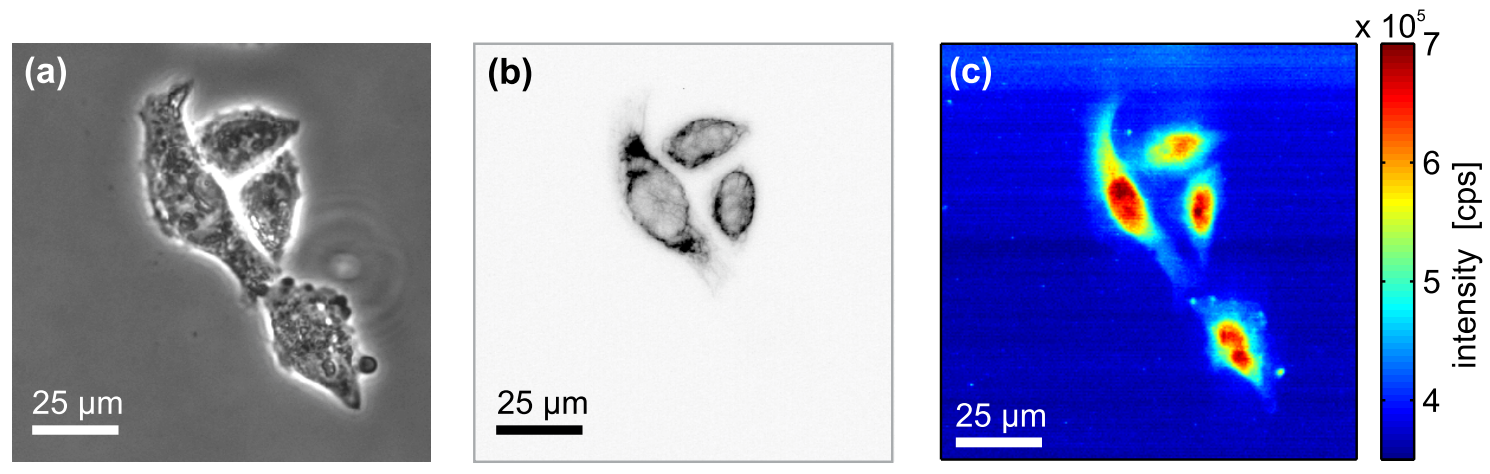

Fig. C.11.: Microscopy images and X-ray dark-field image of a fixed-hydrated SK8/18-2 cell. (a) Visible light phase contrast microscopy image and (b) fluorescence microscopy image of the keratin network in SK8/18-2 cells that were fixed with formaldehyde. (c) Xray dark-field image of the same sample region as in (a) and (b). For better comparability, the dark-field image is normalized on the transmission of one attenuator. (Sample BW145, position 5; P10, Oct. 2012)

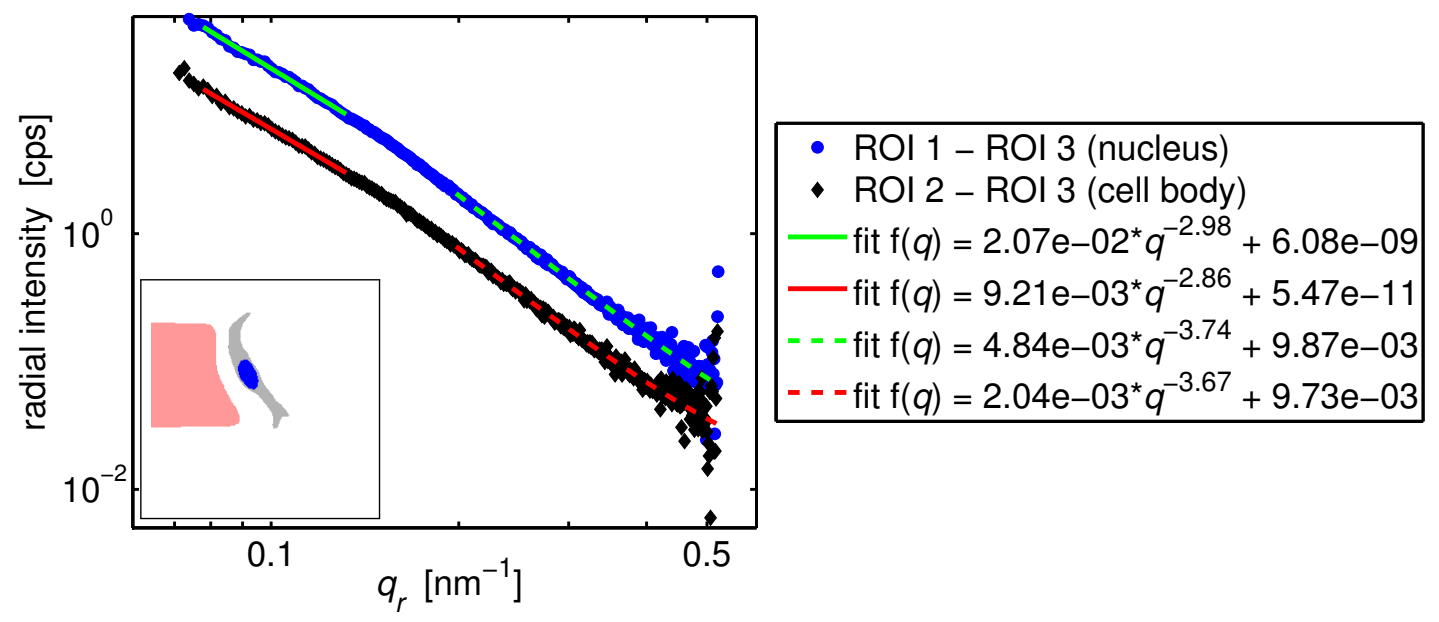

Fig. C.12.: Fitting of the low $q_{r}$-region and high $q_{r}$-region of the background corrected average radial intensity profiles from the nucleus and the cell body with power laws, respectively. The inset shows the regions of the scan that were used for averaging of the scattering patterns (blue: nucleus, ROI 1; gray: cell body, ROI 2; red: empty, ROI 3). The $q_{r}$-ranges over which the fit functions are plotted correspond to the regions that were used for fitting. For better comparability, the radial intensity is normalized on the transmission of one attenuator. (Sample BW145, position 5; P10, Oct. 2012) 


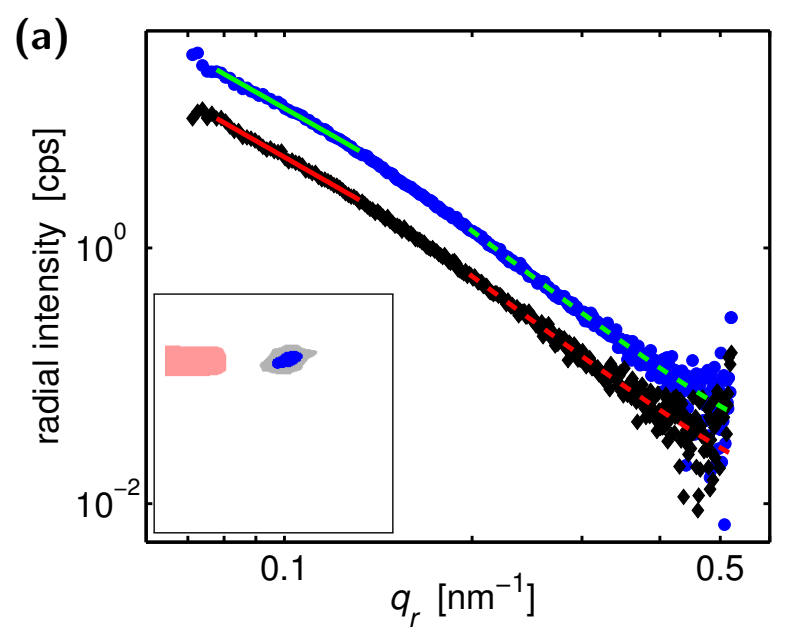

- ROI 1 - ROI 3 (nucleus)

- ROI 2 - ROI 3 (cell body)

fit $f(q)=2.11 e-02^{*} q^{-2.77}+2.39 \mathrm{e}-14$

- fit $f(q)=8.39 \mathrm{e}-03^{*} q^{-2.79}+3.92 \mathrm{e}-10$

- - - fit $f(q)=3.32 e-03^{*} q^{-3.73}+1.38 \mathrm{e}-02$

- - - fit $f(q)=1.72 e-03^{*} q^{-3.63}+6.09 e-03$

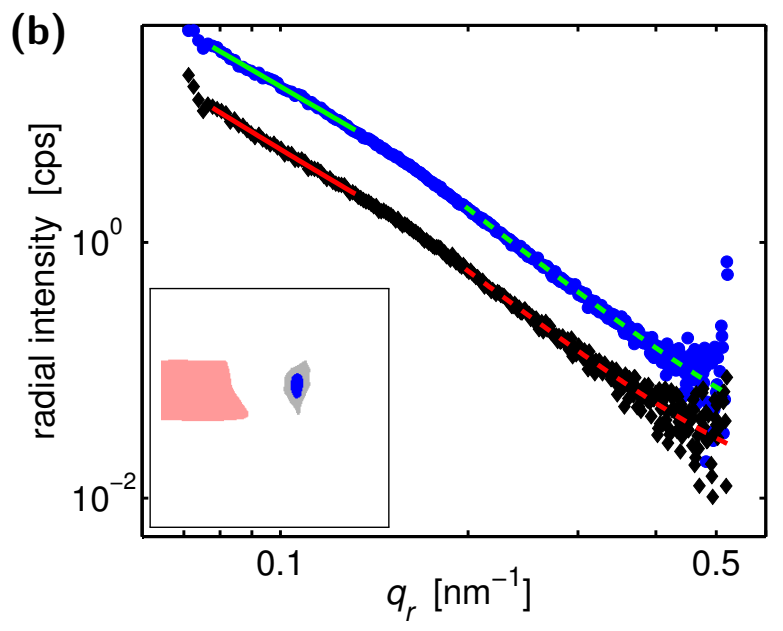

- ROI 1 - ROI 3 (nucleus)

- ROI 2 - ROI 3 (cell body)

- fit $f(q)=2.40 e-02^{*} q^{-2.84}+1.15 e-09$

- fit $\mathrm{f}(q)=4.85 \mathrm{e}-03^{*} q^{-3.03}+1.44 \mathrm{e}-01$

--- fit $f(q)=4.22 \mathrm{e}-03^{*} q^{-3.77}+1.63 \mathrm{e}-02$

- - - fit $f(q)=1.59 e-03^{*} q^{-3.67}+9.15 e-03$

(c)

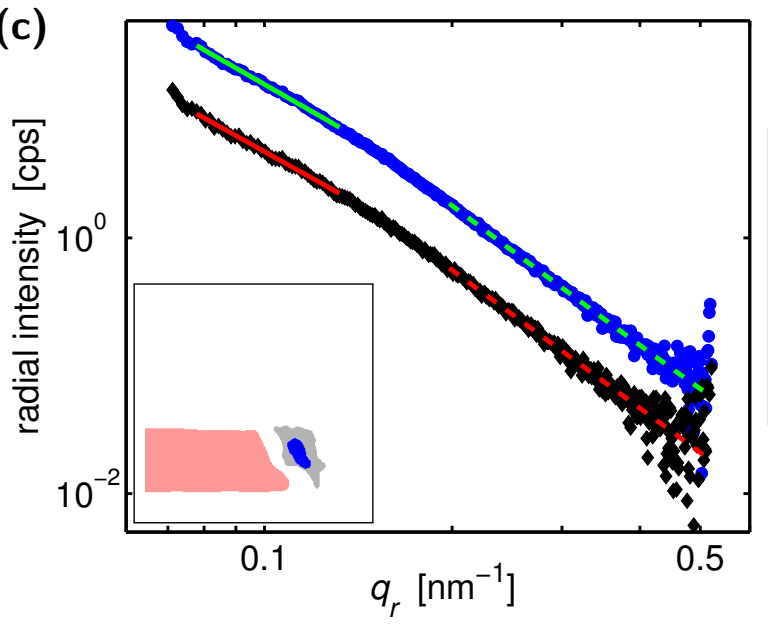

- ROI 1 - ROI 3 (nucleus)

- ROI 2 - ROI 3 (cell body)

fit $\mathrm{f}(q)=2.59 \mathrm{e}-02^{\star} q^{-2.79}+3.88 \mathrm{e}-12$

— fit $\mathrm{f}(q)=9.00 \mathrm{e}-03^{*} q^{-2.72}+4.29 \mathrm{e}-10$

- - - fit $f(q)=4.90 \mathrm{e}-03^{*} q^{-3.66}+3.86 \mathrm{e}-03$

--- fit $\mathrm{f}(q)=1.75 \mathrm{e}-03^{*} q^{-3.58}+1.80 \mathrm{e}-08$

Fig. C.13.: Fitting of the low $q_{r}$-region and high $q_{r}$-region of the background corrected average radial intensity profiles from the nucleus and the cell body with power laws, respectively. The inset shows the regions of the scan that were used for averaging of the scattering patterns (blue: nucleus, ROI 1; gray: cell body, ROI 2; red: empty, ROI 3). The $q_{r}$-ranges over which the fit functions are plotted correspond to the regions that were used for fitting. For better comparability, the radial intensity is normalized on the transmission of one attenuator. (Sample BW145, position 5; P10, Oct. 2012) 


\section{Sample BW156, Position 3}
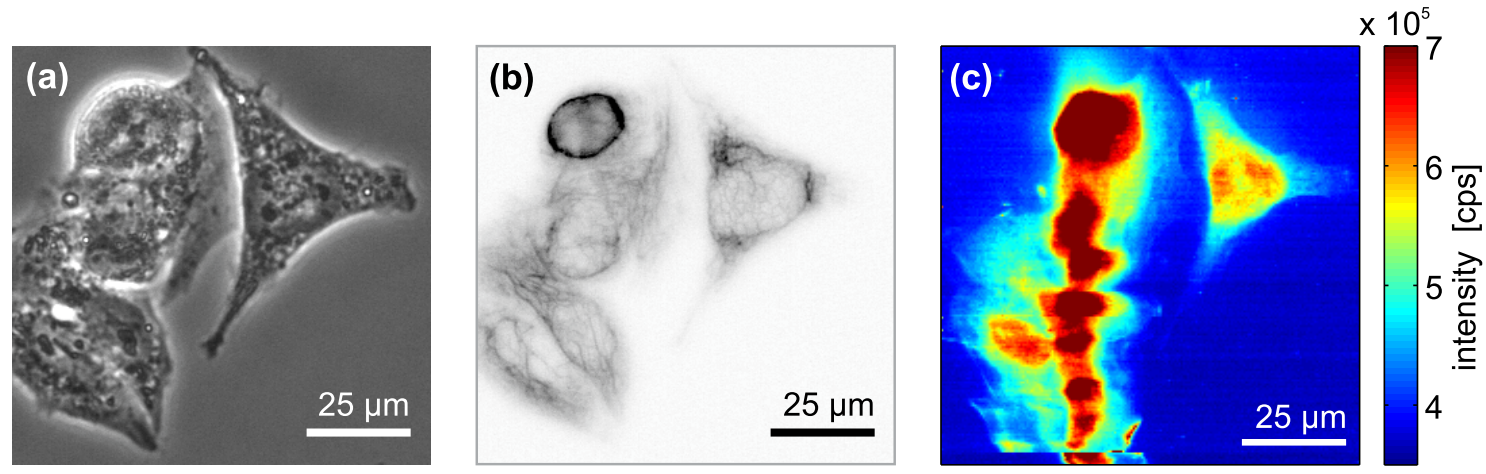

Fig. C.14.: Microscopy images and X-ray dark-field image of a fixed-hydrated SK8/18-2 cell. (a) Visible light phase contrast microscopy image and (b) fluorescence microscopy image of the keratin network in SK8/18-2 cells that were fixed with formaldehyde. (c) X-ray dark-field image of the same sample region as in (a) and (b). (Sample BW156, position 3; P10, Oct. 2012)

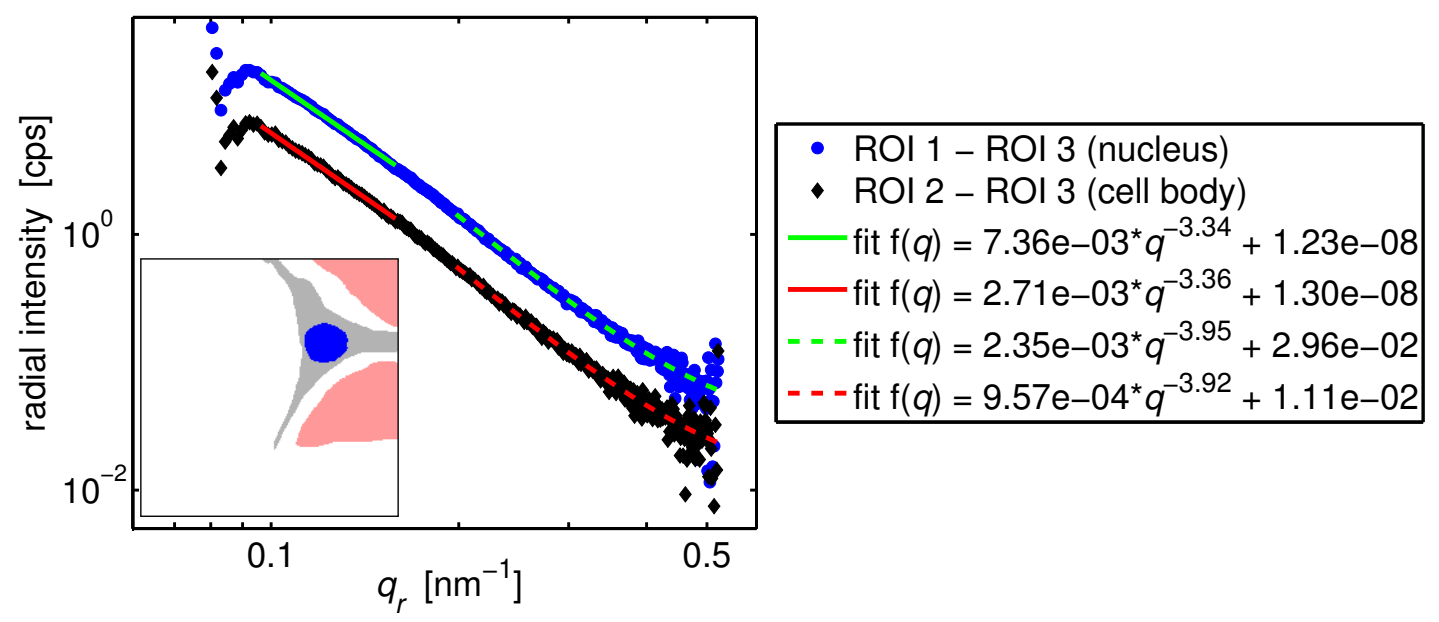

Fig. C.15.: Fitting of the low $q_{r}$-region and high $q_{r}$-region of the background corrected average radial intensity profiles from the nucleus and the cell body with power laws, respectively. The inset shows the regions of the scan that were used for averaging of the scattering patterns (blue: nucleus, ROI 1; gray: cell body, ROI 2; red: empty, ROI 3). The $q_{r}$-ranges over which the fit functions are plotted correspond to the regions that were used for fitting. (Sample BW156, position 3; P10, Oct. 2012) 


\section{Sample BW235, Position 1}

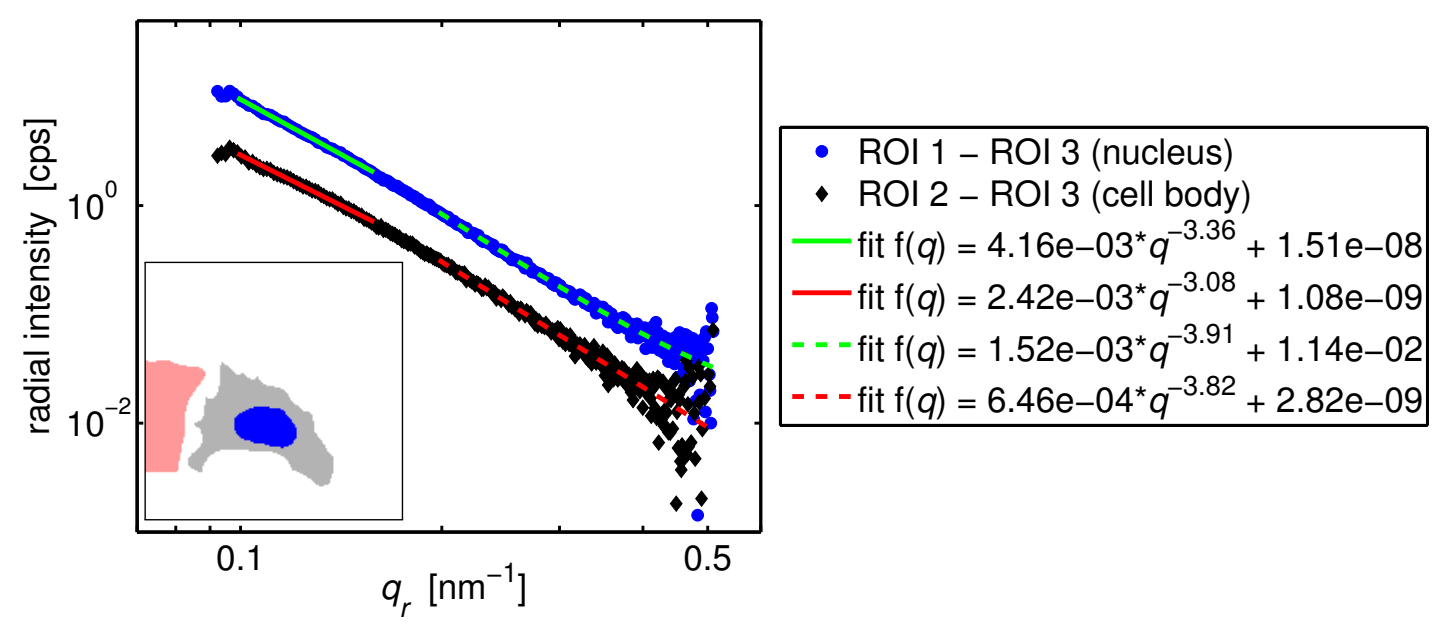

Fig. C.16.: Fitting of the low $q_{r}$-region and high $q_{r}$-region of the background corrected average radial intensity profiles from the nucleus and the cell body with power laws, respectively. The inset shows the regions of the scan that were used for averaging of the scattering patterns (blue: nucleus, ROI 1; gray: cell body, ROI 2; red: empty, ROI 3). The $q_{r}$-ranges over which the fit functions are plotted correspond to the regions that were used for fitting. (Sample BW235, position 1; P10, March 2013)

\section{Sample BW237, Position 1}
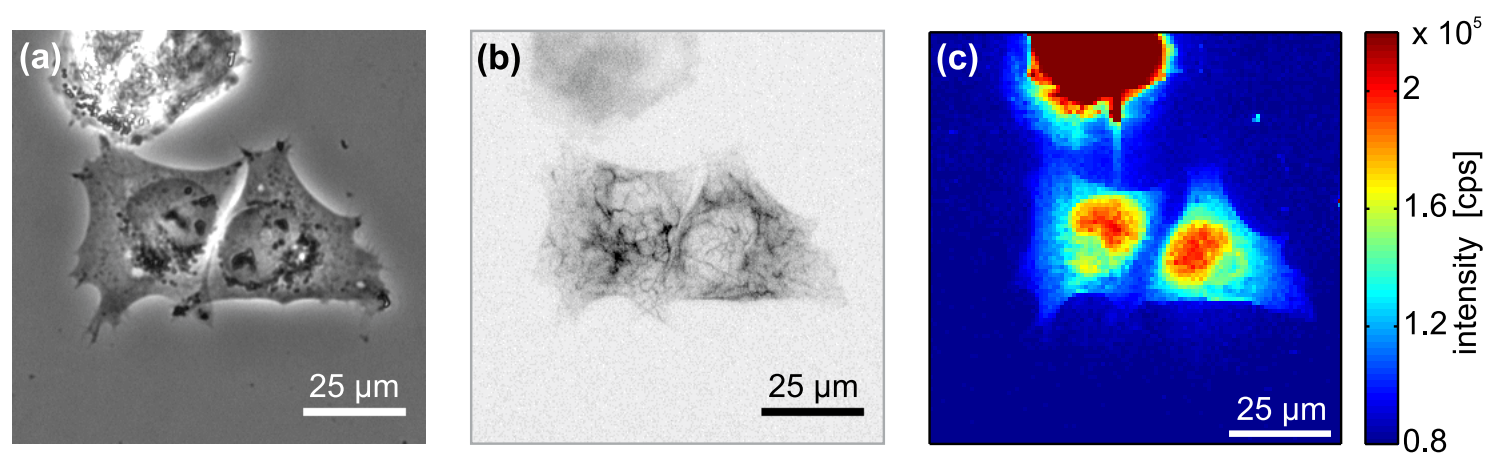

Fig. C.17.: Microscopy images and X-ray dark-field image of a fixed-hydrated SK8/18-2 cell. (a) Visible light phase contrast microscopy image and (b) fluorescence microscopy image of the keratin network in SK8/18-2 cells that were fixed with formaldehyde. (c) X-ray dark-field image of the same sample region as in (a) and (b). (Sample BW237, position 1; P10, March 2013) 


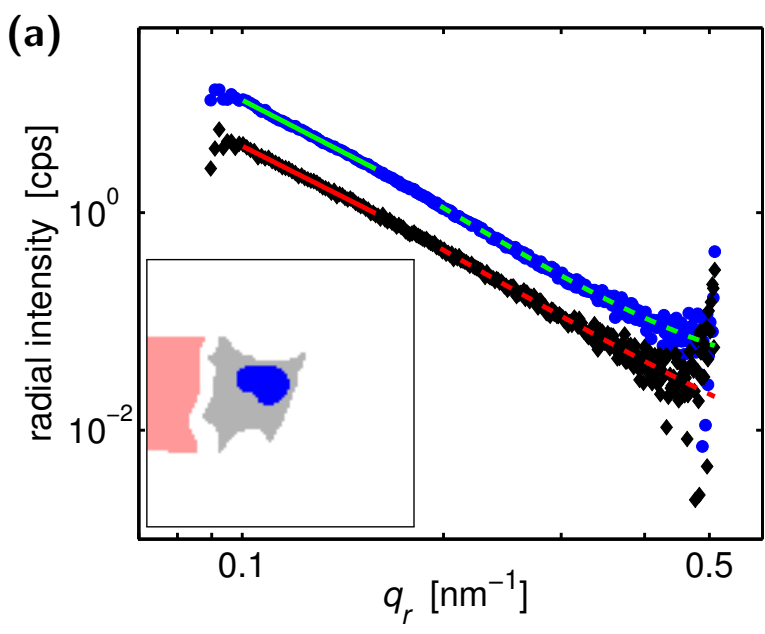

- ROI 1 - ROI 3 (nucleus)

- ROI 2 - ROI 3 (cell body)

- fit $\mathrm{f}(q)=7.29 \mathrm{e}-03^{*} q^{-3.17}+1.09 \mathrm{e}-09$

- fit $\mathrm{f}(q)=3.23 \mathrm{e}-03^{*} q^{-3.10}+7.92 \mathrm{e}-10$

- - - fit $f(q)=2.61 \mathrm{e}-03^{*} q^{-3.74}+2.73 \mathrm{e}-02$

--- fit $f(q)=1.61 \mathrm{e}-03^{*} q^{-3.50}+3.27 \mathrm{e}-03$

(b)

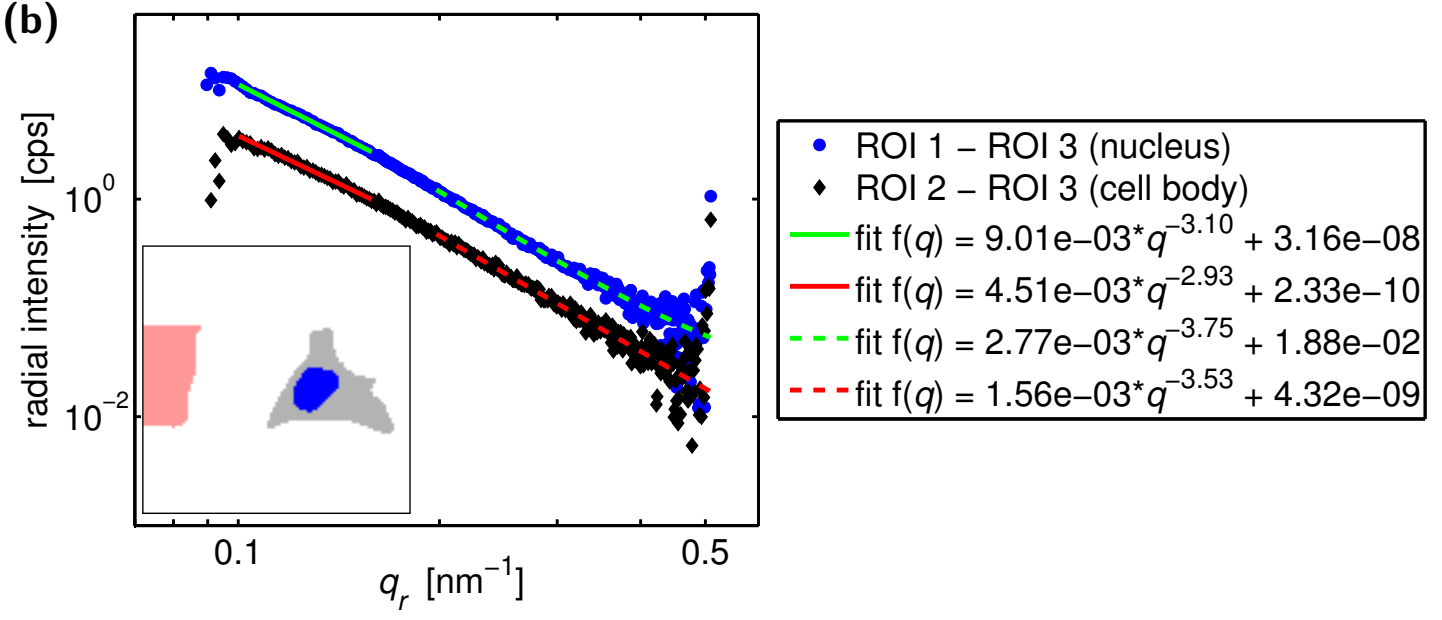

Fig. C.18.: Fitting of the low $q_{r}$-region and high $q_{r}$-region of the background corrected average radial intensity profiles from the nucleus and the cell body with power laws, respectively. The inset shows the regions of the scan that were used for averaging of the scattering patterns (blue: nucleus, ROI 1; gray: cell body, ROI 2; red: empty, ROI 3). The $q_{r}$-ranges over which the fit functions are plotted correspond to the regions that were used for fitting. (Sample BW237, position 1; P10, March 2013) 


\section{Sample BW237, Position 2}
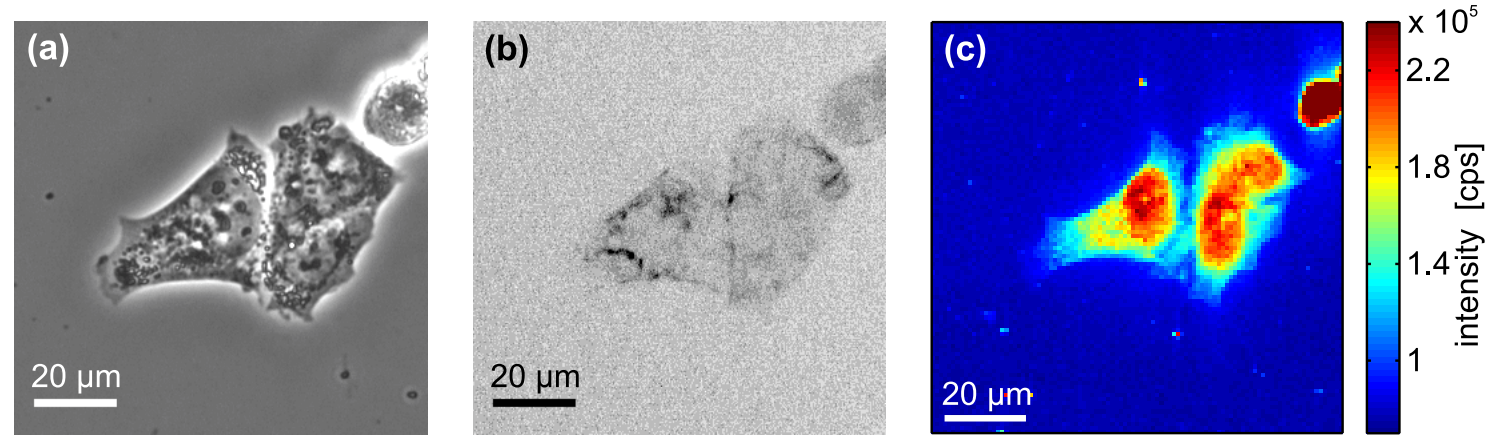

Fig. C.19.: Microscopy images and X-ray dark-field image of a fixed-hydrated SK8/18-2 cell. (a) Visible light phase contrast microscopy image and (b) fluorescence microscopy image of the keratin network in SK8/18-2 cells that were fixed with formaldehyde. (c) X-ray dark-field image of the same sample region as in (a) and (b). (Sample BW237, position 2; P10, March 2013)

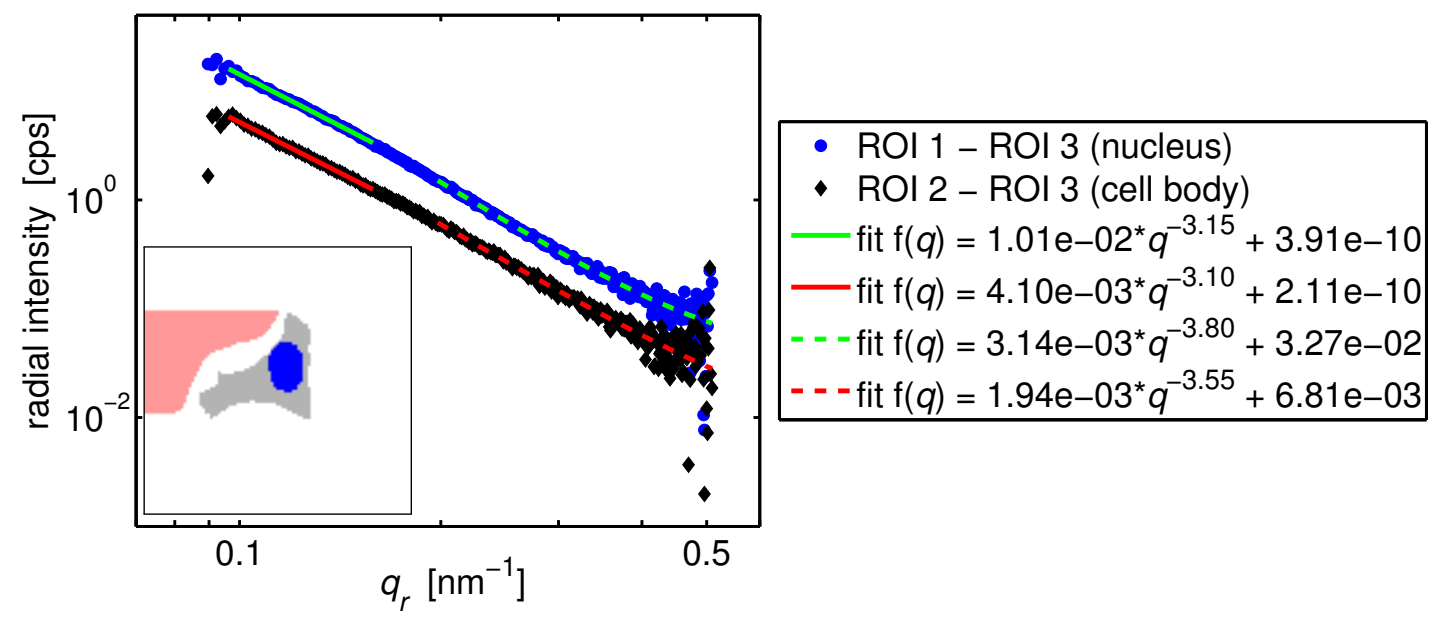

Fig. C.20.: Fitting of the low $q_{r}$-region and high $q_{r}$-region of the background corrected average radial intensity profiles from the nucleus and the cell body with power laws, respectively. The inset shows the regions of the scan that were used for averaging of the scattering patterns (blue: nucleus, ROI 1; gray: cell body, ROI 2; red: empty, ROI 3). The $q_{r}$-ranges over which the fit functions are plotted correspond to the regions that were used for fitting. (Sample BW237, position 2; P10, March 2013) 


\section{Sample BW237, Position 3}
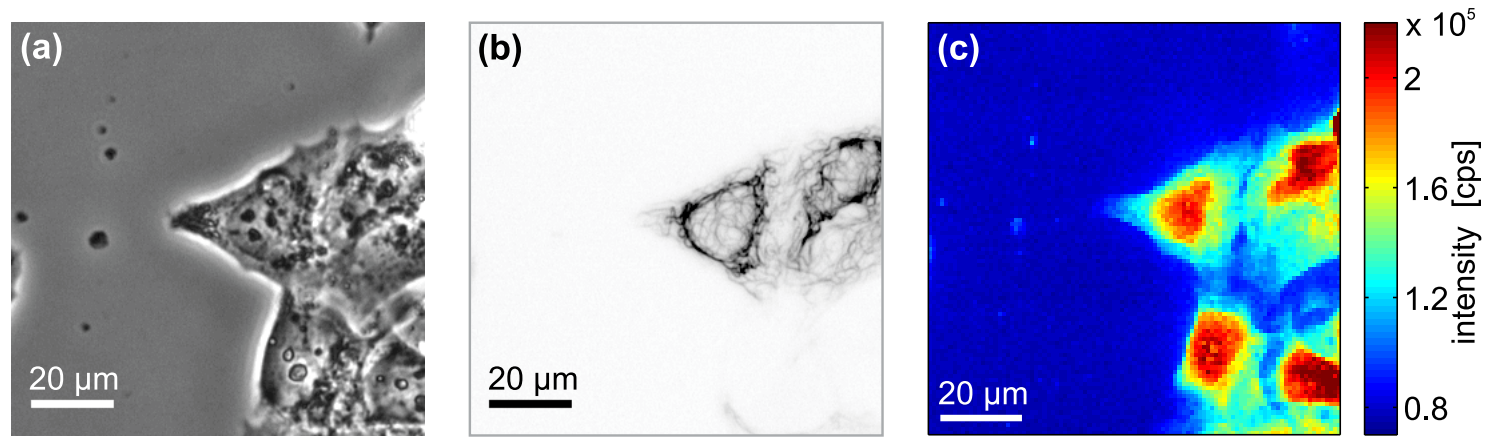

Fig. C.21.: Microscopy images and X-ray dark-field image of a fixed-hydrated SK8/18-2 cell. (a) Visible light phase contrast microscopy image and (b) fluorescence microscopy image of the keratin network in SK8/18-2 cells that were fixed with formaldehyde. (c) X-ray dark-field image of the same sample region as in (a) and (b). (Sample BW237, position 3; P10, March 2013)

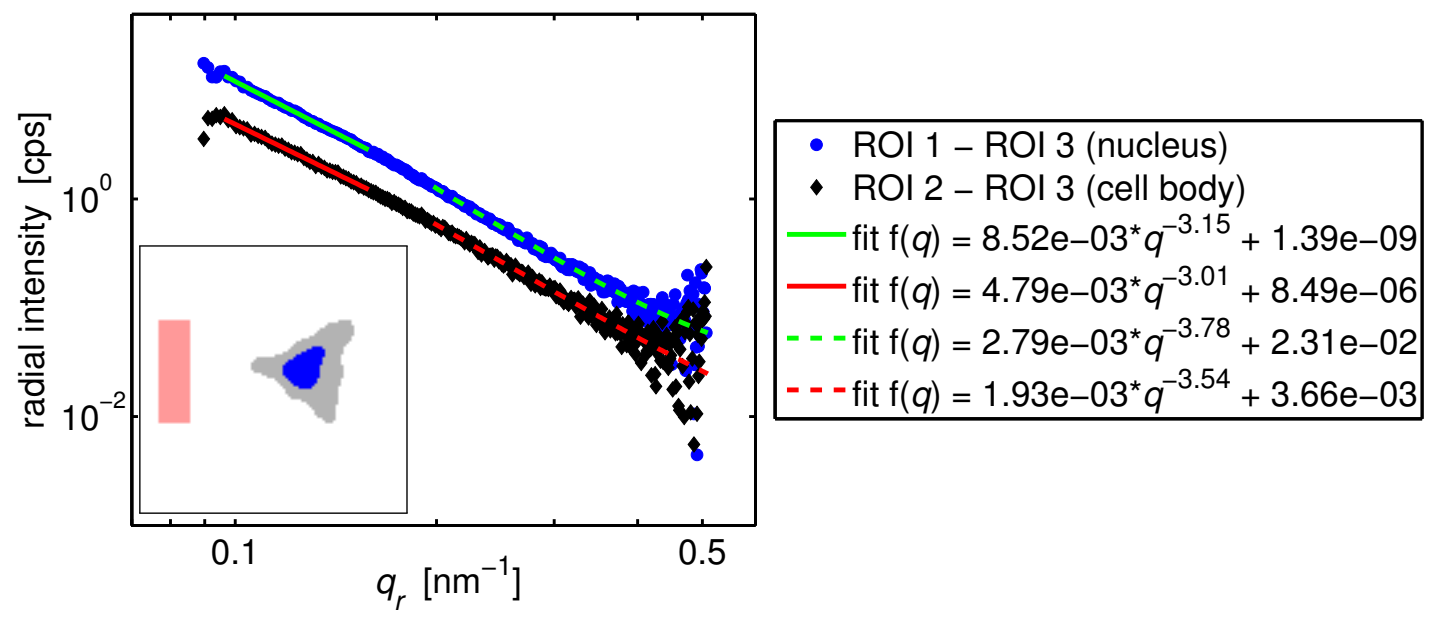

Fig. C.22.: Fitting of the low $q_{r}$-region and high $q_{r}$-region of the background corrected average radial intensity profiles from the nucleus and the cell body with power laws, respectively. The inset shows the regions of the scan that were used for averaging of the scattering patterns (blue: nucleus, ROI 1; gray: cell body, ROI 2; red: empty, ROI 3). The $q_{r}$-ranges over which the fit functions are plotted correspond to the regions that were used for fitting. (Sample BW237, position 3; P10, March 2013) 



\section{Supplementary Data on Living Cells}

\section{D.1. Results from Measurements at the P10 Beamline}

In this section, additional measurements on living SK8/18-2 cells at the P10 beamline are presented. For each measurement the bright-field image taken before device assembly, the beamline microscope and the X-ray dark-field images as well as fits of the radial intensity profiles of averaged scattering patterns with power law functions are shown. The scan parameters for these measurements are listed in Tab.7.2 in section 7.3 .

\section{Living Cells II.7, Position 7}

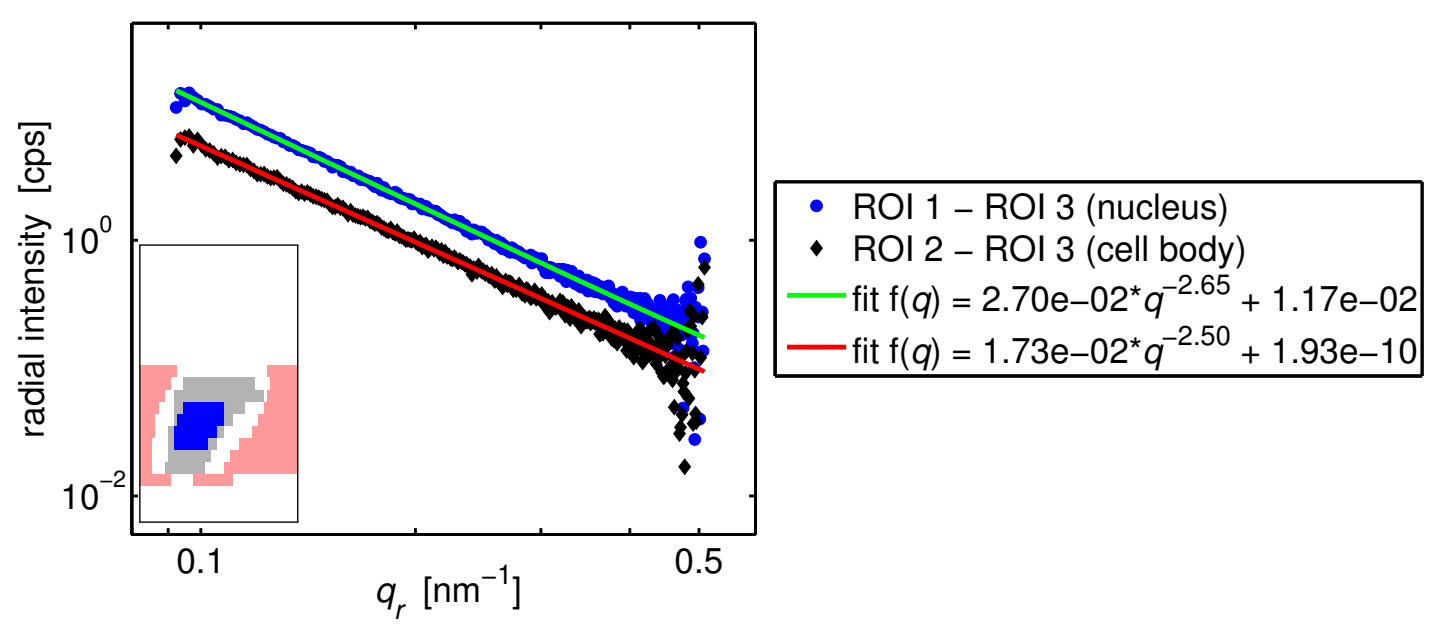

Fig. D.1.: Fitting of the background corrected average radial intensity profiles from the nucleus and the cell body with a power law. The inset shows the regions of the scan that were used for averaging of the scattering patterns (blue: nucleus, ROI 1; gray: cell body, ROI 2 ; red: empty, ROI 3). The $q_{r}$-ranges over which the fit functions are plotted correspond to the regions that were used for fitting. (Live Cells II.7, position 7; P10, March 2013) 


\section{Living Cells II.7, Position 8}
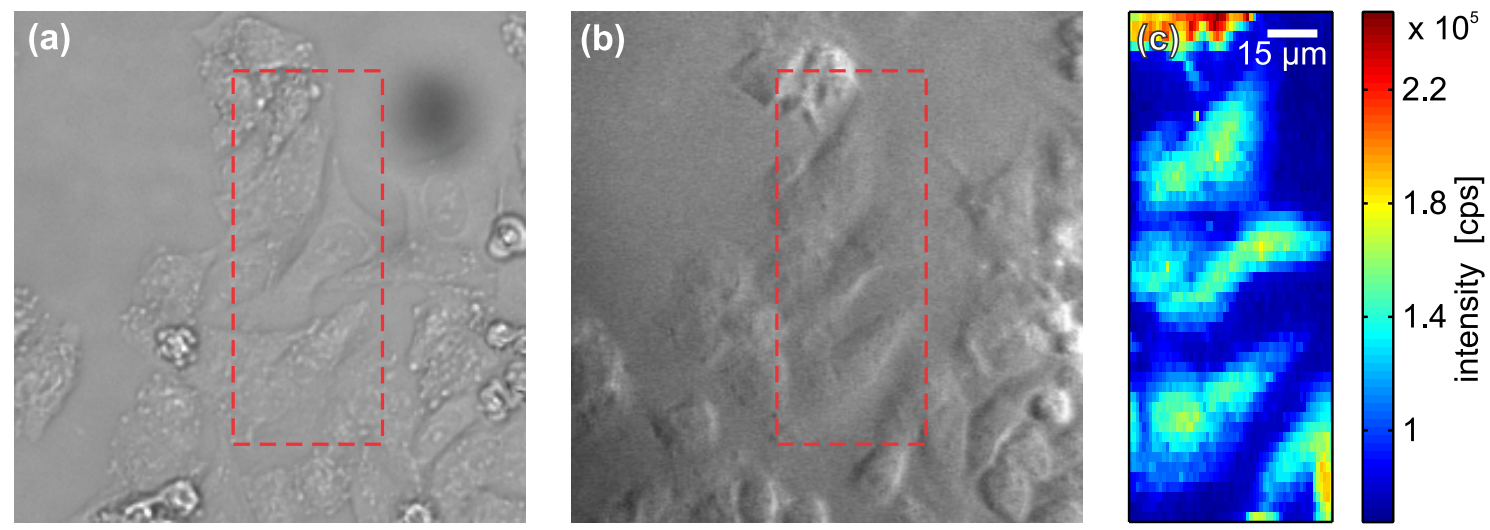

Fig. D.2.: Visible-light microscopy and X-ray dark-field image. (a) Bright-field image of SK8/18-2 cells taken directly before device assembly. (b) Image taken with the beamline microscopy directly before the measurement. (c) X-ray dark-field image taken on the marked region in panel a. (Live Cells II.7, position 8; P10, March 2013)

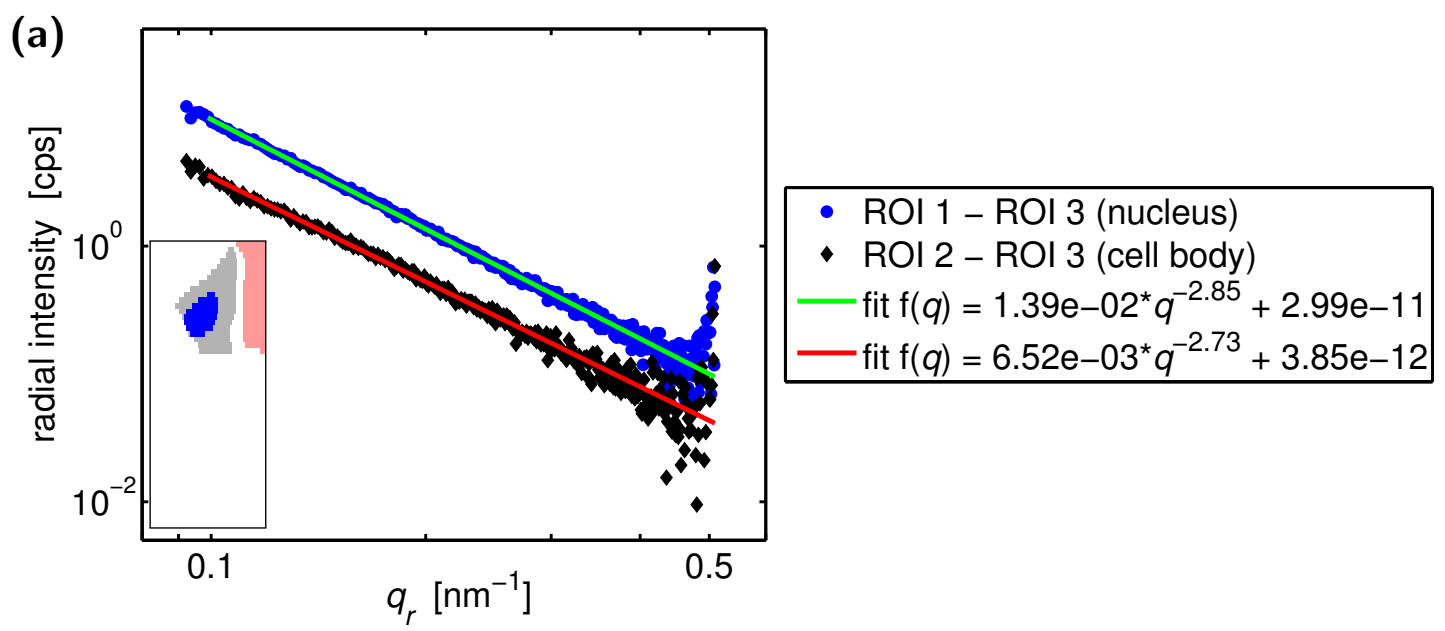

Fig. D.3.: Fitting of the background corrected average radial intensity profiles from the nucleus and the cell body with a power law. The inset shows the regions of the scan that were used for averaging of the scattering patterns (blue: nucleus, ROI 1; gray: cell body, ROI 2; red: empty, ROI 3). The $q_{r}$-ranges over which the fit functions are plotted correspond to the regions that were used for fitting. (Live Cells II.7, position 8; P10, March 2013) 


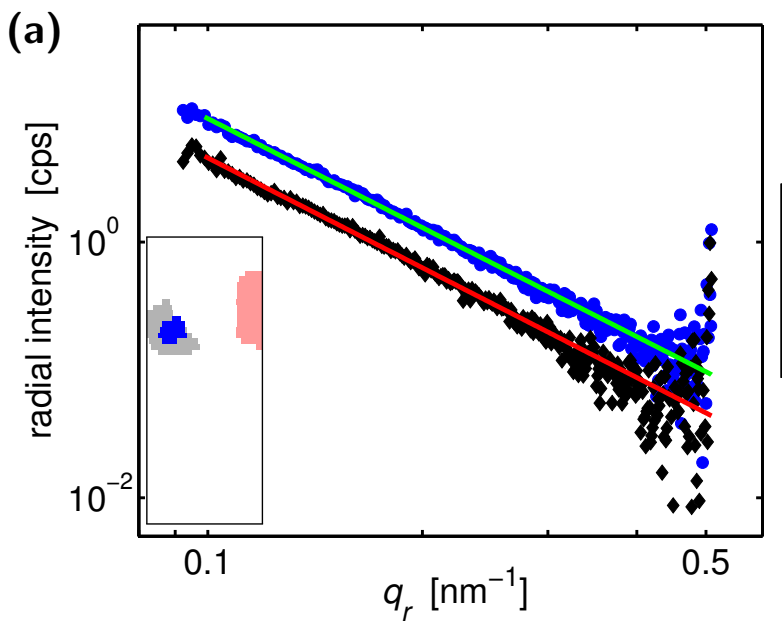

$\begin{aligned} &-\mathrm{ROI} 1-\mathrm{ROI} 3 \text { (nucleus) } \\ &-\mathrm{ROI} 2-\mathrm{ROI} 3 \text { (cell body) } \\ &-\mathrm{fit} f(q)=1.35 \mathrm{e}-02^{*} q^{-2.83}+2.93 \mathrm{e}-11 \\ &-\mathrm{fit} f(q)=6.37 \mathrm{e}-03^{*} q^{-2.85}+5.45 \mathrm{e}-10\end{aligned}$

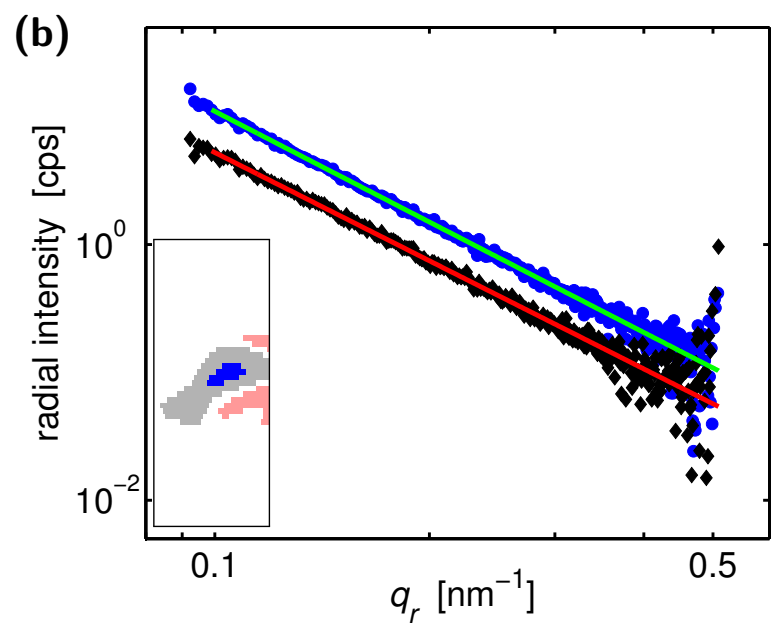

- ROI 1 - ROI 3 (nucleus)

- ROI 2 - ROI 3 (cell body)

- fit $f(q)=1.47 e-02^{*} q^{-2.88}+6.54 e-04$

— fit $\mathrm{f}(q)=8.08 \mathrm{e}-03^{*} q^{-2.81}+1.24 \mathrm{e}-10$

(c)

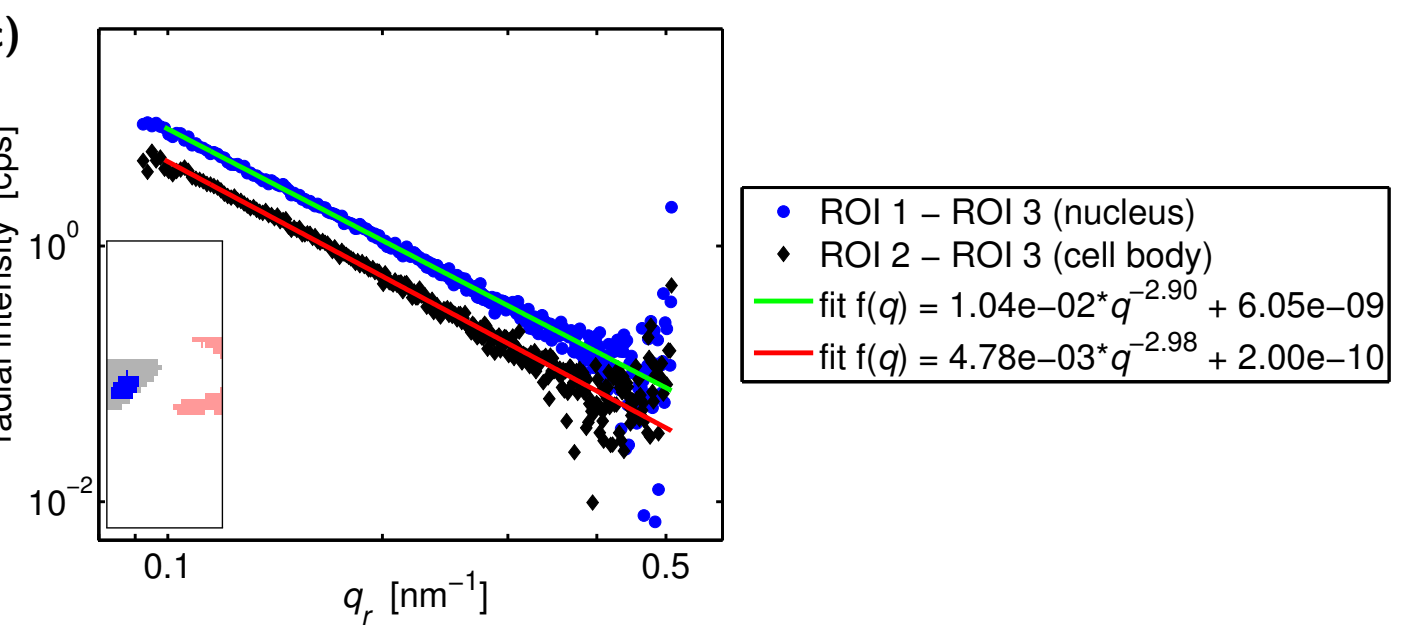

Fig. D.4.: Fitting of the background corrected average radial intensity profiles from the nucleus and the cell body with a power law. The inset shows the regions of the scan that were used for averaging of the scattering patterns (blue: nucleus, ROI 1; gray: cell body, ROI 2; red: empty, ROI 3). The $q_{r}$-ranges over which the fit functions are plotted correspond to the regions that were used for fitting. (Live Cells II.7, position 8; P10, March 2013) 

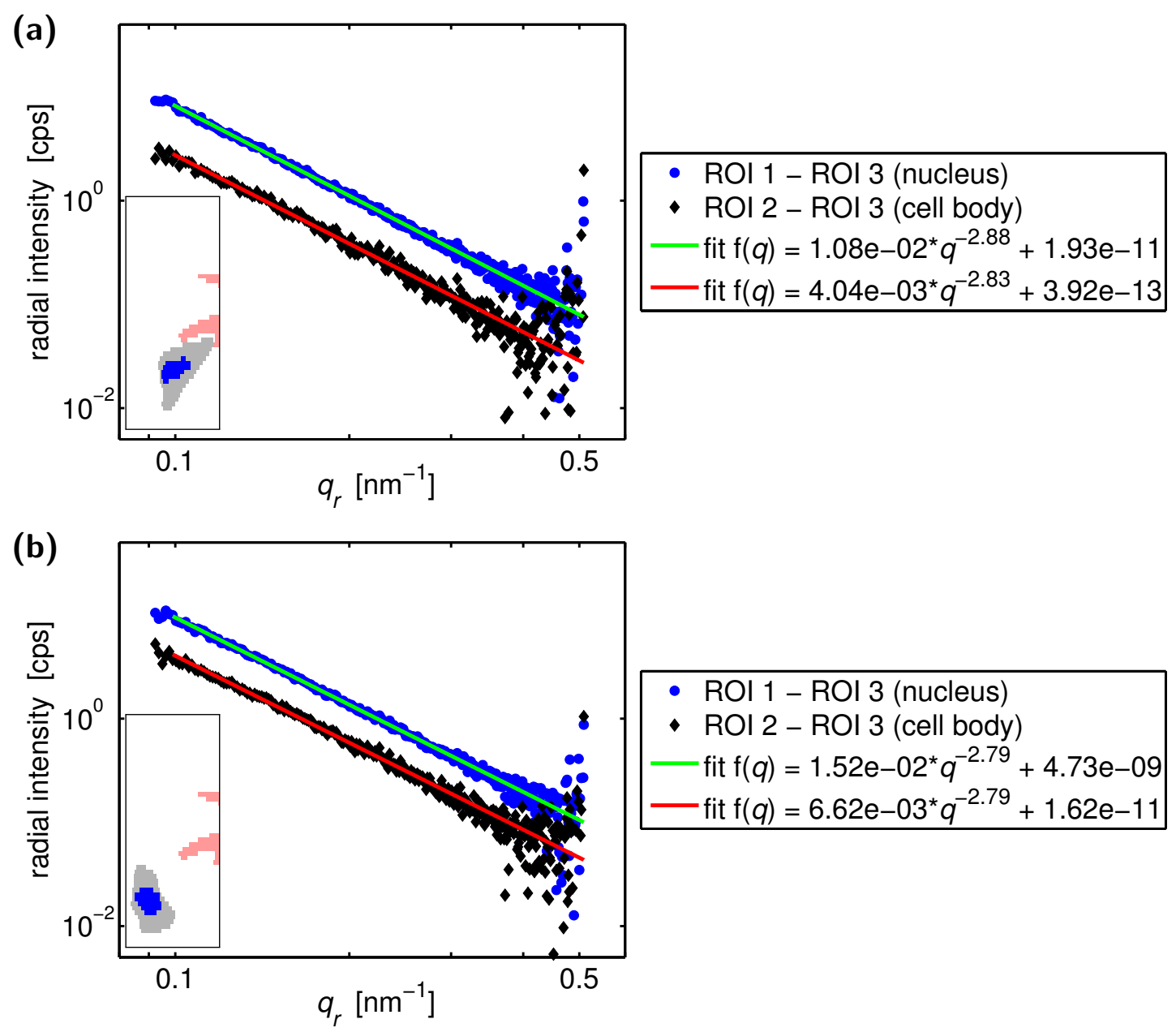

Fig. D.5.: Fitting of the background corrected average radial intensity profiles from the nucleus and the cell body with a power law. The inset shows the regions of the scan that were used for averaging of the scattering patterns (blue: nucleus, ROI 1; gray: cell body, ROI 2; red: empty, ROI 3). The $q_{r}$-ranges over which the fit functions are plotted correspond to the regions that were used for fitting. (Live Cells II.7, position 8; P10, March 2013) 


\section{E. Ptychography Measurements}

To characterize the beam at cSAXS beamline (May 2012 and Feb. 2013), ptychography measurements on a Siemens star test pattern (model ATN/XRESO-50HC, NTT-AT, Japan; consisting of a $500 \mathrm{~nm}$-thick nanostructured tantalum layer on a transparent membrane) were performed. For these measurements a slit opening of sl0wh $=0.02$ was employed. Note that different pixel sizes are obtained for the reconstructions at the two beamtimes due to different sample-to-detector distances. For the analysis of these data sets, a Matlab-based analysis, which was developed at cSAXS beamline and kindly provided, was used without further modification 95, 163.

\section{E.1. Beamtime in May 2012}

\section{E.1.1. Siemens Star Reconstruction}

The reconstructed phase and amplitude of the Siemens star test pattern are presented in Fig. E.1. The $50 \mathrm{~nm}$ lines and spaces (smallest structures) are at the resolution limit of the reconstruction.

(a)

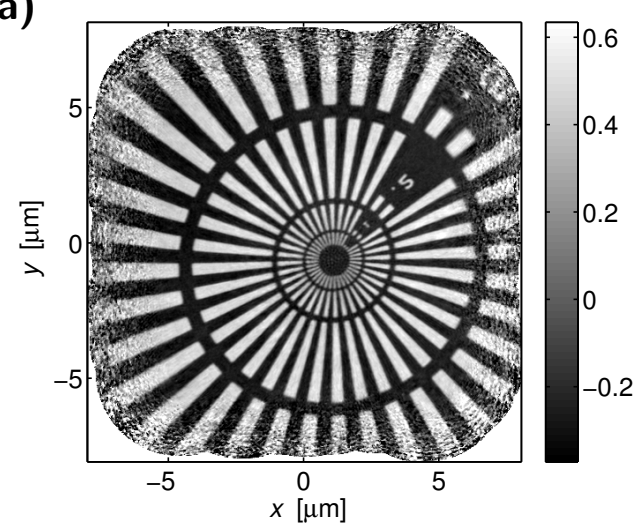

(b)

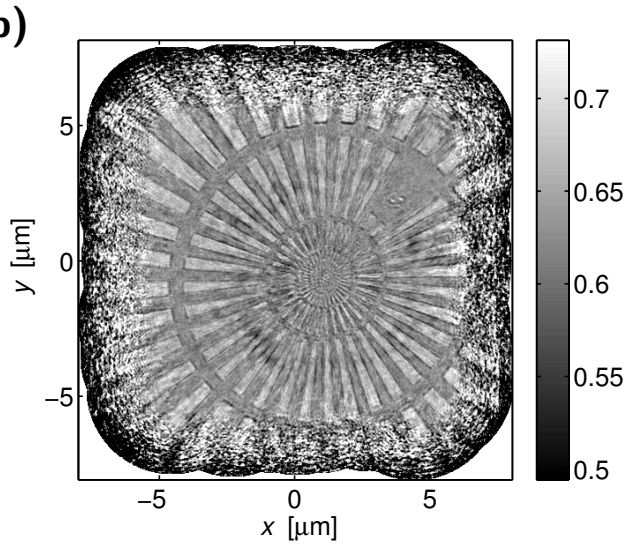

Fig. E.1.: Reconstruction of the Siemens star test pattern with a pixel size of $32.55 \mathrm{~nm}$. (a) Reconstructed phase and (b) reconstructed amplitude. (cSAXS, May 2012)

The propagated beam in the $x$-z-plane and in the $y$ - $z$-plane is depicted in Fig. E.2a and $\mathrm{b}$. Slices through the beam at $z=-1.4 \mathrm{~mm}$, i.e. where the sample was placed 
for the scanning diffraction measurements, as shown in Fig. E.2. and d. The intensity profiles were fitted with a Gaussian function to estimate the beam size. Here, a focal spot size of about $213 \times 163 \mathrm{~nm}^{2}$ (horizontal $\times$ vertical) was obtained.

(a)

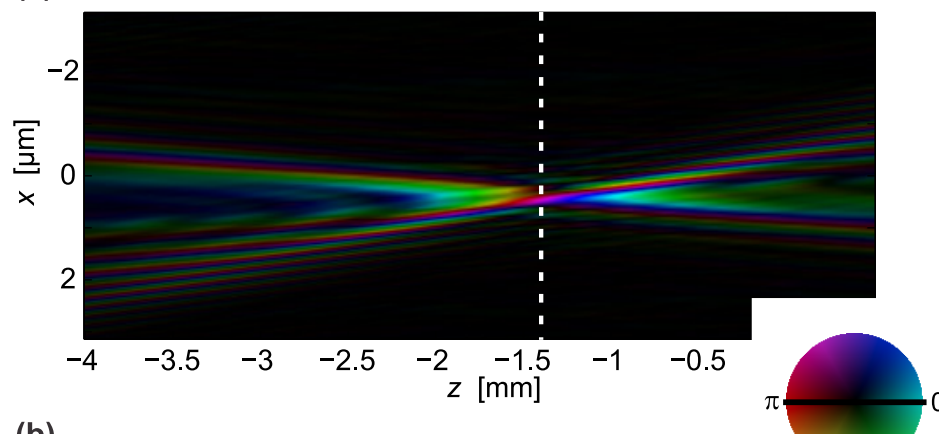

(b)

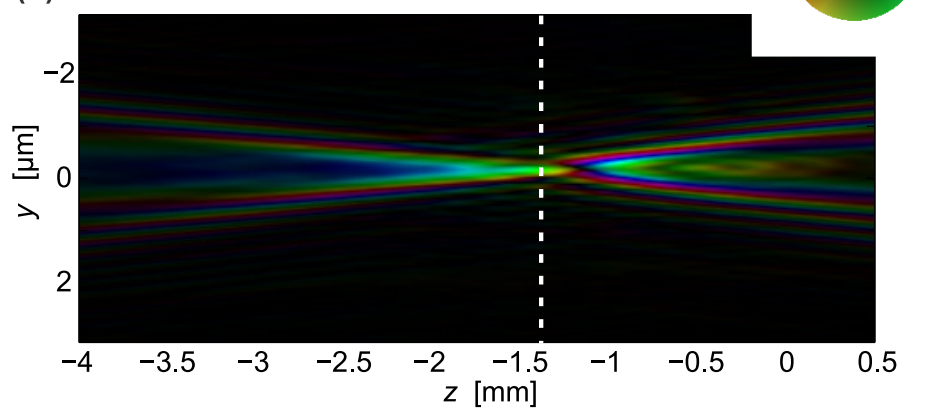

(c)

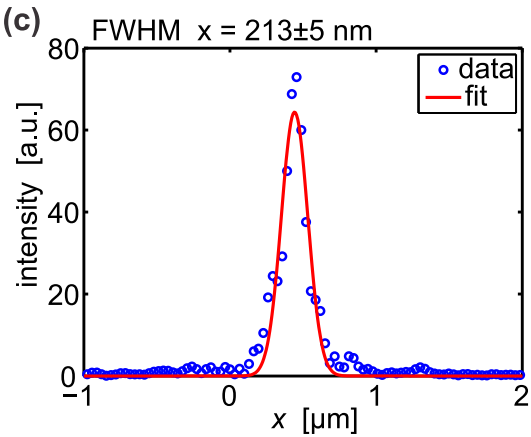

(d)

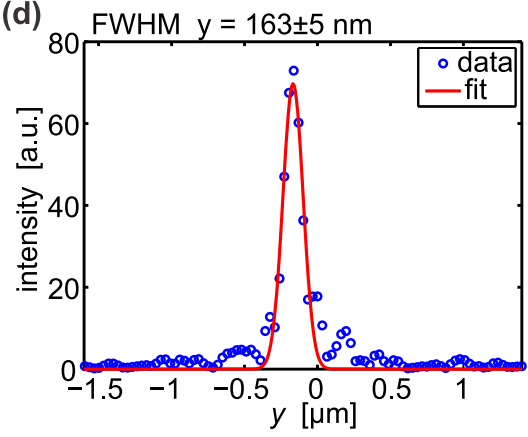

Fig. E.2.: Propagated X-ray beam (a) in the $x$-z-plane and (b) in the $y$ - $z$-plane. Fit of lateral slices (c) in $x$-direction and (d) in $y$-direction through the beam at $z=-1.4 \mathrm{~mm}$ (marked by a dashed white line in panel a and b) with a Gaussian function. The full width at half maximum is shown in the figures. (cSAXS, May 2012)

\section{E.1.2. Cell Reconstruction}

A measurement on a freeze-dried SK8/18-2 cell is presented in Fig. E.3. Fig. E.3 a shows the fluorescence microscopy image of the keratin network in a hydrated SK8/18-2 cell and Fig. E.3b shows the visible light phase contrast microscopy image of the same cell after freeze-drying. The reconstructed phase from a ptychography measurement at a defocus distance of $z=2.8 \mathrm{~mm}$ on the same cell as in Fig. E.3a, b is displayed in Fig. E.3. The cell contour can be identified and substructures are visible in the cell body as well as in the nucleus in the image showing the reconstructed phase. The positions and the shapes of the visible substructures agree well with the bright spots in and around the nucleus in the visible light phase contrast microscopy image of the freeze-dried cell. The substructures in the cell body are more densely organized close to the nucleus compared to regions in vicinity to the cell contour. This property resembles the organization of the keratin network in 
(a)

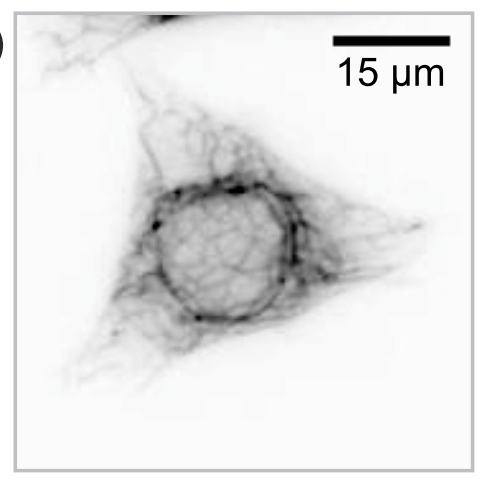

(b)

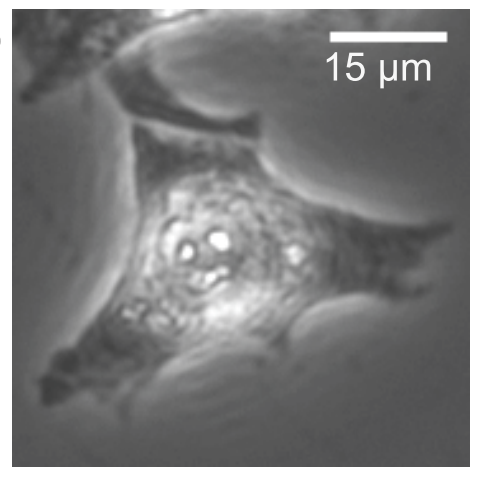

(c) 15

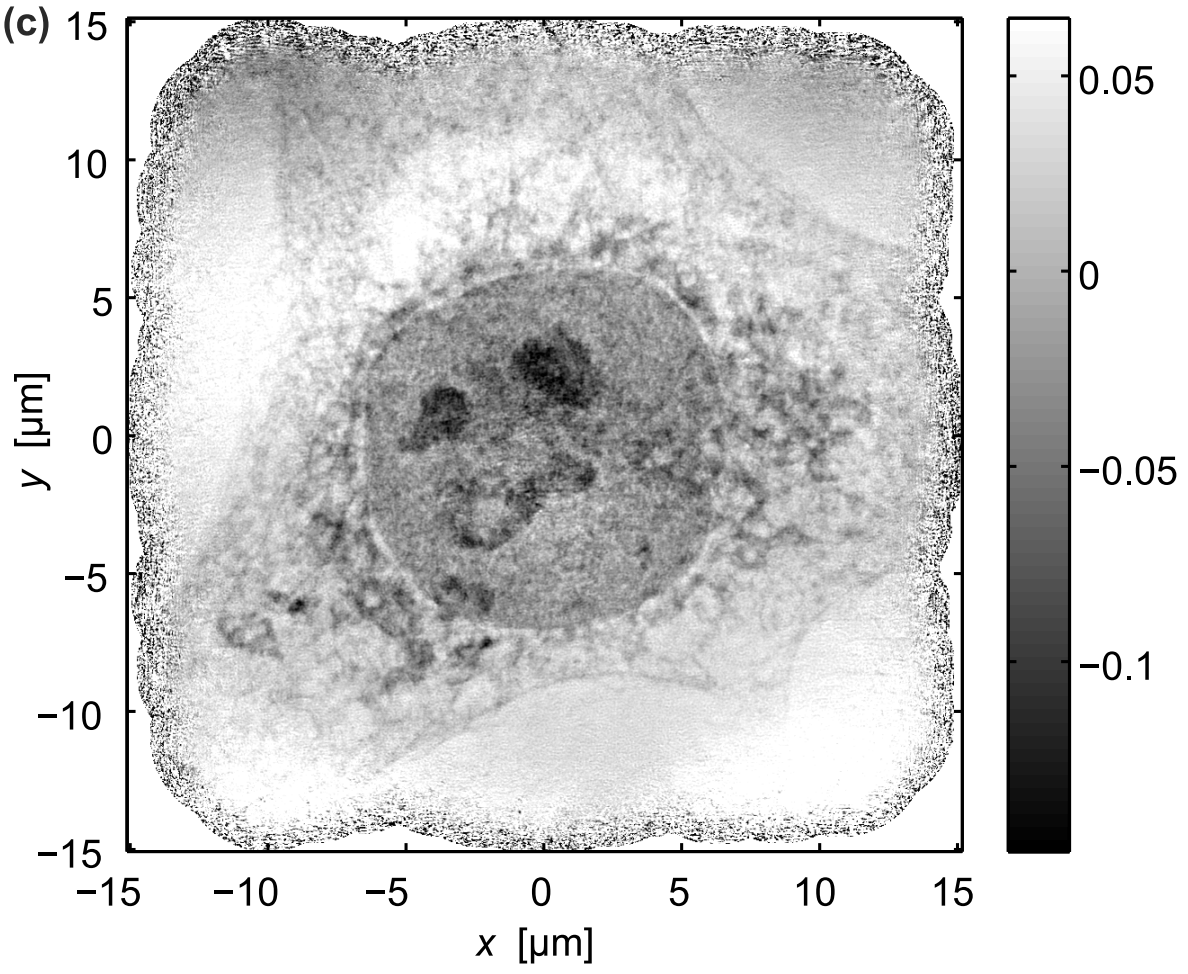

Fig. E.3.: Ptychography on a freeze-dried SK8/18-2 cell. (a) Fluorescence microscopy image of the keratin network in a hydrated SK8/18-2 cell and (b) visible light phase contrast microscopy image of the same cell after freeze-drying. (c) Reconstructed phase from a ptychography measurement at a defocus distance of $z=2.8 \mathrm{~mm}$ on the same cell as in panel a and b. (Sample BW13, position 2; cSAXS, May 2012)

SK8/18-2 cells. However, it is not possible to assign individual keratin bundles that are visible in the fluorescence microscopy image in Fig. E.3 to structures in the ptychographic reconstruction in Fig. E.3. 


\section{E.2. Beamtime in February 2013}

The reconstructed phase and amplitute of the Siemens star test pattern are presented in Fig. E.4. The $50 \mathrm{~nm}$ lines and spaces (smallest structures) can be well distinguished.

(a)

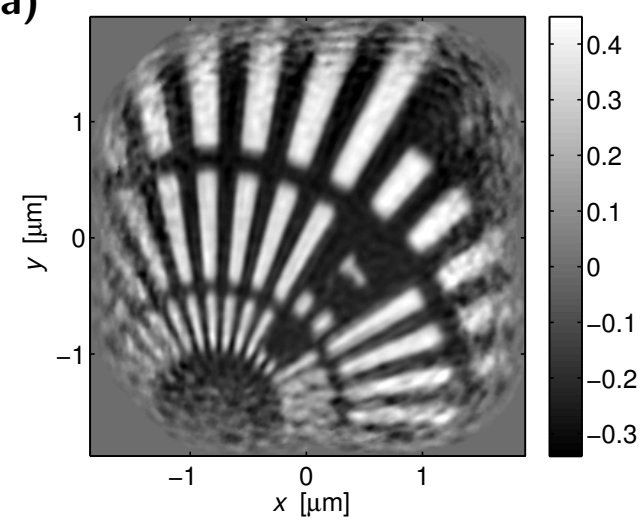

(b)

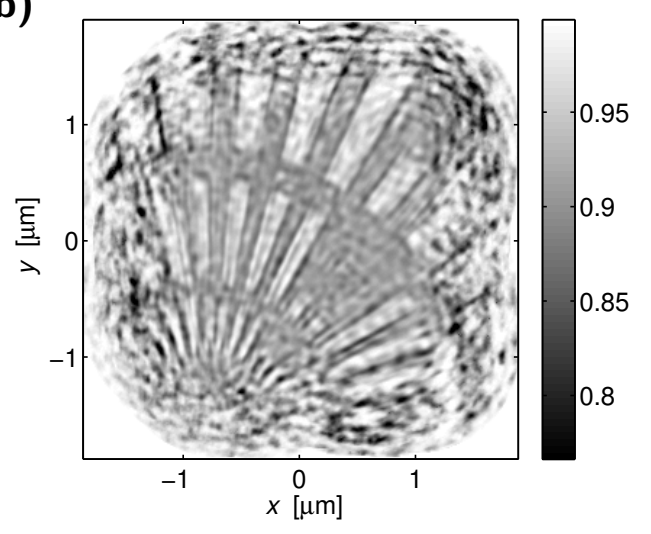

Fig. E.4.: Reconstruction of the Siemens star test pattern with a pixel size of $12.45 \mathrm{~nm}$. (a) Reconstructed phase and (b) reconstructed amplitute. (cSAXS, Feb. 2013)

The propagated beam in the $x$ - $z$-plane and in the $y$-z-plane is depicted in Fig. E.5 and $b$. For this measurements the reconstruction of the probe beam did not work well and therefore also the propagated beam is not well defined. This might be related to the shorter sample-to-detector distance. Nonetheless, this reconstruction is used to get an estimate for the beam size during the scanning diffraction measurements. Slices through the beam at $z=-0.31 \mathrm{~mm}$, i.e. where the sample was placed for the scanning diffraction measurements, as shown in Fig. E.5c and d. The intensity profiles were fitted with a Gaussian function to estimate the beam size. Here, a focal spot size of about $145 \times 223 \mathrm{~nm}^{2}$ (horizontal $\times$ vertical) was obtained, which is a reasonable value. 

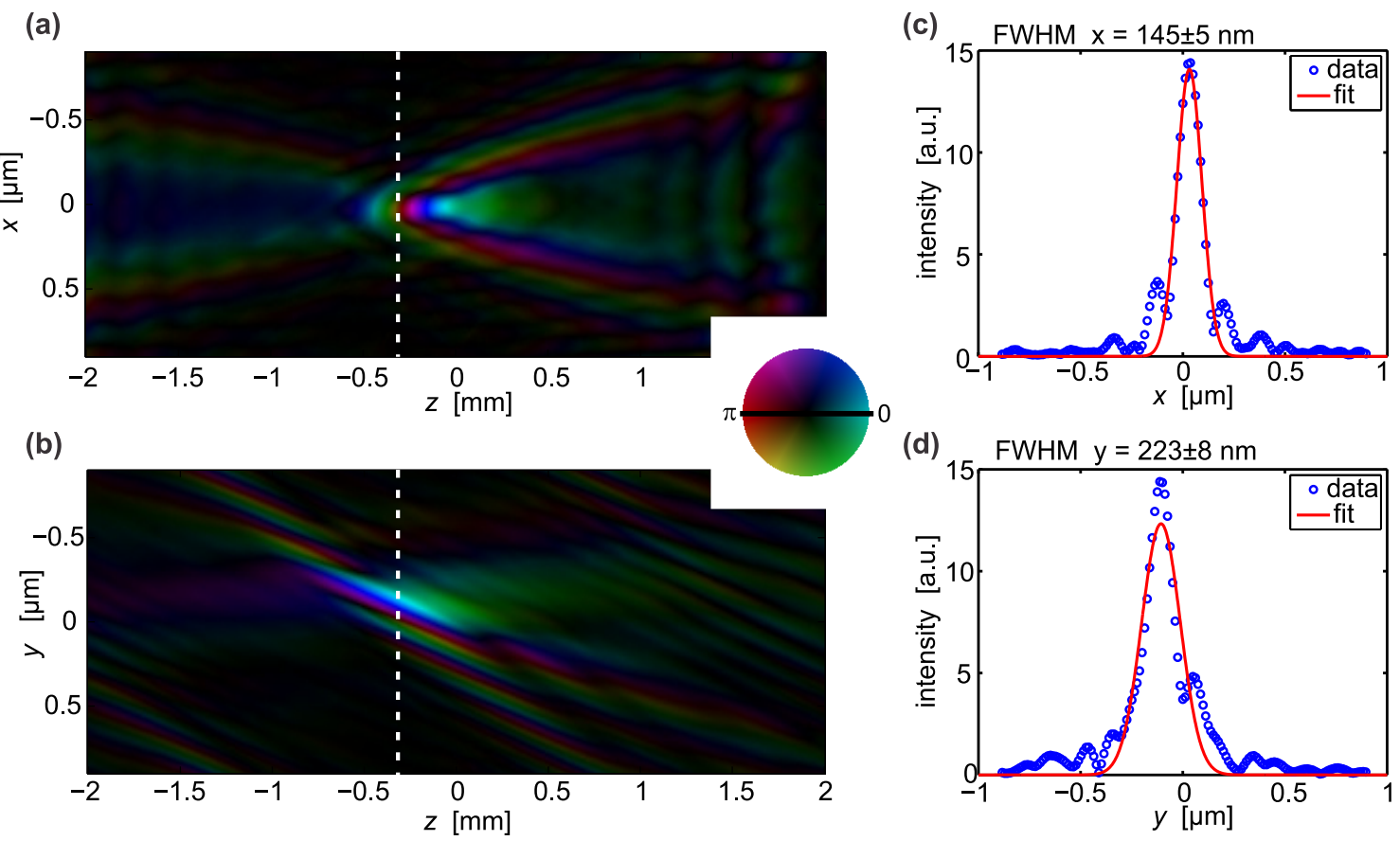

Fig. E.5.: Propagated X-ray beam (a) in the $x$-z-plane and (b) in the $y$-z-plane. Fit of lateral slices (c) in $x$-direction and (d) in $y$-direction through the beam at $z=-0.31 \mathrm{~mm}$ (marked by a dashed white line in panel a and b) with a Gaussian function. The full width at half maximum is shown in the figures. (cSAXS, Feb. 2013) 



\section{List of Abbreviations and Symbols}

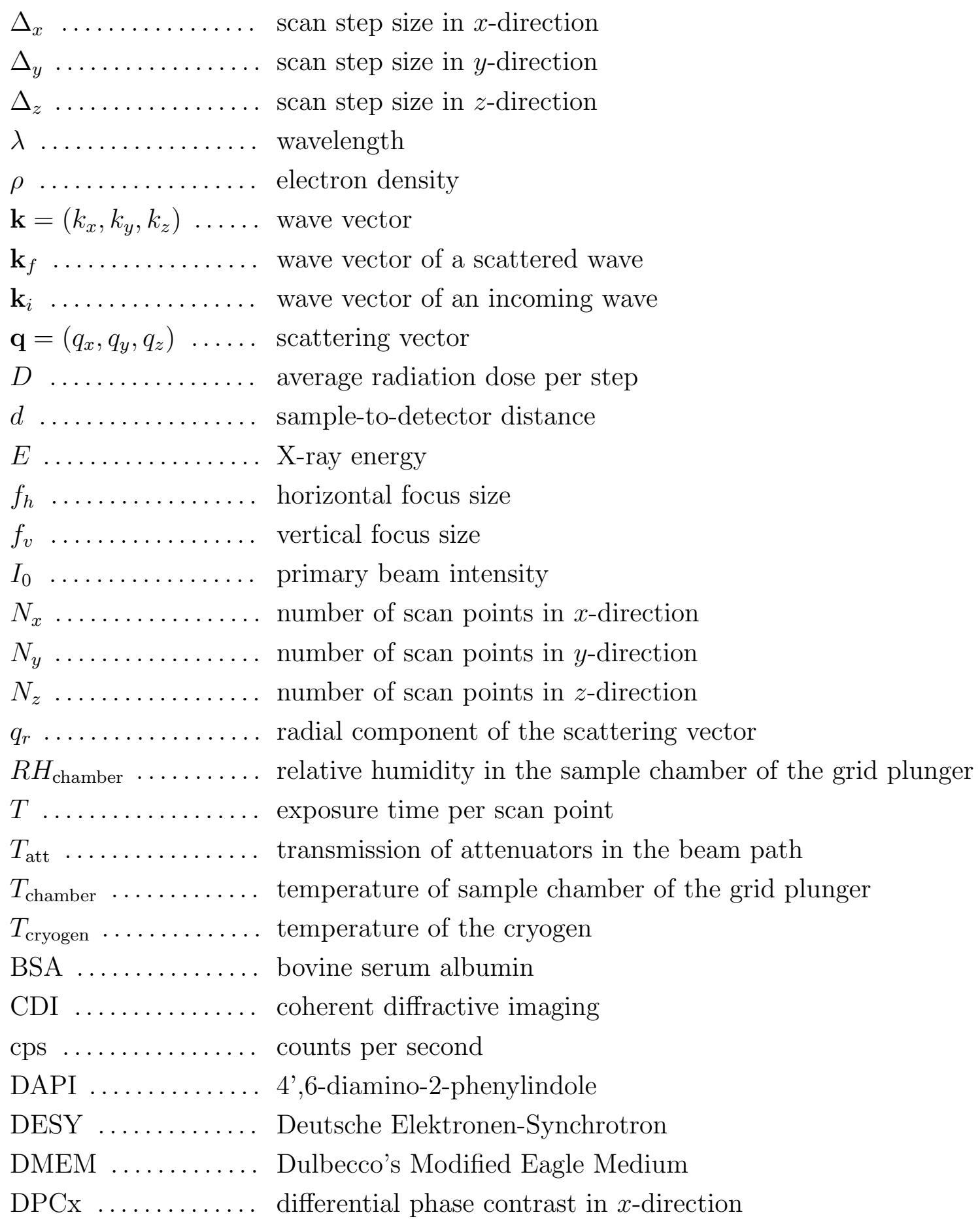


DPCy $\ldots \ldots \ldots \ldots$ differential phase contrast in $y$-direction

EDTA ............ ethylenediaminetetraacetic acid

EGTA ............ ethyleneglycoltetraacetic acid

ESRF .......... European Synchrotron Radiation Facility

F-actin .......... filamentous actin

FCS $\ldots \ldots \ldots \ldots$ fetal calf serum

FEL .............. free-electron laser

FWHM $\ldots \ldots \ldots \ldots$ full width at half maximum

IF $\ldots \ldots \ldots \ldots$ intermediate filament

KB mirrors ........ Kirkpatrick-Baez mirrors

$\mathrm{KCl} \ldots \ldots \ldots \ldots$ potassium chloride

KIF ........... keratin intermediate filament

MFs ........... mircofilaments, actin filaments

MTs ............. microtubules

$\mathrm{NaN}_{3} \ldots \ldots \ldots . . . .$. sodium azide

NEXAFS ......... near edge X-ray absorption fine structure

PBS $\ldots \ldots \ldots \ldots \ldots$ phosphate buffered saline

PBS/Pen-Strep ..... PBS supplemented with $100 \mathrm{U} / \mathrm{ml}$ penicillin and $0.1 \mathrm{mg} / \mathrm{ml}$ streptomycin

PDMS ........... polydimethylsiloxane

Pen-Strep ........ penicillin-streptomycin $(100 \mathrm{U} / \mathrm{ml}$ penicillin and $0.1 \mathrm{mg} / \mathrm{ml}$ streptomycin)

PETRA III ........ Positron-Elektron-Tandem-Ring-Anlage III

PMSF ........... phenylmethylsulfonyl fluoride

PSI ............. Paul Scherrer Institut

SAXS $\ldots \ldots \ldots \ldots$ small-angle X-ray scattering

SEM $\ldots \ldots \ldots \ldots \ldots$ scanning electron microscopy

$\mathrm{Si}_{3} \mathrm{~N}_{4} \ldots \ldots \ldots \ldots$ silicon nitride

SLS ............ Swiss Light Source

STED ............. stimulated emission depletion

TAME $\ldots \ldots \ldots \ldots . \mathrm{N} \alpha$-p-Tosyl-L-arginine methyl ester hydrochloride 


\section{List of Figures}

2.1. Fluorescence microscopy image of a cell. . . . . . . . . . . . . . . 6

2.2. Schematic model of the KIF assembly. . . . . . . . . . . . . . . . . 8

2.3. Schematic of the keratin cycle. . . . . . . . . . . . . . . . . 9

2.4. Fluorescence microscopy image and SEM image of the keratin net-

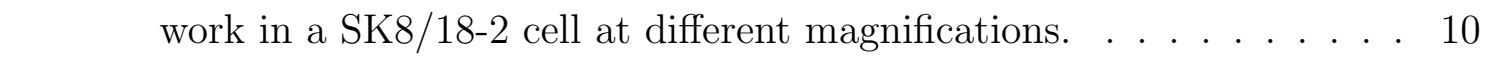

2.5. Cryo transmission electron micrograph of a vitreous section of the stratum corneum. . . . . . . . . . . . . . . . . . . . 11

2.6. Actin filament bundle in hair cells. . . . . . . . . . . . . . . . . . 13

3.1. Schematic of the geometry assumed for the derivation of the projec-

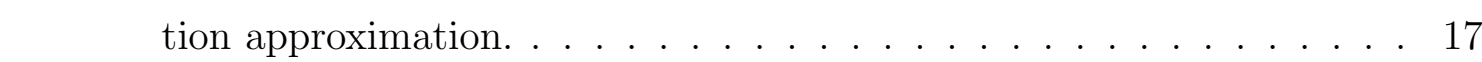

3.2. Relationship between the deflection angle $\alpha$ and the real part of the

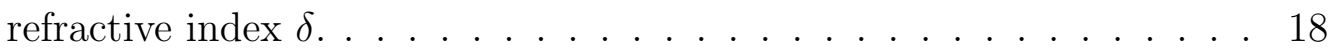

3.3. Sketch of the set-up at the experimental hutch III at the ID13 beamline. 23

3.4. Schematic of the measurement set-up at the P10 beamline. . . . . . . 24

3.5. Schematic of the measurement set-up at the cSAXS beamline. . . . . 25

4.1. Schematic of the preparation pathway of different sample types for synchrotron experiments. . . . . . . . . . . . . . . . . . 31

4.2. Photographs of the grid plunger in the different positions during plunge-freezing. . . . . . . . . . . . . . . 34

4.3. Photographs of the sample at different steps during plunge-freezing. . 35

4.4. Microscopy images of a hydrated sample and the same sample after freeze-drying. . . . . . . . . . . . . . . . . 36

4.5. Fluorescence staining of F-actin and DNA in native SK8/18-2 cells and in SK8/18-2 cells treated with IF lysis buffer. . . . . . . . . . . . 38

4.6. Fluorescence staining of native SK8/18-2 cells and SK8/18-2 cells treated with IF lysis buffer (microtubules, keratin, DNA). . . . . . . . 39

4.7. Bright-field microscopy image of SK8/18-2 cell treated with IF lysis buffer. . . . . . . . . . . . . . . . . . . 4 40

4.8. 3D schematic and photographs of a microfluidic device. . . . . . . . . 42

4.9. $\mathrm{Si}_{3} \mathrm{~N}_{4}$ sandwich wet chambers. . . . . . . . . . . . . . . . 42 
4.10. Schematic of the fabrication process of X-ray compatible microfluidic devices. . . . . . . . . . . . . . . . . . 44 44

4.11. Photographs of the device fabrication process. . . . . . . . . . . 45

4.12. Photographs of the devices assembly process. . . . . . . . . . . . . . 47

5.1. $\mathrm{Si}_{3} \mathrm{~N}_{4}$ membrane window mounted on a crocodile sample holder. . . . 49

5.2. X-ray diffraction from highly aligned keratin bundles in freeze-dried cells. (Sample BW17, position 1; ID13, June 2011) . . . . . . . . . . 50

5.3. Image processing for the determination of the signal anisotropy. (Sample BW17, position 1; ID13, June 2011) . . . . . . . . . . . . . . 54

5.4. Structure orientation in the cellular extension of a freeze-dried

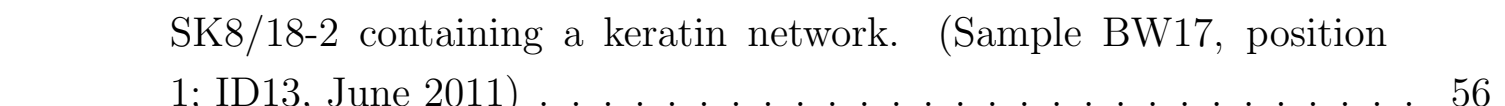

5.5. Structure orientation map and dark-field image of the cell body of a

\begin{tabular}{|c|}
\hline freeze-dried SK8/18-2 cell. (Sample BW21, position 4; ID13, Nov. \\
\hline
\end{tabular}

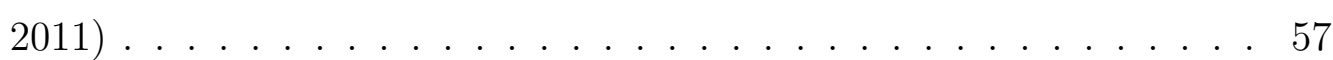

5.6. Azimuthal integration of averaged scattering patterns in eight angular segments for a cell containing a keratin network. (Sample BW17, position 1; ID13, June 2011) . . . . . . . . . . . . . . . . . . . . . 59

5.7. Fitting of the radial intensity with a power law for a cell containing a keratin network. (Sample BW17, position 1; ID13, June 2011) . . . 60

5.8. Azimuthal integration of a single scattering pattern and an average of $2 \times 2$ scattering patterns in eight angular segments for a cell containing a keratin network. (Sample BW17, position 1; ID13, June 2011)] . . . 61

5.9. Fitting of the average the first and fifth radial intensity profile of a single scattering pattern. (Sample BW17, position 1; ID13, June 2011) 62

5.10. Microscopy images and coarse X-ray dark-field image of a group of

\begin{tabular}{|c|}
\hline freeze-dried SK8/18-2 cells. (Sample BW21, position 6; ID13, Nov. \\
\hline
\end{tabular}

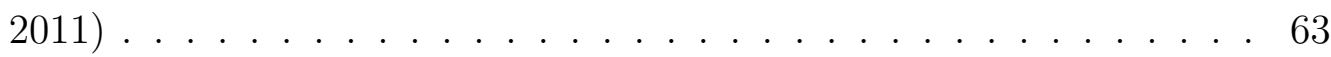

5.11. Structure orientation and X-ray dark-field image of the cellular extensions of a keratin-free freeze-dried SK8/18-2 cell. (Sample BW21, position 6; ID13, Nov. 2011) . . . . . . . . . . . . . . . . . . . . . . 64

5.12. Azimuthal integration of averaged scattering patterns for a keratinfree cell. (Sample BW21, position 6; ID13, Nov. 2011) . . . . . . . . 66

5.13. X-ray dark-field image and structure orientation in freeze-dried cells that was treated with IF lysis buffer. (Sample BW54, position 1; ID13, June 2011) . . . . . . . . . . . . . . . . . . . . . . 67 
5.14. Azimuthal integration of averaged scattering patterns for a cell that

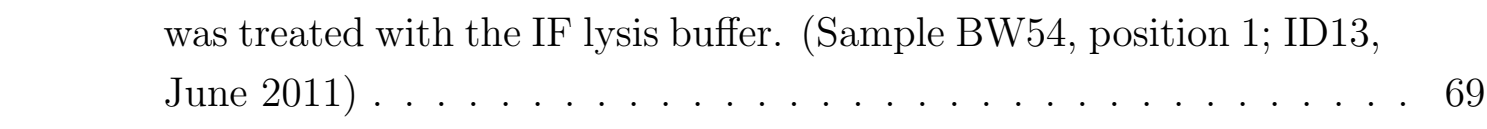

5.15. Microscopy images and X-ray dark-field image of a freeze-dried SK8/18-2 cell with a keratin network. (Sample BW129, position 3; P10, Oct. 2012) . . . . . . . . . . . . . . . 71

5.16. Maps of the direction and degree of structure orientation in the sample. (Sample BW129, position 3; P10, Oct. 2012) . . . . . . . . . . 72

5.17. Overlay of the X-ray dark-field image and the vector field combin-

ing the direction and the degree of local structure orientation in the
sample. (Sample BW129, position 3; P10, Oct. 2012) . . . . . . . . . 73

5.18. Averaged scattering patterns from three different scan regions. (Sample BW129, position 3; P10, Oct. 2012) . . . . . . . . . . . . . . . 74

5.19. Radial intensity profiles of averaged scattering patterns. (Sample BW129, position 3; P10, Oct. 2012) . . . . . . . . . . . . . 75

5.20. Fitting of two power laws to the low $q_{r}$-region and high $q_{r}$-region of the background corrected average radial intensity profiles. (Sample BW129, position 3; P10, Oct. 2012) . . . . . . . . . . . . . 75

5.21. Comparison of the power law exponents for freeze-dried cells with and without a keratin network measured at the P10 beamline. . . . . . . . 76

5.22. Single scattering pattern from four different scan positions. (Sample

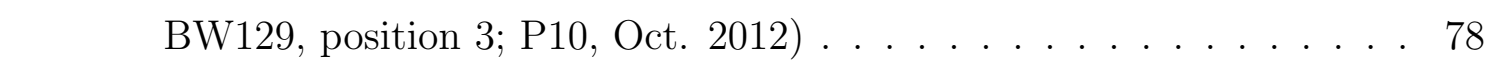

5.23. Fitting of two power laws to the low $q_{r}$-region and high $q_{r}$-region of \begin{tabular}{|c|}
\hline the background corrected radial intensity profiles of single scattering \\
\hline
\end{tabular} patterns (Sample BW129, position 3; P10, Oct. 2012) . . . . . . . . . 79

5.24. Azimuthal integration of a scattering pattern from the cellular extension in eight angular segments. (Sample BW129, position 3; P10, Oct. 2012) . . . . . . . . . . . . . . . . . . 8 81

5.25. Microscopy images and X-ray dark-field image of freeze-dried SK8/182 cells. (Sample BW203, position 2; cSAXS, Feb. 2013) . . . . . . . . 83

5.26. Structure orientation map as overlay with the X-ray dark-field image. (Sample BW203, position 2B; cSAXS, Feb. 2013) . . . . . . . . . . 83

5.27. Azimuthal integration of averaged scattering patterns. (Sample

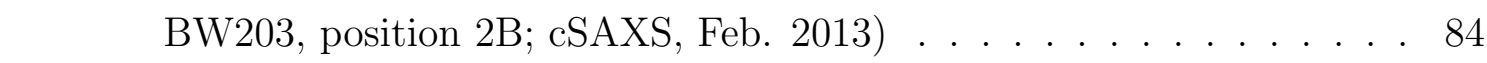

5.28. Fitting of two power laws to the low $q_{r}$-region and high $q_{r}$-region of patterns (Sample BW203, position 2B; cSAXS, Feb. 2013) . . . . . . 85 
5.29. Azimuthal integration of a scattering pattern from the cellular exten-

\begin{tabular}{|l}
\hline sion in eight angular segments. (Sample BW203, position 2B; cSAXS, \\
\hline \hline Feb. 2013) . . . . . . . . . . . . . . . . . . 86
\end{tabular}

5.30. Azimuthal integration of an average of $3 \times 3$ scattering patterns from

\begin{tabular}{|c|}
\hline the cellular extension in eight angular segments. (Sample BW203, \\
\hline
\end{tabular}
position 2B; cSAXS, Feb. 2013) . . . . . . . . . . . . . 87

5.31. Comparison of radial intensity profiles of averaged scattering patterns

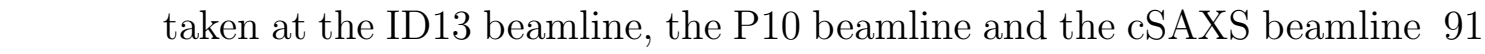

5.32. Summary of all power law exponents obtained from fits to the radial \begin{tabular}{|c|}
\hline intensity profiles of averaged scattering patterns recorded on freeze- \\
\hline
\end{tabular} ․ dried SK8/18-2 cells. . . . . . . . . . . . . . . . . 92

6.1. Photograph of a $\mathrm{Si}_{3} \mathrm{~N}_{4}$ sandwich wet chamber mounted on a magnetic

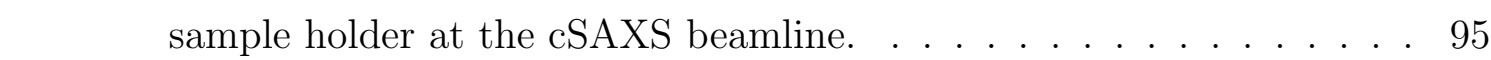

6.2. Photograph of a microfluidic device mounted on a magnetic sample holder at the P10 beamline. . . . . . . . . . . . . . . . . . 96

6.3. Microscopy images and different X-ray contrast images of fixed\begin{tabular}{|c|}
\hline hydrated SK8/18-2 cells with a keratin network. (Sample BW108, \\
\hline
\end{tabular}

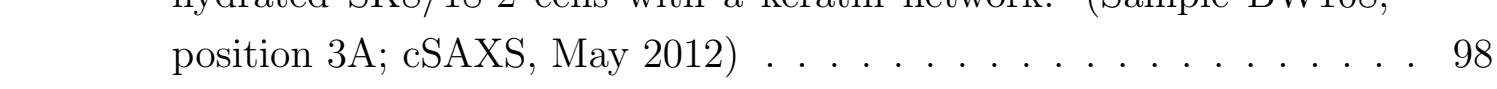

6.4. Region of interest measurements on fixed-hydrated SK8/18-2 cells \begin{tabular}{|c|}
\hline with a keratin network. (Sample BW108, position 3; cSAXS, May \\
\hline
\end{tabular} 2012) . . . . . . . . . . . . . . . . . . 99

6.5. Microscopy images and DPCx image of a fixed-hydrated SK8/18-2 cell without a keratin network. (Sample BW108, position 4A; cSAXS, May 2012) . . . . . . . . . . . . . . . . . . . . 100

6.6. Region of interest measurement on a fixed-hydrated SK8/18-2 cell without a keratin network. (Sample BW108, position 4; cSAXS, May 2012) . . . . . . . . . . . . . . . . . . . . . . . . . . . . . . . . . .

6.7. Averaged scattering patterns from three different scan regions. (Sample BW108, position 3A; cSAXS, May 2012) . . . . . . . . . . . . . . 101

6.8. Radial intensity profiles from averaged scattering patterns. (Sample BW108, position 3A; cSAXS, May 2012) . . . . . . . . . . . . . . 102

6.9. Detector mask used for the calculation of the X-ray dark-field images of hydrated samples measured at the ID13 beamline. . . . . . . . . . 104

6.10. Microscopy images and X-ray dark-field image of a fixed-hydrated

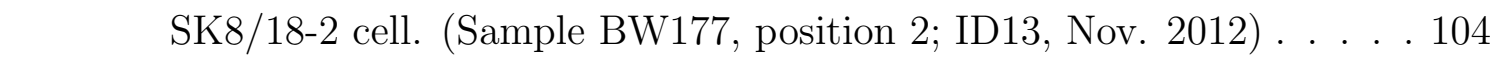

6.11. Averaged scattering pattern from three different scan regions on a

\begin{tabular}{|c|}
\hline fixed-hydrated SK8/18-2 cell. (Sample BW177, position 2; ID13, \\
\hline \hline
\end{tabular}
Nov. 2012). . . . . . . . . . . . . . . . . 105 
6.27. Detachment of a fixed-hydrated SK8/18-2 cell from the substrate dur-

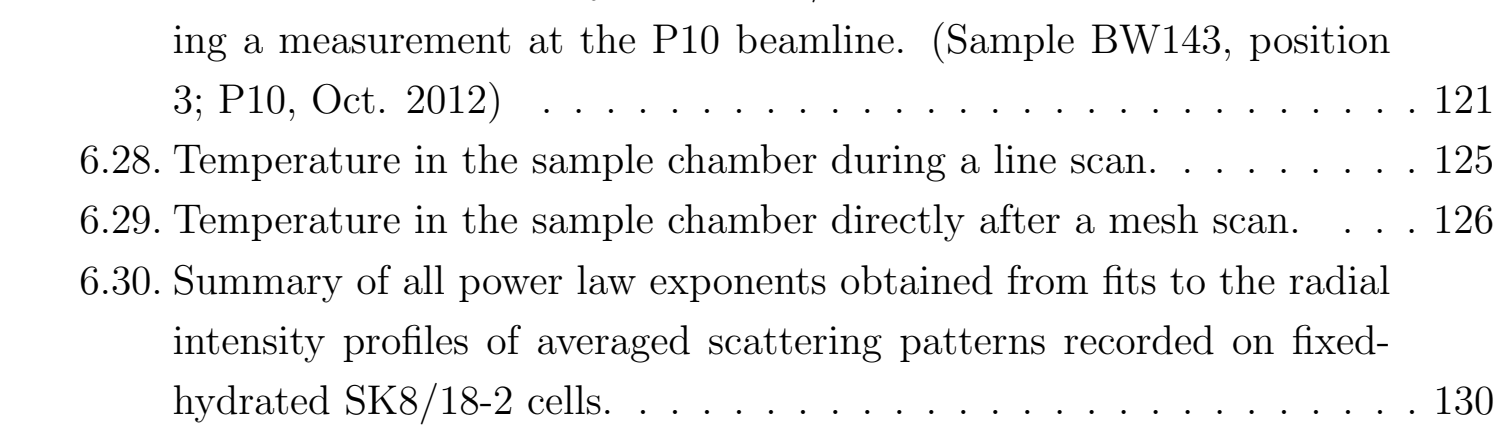

7.1. Bright-field microscopy image and X-ray dark-field image of (initially)

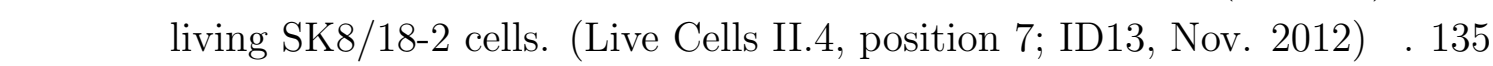

7.2. Visible light microscopy images and X-ray dark-field image of living SK8/18-2 cells. (Live Cells II.7, position 7; P10, March 2013)] . . . . . 138

7.3. Averaged scattering patterns from three different scan regions. (Live Cells II.7, position 7; P10, March 2013) . . . . . . . . . . . . . . . . . 139

7.4. Radial intensity profiles of averaged scattering patterns. (Live Cells

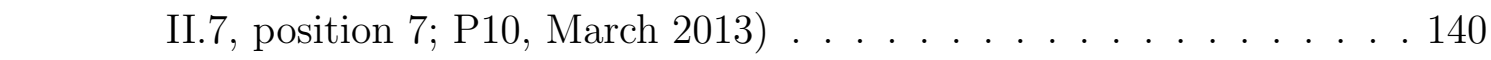

7.5. Fitting of a power law to the background corrected average radial

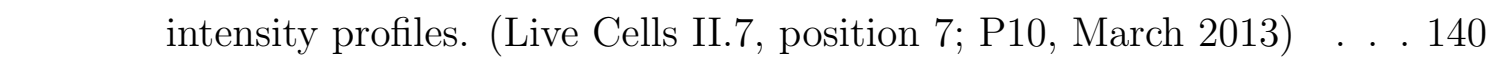

7.6. Comparison of the power law exponents for living cells. (P10, March 2013) . . . . . . . . . . . . . . . . . . 141

7.7. Radiation damage to living SK8/18-2 cells during a measurement at the P10 beamline. (Sample BW143, position 3; P10, Oct. 2013) . . . 143

8.1. Comparison of power law exponents obtained from fits to the back-

\begin{tabular}{|c|}
\hline ground corrected radial intensity profiles for freeze-dried, fixed- \\
\hline
\end{tabular}
hydrated and living SK8/18-2 cells. . . . . . . . . . . . . . . . . . 148

8.2. Averaged radial intensity profiles for living, fixed-hydrated and freeze-

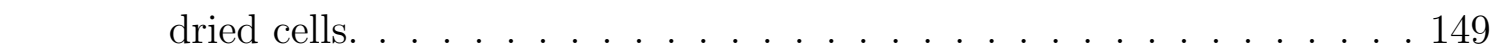

8.3. Difference of the averaged radial intensity profiles for living and fixed- hydrated cells. . . . . . . . . . . . . . . . . 149

B.1. Microscopy images and X-ray dark-field image of a group of SK8/18-2 cells. (Sample BW130, position 1; P10, Oct. 2012) . . . . . . . . . . 159

B.2. Fitting of the low $q_{r}$-region and high $q_{r}$-region of the background \begin{tabular}{|l|}
\hline corrected average radial intensity profiles from the nucleus and the \\
\hline cell body with power laws. (Sample BW130, position 1; P10, Oct. \\
\hline \hline 2012$)$
\end{tabular}

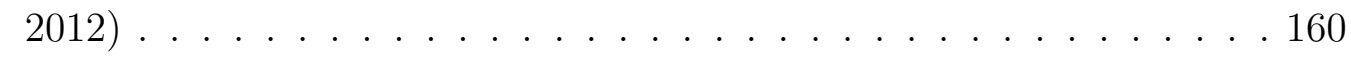

B.3. Microscopy images and X-ray dark-field image. (Sample BW130, pos 2; P10, Oct. 2012) . . . . . . . . . . . . . . 161 
C.8. Microscopy images and X-ray dark-field image of fixed-hydrated SK8/18-2 cells. (Sample BW145, position 3; P10, Oct. 2012)] . . . . . 171

C.9. Microscopy images and X-ray dark-field image of fixed-hydrated SK8/18-2 cells. (Sample BW145, position 4; P10, Oct. 2012)] . . . . . 171

C.10.Fitting of the low $q_{r}$-region and high $q_{r}$-region of the background corrected average radial intensity profiles from the nucleus and the cell body with power laws. (Sample BW145, position 4; P10, Oct. 2012) . . . . . . . . . . . . . . . . . . . 172

C.11.Microscopy images and X-ray dark-field image of fixed-hydrated

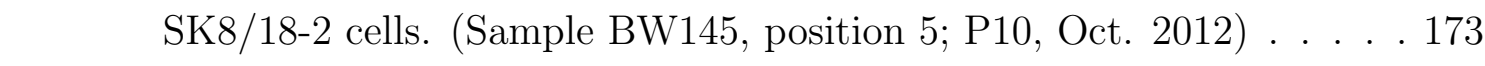

C.12.Fitting of the low $q_{r}$-region and high $q_{r}$-region of the background corrected average radial intensity profiles from the nucleus and the cell body with power laws. (Sample BW145, position 5; P10, Oct.

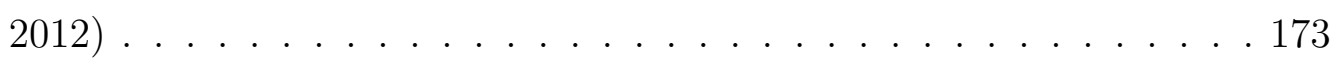

C.13.Fitting of the low $q_{r}$-region and high $q_{r}$-region of the background corrected average radial intensity profiles from the nucleus and the cell body with power laws. (Sample BW145, position 5; P10, Oct. 2012) . . . . . . . . . . . . . . . . . 174

C.14.Microscopy images and X-ray dark-field image of fixed-hydrated SK8/18-2 cells. (Sample BW156, position 3; P10, Oct. 2012)] . . . . . 175

C.15.Fitting of the low $q_{r}$-region and high $q_{r}$-region of the background corrected average radial intensity profiles from the nucleus and the cell body with power laws. (Sample BW156, position 3; P10, Oct. 2012).

C.16.Fitting of the low $q_{r}$-region and high $q_{r}$-region of the background corrected average radial intensity profiles from the nucleus and the cell body with power laws. (Sample BW235, position 1; P10, March 2013) . . . . . . . . . . . . . . . 176

C.17.Microscopy images and X-ray dark-field image of fixed-hydrated SK8/18-2 cells. (Sample BW237, position 1; P10, March 2013)] . . . . 176

C.18.Fitting of the low $q_{r}$-region and high $q_{r}$-region of the background corrected average radial intensity profiles from the nucleus and the cell body with power laws. (Sample BW237, position 1; P10, March

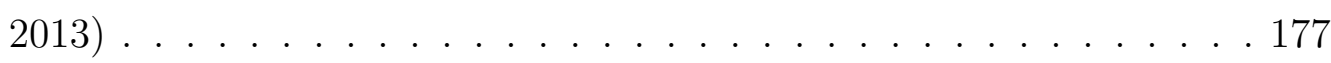

C.19.Microscopy images and X-ray dark-field image of fixed-hydrated SK8/18-2 cells. (Sample BW237, position 2; P10, March 2013)] . . . . 178 
C.20.Fitting of the low $q_{r}$-region and high $q_{r}$-region of the background

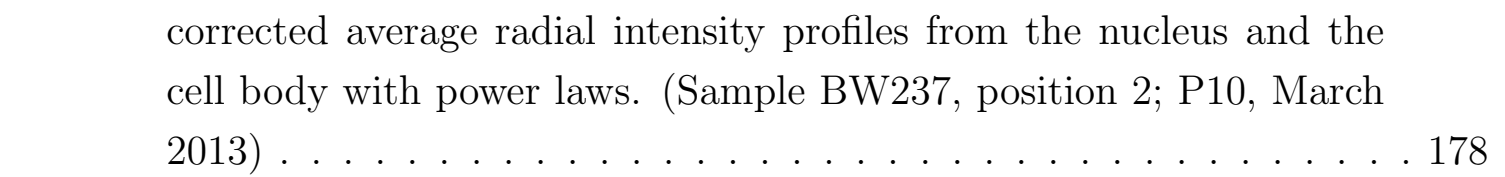

C.21.Microscopy images and X-ray dark-field image of fixed-hydrated SK8/18-2 cells. (Sample BW237, position 3; P10, March 2013)] . . . . 179

C.22.Fitting of the low $q_{r}$-region and high $q_{r}$-region of the background corrected average radial intensity profiles from the nucleus and the cell body with power laws. (Sample BW237, position 3; P10, March 2013) . . . . . . . . . . . . . . . . . . . . 179

D.1. Fitting of the background corrected average radial intensity profiles from the nucleus and the cell body with a power law. (Live Cells II.7, position 7; P10, March 2013)] . . . . . . . . . . . . . . . . . . . . . . 181

D.2. Visible-light microscopy and X-ray dark-field image. (Live Cells II.7, position 8; P10, March 2013). . . . . . . . . . . . . . . . . . . . . 182

D.3. Fitting of the background corrected average radial intensity profiles from the nucleus and the cell body with a power law. (Live Cells II.7, position 8; P10, March 2013) . . . . . . . . . . . . . . . . . . . 182

D.4. Fitting of the background corrected average radial intensity profiles from the nucleus and the cell body with a power law. (Live Cells II.7, position 8; P10, March 2013) . . . . . . . . . . . . . . . . 183

D.5. Fitting of the background corrected average radial intensity profiles from the nucleus and the cell body with a power law. (Live Cells II.7, position 8; P10, March 2013). . . . . . . . . . . . . . . . . . . 184

E.1. Reconstruction of the Siemens star test pattern. (cSAXS, May 2012) 185 E.2. Propagated X-ray beam. (cSAXS, May 2012) . . . . . . . . . . . . . 186

E.3. Ptychography on a freeze-dried SK8/18-2 cell. (Sample BW13, position 2; cSAXS, May 2012) . . . . . . . . . . . . . . . . . . 187

E.4. Reconstruction of the Siemens star test pattern. (cSAXS, Feb. 2013) 188

E.5. Propagated X-ray beam. (cSAXS, Feb. 2013) . . . . . . . . . . . . . 189 



\section{List of Tables}

2.1. Overview of the six different IF types and their tissue distribution. . . 7

3.1. List of set-up parameters during the different beamtimes. . . . . . . . 22

4.1. Boiling and melting points of ethane, propane, nitrogen and a mixture of $37 \%$ ethane and $63 \%$ propane. . . . . . . . . . . . . . 33

4.2. Set-points during plunge-freezing with the Leica grid plunger . . . . . 33

4.3. Recipe of IF lysis buffer that was used for IF preparation. . . . . . . 37

5.1. Summary of the scan parameters and the estimated average radiation doses per step for the measurements on freeze-dried SK8/18-2 cells at the ID13 beamline. . . . . . . . . . . . . . . . . . . . . . 52

5.2. Summary of the scan parameters and the estimated average radiation doses per step for the measurements on freeze-dried cells at the P10 beamline. ......................... 70

5.3. Mean power law exponents and standard deviation of the mean for all \begin{tabular}{|l|l|}
\hline freeze-dried SK8/18-2 cells that were measured at the P10 beamline. \\
\hline \hline (P10, Oct. 2012 and March 2013) . . . . . . . . . . . . 77
\end{tabular}

5.4. Summary of the scan parameters and the estimated average radiation \begin{tabular}{|l}
\hline doses per step for the measurements on freeze-dried cells at the cSAXS \\
\hline beamline. . . . . . . . . . . . . . . . . . . . . . . . . . .
\end{tabular}

6.1. Summary of the scan parameters and the estimated average radiation \begin{tabular}{|c|}
\hline doses per step for the measurements on fixed-hydrated cells at the \\
\hline
\end{tabular} cSAXS beamline. . . . . . . . . . . . . . . . . . 97

6.2. Summary of the scan parameters and the estimated average radiation doses per step for the measurements on fixed-hydrated cells at the ID13 beamline. . . . . . . . . . . . . . . . . 103

6.3. Summary of the scan parameters and the estimated average radiation

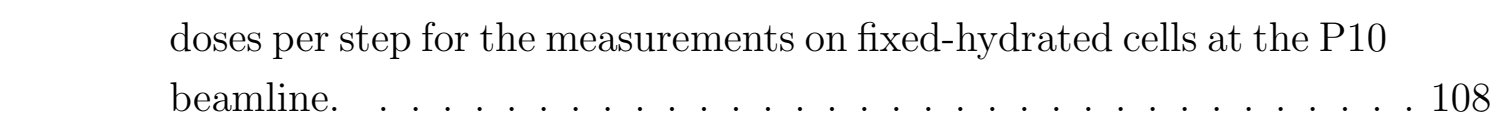


6.4. Mean power law exponents and standard deviation of the mean for all fixed-hydrated SK8/18-2 cells measured at the P10 beamline. (P10, Oct. 2012 and March 2013) . . . . . . . . . . . . . . . . . . . . . . 114

7.1. Summary of the scan parameters and the estimated average radiation dose per step for the measurement on living cells at the ID13 beamline. 135

7.2. Summary of the scan parameters and the estimated average radiation doses per step for the measurements on living cells at the P10 beamline.137

7.3. Mean power law exponents and standard deviation of the mean for all (initially) living SK8/18-2 cells measured at the P10 beamline. (P10, Oct. 2012 and March 2013) . . . . . . . . . . . . . . . . . . . . . . . 141 


\section{Bibliography}

[1] Fuchs, E. \& Cleveland, D. W. A Structural Scaffolding of Intermediate Filaments in Health and Disease. Science 279, 514-519 (1998).

[2] Alberts, B. et al. Molecular Biology of the Cell (Garland Science, 2008), 5. edn.

[3] Fletcher, D. A. \& Mullins, R. D. Cell mechanics and the cytoskeleton. Nature 463, 485-492 (2010).

[4] Tilney, L. G., Derosier, D. J. \& Mulroy, M. J. The organization of actin filaments in the stereocilia of cochlear hair cells. J. Cell Biol. 86, 244-259 (1980).

[5] Fuchs, E. The Cytoskeleton and Disease: Genetic Disorders of Intermediate Filaments. Annu. Rev. Genet. 30, 197-231 (1996).

[6] Irvine, A. D. \& Mclean, W. H. I. Human keratin diseases: the increasing spectrum of disease and subtlety of the phenotype-genotype correlation. Brit. J. Dermatol. 140, 815-828 (1999).

[7] Omary, M. B., Coulombe, P. A. \& McLean, W. I. Intermediate Filament Proteins and Their Associated Diseases. New Engl. J. Med. 351, 2087-2100 (2004).

[8] Paunesku, T., Vogt, S., Maser, J., Lai, B. \& Woloschak, G. X-ray fluorescence microprobe imaging in biology and medicine. J. Cell. Biochem. 99, 1489-1502 (2006).

[9] Shapiro, D. et al. Biological imaging by soft x-ray diffraction microscopy. Proc. Natl. Acad. Sci. USA 102, 15343-15346 (2005).

[10] Giewekemeyer, K. et al. Quantitative biological imaging by ptychographic xray diffraction microscopy. Proc. Natl. Acad. Sci. USA 107, 529-534 (2010).

[11] Jiang, H. et al. Quantitative 3D imaging of whole, unstained cells by using X-ray diffraction microscopy. Proc. Natl. Acad. Sci. USA 107, 11234-11239 $(2010)$. 
[12] Wilke, R. N. et al. Hard X-ray imaging of bacterial cells: nano-diffraction and ptychographic reconstruction. Opt. Express 20, 19232-19254 (2012).

[13] Fratzl, P., Jakob, H. F., Rinnerthaler, S., Roschger, P. \& Klaushofer, K. Position-Resolved Small-Angle X-ray Scattering of Complex Biological Materials. J. Appl. Crystallogr. 30, 765-769 (1997).

[14] Lichtenegger, H., Müller, M., Paris, O., Riekel, C. \& Fratzl, P. Imaging of the helical arrangement of cellulose fibrils in wood by synchrotron X-ray microdiffraction. J. Appl. Crystallogr. 32, 1127-1133 (1999).

[15] Paris, O. From diffraction to imaging: New avenues in studying hierarchical biological tissues with x-ray microbeams (Review). Biointerphases 3, FB16FB26 (2008).

[16] Müller, B. et al. Scanning x-ray scattering: Evaluating the nanostructure of human tissues. Eur. J. Nanomed. 3, 30-33 (2010).

[17] Bunk, O. et al. Multimodal x-ray scatter imaging. New J. Phys. 11, 123016 (2009)

[18] Rinnerthaler, S. et al. Scanning small angle x-ray scattering analysis of human bone sections. Calcif. Tissue Int. 64, 422-429 (1999).

[19] Gourrier, A. et al. Scanning X-ray imaging with small-angle scattering contrast. J. Appl. Crystallogr. 40, s78-s82 (2007).

[20] Stangl, J., Mocuta, C., Diaz, A., Metzger, T. H. \& Bauer, G. X-Ray Diffraction as a Local Probe Tool. Chem. Phys. Chem. 10, 2923-2930 (2009).

[21] Kang, H. C. et al. Focusing of hard x-rays to 16 nanometers with a multilayer Laue lens. Appl. Phys. Lett. 92, 221114 (2008).

[22] Schropp, A. et al. Hard x-ray nanobeam characterization by coherent diffraction microscopy. Appl. Phys. Lett. 96, 091102 (2010).

[23] Mimura, H. et al. Breaking the $10 \mathrm{~nm}$ barrier in hard-X-ray focusing. Nat. Phys. 6, 122-125 (2010).

[24] Döring, F. et al. Sub-5 $\mathrm{nm}$ hard x-ray point focusing by a combined Kirkpatrick-Baez mirror and multilayer zone plate. Opt. Express 21, 1931119323 (2013). 
[25] Thibault, P., Elser, V., Jacobsen, C., Shapiro, D. \& Sayre, D. Reconstruction of a yeast cell from X-ray diffraction data. Acta Crystallogr. Sect. A 62, 248-261 (2006).

[26] Larabell, C. A. \& Nugent, K. A. Imaging cellular architecture with X-rays. Curr. Opin. Struct. Biol. 20, 623-631 (2010).

[27] Bartels, M. et al. Low-dose three-dimensional hard x-ray imaging of bacterial cells. Optical Nanoscopy 1, 10 (2012).

[28] Nam, D. et al. Imaging Fully Hydrated Whole Cells by Coherent X-Ray Diffraction Microscopy. Phys. Rev. Lett. 110, 098103 (2013).

[29] Koch, M. H., Vachette, P. \& Svergun, D. I. Small-angle scattering: a view on the properties, structures and structural changes of biological macromolecules in solution. Q. Rev. Biophys. 36, 147-227 (2003).

[30] Putnam, C. D., Hammel, M., Hura, G. L. \& Tainer, J. A. X-ray solution scattering (SAXS) combined with crystallography and computation: defining accurate macromolecular structures, conformations and assemblies in solution. Q. Rev. Biophys. 40, 191-285 (2007).

[31] Rambo, R. P. \& Tainer, J. A. Super-Resolution in Solution X-Ray Scattering and Its Applications to Structural Systems Biology. Annu. Rev. Biophys. 42, 415-441 (2013).

[32] Carter, E. A. et al. Silicon nitride as a versatile growth substrate for microspectroscopic imaging and mapping of individual cells. Mol. BioSyst. 6, 1316-1322 (2010).

[33] Sedlmair, J. et al. Imaging of Vascular Smooth Muscle Cells with Soft X-Ray Spectromicroscopy. Microsc. Microanal. 17, 991-1001 (2011).

[34] Dootz, R., Evans, H., Köster, S. \& Pfohl, T. Rapid Prototyping of X-Ray Microdiffraction Compatible Continuous Microflow Foils. Small 3, 96-100 (2007).

[35] Evans, H. M., Dootz, R., Köster, S., Struth, B. \& Pfohl, T. X-ray microdiffraction on flow-controlled biomolecular assemblies. Bulletin of the Polish Academy of Science - Technical Sciences 55, 217-227 (2007).

[36] Barrett, R. et al. X-ray microfocussing combined with microfluidics for on-chip X-ray scattering measurements. Lab Chip 6, 494-499 (2006). 
[37] Brennich, M. E. et al. Dynamics of intermediate filament assembly followed in micro-flow by small angle X-ray scattering. Lab Chip 11, 708-716 (2011).

[38] Köster, S. \& Pfohl, T. X-Ray Studies of Biological Matter in Microfluidic Environments. Mod. Phys. Lett. B 26, 1230018 (2012).

[39] Weinhausen, B. et al. X-ray nano-diffraction on cytoskeletal networks. New J. Phys. 14, 085013 (2012).

[40] Weinhausen, B. \& Köster, S. Microfluidic devices for X-ray studies on hydrated cells. Lab Chip 13, 212-215 (2013).

[41] Weinhausen, B. et al. Scanning X-ray Nano-Diffraction of Living Eukaryotic Cells in Microfluidic Environments. submitted to Phys. Rev. Lett. (2013).

[42] Herrmann, H., Strelkov, S. V., Burkhard, P. \& Aebi, U. Intermediate filaments: primary determinants of cell architecture and plasticity. J. Clin. Invest. 119, 1772-1783 (2009).

[43] Eriksson, J. E. et al. Introducing intermediate filaments: from discovery to disease. J. Clin. Invest. 119, 1763-1771 (2009).

[44] Toivola, D., Strnad, P., Habtezion, A. \& Omary, M. Intermediate filaments take the heat as stress proteins. Trends Cell Biol. 20, 79-91 (2010).

[45] Herrmann, H., Bar, H., Kreplak, L., Strelkov, S. V. \& Aebi, U. Intermediate filaments: from cell architecture to nanomechanics. Nat. Rev. Mol. Cell Biol. 8, 562-573 (2007).

[46] Herrmann, H., Wedig, T., Porter, R. M., Lane, E. B. \& Aebi, U. Characterization of Early Assembly Intermediates of Recombinant Human Keratins. J. Struct. Biol. 137, 82-96 (2002).

[47] Owens, D. W. \& Lane, E. B. The quest for the function of simple epithelial keratins. Bioessays 25, 748-758 (2003).

[48] Yamada, S., Wirtz, D. \& Coulombe, P. A. Pairwise assembly determines the intrinsic potential for self-organization and mechanical properties of keratin filaments. Mol. Biol. Cell 13, 382-391 (2002).

[49] Sun, T. T. \& Green, H. Keratin filaments of cultured human epidermal cells. Formation of intermolecular disulfide bonds during terminal differentiation. $J$. Biol. Chem. 253, 2053-2060 (1978). 
[50] Nelson, W. G. \& Sun, T.-T. The 50-and 58-kdalton keratin classes as molecular markers for stratified squamous epithelia: cell culture studies. J. Cell Biol. 97, 244-251 (1983).

[51] Bragulla, H. H. \& Homberger, D. G. Structure and functions of keratin proteins in simple, stratified, keratinized and cornified epithelia. J. Anat. 214, 516-559 (2009).

[52] Herrmann, H. \& Aebi, U. Intermediate Filaments: Molecular Structure, Assembly Mechanism, and Integration Into Functionally Distinct Intracellular Scaffolds. Annu. Rev. Biochem. 73, 749-789 (2004).

[53] Lee, C.-H., Kim, M.-S., Chung, B. M., Leahy, D. J. \& Coulombe, P. A. Structural basis for heteromeric assembly and perinuclear organization of keratin filaments. Nat. Struct. Mol. Biol. 19, 707-715 (2012).

[54] Lichtenstern, T., Mücke, N., Aebi, U., Mauermann, M. \& Herrmann, H. Complex formation and kinetics of filament assembly exhibited by the simple epithelial keratins K8 and K18. J. Struct. Biol. 177, 54-62 (2012).

[55] Ma, L., Yamada, S., Wirtz, D. \& Coulombe, P. A. A 'hot-spot' mutation alters the mechanical properties of keratin filament networks. Nat. Cell Biol. 3, 503-506 (2001).

[56] Lee, C.-H. \& Coulombe, P. A. Self-organization of keratin intermediate filaments into cross-linked networks. J. Cell Biol. 186, 409-421 (2009).

[57] Kayser, J., Grabmayr, H., Harasim, M., Herrmann, H. \& Bausch, A. R. Assembly kinetics determine the structure of keratin networks. Soft Matter $\mathbf{8}$, 8873-8879 (2012).

[58] Listwan, P. \& Rothnagel, J. A. Keratin Bundling Proteins. In Omary, M. B. \& Coulombe, P. A. (eds.) Intermediate Filament Cytoskeleton, vol. 78 of Methods in Cell Biology, 817-827 (Academic Press, 2004).

[59] Mack, J. W., Steven, A. C. \& Steinert, P. M. The mechanism of interaction of filaggrin with intermediate filaments: the ionic zipper hypothesis. J. Mol. Biol. 232, 50-66 (1993).

[60] Bousquet, O. et al. The nonhelical tail domain of keratin 14 promotes filament bundling and enhances the mechanical properties of keratin intermediate filaments in vitro. J. Cell Biol. 155, 747-754 (2001). 
[61] Windoffer, R. \& Leube, R. E. De novo formation of cytokeratin filament networks originates from the cell cortex in A-431 cells. Cell Motil. Cytoskeleton 50, 33-44 (2001).

[62] Windoffer, R., Wöll, S., Strnad, P. \& Leube, R. E. Identification of Novel Principles of Keratin Filament Network Turnover in Living Cells. Mol. Biol. Cell 15, 2436-2448 (2004).

[63] Wöll, S., Windoffer, R. \& Leube, R. E. Dissection of keratin dynamics: different contributions of the actin and microtubule systems. Eur. J. Cell Biol. 84, 311-328 (2005).

[64] Windoffer, R., Kölsch, A., Wöll, S. \& Leube, R. E. Focal adhesions are hotspots for keratin filament precursor formation. J. Cell Biol. 173, 341-348 (2006).

[65] Kölsch, A., Windoffer, R. \& Leube, R. E. Actin-dependent dynamics of keratin filament precursors. Cell Motil. Cytoskeleton 66, 976-985 (2009).

[66] Kölsch, A., Windoffer, R., Würflinger, T., Aach, T. \& Leube, R. E. The keratin-filament cycle of assembly and disassembly. J. Cell Sci. 123, 22662272 (2010).

[67] Windoffer, R., Beil, M., Magin, T. M. \& Leube, R. E. Cytoskeleton in motion: the dynamics of keratin intermediate filaments in epithelia. J. Cell Biol. 194, 669-678 (2011).

[68] Strnad, P., Windoffer, R. \& Leube, R. E. Induction of rapid and reversible cytokeratin filament network remodeling by inhibition of tyrosine phosphatases. J. Cell Sci. 115, 4133-4148 (2002).

[69] Beil, M. et al. Sphingosylphosphorylcholine regulates keratin network architecture and visco-elastic properties of human cancer cells. Nat. Cell Biol. 5, 803-811 (2003).

[70] Beil, M., Braxmeier, H., Fleischer, F., Schmidt, V. \& Walther, P. Quantitative analysis of keratin filament networks in scanning electron microscopy images of cancer cells. J. Microsc. 220, 84-95 (2005).

[71] Flitney, E. W., Kuczmarski, E. R., Adam, S. A. \& Goldman, R. D. Insights into the mechanical properties of epithelial cells: the effects of shear stress on the assembly and remodeling of keratin intermediate filaments. The FASEB Journal 23, 2110-2119 (2009). 
[72] Gu, L.-H. \& Coulombe, P. A. Keratin function in skin epithelia: a broadening palette with surprising shades. Curr. Opin. Cell Biol. 19, 13-23 (2007).

[73] Sivaramakrishnan, S., DeGiulio, J. V., Lorand, L., Goldman, R. D. \& Ridge, K. M. Micromechanical properties of keratin intermediate filament networks. Proc. Natl. Acad. Sci. USA 105, 889-894 (2008).

[74] Sivaramakrishnan, S., Schneider, J. L., Sitikov, A., Goldman, R. D. \& Ridge, K. M. Shear Stress Induced Reorganization of the Keratin Intermediate Filament Network Requires Phosphorylation by Protein Kinase C $\zeta$. Mol. Biol. Cell 20, 2755-2765 (2009).

[75] Norlén, L. \& Al-Amoudi, A. Stratum Corneum Keratin Structure, Function, and Formation: The Cubic Rod-Packing and Membrane Templating Model. J. Investig. Dermatol. 123, 715-732 (2004).

[76] Norlén, L. Stratum corneum keratin structure, function and formation - a comprehensive review. Int. J. Cosmetic Sci. 28, 397-425 (2006).

[77] Fraser, R. D. B., MacRae, T. P. \& Rogers, G. E. Keratins. Their Composition, Structure and Biosynthesis (Charles C Thomas, 1972).

[78] Crick, F. H. C. Is $\alpha$-Keratin a Coiled Coil? Nature 170, 882-883 (1952).

[79] Spinelli, K. J. \& Gillespie, P. G. Bottoms up: transduction channels at tip link bases. Nat. Neurosci. 12, 529-530 (2009).

[80] LeMasurier, M. \& Gillespie, P. G. Hair-cell mechanotransduction and cochlear amplification. Neuron 48, 403-415 (2005).

[81] Mooseker, M. S. \& Tilney, L. G. Organization of an actin filament-membrane complex. Filament polarity and membrane attachment in the microvilli of intestinal epithelial cells. J. Cell Biol. 67, 725-743 (1975).

[82] Tojkander, S., Gateva, G. \& Lappalainen, P. Actin stress fibers-assembly, dynamics and biological roles. J. Cell Sci. 125, 1855-1864 (2012).

[83] Perrot, R., Berges, R., Bocquet, A. \& Eyer, J. Review of the multiple aspects of neurofilament functions, and their possible contribution to neurodegeneration. Mol. Neurobiol. 38, 27-65 (2008).

[84] Rao, M. V. et al. The neurofilament middle molecular mass subunit carboxylterminal tail domains is essential for the radial growth and cytoskeletal architecture of axons but not for regulating neurofilament transport rate. J. Cell Biol. 163, 1021-1031 (2003). 
[85] Pijak, D. S. et al. Neurofilament spacing, phosphorylation, and axon diameter in regenerating and uninjured lamprey axons. J. Comp. Neurol. 368, 569-581 (1996).

[86] Als-Nielsen, J. \& McMorrow, D. Elements of Modern X-Ray Physics (Wiley, 2011), 2. edn.

[87] Warren, B. E. X-Ray Diffraction (Dover, 1990).

[88] Paganin, D. M. Coherent X-ray Optics (Oxford University Press, 2006).

[89] Pfeiffer, F., Kottler, C., Bunk, O. \& David, C. Hard x-ray phase tomography with low-brilliance sources. Phys. Rev. Lett. 98, 108105 (2007).

[90] de Jonge, M. D. et al. Quantitative Phase Imaging with a Scanning Transmission X-Ray Microscope. Phys. Rev. Lett. 100, 163902 (2008).

[91] Thibault, P. et al. Contrast mechanisms in scanning transmission x-ray microscopy. Phys. Rev. A 80, 043813 (2009).

[92] Kaulich, B., Thibault, P., Gianoncelli, A. \& Kiskinova, M. Transmission and emission X-ray microscopy: operation modes, contrast mechanisms and applications. J. Phys.: Condens. Matter 23, 083002 (2011).

[93] Holt, M., Harder, R., Winarski, R. \& Rose, V. Nanoscale Hard X-Ray Microscopy Methods for Materials Studies. Annu. Rev. Mater. Res. 43, 183-211 (2013).

[94] Morrison, G. R. \& Browne, M. T. Dark-field imaging with the scanning transmission x-ray microscope. Rev. Sci. Instrum. 63, 611-614 (1992).

[95] Thibault, P. et al. High-Resolution Scanning X-ray Diffraction Microscopy. Science 321, 379-382 (2008).

[96] Dubslaff, M. et al. Scanning x-ray nanodiffraction: from the experimental approach towards spatially resolved scattering simulations. Nanoscale Res. Lett. 7, 553 (2012).

[97] Garman, E. F. \& Owen, R. L. Cryocooling and radiation damage in macromolecular crystallography. Acta Cryst. D 62, 32-47 (2005).

[98] Kmetko, J., Husseini, N. S., Naides, M., Kalinin, Y. \& Thorne, R. E. Quantifying X-ray radiation damage in protein crystals at cryogenic temperatures. Acta Cryst. D 62, 1030-1038 (2006). 
[99] Garman, E. F. Radiation damage in macromolecular crystallography: what is it and why should we care? Acta Cryst. D 66, 339-351 (2010).

[100] Ravelli, R. B. \& McSweeney, S. M. The 'fingerprint' that X-rays can leave on structures. Structure 8, 315-328 (2000).

[101] Holton, J. M. A beginner's guide to radiation damage. J. Synchrotron Rad. 16, 133-142 (2009).

[102] Howells, M. et al. An assessment of the resolution limitation due to radiationdamage in x-ray diffraction microscopy. J. Electron Spectrosc. Relat. Phenom. 170, 4-12 (2009).

[103] Teng, T.-y. \& Moffat, K. Primary radiation damage of protein crystals by an intense synchrotron X-ray beam. J. Synchrotron Rad. 7, 313-317 (2000).

[104] Riley, P. Free radicals in biology: oxidative stress and the effects of ionizing radiation. Int. J. Radiat. Biol. 65, 27-33 (1994).

[105] Roots, R. \& Okada, S. Estimation of life times and diffusion distances of radicals involved in X-ray-induced DNA strand breaks or killing of mammalian cells. Radiat. Res. 64, 306-320 (1975).

[106] Ward, J. F. Some biochemical consequences of the spatial distribution of ionizing radiation-produced free radicals. Radiat. Res. 86, 185-195 (1981).

[107] Iliakis, G. The role of DNA double strand breaks in lonizing radiation-induced killing of eukaryotic cells. Bioessays 13, 641-648 (1991).

[108] Sinclair, W. K. \& Morton, R. A. X-ray sensitivity during the cell generation cycle of cultured Chinese hamster cells. Radiat. Res. 29, 450-474 (1966).

[109] Chapman, J. D., Reuvers, A. P., Borsa, J. \& Greenstock, C. L. Chemical radioprotection and radiosensitization of mammalian cells growing in vitro. Radiat. Res. 56, 291-306 (1973).

[110] Roots, R., Chatterjee, A., Chang, P., Lommel, L. \& Blakely, E. Characterization of hydroxyl radical-induced damage after sparsely and densely ionizing irradiation. Int. J. Radiat. Biol. 47, 157-166 (1985).

[111] Nakano, H. \& Shinohara, K. X-ray-induced cell death: apoptosis and necrosis. Radiat. Res. 140, 1-9 (1994). 
[112] Belyakov, O. V., Prise, K. M., Trott, K. R. \& Michael, B. D. Delayed lethality, apoptosis and micronucleus formation in human fibroblasts irradiated with Xrays or alpha-particles. Int. J. Radiat. Biol. 75, 985-993 (1999).

[113] Schettino, G. et al. Low-dose studies of bystander cell killing with targeted soft X rays. Radiat. Res. 160, 505-511 (2003).

[114] Jones, J. G. A., Mills, C. N., Mogensen, M. A. \& Lee, C. I. Radiation dose from medical imaging: A primer for emergency physicians. West. J. Emerg. Med. 13, 202-210 (2012).

[115] Schroer, C. G. et al. Nanofocusing parabolic refractive x-ray lenses. Appl. Phys. Lett. 82, 1485-1487 (2003).

[116] Schroer, C. G. et al. Hard x-ray nanoprobe based on refractive x-ray lenses. Appl. Phys. Lett. 87, 124103 (2005).

[117] Patommel, J. Hard X-Ray Scanning Microscope Using Nanofocusing Parabolic Refractive Lenses. Ph.D. thesis, Technische Universität Dresden (2010).

[118] Kalbfleisch, S. et al. The holography endstation of beamline P10 at PETRA III. In AIP Conf. Proc., vol. 1234, 433 (2010).

[119] Kalbfleisch, S. et al. The Göttingen Holography Endstation of Beamline P10 at PETRA III/DESY. AIP Conf. Proc. 1365, 96-99 (2011).

[120] Salditt, T. et al. Partially coherent nano-focused x-ray radiation characterized by Talbot interferometry. Opt. Express 19, 9656-9675 (2011).

[121] Kalbfleisch, S. A Dedicated Endstation for Waveguide-based X-ray Imaging. Ph.D. thesis, Georg-August-Universität Göttingen (2012).

[122] Ice, G. E., Budai, J. D. \& Pang, J. W. L. The race to x-ray microbeam and nanobeam science. Science 334, 1234-1239 (2011).

[123] Snigirev, A. \& Snigireva, I. Hard X-ray Microoptics. In Modern Developments in X-Ray and Neutron Optics. Springer Series in Optical Sciences 137 (Springer, 2008).

[124] Takahashi, Y. et al. High-resolution and high-sensitivity phase-contrast imaging by focused hard x-ray ptychography with a spatial filter. Appl. Phys. Lett. 102, 094102 (2013). 
[125] Leibovitz, A., McCombs, W. B., Johnston, D., McCoy, C. E. \& Stinson, J. C. New human cancer cell culture lines. I. SW-13, small-cell carcinoma of the adrenal cortex. J. Natl. Cancer Inst. 51, 691-697 (1973).

[126] Stuhrmann, H. B. \& Miller, A. Small-angle scattering of biological structures. J. Appl. Crystallogr. 11, 325-345 (1978).

[127] Tivol, W. F., Briegel, A. \& Jensen, G. J. An Improved Cryogen for Plunge Freezing. Microsc. Microanal. 14, 375-379 (2008).

[128] Stöcker, H. (ed.) Taschenbuch der Physik (Wissenschaftlicher Verlag Harri Deutsch, 2005), 5. edn.

[129] Ryan, K. R. Cryofixation of tissues for electron microscopy: A review of plunge cooling methods. Scan. Microsc. 6, 715-743 (1992).

[130] Prahlad, V., Yoon, M., Moir, R. D., Vale, R. D. \& Goldman, R. D. Rapid Movements of Vimentin on Microtubule Tracks: Kinesin-dependent Assembly of Intermediate Filament Networks. J. Cell Biol. 143, 159-170 (1998).

[131] Xia, Y. \& Whitesides, G. M. Softlithographie. Angewandte Chemie 110, 568-594 (1998).

[132] McDonald, J. C. \& Whitesides, G. M. Poly(dimethylsiloxane) as a Material for Fabricating Microfluidic Devices. Acc. Chem. Res. 35, 491-499 (2002).

[133] Dhouib, K. et al. Microfluidic chips for the crystallization of biomacromolecules by counter-diffusion and on-chip crystal X-ray analysis. Lab Chip 9, 1412-1421 (2009).

[134] Decker, C. \& Jenkins, A. D. Kinetic approach of oxygen inhibition in ultraviolet- and laser-induced polymerizations. Macromolecules 18, 1241-1244 (1985).

[135] Stern, S. A., Shah, V. M. \& Hardy, B. J. Structure-permeability relationships in silicone polymers. J. Polym. Sci. B Polym. Phys. 25, 1263-1298 (1987).

[136] Menzel, A. et al. Scanning transmission X-ray microscopy with a fast framing pixel detector. Ultramicroscopy 110, 1143-1147 (2010).

[137] Krenkel, M., Bartels, M. \& Salditt, T. Transport of intensity phase reconstruction to solve the twin image problem in holographic x-ray imaging. Opt. Express 21, 2220-2235 (2013). 
[138] Dahl, R. \& Staehelin, L. A. High-pressure freezing for the preservation of biological structure: Theory and practice. J. Electron Micr. Tech. 13, 165174 (1989).

[139] Horridge, G. A. \& Tamm, S. L. Critical Point Drying for Scanning Electron Microscopic Study of Ciliary Motion. Science 163, 817-818 (1969).

[140] Deyhle, H., Bunk, O. \& Müller, B. Nanostructure of healthy and cariesaffected human teeth. Nanomed.: Nanotechnol. 7, 694-701 (2011).

[141] Gaiser, S., Deyhle, H., Bunk, O., White, S. \& Müller, B. Understanding Nano-Anatomy of Healthy and Carious Human Teeth: a Prerequisite for Nanodentistry. Biointerphases 7, 1-14 (2012). 10.1007/s13758-011-0004-8.

[142] Porod, G. Die Röntgenkleinwinkelstreuung von dichtgepackten kolloiden Systemen. Kolloid-Z. 124, 83-114 (1951). 10.1007/BF01512792.

[143] Glatter, O. \& Kratky, O. (eds.) Small Angle X-ray Scattering (Academic Press, 1982).

[144] Schmidt, P. W. et al. Small-angle x-ray scattering from the surfaces of reversed-phase silicas: Power-law scattering exponents of magnitudes greater than four. J. Chem. Phys. 94, 1474-1479 (1991).

[145] Lorenz-Haas, C. et al. Scanning Microfocus Small-Angle X-ray Scattering: A New Tool to Investigate Defects at Polymer-Polymer Interfaces. Langmuir 19, 3056-3061 (2003).

[146] Campo, M. G. \& Grigera, J. R. Classical molecular-dynamics simulation of the hydroxyl radical in water. J. Chem. Phys. 123, 084507 (2005).

[147] Kalwarczyk, T., Tabaka, M. \& Holyst, R. Biologistics-Diffusion coefficients for complete proteome of Escherichia coli. Bioinformatics 28, 2971-2978 (2012).

[148] Henke, B. L., Gullikson, E. M. \& Davis, J. C. X-ray interactions: photoabsorption, scattering, transmission, and reflection at $\mathrm{E}=50-30000 \mathrm{eV}, \mathrm{Z}=1-92$. At. Data Nucl. Data Tables 54, 181-342 (1993).

[149] Cole, A. Absorption of 20-eV to 50000-eV Electron Beams in Air and Plastic. Radiat. Res. 38, 7-33 (1969).

[150] Holz, M., Heil, S. R. \& Sacco, A. Temperature-dependent self-diffusion coefficients of water and six selected molecular liquids for calibration in accurate 1H NMR PFG measurements. Phys. Chem. Chem. Phys. 2, 4740-4742 (2000). 
[151] Lane, E. B., Goodman, S. L. \& Trejdosiewicz, L. K. Disruption of the keratin filament network during epithelial cell division. EMBO J. 1, 1365-1372 (1982).

[152] Kiernan, J. A. Formaldehyde, formalin, paraformaldehyde and glutaraldehyde: what they are and what they do. Microsc. Today 1, 8-12 (2000).

[153] Hell, S. W. \& Wichmann, J. Breaking the diffraction resolution limit by stimulated emission: stimulated-emission-depletion fluorescence microscopy. Opt. Lett. 19, 780-782 (1994).

[154] Meyer, L. et al. Dual-Color STED Microscopy at 30-nm Focal-Plane Resolution. Small 4, 1095-1100 (2008).

[155] Bale, H. D. \& Schmidt, P. W. Small-Angle X-Ray-Scattering Investigation of Submicroscopic Porosity with Fractal Properties. Phys. Rev. Lett. 53, 596-599 (1984).

[156] Teixeira, J. Experimental methods for studying fractal aggregates. In Stanley, H. E. \& Ostrowsky, N. (eds.) On Growth and From. Fractal and Non-Fractal Patterns in Physics, 145-162 (Martinus Nijhoff Publishers, 1986).

[157] Teixeira, J. Small-angle scattering by fractal systems. J. Appl. Cryst. 21, 781-785 (1988).

[158] Martin, J. E. \& Hurd, A. J. Scattering from fractals. J. Appl. Cryst. 20, 61-78 (1987).

[159] Schaefer, D. W. Polymers, fractals, and ceramic materials. Science 243, 1023-1027 (1989).

[160] Schmidt, P. W. Small-angle scattering studies of disordered, porous and fractal systems. J. Appl. Cryst. 24, 414-435 (1991).

[161] Roe, R.-J. Methods of X-Ray and Neutron Scattering in Polymer Science (Oxford University Press, 2000).

[162] Life Technologies Corporation. Phallotoxins. http://tools.invitrogen. com/content/sfs/manuals/mp00354.pdf, [Online; acess on 09/04/2013].

[163] Thibault, P., Dierolf, M., Bunk, O., Menzel, A. \& Pfeiffer, F. Probe retrieval in ptychographic coherent diffractive imaging. Ultramicroscopy 109, 338-343 (2009). 



\section{Publications}

- B. Weinhausen, O. Saldanha, R. N. Wilke, C. Dammann, M. Priebe, M. Burghammer, M. Sprung, and S. Köster. Scanning X-ray Nano-Diffraction of Living Eukaryotic Cells in Microfluidic Environments. submitted to Phys. Rev. Lett.

- S.-C. Gleber, B. Weinhausen, S. Köster, J. Ward, D. Vine, L. Finney, and S. Vogt. New Developments in Hard X-ray Fluorescence Microscopy for Insitu Investigations of Trace Element Distributions in Aqueous Systems of Soil Colloids. J. Phys. Conf. Ser. 463, 012005 (2013).

- B. Weinhausen, and S. Köster. Microfluidic devices for X-ray studies on hydrated cells. Lab Chip 13, 212-215 (2013).

- B. Weinhausen, J.-F. Nolting, C. Olendrowitz, J. Langfahl-Klabes, M. Reynolds, T. Salditt, and S. Köster. X-ray nano-diffraction on cytoskeletal networks. New J. Phys., 14, 085013 (2012).

- B. Weinhausen, S. Aeffner, T. Reusch, and T. Salditt. Acyl-Chain Correlation in Membrane Fusion Intermediates: X-Ray Diffraction from the Rhombohedral Lipid Phase. Biophys. J. 102, 2121-2129 (2012).

- S. Aeffner, T. Reusch, B. Weinhausen, and T. Salditt. Energetics of stalk intermediates in membrane fusion are controlled by lipid composition. Proc. Nat. Acad. Sci. USA 109, E1609-E1618 (2012).

- P. E. Schneggenburger, A. Beerlink, B. Weinhausen, T. Salditt, and U. Diederichsen. Peptide Model Helices in Lipid Membranes - Insertion, Positioning and Lipid Response on Aggregation Studied by X-ray Scattering Methods. Eur. Biophys. J. 40, 417-436 (2011).

- B. Brüning, M. C. Rheinstädter, A. Hiess, B. Weinhausen, T. Reusch, S. Aeffner, and T. Salditt. Influence of Cholesterol on the Collective Dynamics of the Phospholipid Acyl Chains in Model Membranes. Eur. Phys. J. E 31, 419-428 (2010). 
- S. Aeffner, T. Reusch, B. Weinhausen and T. Salditt. Membrane fusion intermediates and the effect of cholesterol: An in-house X-ray scattering study. Eur. Phys. J. E 30, 205-214 (2009). 


\section{Danksagung}

Die in dieser Arbeit vorgestellten Messungen und Ergebnisse wären ohne die Bemühung und freundliche Unterstützung vieler Personen nicht möglich gewesen. Bei ihnen möchte ich mich an dieser Stelle herzlich bedanken.

An erster Stelle möchte ich Prof. Dr. Sarah Köster für die Möglichkeit zur Anfertigung dieser Arbeit in ihrer Arbeitsgruppe und ihre umfassende Förderung danken. Die Diskussionen über die Ergebnisse meiner Arbeit oder über die weitere Vorgehensweise haben mich stets voran gebracht. Insbesondere möchte ich mich für die Ermöglichung meines Forschungsaufenthalts an der Advanced Photon Source bedanken.

Prof. Dr. Tim Salditt danke ich für die Übernahme des Koreferats dieser Arbeit und seine weitreichende Unterstützung wie auch für sein beständiges Interesse an meiner Arbeit. Die vielen Diskussionen der Ergebnisse haben mir immer neue Denkanstöße gegeben und unterschiedliche Blickwinkel für die Interpretation der Ergebnisse aufgezeigt. Für die Möglichkeit zur Teilnahme an Messzeiten seiner Arbeitsgruppe und die angenehme Arbeitsatmosphäre, möchte ich mich ebenfalls bedanken.

Prof. Dr. Helmut Grubmüller danke ich für sein stetiges Interesse an meiner Arbeit und für seine aktive Beteiligung in meinem Betreuungskommittee.

Prof. Dr. Simone Techert, Dr. Florian Rehfeldt und Prof. Dr. Andreas Janshoff danke ich für die Teilnahme an meinem Prüfungskommittee.

Dr. Ana Diaz and Dr. Andreas Menzel, I want to thank for their support during our measurements at the cSAXS beamline. In particular, I thank Ana for her ideas concerning the improvement of the set-up for our needs and her efforts to implement these.

Dr. Michael Reynolds, Dr. Emanuela DiCola, and Dr. Manfred Burghammer, I want to thank for their support during our measurements at the ID13 beamline. Particularly I thank Manfred for the helpful discussions and his continuous interest in our experiments.

Dr. Fabian Westermeier, Dr. Sergej Bondarenko, Dr. Alexey Zozulya, and Dr. 
Michael Sprung, I thank for their support of our beamtimes at the P10 beamline. Further, I thank Dr. Anja Burkhardt, Martin Warmer, and Dr. Alke Meents for the possibility to use the P11 Biology Laboratory for our sample preparations.

Dr. Sophie-Charlotte Gleber danke ich für den wunderbaren Aufenthalt an der Advanced Photon Source und ihre uneingeschränkte Gastfreundschaft. Der Aufenthalt war für mich sehr interessant und lehrreich und auch unsere ausgedehnten Radtouren bei jedem Wetter waren ein schönes Erlebnis. Außerdem danke ich Charlotte für die Einführung in die Präparation von Cryo-Proben.

Bei dem gesamten Institut für Röntgenphysik möchte ich mich für die sehr angenehme und freundschaftliche Arbeitsatmosphäre bedanken. Insbesondere Dr. Christian Olendrowitz, Christian Dammann, Jens Nolting, Oliva Saldanha und Dr. Valeria Piazza danke ich für die Unterstützung bei diversen Messzeiten. Ohne euch alle wären diese Messungen nicht möglich gewesen und die Messzeiten, besonders die Nachtschichten, wären nicht annährend so unterhaltsam gewesen. Marius Priebe, Marten Bernhardt, Robin Wilke, Dr. Matthias Bartels, Martin Krenkel, Dr. Sebastian Kalbfleisch, Bastian Hartmann und Dr. Markus Osterhoff danke ich für die Unterstützung bei unseren Messzeiten an der P10 Beamline. Insbesondere danke ich Robin für die kompetente Hilfestellung vor Ort und Markus für die schnellen Ferndiagnosen und Problembehebungen bei Softwareschwierigkeiten. Marius danke ich außerdem für die Einweisung in die Präparation von lyophilisierten Proben. Jannick Langfahl-Klabes danke ich für seine umfassende Einweisung in die Arbeit mit Zellkulturen und die Anfärbung von Zellen. Jan Goemann danke ich für seine schnelle Hilfe bei Computerproblemen. Joachim Herbst danke ich für seine Hilfe bei technischen Angelegenheiten und für das Lyophilisieren der Proben. Carsten Wulff und Peter Nieschalk danke ich für ihr unübertroffenes Engagement, welches es immer ermöglichte, aus vagen Ideen funktionierende Probenhalter oder andere Komponenten anzufertigen.

Für das Korrekturlesen dieser Arbeit danke ich Christian Dammann, Dr. Rita Graceffa, Clément Hémonnot, Jens Nolting, Rabea Sandmann und Robin Wilke.

Meinen Büromitbewohnern Dr. Julia Sedlmair, Dr. Mareike Mathes, Christian Dammann und Rabea Sandmann danke ich für die freundliche und hilfsbereite Arbeitsatmosphäre, in der sich auch sehr gute Freundschaften entwickeln konnten.

Meinen langjährigen Studienfreunden Steffi, Christian, Katha, Mira und Patrick danke ich für die schöne Zeit in und außerhalb der Uni. Patrizia und Pascal danke ich für die vielen unterhaltsamen Abende und gemeinsamen Unternehmungen. Corinna danke ich für die vielen gemeinsamen sportlichen Aktivitäten, die vor allem gegen Ende der Fertigstellung dieser Arbeit essentiell zum Kraftschöpfen beigetra- 
gen haben.

Ein ganz besonderer Dank gilt meinen Eltern Marianne und Uwe Weinhausen für ihre Unterstützung. Wochenendbesuche bei euch waren und sind immer ein Kurzurlaub für Leib und Seele.

Schließlich gilt Christoph mein besonders herzlicher Dank, für die grenzenlose Unterstützung, Aufmunterung, Motivation und Ablenkung. Vielen Dank, dass du wirklich immer verständnisvoll für mich da bist!

Für die finanzielle Unterstützung danke ich der Deutsche Telekom Stiftung, dem Dorothea Schlözer-Stipendienprogramm, der Göttinger Graduiertenschule für Neurowissenschaften, Biophysik und Molekulare Biowissenschaften und dem Sonderforschungsbereich 755 "Nanoscale Photonic Imaging". 



\section{Lebenslauf}

Britta Weinhausen

Geboren am 6. Mai 1986 in Seesen

Staatsangehörigkeit: Deutsch

\section{Schulischer und wissenschaftlicher Werdegang}

Seit 05/2010 Promotionsstudium der Physik an der Georg-AugustUniversität Göttingen

09/2011-10/2011 Forschungsaufenthalt an der Advanced Photon Source, Argonne, IL, USA

03/2010 Diplom in Physik, Titel der Diplomarbeit: "Strukturanalyse von Phospholipidmembranen in Abhängigkeit von der Hydratisation und dem Cholesteringehalt: Elektronendichteprofile und Ordnung der Acylketten"

10/2005-03/2010 Studium der Physik an der Georg-August-Universität Göttingen

06/2005 Erlangung der Allgemeinen Hochschulreife, JacobsonGymnasium Seesen 